(C) 2001 International Press

Adv. Theor. Math. Phys. 4 (2000) 995-1186

\title{
Triples, Fluxes, and Strings
}

\author{
Jan de Boer ${ }^{1}$, Robbert Dijkgraaf ${ }^{1,2}$, Kentaro Hori ${ }^{3}$, \\ Arjan Keurentjes ${ }^{4}$, John Morgan ${ }^{5}$, \\ David R. Morrison ${ }^{6}$, Savdeep Sethi ${ }^{7}$
}

${ }^{1}$ Institute for Theoretical Physics, University of Amsterdam, Valckenierstraat 65, 1018 XE Amsterdam, The Netherlands jdeboer@wins.uva.nl

${ }^{2}$ Institute for Theoretical Physics, University of Amsterdam, Valckenierstraat 65, 1018 XE Amsterdam, The Netherlands, and Korteweg-de Vries Institute for Mathematics, University of Amsterdam, Plantage Muidergracht 24, 1018 TV Amsterdam, The Netherlands rhd@wins.uva.nl

${ }^{3}$ Department of Physics, Harvard University, Cambridge, MA 02138, USA hori@infeld.harvard.edu

${ }^{4}$ LPTHE, Université Pierre et Marie Curie, Paris VI, Tour 16, 4 place Jussieu, F-75252 Paris Cedex 05, France, and Laboratoire de Physique Théorique de l'Ecole Normale Supérieure, 24 rue Lhomond, F-75231 Paris Cedex 05, France arjan@lpthe.jussieu.fr

${ }^{5}$ Department of Mathematics, Columbia University, New York, NY 10027, USA jm@math.columbia.edu

${ }^{6}$ Department of Mathematics, Duke University, Durham, NC 27708, USA drm@cgtp.duke.edu

${ }^{7}$ Enrico Fermi Institute, University of Chicago, Chicago, IL 60637, USA sethi@theory.uchicago.edu

e-print archive: http://xxx.lanl.gov/hep-th/0103170 


\begin{abstract}
We study string compactifications with sixteen supersymmetries. The moduli space for these compactifications becomes quite intricate in lower dimensions, partly because there are many different irreducible components. We focus primarily, but not exclusively, on compactifications to seven or more dimensions. These vacua can be realized in a number ways: the perturbative constructions we study include toroidal compactifications of the heterotic/type I strings, asymmetric orbifolds, and orientifolds. In addition, we describe less conventional $\mathrm{M}$ and $\mathrm{F}$ theory compactifications on smooth spaces. The last class of vacua considered are compactifications on singular spaces with non-trivial discrete fluxes.

We find a number of new components in the string moduli space. Contained in some of these components are $\mathrm{M}$ theory compactifications with novel kinds of "frozen" singularities. We are naturally led to conjecture the existence of new dualities relating spaces with different singular geometries and fluxes. As our study of these vacua unfolds, we also learn about additional topics including: $\mathrm{F}$ theory on spaces without section, automorphisms of del Pezzo surfaces, and novel physics (and puzzles) from equivariant K-theory. Lastly, we comment on how the data we gain about the $\mathrm{M}$ theory three-form might be interpreted.
\end{abstract}

\title{
Contents
}

1 Introduction and Summary

2.1 Gauge bundles on a torus . . . . . . . . . . 1009

2.1.1 Bundles on $S^{1} \ldots \ldots \ldots \ldots 10 \ldots$

$2.1 .2 \quad$ Bundles on $T^{2} \ldots \ldots \ldots \ldots 12$

2.1.3 Bundles on $T^{3} \ldots \ldots \ldots 10$. . . . . . . . . . 1013

2.1.4 Bundles on $T^{4} \ldots \ldots \ldots$. . . . . . . . . . 1017 
2.1.5 Bundles on $T^{5} \ldots \ldots \ldots \ldots 18 \ldots$

2.2 Anomaly cancellation . . . . . . . . . . . . . . 1019

2.2 .1 A perturbative argument . . . . . . . . . . . . 1019

2.2.2 An $\mathrm{M}$ theory argument . . . . . . . . . . . . 1020

2.3 Gauge bundles in string theory . . . . . . . . . . . . . . 1020

2.3.1 Holonomy in string theory I: Narain compactification 1021

2.3.2 The topology of subgroups in string theory . . . . . . 1024

2.3.3 Holonomy in string theory II: asymmetric orbifolds . . 1028

2.3.4 Triples in string theory $\ldots \ldots \ldots 30$

2.3.5 Anomaly cancellation and winding states . . . . . 1034

2.4 Moduli spaces . . . . . . . . . . . . . . . . . . 1036

2.4.1 Lattices for the orbifolds . . . . . . . . . . . . . 1036

2.4 .2 Dualities . . . . . . . . . . . . . . . . . . . . 1042

2.4.3 Degeneration limits: connections to other models . . . 1052

2.4.4 A strong coupling description of the $\mathbb{Z}_{2}$ triple $\ldots \ldots 1055$

\section{Orientifolds}

3.1 Background and definitions $\ldots \ldots \ldots \ldots 56$

3.1.1 The closed string perturbation expansion $\ldots \ldots$. . 1057

3.1 .2 Some pathologies . . . . . . . . . . . . . 1060

3.2 The classification . . . . . . . . . . . . . . . . 1061

$3.2 .1 \quad D=10 \ldots \ldots \ldots \ldots \ldots \ldots 1$ 


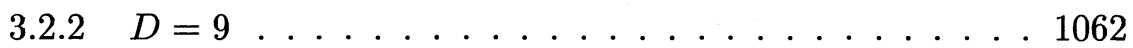

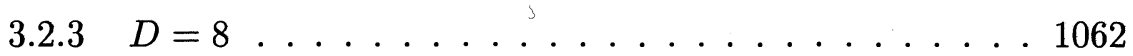

$3.2 .4 \quad D=7 \ldots \ldots \ldots \ldots \ldots \ldots \ldots \ldots \ldots$

$3.2 .5 \quad D=6 \ldots \ldots \ldots \ldots \ldots \ldots \ldots \ldots \ldots$

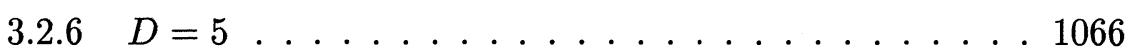

4 Compactifications of M and F Theory 1068

4.1 Some preliminary comments . . . . . . . . . . . . 1068

4.2 Six-dimensional $\mathrm{M}$ theory compactifications without fluxes . . 1072

$4.3 \quad \mathrm{~F}$ theory compactifications without flux . . . . . . 1076

4.3.1 From $\mathrm{F}$ theory to type $\mathrm{I}^{\prime} \ldots \ldots \ldots$. . . . . . . . 1084

4.3.2 Automorphisms of del Pezzo surfaces . . . . . . . . . 1086

4.4 Type IIA compactifications with RR one-form flux . . . . . 1093

4.4.1 Equivariant flat line bundles on $T^{4} \ldots \ldots \ldots$. . . 1093

4.4 .2 Local holonomies . . . . . . . . . . . . . . . . . . 1101

4.4.3 Singular K3 manifolds with one-form flux . . . . . . 1104

4.5 Fluxes and K-theory . . . . . . . . . . . . . . . 1107

4.5.1 K-theory description of RR fluxes . . . . . . . 1107

$4.5 .2 \quad$ K-theory on orbifolds $\ldots \ldots \ldots$. . . . . 1110

4.5.3 One-form fluxes in K-theory . . . . . . . . . . . . 1112

4.5.4 Three-form fluxes in K-theory . . . . . . . . . . . . . 1113

4.5.5 An alternate method of computation . . . . . . . 1119 
4.6 M theory compactifications with three-form flux . . . . 1120

4.6.1 Frozen singularities and new dualities . . . . . . . 1121

4.6.2 Three-form flux as equivariant cohomology $\ldots \ldots$. . 1129

4.6.3 Three-form holonomies . . . . . . . . . . . . . 1131

4.6.4 An M theory dual of the CHL string? . . . . . . . 1136

4.6.5 Some comments on type IIA versus M theory . . . . 1139

4.6.6 The geometry of the three-form . . . . . . . . . 1143

4.6.7 F theory compactifications with flux . . . . . . 1146

5 Acknowledgements

B.1 Duality on $S^{1} \ldots \ldots \ldots \ldots \ldots \ldots \ldots$

B.2 Duality on $T^{n} \ldots \ldots \ldots \ldots \ldots \ldots \ldots \ldots \ldots$

$\begin{array}{ll}\text { C Classifying Orientifold Configurations } & 1153\end{array}$

C.1 Configurations on $T^{2} \ldots \ldots \ldots \ldots \ldots \ldots$

C.2 Configurations on $T^{3} \ldots \ldots \ldots \ldots \ldots 1156$

C.3 Configurations on $T^{4} \ldots \ldots \ldots \ldots \ldots \ldots$

C.4 Configurations on $T^{5} \ldots \ldots \ldots \ldots \ldots 1157$ 
D.1 Equivariant cohomology via spectral sequences . . . . . . 1159

D.2 The case of $T^{4} / G$ for more general $G \ldots \ldots \ldots \ldots$

D.3 Explicit generators for $H_{\mathbb{Z}_{2}}^{2}\left(T^{n}, \mathbb{Z}_{2}\right) \ldots \ldots \ldots \ldots \ldots \ldots$

\section{Introduction and Summary}

The moduli space of supersymmetric string compactifications is an immensely complicated object. One of the aspects that we might hope to understand are the discrete choices that characterize disconnected components of the moduli space. We shall focus on string compactifications with sixteen supersymmetries. Familiar examples of such compactifications are the heterotic string on a torus and $\mathrm{M}$ theory on a $K 3$ surface. With this much supersymmetry, the moduli space cannot be lifted by space-time superpotentials. The number of distinct components in the string moduli space can, however, change as we compactify to lower dimensions. For example, when compactified on a circle, there is a new component in the moduli space of the heterotic string which contains the CHL string $[1,2]$.

We shall primarily, but not exclusively, focus on compactifications to 7 or more dimensions. Our goal is to describe the different components of the string moduli space in each dimension. We also describe the various dual ways in which a given space-time theory can be realized in string theory or in $\mathrm{M}$ theory, its non-perturbative, mysterious completion. Down to 7 dimensions, it seems quite likely that our study of the string moduli space captures all the distinct components. However, a proof must await a deeper understanding of $\mathrm{M}$ theory. As we analyze the various components of the string moduli space, we will learn about new phenomena in string theory and some interesting mathematical relations. Many of the results we describe call for a deeper analysis, or suggest natural paths for further study. There 
are also tantalizing hints of how we might correctly treat the $\mathrm{M}$ theory 3form. Some of these hints suggest relations between the 3 -form and $E_{8}$ gauge bundles which are somewhat reminiscent of $[3,4]$. In the remainder of the introduction, we shall outline our results.

In the following section, we begin by describing the classification of flat bundles on a torus. While this might seem trivial at first sight, there are actually interesting new components in the moduli space of flat connections on $T^{3}$, and on higher-dimensional tori. On $T^{3}$, these new components correspond to "triples" of commuting flat connections which are not connected to the trivial connection - the case with no Wilson line. If we pick a connection in a given component of the moduli space, we can compute its Chern-Simons invariant. This is constant over a given component of the moduli space and actually uniquely characterizes each component of the moduli space. These new components in the gauge theory moduli space are the basis for a new set of components in the moduli space of the heterotic string on $T^{3}$. Our discussion of these heterotic/type I toroidal compactifications begins with a discussion of anomaly cancellation conditions, and continues with a study of asymmetric orbifold realizations together with the structure of the moduli space for these new components.

Let us summarize our findings: in 9 dimensions, we find only the 2 known components in the heterotic/type I string moduli space. The "standard" component unifies the conventional $E_{8} \times E_{8}$ string together with the $\operatorname{Spin}(32) / \mathbb{Z}_{2}$ heterotic/type I string. It is important for us to note that the gauge group for the $E_{8} \times E_{8}$ string is actually $\left(E_{8} \times E_{8}\right) \rtimes \mathbb{Z}_{2}$, as explained in section 2.1.1. ${ }^{1}$ The other component contains the CHL string. In 8 dimensions, we still have the standard component. There is still only one other component which now contains both the CHL string and the compactification of the type I string with no vector structure. The interesting new physics appears in 7 dimensions where we find 6 components in the

\footnotetext{
${ }^{1}$ Nevertheless, throughout this paper we use the common nomenclature, " $E_{8} \times E_{8}$ string."
} 
moduli space. These components can be labeled by a cyclic group $\mathbb{Z}_{m}$ for $m=1,2,3,4,5,6$. This cyclic group appears naturally in the construction of the $\mathbb{Z}_{m}$-triple for an $E_{8}$ gauge bundle. Like the CHL string, these new components have reduced rank and interesting space-time gauge groups like $F_{4}$ and $G_{2}$. The $m=1$ case is just the standard compactification of the heterotic string, while the $\mathbb{Z}_{2}$-triple contains the CHL string in its moduli space.

We then proceed in section 2.4.2 to describe duality chains relating heterotic compactifications with different gauge bundles. These chains generalize the usual T-duality relating the $\operatorname{Spin}(32) / \mathbb{Z}_{2}$ and $E_{8} \times E_{8}$ string on $S^{1}$. In some cases, we follow these chains all the way down to 5 dimensions finding new relations as we descend. The relations involve "quadruples" and "quintuples" which are analogues of triples for $T^{4}$ and $T^{5}$. Unfortunately, the classification of gauge bundles on tori of dimension greater than 3 is unknown. This is an outstanding open question. In section 2.4.4, we conclude our discussion of the heterotic string by describing an intriguing connection between the $\mathbb{Z}_{2}$-triple and a Horava-Witten style construction of the $E_{8} \times E_{8}$ string with background 3-form flux.

In section 3, we turn to orientifold string vacua. Proceeding again dimension by dimension, we find only the two previously known components in 9 dimensions: the first is the standard component containing the type I string. The second is the $(+,-)$ orientifold which contains no D-branes and has no enhanced gauge symmetry. We use + to refer to an $O^{+}$plane, - to refer to an $O^{-}$plane, and $-^{\prime}$ to refer to an $O^{-}$plane with a single stuck Dbrane. This notation and our conventions are explained more fully in section 3. This is a new component in the string moduli space beyond those with a dual heterotic description. In 8 dimensions, we find three components: the standard one, the orientifold realization of type I with no vector structure and the compactification of the $(+,-)$ orientifold which is the $(+,+,-,-)$ orientifold. Again, there is only one new component beyond those already described. 
In 7 dimensions, we again find new physics. The compactifications of the 8-dimensional constructions give three components. However, there is now an interesting subtlety with the case of $\left(+^{4},-{ }^{4}\right)$. We can imagine arranging the 8 orientifold planes on the vertices of a cube. However, there are 2 distinct ways of arranging the orientifold planes which are not diffeomorphic. The first arrangement is the one obtained by compactifying $(+,-)$ on $T^{2}$. The four + planes lie on a single face of the cube. The - planes lie on the four vertices of the opposite face. If we exchange one adjacent pair of + and - planes, we find an inequivalent configuration. As perturbative string compactifications, we show that these two configurations are inequivalent. ${ }^{2}$ Whether these orientifolds are distinct non-perturbatively is more subtle to determine, and we comment on this in section 4.6.1. This question of how we order the orientifold planes continues to be important in lower-dimensional compactifications. Therefore, there are two new components in the moduli space of perturbative string compactifications to 7 dimensions. We also give evidence against the existence of an $\mathrm{O6}^{\mathrm{-}^{\prime}}$ plane- $\mathrm{a}$ conclusion arrived at independently using different arguments in [6].

In dimensions below 7, our classification of orientifold configurations is no longer complete. However, we find evidence for a number of interesting relations including a 6 -dimensional duality between $\left(+^{\prime 4},-^{\prime 12}\right)$ and a quadruple compactification of type I with no vector structure. We also find evidence for a 5 -dimensional equivalence between $\left(-{ }^{32}\right)$ and $\left(+{ }^{16},-{ }^{16}\right)$. There are a host of open questions concerning the complete classification of orientifold configurations below 7 dimensions, the action of S-duality etc.

In section 4, we turn to $\mathrm{M}$ and $\mathrm{F}$ theory compactifications. Our starting point is 6-dimensional $\mathrm{M}$ theory compactifications without flux. The compactifications we study are on spaces of the form $\left(Z \times S^{1}\right) / G$ where $Z=K 3$ or $T^{4}$ and $G$ is a discrete group acting freely. For $Z=K 3$, the choice of groups $G$ has been classified by Nikulin. In our M theory context, the possible choices are $G=\mathbb{Z}_{m}$ with $m=1, \ldots, 8$, while for $Z=T^{4}, G=\mathbb{Z}_{n}$ with

\footnotetext{
${ }^{2}$ An interesting paper with a similar conclusion appeared shortly after our paper [5].
} 
$n=2,3,4,6$. We describe both the lattices for these compactifications and the singularities of $Z / G$. Only some of these $\mathrm{M}$ theory compactifications can be lifted to 7-dimensional $\mathrm{F}$ theory compactifications. For $Z=K 3$, the cases $m=1, \ldots, 6$ lift to new 7-dimensional theories which are dual descriptions of the heterotic triples constructed in section 2. It seems worth mentioning that studying D3-brane probes on these backgrounds, along the lines of [7-12], should be interesting.

All of the $Z=T^{4}$ theories lift to 7 dimensions. The case $G=\mathbb{Z}_{2}$ is another description of the compactified $(+,-)$ orientifold while the 3 remaining cases are new components in the string theory moduli space. We also point out the existence of a new $\mathrm{F}$ theory vacuum in 6 dimensions associated with $G=\mathbb{Z}_{2} \times \mathbb{Z}_{2}$. In studying these vacua and their dual realizations, we arrive at a natural interpretation of $\mathrm{F}$ theory compactifications without section [13]: the type IIB circle which should decompactify under $M$ theory/F theory duality as the volume of the elliptic fiber on the M-theory side goes to zero has a non-trivial twist. On decompactifying the circle, the twist becomes irrelevant and we gain additional degrees of freedom beyond those that we might have expected. The $\mathrm{F}$ theory compactification then "attaches" to a larger moduli space.

We proceed in section 4.3.2 to study del Pezzo surfaces with automorphisms. We show that the list of possible automorphisms of del Pezzo surfaces is classified by exactly the same data that classifies commuting triples of $E_{8}$. This is naturally suggested by the existence of $F$ theory duals for the heterotic triples, and confirmed by direct analysis. This also suggests a possible way of classifying $E_{8}$ bundles on higher-dimensional tori using a purely geometric analysis. We also recover our heterotic anomaly matching condition directly from the geometric analysis.

Compactifications with flux are the next topic of discussion. In section 4.4 , we start by describing type IIA compactifications on quotient spaces $Z / G$ with RR 1-form flux. These arise by reducing M theory on $\left(Z \times S^{1}\right) / G$ 
to type IIA on the $S^{1}$ fiber. These models generalize the 6 -dimensional Schwarz-Sen model which is dual to the 6-dimensional CHL string [14]. We begin by describing equivariant line bundles on $T^{4}$ and the computation of the relevant equivariant cohomology groups. This approach is naturally suggested from our geometric $M$ theory starting point. We then proceed to explain in what sense the 1-form flux is actually localized at the singularities of $T^{4} / G$ by studying the local holonomies for these bundles. We then generalize our discussion to the case of singular $K 3$ surfaces. This gives us a technique for finding the group of 1-form fluxes given the sublattice of vanishing cycles of the singular $K 3$ surface.

The description of RR charges and fields in type II string theory seems to involve K-theory rather than cohomology, at least at zero string coupling. In section 4.5, we study torsion RR 1-form and 3-form fluxes on orbifolds from the perspective of equivariant $\mathrm{K}$-theory. Our analysis is for local singularities of the form $\mathbb{C}^{2} / G$. As usual, to preserve supersymmetry, $G$ should lead to singularities of $A D E$-type. A torsion 1-form RR flux can be measured by a D0-brane, while a 3-form RR flux can be measured by a D2-brane. In both cases, the D-brane acquires an additional phase factor in the string theory path-integral. We describe how this phase can be computed for a given flux in terms of a reduced eta-invariant for the virtual bundle representing the flux.

The group of RR 1-form fluxes (modulo higher fluxes in a sense explained in section 4.5) is given by $H^{1}(G, U(1))$ which agrees with the result from equivariant cohomology. This is reassuring since we expect to be able to trust a straightforward analysis of fluxes for type II backgrounds that descend from purely geometric $\mathrm{M}$ theory compactifications. The case of RR 3-form flux is more interesting: with vanishing 1-form flux, we find that the group of 3-form fluxes is given by $H^{3}(G, U(1))$. However, the full group of 1-form and 3-form fluxes exhibits an unusual additive structure. The physical interpretation of this effect is that 3-brane flux can be induced by the presence of 1-brane flux: the 3-brane flux has a shifted quantization law. It might be possible 
to verify this from a dual description, perhaps one involving branes along the lines of [15]. This is quite critical because our later results suggest that it is far from clear that equivariant $\mathrm{K}$-theory is the right framework even in string theory. For example, from equivariant K-theory, we find $\mathbb{Z}_{120}$ as the group of RR 3-form fluxes supported by an $E_{8}$ singularity. Are all of these fluxes actually possible, or are some choices inconsistent?

In section 4.5.5, we present an alternate algebraic method for computing the desired K-theory quotients. The groups arrived at via this method confirm the results obtained from the reduced eta-invariant approach.

In section 4.6, we turn to the issue of $\mathrm{M}$ theory compactifications with flux. We are immediately met by the challenge of not knowing the correct framework in which to study the $\mathrm{M}$ theory 3 -form. This is a basic problem for smooth compactifications. In our case, the problem is only compounded by the fact that our compactifications involve singular geometries. The only previously known case is that of a $D_{4+n}$ singularity which comes in two flavors: a conventional resolvable singularity with space-time gauge group $S O(8+2 n)$, and a partially frozen variety with gauge group $S p(n)[16,17]$. The $D_{4}$ frozen singularity appears in the $\mathrm{M}$ theory description of $\mathrm{O6}^{+}$planes. In section 4.6.1, we argue that our new 7-dimensional components in the string moduli space imply the existence of frozen variants of $E_{6}, E_{7}$ and $E_{8}$ singularities. Each of these singularities can support a variety of fluxes with different associated space-time gauge groups. For example, $E_{8}$ comes in 5 frozen, or partially frozen, variants. This result is starkly different from what we might expect, for example, from equivariant K-theory.

We propose $\mathrm{M}$ theory duals for our new 7-dimensional heterotic models, and for our new 7-dimensional $\mathrm{F}$ theory models. The $\mathrm{M}$ theory duals are on singular $K 3$ surfaces with various combinations of frozen $D$ and $E$ singularities. We then proceed to argue for the existence of dualities that map type IIA compactifications on singular spaces with RR 1-form flux to type IIA compactifications on spaces with completely different sets of singularities 
and RR 3-form flux.

In section 4.6.2, we turn to the possibility that 3-form flux could be described by equivariant cohomology - perhaps with additional consistency conditions from equations of motion, or anomalies. We describe the computation of the relevant equivariant cohomology group using $T^{4} / \mathbb{Z}_{2}$ as an example. Section 4.6.3 extends our discussion of 1-form holonomies to torsion 3-form fluxes. Working under the premise that the physical choices for 3-form flux form a subset of choices predicted by equivariant cohomology, we study the global orbifold $T^{4} / \widehat{\mathcal{D}}_{4}$ in section 4.6.4. This orbifold has 2 $D_{4}$ singularities, and we show that there is a choice of flux with holonomies localized at those singularities. This is a natural concrete proposal for the $\mathrm{M}$ theory dual of the 7-dimensional CHL string.

We turn to some puzzles in matching $M$ theory with type IIA in section 4.6.5. These puzzles involve the spectrum of 2-branes computed both in $M$ theory and type IIA. A generalization of the Freed-Witten anomaly [18] for D2-branes resolves the puzzle and leads us to speculate about a generalization of the anomaly in the context of K-theory. In section 4.6.6, we present some comments on the framework in which the $M$ theory 3-form should be studied. Using a line of reasoning suggested by anomalies in wrapped branes, we are actually able to reproduce our list of frozen singularities. This is quite exciting, although the arguments are preliminary, and leave many (interesting) unresolved questions. The final section concludes with a brief summary of known $\mathrm{F}$ theory compactifications with flux. We find no new models beyond those previously studied.

As a guide for the reader, we summarize our results on the moduli space of 7-dimensional string compactifications in table 1. This includes a listing of all (known) dual ways of realizing a given component of the moduli space. 


\begin{tabular}{|c|c|c|c|}
\hline $\begin{array}{c}\text { Heterotic } \\
\text { description }\end{array}$ & $\begin{array}{c}\text { Orientifold } \\
\text { description }\end{array}$ & $\begin{array}{c}\text { M theory on K3 } \\
\text { with frozen } \\
\text { singularities of type }\end{array}$ & $\begin{array}{c}\text { F theory } \\
\text { compactified on }\end{array}$ \\
\hline "standard component" & $\left(-^{8}\right)$ & smooth $K 3$ & $K 3 \times S^{1}$ \\
\hline $\begin{array}{c}\mathbb{Z}_{2} \text { triple } \\
\text { CHL string } \\
\text { no vector structure }\end{array}$ & $\left(-^{6},+^{2}\right)$ & $D_{4} \oplus D_{4}$ & $\left(K 3 \times S^{1}\right) / \mathbb{Z}_{2}$ \\
\hline $\mathbb{Z}_{3}$ triple & & $E_{6} \oplus E_{6}$ & $\left(K 3 \times S^{1}\right) / \mathbb{Z}_{3}$ \\
\hline $\mathbb{Z}_{4}$ triple & & $E_{7} \oplus E_{7}$ & $\left(K 3 \times S^{1}\right) / \mathbb{Z}_{4}$ \\
\hline $\mathbb{Z}_{5}$ triple & & $E_{8} \oplus E_{8}$ & $\left(K 3 \times S^{1}\right) / \mathbb{Z}_{5}$ \\
\hline $\mathbb{Z}_{6}$ triple & $\left(-{ }^{4},+^{4}\right)_{1}$ & $\left(D_{4}\right)^{4}$ & $\left(T^{4} \times S^{1}\right) / \mathbb{Z}_{2}$ \\
\hline & $\left(-{ }^{4},+^{4}\right)_{2}$ & & \\
\hline & & $\left(E_{6}\right)^{3}$ & $\left(T^{4} \times S^{1}\right) / \mathbb{Z}_{3}$ \\
\hline & & $D_{4} \oplus E_{7} \oplus E_{7}$ & $\left(T^{4} \times S^{1}\right) / \mathbb{Z}_{4}$ \\
\hline & & $D_{4} \oplus E_{6} \oplus E_{8}$ & $\left(T^{4} \times S^{1}\right) / \mathbb{Z}_{6}$ \\
\hline
\end{tabular}

Table 1: A summary of 7-dimensional string theories with 16 supercharges. 


\section{The Heterotic/Type I String on a Torus}

\subsection{Gauge bundles on a torus}

Let us begin by reviewing the choice of gauge bundles on tori. While we need specific results only for the case of an $E_{8}$ or $\operatorname{Spin}(32) / \mathbb{Z}_{2}$ bundle, we shall include some general comments independent of the choice gauge group. For a more detailed review of this topic as well as further references, see [19]. We want our gauge fields to have zero curvature. This ensures that when we turn to string theory, they contribute nothing to the energy. A flat connection of Yang-Mills theory with gauge group $G$ on $T^{n}$ is specified by a set of $n$ commuting elements of $G$, denoted $\Omega_{i}$. These Wilson lines, which specify the holonomies around the $n$ non-trivial cycles of the torus, are not unique. The same classical vacuum is also described by any other choice $\Omega_{i}^{\prime}$ obtained by a global gauge transformation,

$$
\Omega_{i}^{\prime}=g \Omega_{i} g^{-1}
$$

Classifying all flat connections on $T^{n}$ with gauge group $G$ therefore amounts to classifying all sets of commuting elements in $G$ up to simultaneous conjugation in $G$.

The simplest way to construct a set of commuting elements is as follows: exponentiating the Cartan subalgebra of $G$ gives a maximal torus $T_{G}$, which is an abelian subgroup of $G$. By choosing our $\Omega_{i} \in T_{G}$, we obtain a flat connection on $T^{n}$. For particular groups like $G=S U(N)$ or $G=S p(N)$, all flat connections are gauge equivalent to a flat connection with holonomies on a maximal torus, for any $n$. However, in general the moduli space of flat connections contains additional components beyond the one containing the trivial connection. This insight was crucial in resolving some puzzles about counting vacua in four-dimensional gauge theory [17, 20-25].

How do we describe the component of the moduli space containing the trivial connection? With all $n$ holonomies on a maximal torus, we can use 
a gauge transformation to set the corresponding gauge potentials $A_{i}$ to constant elements of the Cartan subalgebra. The centraliser of this connectionthe subgroup of $G$ commuting with each $\Omega_{i}$-clearly contains the maximal torus as a subgroup. Therefore the rank of the centraliser of this flat connection equals the rank of $G$. We can characterize elements of the Cartan subalgebra by vectors on the space $\mathbb{R}^{r}$ with $r$ the $\operatorname{rank}$ of $G$. To represent our $n$ holonomies, we can therefore choose $n$ vectors $\mathbf{a}_{i}$ where we identify vectors that differ by elements of the coroot lattice. This identification simply corresponds to quotienting out periodic gauge transformations. The resulting moduli space is then compact. Lastly, we can conjugate each $\Omega_{i}$ simultaneously by elements of the normalizer of $T_{G}$. This corresponds to further quotienting our moduli space by the action of the Weyl group $\mathcal{W}$ on each vector $\mathbf{a}_{i}$, simultaneously. In later applications to string theory, we shall deal exclusively with simply-laced groups where we can normalize the roots to have length $\sqrt{2}$. The roots and coroots can then be identified. As an example, let us take the familiar case of $S U(N)$ for which the moduli space is $\left(T^{N-1}\right)^{n} / \mathcal{W}$.

For other components of the moduli space, we typically have a reduction of the rank of the centraliser of a flat connection. It is clear in this case that we cannot simultaneously conjugate all holonomies into a maximal torus. However, it is possible to gauge transform to a set where each holonomy $\Omega_{i}$ can be written as the product of two commuting elements. One element is on a maximal torus while the second element implements a discrete transformation: either an outer automorphism, or a Weyl reflection. Let us now consider the possibilities for various choices of $n$.

\subsubsection{Bundles on $S^{1}$}

A flat connection on a circle is specified by a single holonomy $\Omega$. The topological types of bundles over $S^{1}$ are in natural one-to-one correspondence with $\pi_{0}(G)$. If $\Omega$ is in a component $G_{c}$ of $G$ connected to the identity, then 
we can always choose a maximal torus $T_{G}$ containing $\Omega$. The rank of the centraliser of $\Omega$ then equals the rank of $G$. To find something new, we require a component of $G$ not connected to the identity. It is clear that conjugation with $\Omega$ maps $G_{c}$ to itself. Therefore $\Omega$ represents an automorphism of $G_{c}$ and because $\Omega \notin G_{c}$, it is an outer automorphism. In order to realize a holonomy which acts as an outer automorphism of $G_{c}$, the gauge group $G$ must be disconnected. The gauge group $G$ typically takes the form $G=G_{c} \rtimes \Gamma$ where $\Gamma$ is a finite group (acting by outer automorphisms) and $\rtimes$ denotes semi-direct product.

The outer automorphisms of a compact, simple, connected, and simplyconnected Lie group are in correspondence with the symmetries of its Dynkin diagram. The only compact, connected, and simply-connected simple Lie groups with outer automorphisms are $S U(N), \operatorname{Spin}(2 N)$ and $E_{6}$. These outer automorphisms permute the nodes of the Dynkin diagram. Thus, gauge theories with gauge groups $S U(N) \rtimes \mathbb{Z}_{2}$ for $N>2, \operatorname{Spin}(8) \rtimes \mathfrak{S}_{3}$, $\operatorname{Spin}(2 N) \rtimes \mathbb{Z}_{2}$ for $N>4$, and $E_{6} \rtimes \mathbb{Z}_{2}$ all admit non-trivial bundles over $S^{1}$.

The abelian group $U(1)=S O(2)$ admits an outer automorphism. The group manifold $U(1)$ is a circle and the outer automorphism acts by reflection on the circle. It can be represented as complex conjugation on $U(1)$, or as an element of $O(2)$ with det $=-1$ when $G_{c}=S O(2)$. For the gauge group we take $G=U(1) \rtimes \mathbb{Z}_{2}=O(2)$.

A group $G$ with a subgroup containing multiple isomorphic factors gives another example. There are outer automorphisms which permute the isomorphic factors.

Turning to the cases of interest to us, we note that $\operatorname{Spin}(32) / \mathbb{Z}_{2}$ does not have an outer automorphism, although $\operatorname{Spin}(32)$ does. The group $\operatorname{Spin}(32)$ has two isomorphic spin representations that are interchanged by its outer automorphism. Only one of these spin representations occurs as a representation of $\operatorname{Spin}(32) / \mathbb{Z}_{2}$, and therefore the outer automorphism of $\operatorname{Spin}(32)$ 
does not descend to a symmetry of $\operatorname{Spin}(32) / \mathbb{Z}_{2}$.

Although $E_{8}$ itself does not admit any outer automorphisms, the product $E_{8} \times E_{8}$ has two isomorphic factors and therefore has an outer automorphism exchanging the two $E_{8}$ factors. Compactifying $\left(E_{8} \times E_{8}\right) \rtimes \mathbb{Z}_{2}$ gauge theory on a circle with holonomy interchanging the two $E_{8}$ factors leads to a theory that has rank reduced by 8 because the group elements invariant under the holonomy must be symmetric in the two $E_{8}$ factors. This construction is the one employed in [2] in their realisation of the 9-dimensional CHL theory [1].

\subsubsection{Bundles on $T^{2}$}

A flat connection on $T^{2}$ is specified by two commuting holonomies. Let us first dispense with some simple extensions of our prior discussion. We can always pick two holonomies, $\Omega_{i}$, on a maximal torus of $G$. A second possibility is to have an outer automorphism, as in our $S^{1}$ discussion, as one holonomy and a group element left invariant by this automorphism as a second holonomy.

The next question we should ask is whether gauge bundles can have any non-trivial topological types on $T^{2}$. The first obstruction is measured by an element of $H^{1}\left(T^{2} ; \pi_{0}(G)\right)$ as we discussed in section 2.1.1. Let us assume that $G$ is connected so this obstruction is trivial. A non-trivial topological type then requires a non-simply-connected group. For $E_{8}$, there are therefore no non-trivial choices. However, for $\operatorname{Spin}(32) / \mathbb{Z}_{2}$ there is a non-trivial choice. We begin with a general discussion about how this topological choice comes about. Take $G$ to be connected but pick a holonomy $\Omega_{1}$ for one cycle that has a disconnected centraliser $Z\left(\Omega_{1}\right)$. Elements of the disconnected part of $Z\left(\Omega_{1}\right)$ map the connected part to itself, and are allowed choices for the second holonomy $\Omega_{2}$. For simplicity, take $T^{2}=S^{1} \times S^{1}$. By dimensional reduction on the first circle with holonomy $\Omega_{1}$, we may regard this as a theory with gauge group $Z\left(\Omega_{1}\right)$ on the remaining $S^{1}$. Therefore this is to some extent the same as our previous example. This is the situation that 
occurs for 't Hooft's twisted boundary conditions [26].

The group $G$ should be non-abelian since we require a disconnected centraliser for $\Omega_{1} \in G$. Let us assume that $G$ is simple. A theorem by Bott, as quoted in [22,24], states that the centraliser of any element from a simple and simply-connected group is connected. Therefore $G$ should be a nonsimply-connected group. Examples of non-simply-connected groups include $S U(n), S p(n), S p i n(n), E_{6}$ and $E_{7}$ quotiented by a non-trivial subgroup $Z$ of their centers. Let us denote the simply-connected cover of $G$ by $\tilde{G}$. The allowed representations of the gauge group are then restricted to those which represent $Z$ by the identity element. We can now choose holonomies which commute in $G$ but commute to a non-trivial element in the kernel of $\tilde{G} \rightarrow G$ [27]. The obstruction for lifting $G$ bundles to $\tilde{G}$ bundles is measured by a characteristic class $\widetilde{w}_{2} \in H^{2}\left(T^{2}, Z_{\tilde{G}} / Z_{G}\right)$, where $Z_{\tilde{G}}$ and $Z_{G}$ are the centres of $\tilde{G}$ and $G$, respectively.

More explicitly for the case of $\operatorname{Spin}(32) / \mathbb{Z}_{2}$, there is one choice, measured by a generalized second Stiefel-Whitney class, which determines whether the compactification does or does not have "vector structure" $[17,28]$. The case of no vector structure corresponds to taking Wilson lines, $\left(\Omega_{1}, \Omega_{2}\right)$, which commute to the non-trivial element in the kernel of the map $\operatorname{Spin}(32) \rightarrow$ $\operatorname{Spin}(32) / \mathbb{Z}_{2}$. In the component without vector structure, the rank is reduced by 8 . This is our only discrete choice on $T^{2}$.

\subsubsection{Bundles on $T^{3}$}

The simplest way to construct commuting triples is to pick an element of the maximal torus that commutes with two holonomies constructed with the methods that we just described. Again, this is essentially a dimensional reduction of our prior discussion. However, we shall meet new possibilities on $T^{3}$.

For the groups $E_{8}$ and $\operatorname{Spin}(32) / \mathbb{Z}_{2}$, compactification on $T^{3}$ introduces 
no additional topological choice beyond the choice of the generalized StiefelWhitney class in $H^{2}\left(T^{3}, \mathbb{Z}_{2}\right)$. Up to automorphisms of $T^{3}$, there are two topological types for the case of $\operatorname{Spin}(32) / \mathbb{Z}_{2}$ : Bundles of trivial class which are liftable to $\operatorname{Spin}(32)$, and non-liftable bundles. If we choose coordinates $\left(x_{1}, x_{2}, x_{3}\right)$ for $T^{3}$, we can always choose these non-trivial bundles to be unliftable on the $T^{2}$ parametrized by $\left(x_{1}, x_{2}\right)$ and liftable on all other twotori. For $E_{8}$ bundles, there are no non-trivial topological choices.

Even after fixing this topological choice, there is the possibility of additional components in the moduli space of flat connections. For example, in the case with trivial generalized Stiefel-Whitney class, these additional components consist of connections with three holonomies, $\Omega_{i}$, which commute but which are not connected by a path of flat connections to the trivial connection.

Let us again begin by framing our discussion in more general terms, before turning to the special groups of interest to us. Let $G$ be simply connected. Pick an element $\Omega_{1}$ with centraliser $Z\left(\Omega_{1}\right)$ so that $Z\left(\Omega_{1}\right)$ contains a semisimple part $Z_{s s}\left(\Omega_{1}\right)$ that is not simply-connected. We can then choose holonomies $\Omega_{2}$ and $\Omega_{3}$ from $Z_{s s}\left(\Omega_{1}\right)$ that obey twisted boundary conditions: they commute in $Z_{s s}\left(\Omega_{1}\right)$ but their lifts $\tilde{\Omega}_{2}$ and $\tilde{\Omega}_{3}$ to the simply-connected cover $\tilde{Z}_{s s}\left(\Omega_{1}\right)$ do not commute. In this way, we achieve rank reduction even in a connected and simply-connected group. The groups $\operatorname{Spin}(n>7)$ and all exceptional groups have non-trivial triples of this kind [17,20-24].

If $G$ is not simply-connected (but still semisimple), it is also possible in specific cases to choose an element $\Omega_{1}$ with centraliser $Z\left(\Omega_{1}\right)$ such that the semisimple part $Z_{s s}\left(\Omega_{1}\right)$ has a fundamental group strictly larger than the fundamental group of $G$. Then we can pick elements $\Omega_{2}$ and $\Omega_{3}$ from $Z_{s s}\left(\Omega_{1}\right)$ so that their lifts $\tilde{\Omega}_{2}$ and $\tilde{\Omega}_{3}$ to the simply-connected cover $\tilde{Z}_{s s}\left(\Omega_{1}\right)$ commute up to an element that is not contained in the fundamental group of $G$. Compactifying with $\Omega_{1}, \Omega_{2}$ and $\Omega_{3}$ as holonomies leads to rank reduced theories, but the rank reduction can be larger than would be the case for 
a compactification with twisted boundary conditions on a two-torus and a third holonomy from the maximal torus.

The various possible flat connections are characterised by two sets of data: first, the topological type of the bundle measured by the generalized Stiefel-Whitney class. Second, for a fixed topological choice, there can be different components of the moduli space. An important characteristic of a connection $A$ in some component of the moduli space is its Chern-Simons invariant which is defined by,

$$
\int_{T^{3}} C S(A)=\frac{1}{16 \pi^{2} h} \int_{T^{3}} \operatorname{tr}\left(A d A+\frac{2}{3} A^{3}\right),
$$

where $h$ is the dual Coxeter number. The Chern-Simons $(C S)$ invariant is well-defined in $\mathbb{R} / \mathbb{Z}$ and is constant over a connected component of the moduli space. These invariants, which have been computed for all simple groups in [24], are typically rational for non-trivial components ${ }^{3}$. A key result is that these components can be distinguished by their $C S$ invariant [24]. In fact, fixing the generalized Stiefel-Whitney class, $C S$ embeds the set of components of the moduli space of a fixed type into $\mathbb{Q} / \mathbb{Z}$. By the order of a component $k$, we mean the order of its $C S$ invariant in $\mathbb{Q} / \mathbb{Z}$.

Tables 2 and 3 summarize the structure of the moduli space for $E_{8}$ and $\operatorname{Spin}(32) / \mathbb{Z}_{2}$. In the latter case, we include both bundles with and without vector structure. Note that there are 12 distinct components for $E_{8}$ and 6 for $\operatorname{Spin}(32) / \mathbb{Z}_{2}$. The Chern-Simons invariants of a component of order $k$ are of the form $n / k$ with $1 \leq n \leq k$ and $n$ relatively prime to $k$. There is exactly one component of order $k$ for each such $n$. For example in the $E_{8}$ case, there are 2 components with $k=6$. We can distinguish these two components by their $C S$ invariants which are $1 / 6$ and $5 / 6 \bmod \mathbb{Z}$, respectively. Denoting the moduli space of flat connections on $T^{3}$ of a given topological type by $\mathcal{M}$ and letting $X$ be a component of $\mathcal{M}$, we note that [24]

$$
\sum_{X \in \mathcal{M}}\left(\frac{1}{3} \operatorname{dim}(X)+1\right)=h .
$$

\footnotetext{
${ }^{3}$ Those that do not contain the trivial connection.
} 


\begin{tabular}{|c|c|c|c|}
\hline $\begin{array}{c}\text { Order of } \\
\text { Component }\end{array}$ & $\begin{array}{c}\text { Maximal Unbroken } \\
\text { Gauge Groups }\end{array}$ & Degeneracy & Dimension \\
\hline 1 & $E_{8}$ & 1 & 24 \\
2 & $F_{4}, C_{4}$ & 1 & 12 \\
3 & $G_{2}$ & 2 & 6 \\
4 & $A_{1}$ & 2 & 3 \\
5 & $\{e\}$ & 4 & 0 \\
6 & $\{e\}$ & 2 & 0 \\
\hline
\end{tabular}

Table 2: The structure of the moduli space for $E_{8}$.

\begin{tabular}{|c|c|c|c|c|}
\hline $\begin{array}{c}\text { Order of } \\
\text { Component }\end{array}$ & $\begin{array}{c}\text { Maximal Unbroken } \\
\text { Gauge Groups }\end{array}$ & Degeneracy & Dimension & $\begin{array}{c}\text { (No) Vector } \\
\text { Structure }\end{array}$ \\
\hline 1 & $D_{16}$ & 1 & 48 & VS \\
2 & $B_{12}$ & 1 & 36 & VS \\
2 & $D_{n} \times C_{m}, n+m=8$ & 2 & 24 & NVS \\
4 & $B_{n} \times C_{m}, n+m=5$ & 2 & 15 & NVS \\
\hline
\end{tabular}

Table 3: The structure of the moduli space for $\operatorname{Spin}(32) / \mathbb{Z}_{2}$.

Since there are no topological choices for $E_{8} \times E_{8}$, all solutions are characterized by the $C S$ invariant for each $E_{8}$ factor. The case of integer $C S$ invariant corresponds to no rank reduction. For $C S=\frac{1}{2}$, the rank is reduced by 4 , $C S=\left(\frac{1}{3}, \frac{2}{3}\right)$ give a rank reduction of $6, C S=\left(\frac{1}{4}, \frac{3}{4}\right)$ give a rank reduction of 7 , while $C S=\left(\frac{1}{5}, \frac{2}{5}, \frac{3}{5}, \frac{4}{5}, \frac{1}{6}, \frac{5}{6}\right)$ give a rank reduction of 8 . For $E_{8} \times E_{8}$ we therefore have 144 different combinations with possible rank reductions of $0,4,6,7,8,10,11,12,13,14,15$ and 16 . Note that for an $E_{8} \times E_{8}$ bundle where both factors have identical triples, we can further impose the CHL outer automorphism on one of the holonomies leading to rank reductions of $12,14,15$ and 16 . The moduli space of $\operatorname{Spin}(32) / \mathbb{Z}_{2}$ is slightly more intricate, but as we shall see in section 2.2 , we do not require a detailed description of the non-trivial components in the moduli space beyond the usual no vector structure compactification with $C S=0$. 


\subsubsection{Bundles on $T^{4}$}

As usual, we can extend our prior discussion to the case of $T^{4}$ in a simple way. We add a circle to $T^{3}$ and choose the holonomy around the circle to lie in the maximal torus of $G$, and commute with the other holonomies. However, there are again new possibilities that cannot be obtained this way. Beyond $T^{3}$, there is no complete analysis for the general case so we shall restrict ourselves to examples which naturally arise in string theory.

Let us begin our discussion with $\operatorname{Spin}(32)$. We note that the group $\operatorname{Spin}(32)$ admits holonomies that break the group to a subgroup of the form $\operatorname{Spin}(2 N) \times \operatorname{Spin}\left(2 N^{\prime}\right) \times G$, where $N, N^{\prime} \geq 4$ and $G$ is some product of $U(n)$-factors, possibly not semisimple, of rank $16-N-N^{\prime}$. In fact the semisimple part of this subgroup is two-fold connected so we should include a quotient by some $\mathbb{Z}_{2}$; however, we will ignore this subtlety since it plays no role in our current considerations. The point is that both $\operatorname{Spin}(2 N)$ and $\operatorname{Spin}\left(2 N^{\prime}\right)$ have non-trivial triples. We may therefore construct $\operatorname{Spin}(32)$ holonomies that implement a triple in each subgroup. This leads to a nontrivial quadruple. It results in a rank reduction of 8 which is twice the reduction of a triple. We also note that the $C S$ invariants defined for any sub-three-torus of $T^{4}$ are always integer.

A similar construction can be applied to the case of $\operatorname{Spin}(32) / \mathbb{Z}_{2}$ without vector structure. Pick a holonomy that breaks the group to a subgroup $\operatorname{Spin}(2 N) \times \operatorname{Spin}\left(2 N^{\prime}\right) \times G$, where $N, N^{\prime} \geq 6$ and $G$ is some (possibly not semisimple) group of rank $16-N-N^{\prime}$. We have again ignored the topology of the subgroup. We imposed the restriction $N, N^{\prime} \geq 6$ because $\operatorname{Spin}(2 N \geq 12)$ admits triples without vector structure that lead to rank reduction beyond the reduction that follows from no vector structure [24,29]. Constructing such a triple in each of the factors of $\operatorname{Spin}(2 N) \times \operatorname{Spin}\left(2 N^{\prime}\right)$ leads to two possibilities with rank reduction 14 . One of these has a half-integer $C S$ invariant on a three-torus, but the other has integer $C S$ on all sub-three-tori. We shall meet these gauge bundles when we discuss orientifold constructions 
in section 3 .

A new topological possibility that occurs in compactifiying $\operatorname{Spin}(32) / \mathbb{Z}_{2}$ without vector structure on $T^{4}$ was briefly described in [17]. Recall that the class $\widetilde{w}_{2}$ is an element of $H^{2}\left(T^{4}, \mathbb{Z}_{2}\right)=\mathbb{Z}_{2}^{6}$. The new possibility appears when $\widetilde{w}_{2}$, viewed as an antisymmetric $4 \times 4$ matrix, has maximal rank. This happens precisely when $\tilde{w}_{2}^{2} \neq 0$. Such bundles have no vector structure over two complementary two-tori. The orientifold realization of this type of bundle was discussed in [17].

Thus for $\operatorname{Spin}(32) / \mathbb{Z}_{2}$ bundles on $T^{4}$, we encounter altogether three new types of bundles. On the other hand, for $E_{8}$ or $E_{8} \times E_{8}$ no new bundles appear, since neither $E_{8}$ nor $E_{8} \times E_{8}$ admit non-trivial quadruples.

\subsubsection{Bundles on $T^{5}$}

A $T^{5}$ compactification can be achieved in the usual trivial way: add a circle to $T^{4}$ with a holonomy from the maximal torus chosen to commute with the other holonomies. For the groups of interest to us, there are some interesting new possibilities to which we now turn.

The group $\operatorname{Spin}(32)$ has an element whose centraliser is $(\operatorname{Spin}(16) \times$ $\operatorname{Spin}(16)) / \mathbb{Z}_{2}$. The group $\operatorname{Spin(16)}$ admits a non-trivial quadruple [22]. The argument proceeds along the lines sketched for $\operatorname{Spin}(32)$ : the group $\operatorname{Spin}(16)$ has elements that have as centraliser $(\operatorname{Spin}(8) \times \operatorname{Spin}(8)) / \mathbb{Z}_{2}$. Since Spin(8) is among the groups that have a non-trivial triple, we construct one in each factor of $\operatorname{Spin}(8) \times \operatorname{Spin}(8)$. This results in a non-trivial quadruple of $\operatorname{Spin}(16)$. Constructing a non-trivial quadruple in each factor of the $\operatorname{Spin}(16) \times \operatorname{Spin}(16) \supset \operatorname{Spin}(32)$ leads in turn to a non-trivial quintuple for $\operatorname{Spin}(32)$. For this case, the rank reduction is complete and equals 16.

On the other hand, the group $E_{8}$ has an element that has as its centraliser $\operatorname{Spin}(16) / \mathbb{Z}_{2}$. Constructing a non-trivial quadruple in $\operatorname{Spin}(16)$ leads to a non-trivial quintuple in $E_{8}$, with a complete rank reduction of 8 . For an 
$E_{8} \times E_{8}$ bundle on $T^{5}$, we can embed a quintuple in one or both $E_{8}$-factors. This leads to rank reductions of 8 and 16 , respectively.

\subsection{Anomaly cancellation}

\subsubsection{A perturbative argument}

Our primary interest is in constructing consistent string compactifications. We need to know which of the many possible gauge bundle configurations actually give anomaly-free compactifications. The issue can be addressed from multiple perspectives. Let us begin with the familiar heterotic/type I anomaly cancellation conditions. Let us phrase our discussion in the language of the heterotic string. Up to irrelevant coefficients, the NS-NS $H$-field of the heterotic string satisfies,

$$
H=d B+C S(\omega)-C S(A)
$$

where $\omega$ is the spin connection, and $A$ is the connection for either an $E_{8} \times E_{8}$ or $\operatorname{Spin}(32) / \mathbb{Z}_{2}$ bundle. For a toroidal compactification, $C S(\omega)$ vanishes. For a flat geometry to remain a solution of string theory, the $H$-field cannot have energy which would warp the background geometry. This requirement can only be satisfied if the $H$-field is torsion or trivial in cohomology. On $T^{3}$, there is no possibility for torsion so the $H$-field must be trivial. Integrating equation (2) over $T^{3}$ leads to the requirement that the total $C S$ invariant for $A$ vanish.

Anomaly cancellation therefore rules out all the components in table 3 with non-vanishing $C S$ invariant. We are left with two $\operatorname{Spin}(32) / \mathbb{Z}_{2}$ compactifications: the component of order one with vector structure, and the component of order two without vector structure but with vanishing $C S$ invariant. Of the 144 possible choices of $E_{8} \times E_{8}$ gauge bundle, only 12 survive. These choices correspond to taking two $E_{8}$ gauge bundles with opposite $C S$ invariants. 


\subsubsection{An $M$ theory argument}

For the case of the $E_{8} \times E_{8}$ string, we can revisit anomaly cancellation from the perspective of the strong coupling Horava-Witten description of $\mathrm{M}$ theory on $S^{1} / \mathbb{Z}_{2}$ [30]. Let us sketch the argument without worrying about overall constants that are not needed for this argument. In the presence of boundaries at $x^{11}=0$ and at $x^{11}=\pi$, the definition of the $\mathrm{M}$ theory four-form $G$ is modified. The component of $G$ with legs on the torus and a leg on $x^{11}$ satisfies [30],

$$
G_{11 x y z} \sim \delta\left(x^{11}\right) C S\left(A_{1}\right)+\delta\left(x^{11}-\pi\right) C S\left(A_{2}\right)+\ldots
$$

where $A_{1}$ and $A_{2}$ are connections for the $E_{8} \times E_{8}$ bundle. The terms omitted involve the $C$-field which is constant on $T^{3}$ (see section 2.4.4) . For a flat geometry like $T^{3}$, we require that $G / 2 \pi$ be an integral cohomology class [31]. Integrating eq. (3) over $T^{3} \times S^{1} / \mathbb{Z}_{2}$ then implies that the total $C S$ invariant must cancel between the two $E_{8}$ bundles. This is just the strong coupling version of perturbative anomaly cancellation.

\subsection{Gauge bundles in string theory}

We now turn to a detailed discussion of toroidal compactifications of the heterotic string. While our discussion is in the context of the weakly coupled string, supersymmetry should guarantee that results about moduli spaces remain uncorrected at strong coupling. Since the data specifying perturbative type I compactifications is identical to the data specifying $\operatorname{Spin}(32) / \mathbb{Z}_{2}$ heterotic compactifications, our results also apply to the type I string.

From our prior discussion, we saw that all gauge bundles on a torus can be characterised by commuting holonomies $\Omega_{i}$ which we can write in the form,

$$
\Omega_{i}=\exp \left(2 \pi i \mathbf{a}_{i}\right) \Theta_{i}
$$


where $\mathbf{a}_{i}$ an element of the Cartan subalgebra. The second factor $\Theta_{i}$ implements a discrete transformation (but may be set to the identity on the group). For the decomposition of the holonomy given in equation (4) to be unambiguous, we demand that the two factors commute with each other. The $\Theta_{i}$ implement automorphisms of the group lattice. These can be either inner automorphisms which constitute the Weyl group, or outer automorphisms. Let us denote the automorphism implemented by $\Theta_{i}$ by $\theta_{i}$, and note that our requirement of commutativity allows us to choose,

$$
\Theta_{i}^{-1} \exp \left(2 \pi i \mathbf{a}_{i}\right) \Theta_{i}=\exp \left(2 \pi i \theta_{i}\left(\mathbf{a}_{i}\right)\right) \Leftrightarrow \mathbf{a}_{i}=\theta_{i}\left(\mathbf{a}_{i}\right) .
$$

In string theory, this decomposition of the holonomy into a component on the maximal torus and a discrete part is convenient since the two factors are treated differently in the world-sheet conformal field theory.

The factor representing the maximal torus contribution can studied within the usual framework of Narain compactification [32,33]. The discrete factor $\Theta_{i}$ can be implemented by the asymmetric orbifold construction [34, 35]. Let us first turn to Narain compactifications, postponing the asymmetric orbifold discussion until later in this section.

\subsubsection{Holonomy in string theory I: Narain compactification}

For simplicity, we will consider the heterotic string theory on a rectangular torus. We therefore set the metric on the torus $g_{i j}=\delta_{i j}$, and display the radii $R_{i}$ explicitly. The heterotic NS-NS two-form field $B$ will not enter our considerations so we will set it to zero. The Regge slope $\alpha^{\prime}$ will eventually enter our discussion so we shall keep it explicit. With these conventions, the momenta of the heterotic string are denoted by:

$$
\begin{aligned}
\mathbf{k} & =\left(\mathbf{q}+\sum_{i} w_{i} \mathbf{a}_{i}\right) \sqrt{\frac{2}{\alpha^{\prime}}} \\
k_{i L, R} & =\frac{n_{i}-\mathbf{q} \cdot \mathbf{a}_{i}-\sum_{j} \frac{w_{j}}{2} \mathbf{a}_{i} \cdot \mathbf{a}_{j}}{R_{i}} \pm \frac{w_{i} R_{i}}{\alpha^{\prime}} .
\end{aligned}
$$


In these formulae, no summation is implied unless explicitly stated. The vector $\mathbf{q}$ takes values on the lattice $\Gamma_{8} \oplus \Gamma_{8}$ or $\Gamma_{16}$ for the $E_{8} \times E_{8}$ or the $\operatorname{Spin}(32) / \mathbb{Z}_{2}$ heterotic string, respectively. The $n_{i}$ and $w_{i}$ are integers corresponding to the momentum and winding numbers, respectively. When rescaled by $\sqrt{\alpha^{\prime} / 2}$, these momenta are dimensionless and take values on an even self-dual lattice $\Gamma_{16+d, d}$.

Restricting to states with $w_{i}$ equal to zero, the spectrum exhibited in eqs (6) and (7) is that of compactified gauge theory with holonomies parametrised by $\mathbf{a}_{i}$. We may take the $\mathbf{a}_{i}$ used for a specific compactification of YangMills theory as a starting point for discussing a similar compactification of heterotic string theory. However, the string theory spectrum is richer because states with non-zero winding have to be added to the spectrum.

In the decomposition of eqs (6) and (7), the vectors $\mathbf{k}$ correspond to quantum numbers reflecting the group lattice. In gauge and string theory, the group is broken to subgroups by expectation values for the Wilson lines $\mathbf{a}_{i}$. In gauge theory, the weight lattices for these subgroups correspond to sublattices of the group lattice - an observation which is crucial to the analysis of $[22,24]$. This essential point however is not true for string theory! Winding heterotic strings have bosons on their world-sheet that are charged with respect to the holonomies. Therefore the momenta of these bosons receive corrections reflected in (6). This implies the existence of representations of the gauge group that would not be present in pure gauge theory, where winding modes are absent. In particular, the topology of the unbroken subgroup will be different from the topology that would be deduced from an analysis of the low energy gauge theory. With respect to the use of the phrase "low energy theory," note that the extra representations can be arbitrarily heavy because they arise from strings that may wrap arbitrarily large circles.

Which compactifications feel this difference between gauge and string theory? Those compactifications with holonomies implementing outer auto- 
morphisms are not affected. By construction all $\mathbf{a}_{i}$ should commute with the outer automorphism. This implies that although there will be extra representations, these representations are invariant under the outer automorphism and therefore cannot obstruct the compactification. In particular, the construction of the CHL string in [2] completely parallels the construction in gauge theory described earlier.

For compactifications with twisted boundary conditions, at least one of the $\mathbf{a}_{i}$ is not invariant under the action implemented by one of the $\Theta_{j}[27]$ (with $i \neq j$ ). Instead, we have

$$
\theta_{j}\left(\mathbf{a}_{i}\right)=\mathbf{a}_{i}+\mathbf{z}_{i j} \quad \Leftrightarrow \quad \Theta_{j}^{-1} \exp \left(2 \pi i \mathbf{a}_{i}\right) \Theta_{j} \exp \left(-2 \pi i \mathbf{a}_{i}\right)=\exp \left(2 \pi i \mathbf{z}_{i j}\right) .
$$

This equation implies that $\mathbf{z}_{i j}$ is a vector on the (co)weight ${ }^{4}$ lattice of the original group, since the commutator on the left hand side should be equal to the identity. The group element $\Theta_{j}$ implements a length preserving (orthogonal) automorphism, and hence $\mathbf{a}_{i}$ has the same length as $\theta_{j}\left(\mathbf{a}_{i}\right)$. Putting these facts together, we see that the insertion of winding states not only leads to the introduction of states with group quantum numbers $\mathbf{a}_{i}$, but also automatically generates images for these states. Therefore $\Theta_{j}$ is also a symmetry of the string theory. Although the explicit representation content of string and gauge theory are distinct, the constructions are completely parallel so the arguments used in [17] and [36] are not affected.

The construction of triples $i s$ affected by the extra winding states. Recall that to construct a non-trivial triple, we have to turn on holonomies that leave a non-simply-connected subgroup unbroken, or a subgroup with fundamental group larger than the fundamental group of the original group. The topology of the unbroken subgroup can be deduced from the representation content of the theory, and this is modified by the presence of winding states. We will return to this point momentarily.

For constructions of quadruples and quintuples, it is hard to make a

\footnotetext{
${ }^{4}$ Actually it is the coweight lattice [27], but since we will discuss string theory with simply-laced groups only, we can identify the coweight lattice with the weight lattice.
} 
general statement. The construction of quadruples and quintuples may be viewed as an inductive process, where triples are constructed in an intermediate step. These triple constructions may be restricted by winding states, but one has to check the topology of the gauge (sub)group case by case.

\subsubsection{The topology of subgroups in string theory}

We have seen that some gauge theory vacuum configurations cannot be reproduced in string theory. We will now investigate which configurations remain in $E_{8} \times E_{8}$ and $\operatorname{Spin}(32) / \mathbb{Z}_{2}$ string theory for the specific case of triples. This will provide an alternate derivation of the constraints obtained in section 2.2. Recall that we should look for group elements, to be used as holonomies, with a non-simply-connected centraliser.

Our analysis is based on theorem 1 of [22]. Equivalent results can be found in [24]. This theorem states that any element of a simple group can be conjugated into the form,

$$
\exp \left(2 \pi i \sum_{j=1}^{r} s_{j} \omega_{j}\right),
$$

where $r$ is the rank of the group, and the $\omega_{j}$ are the fundamental coweights of the group. The $s_{j}$ are a set of non-negative numbers satisfying

$$
\sum_{j=0} s_{j} g_{j}=1
$$

with $g_{j}$ the root integers. This last relation determines the number $s_{0}$. The theorem further states that the centraliser of this element is obtained by erasing all nodes $i$ for which $s_{i} \neq 0$ from the extended Dynkin diagram, and adding $U(1)$ factors to complete the rank of the group. The fundamental group $\pi_{1}$ of the centraliser contains $\mathbb{Z}$ factors for the added $U(1)$ factors. In addition, there is a $\mathbb{Z}_{n}$ where $n$ is the greatest common divisor (gcd) of the coroot integers of the erased roots.

For the non-simple group $E_{8} \times E_{8}$ any element can be conjugated to an element that is a product of two elements of the form (9). Since the group is 
simply-laced, we will drop the distinction between root and coroot, weight and coweight. Let us first consider one of the $E_{8}$-factors, setting the group element for the other factor to the identity.

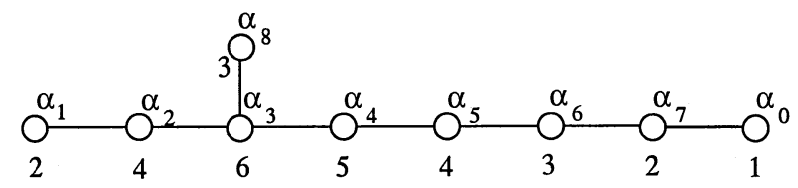

Figure 1: The extended Dynkin diagram of $E_{8}$. The integers are the (co)root integers associated to the respective nodes.

If we desire that the centraliser of an element contains an $m$-fold connected factor with $m \neq 1$, we cannot erase the extended root $\alpha_{0}$ which has root integer 1 . Therefore, we have to set $s_{0}=0$. The extended root $\alpha_{0}$ will now survive as a root of the subgroup. The simple factor of which $\alpha_{0}$ is a root is either $S U(n)$ with $2 \leq n \leq 9$ or $\operatorname{Spin}(16)$.

In heterotic string theory, there are winding states on the weight lattice,

$$
\sum_{j=1}^{8} s_{j} \omega_{j}+\text { roots }
$$

of the unbroken gauge group. We easily find that,

$$
<\sum_{j=1}^{8} s_{j} \omega_{j}, \alpha_{0}>=s_{0}-1=-1,
$$

and therefore $\sum_{j=1}^{8} s_{j} \omega_{j}$ projected onto the subgroup containing $\alpha_{0}$ is minus the weight corresponding to the simple root $\alpha_{0}$ in the unbroken gauge group. Therefore, there is at least one state, with winding number 1 , which transforms in the $\overline{\mathbf{n}}$ irreducible representation (the anti-fundamental representation) of an $S U(n)$ factor, or the $\mathbf{1 6}$ (the vector representation) of $\operatorname{Spin}(16)$. This state is a singlet with respect to other simple factors in the centraliser because $\left\langle\sum_{j=1}^{8} s_{j} \omega_{j}, \alpha_{i}>=s_{i}=0\right.$ when $\alpha_{i}$ is a root of a surviving subgroup. Note that this then implies that the relevant state transforms in a simply-connected representation, unless it transforms in the 
16 of $\operatorname{Spin}(16)$. In this single exceptional case, the vector lattice of $\operatorname{Spin}(16)$ has to be added to a group lattice that already contains a spin weight lattice and the root lattice. Again, the result is the lattice of a simply-connected group.

The conclusion then is that in $E_{8} \times E_{8}$ string theory the holonomies that are trivial in one $E_{8}$ factor do not give non-simply-connected subgroups at all. Closer inspection shows that the only way to get non-simplyconnected subgroups in string theory, is to use holonomies that in gauge theory would break each $E_{8}$ factor to a group containing a semisimple, nonsimply-connected factor. In gauge theory, this would result in a semisimple part with fundamental group $\mathbb{Z}_{n_{1}} \times \mathbb{Z}_{n_{2}}$. An analysis of the group elements that give rise to such a centraliser shows that in string theory, the semisimple part has fundamental group $\mathbb{Z}_{n}$ with $n=\operatorname{gcd}\left(n_{1}, n_{2}\right)$.

It is therefore possible to construct triples in the $E_{8} \times E_{8}$ theory, but of the 144 components of the $E_{8} \times E_{8}$ gauge theory only a set of 12 "diagonal" constructions can be realized in string theory. This is in complete accord with our earlier anomaly cancellation results. A similar analysis can be performed for the $\operatorname{Spin}(32) / \mathbb{Z}_{2}$ string. As the techniques involved are the same as for the $E_{8} \times E_{8}$ heterotic string, we will give fewer details.

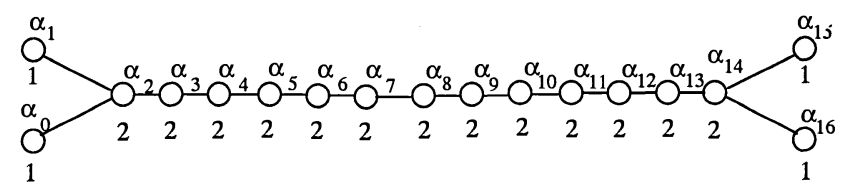

Figure 2: The extended Dynkin diagram of $D_{16}(\operatorname{Spin}(32))$. The integers are the (co)root integers associated to the respective nodes.

As remarked before, the standard compactifications with and without vector structure are not obstructed in any way. Let us first consider the gauge theory triple in $\operatorname{Spin}(32)$ with vector structure. It is not hard to show that it is impossible to construct this triple in string theory because there is no element that gives a centraliser with non-simply-connected semisimple 
part in $\operatorname{Spin}(32)$. There are elements that have a centraliser with nonsimply-connected semisimple part in $\operatorname{Spin}(32) / \mathbb{Z}_{2}$, but this is because the group itself is not simply-connected, and therefore results in compactifications without vector structure rather than a triple with vector structure.

Moving to compactifications without vector structure, let us first note that $\operatorname{Spin}(32) / \mathbb{Z}_{2}$ has a non-trivial center which is isomorphic to $\mathbb{Z}_{2}$. We need two holonomies to encode the absence of vector structure. The third holonomy has to commute with the other two, but is otherwise unrestricted. In particular, if $\Omega$ is an allowed choice, then so is $z \Omega, z$ being the non-trivial centre element in the centre of $\operatorname{Spin}(32) / \mathbb{Z}_{2}$. These two choices are represented as points in two disconnected components which have an identical structure.

The element $z$ is represented by the identity in the vector representation of $\operatorname{Spin}(32)$. In particular, the two components mentioned above cannot be distinguished by their holonomies in $S O(32)$, and have an identical orientifold description [29]. This degeneracy should not persist in a consistent string theory, and indeed it does not. As an example, set $\Omega$ to the identity. Then $\Omega$ has $\operatorname{Spin}(32) / \mathbb{Z}_{2}$ as its centraliser. On the other hand, for $\Omega=z=\exp \left(2 \pi i \mathbf{a}_{i}\right)$, with $\mathbf{a}_{i}$ on the vector weight lattice, the centraliser in gauge theory would also be $\operatorname{Spin}(32) / \mathbb{Z}_{2}$; but in string theory it turns out to be ${ }^{5} \operatorname{Spin}(32)$ - a simply connected group! It therefore destroys the possibility for absence of vector structure. By continuation, we see that the degeneracy is completely lifted, and only one of the degenerate components survives.

For gauge theory on $T^{3}$ with $\operatorname{Spin}(32) / \mathbb{Z}_{2}$ gauge group, and absence of vector structure, two more components exist. One may construct holonomies parametrising these components by the methods of [24] as mentioned earlier, or with an alternative approach [29]. Attempting to construct these holonomies in heterotic string theory does not lead to flat bundles. These

\footnotetext{
${ }^{5}$ We are ignoring the Kaluza-Klein gauge bosons here.
} 
choices therefore are not realized in toroidally compactified string theory.

\subsubsection{Holonomy in string theory II: asymmetric orbifolds}

Having dealt with the intricacies of Narain compactification, we should now implement the discrete transformations $\Theta_{j}$ appearing in equation (4). This can be done by means of the asymmetric orbifold construction $[34,35]$. We will briefly review the general formalism and then apply it to our problem.

Essentially by definition, an asymmetric orbifold uses the fact that the left and right-moving degrees of freedom on the world-sheet are largely independent. The orbifold group can therefore have a different action on the left and right-movers. For heterotic strings, where left and right-movers live on different spaces, this possibility is quite natural. Let us use $P_{L}\left(P_{R}\right)$ to denote the left-moving (right-moving) momenta of the heterotic string. The group elements $g$ of the orbifold group act separately on the left and right-moving momenta since mixing left and right-movers typically leads to inconsistencies. The action of $g$ consists of a combination of a rotation $\theta_{L}$ and a translation $a_{L}$ acting on the left-moving sector. Similarly, a rotation $\theta_{R}$ and translation $a_{R}$ acting on the right-movers. The action of $g$ on states in the Hilbert space takes the form,

$$
g\left|P_{L}, P_{R}\right\rangle=e^{2 \pi i\left(P_{L} \cdot a_{L}-P_{R} \cdot a_{R}\right)}\left|\theta_{L} P_{L}, \theta_{R} P_{R}\right\rangle .
$$

The orbifold construction leads to untwisted and twisted sectors in the theory. In the untwisted sector, describing states invariant under the orbifold action, we encounter (in the partition function) a sum over a lattice $I$ which is the sublattice of $\Gamma_{16+d, d}$ invariant under rotations by $\theta$. In the twisted sector, we find a lattice $I^{*}+a^{*}$, where $I^{*}$ is the lattice dual to $I$ and $a^{*}$ is the orthogonal projection of $a=\left(a_{L}, a_{R}\right)$ onto $I$ [34].

The left- and right-moving sectors of the closed string are not completely independent. The constraint of level matching connects both sectors. This constraint leads to consistency conditions on the asymmetric orbifold. Let 
the group element $g$ have finite order $n$. The eigenvalues of $\theta_{L}$ are then of the form $\exp \left(2 \pi i r_{i} / n\right), i=1, \ldots, 19$, while $\exp \left(2 \pi i s_{i} / n\right), i=1,2,3$ are the eigenvalues of $\theta_{R}$. The consistency conditions for $n$ odd are:

$$
\sum_{i} r_{i}^{2}=\left(n a^{*}\right)^{2} \bmod n
$$

For even $n$, this condition is replaced by a more stringent one. There are supplementary conditions,

$$
\begin{gathered}
\sum_{i} r_{i}^{2}=\left(n a^{*}\right)^{2} \bmod 2 n, \\
\sum_{i} s_{i}=0 \bmod 2, \quad v \theta_{B}^{n / 2} v=0 \bmod 2 .
\end{gathered}
$$

The last condition should hold for any $v \in \Gamma_{16+d, d}$, where $\theta_{B}$ is a block diagonal matrix with $\theta_{L}$ and $\theta_{R}$ on the diagonal.

In our applications, the asymmetric orbifold construction will be used to implement outer automorphisms, or Weyl reflections on the gauge group. Since the gauge group comes from left-moving excitations, we set $\theta_{R}=1$ and drop the subscript on $\theta_{L}=\theta$. This conforms with notation used in previous sections. Notice that the first condition in (14) is trivialized.

The shifts $a_{L, R}$ will be interpreted as physical translations. We therefore take a minimal lightlike vector in a $\Gamma_{1,1}$ sublattice, divide it by $n$, and identify the shifts $\left(a_{L}, a_{R}\right)$ with the components of that resulting vector. There is only one ambiguity in this prescription: there is the possibility of a relative sign between the components $a_{L}$ and $a_{R}$ (there is another overall sign corresponding to a parity transformation). The difference in sign comes from the choice of fractionalizing either the winding numbers, or fractionalizing the momenta. Both choices are related by a T-duality. We shall return to this point later. In the following discussion, we fractionalise the winding numbers because this has an obvious space-time interpretation.

After quotienting string theory by $g$, we obtain a theory with a clear geometric interpretation. Traversing the cycle on the spatial torus in the 
direction of $\left(a_{L}, a_{R}\right)$ gives a holonomy implementing a Weyl reflection or an outer automorphism (see also $[2,36]$ ). We have now gathered all the elements needed to construct the orbifolds.

\subsubsection{Triples in string theory}

In this section, we present an analysis of some non-trivial heterotic compactifications on a 3-torus. For non-trivial compactifications on lower-dimensional tori, we refer the reader to $[2,36]$.

Since we earlier ruled out the existence of triples in $\operatorname{Spin}(32) / \mathbb{Z}_{2}$ string theory, we deal exclusively with the $E_{8} \times E_{8}$ theory in this section. Our previous analysis combined with results in $E_{8}$ gauge theory [19,21-24] lead us to expect non-trivial triples for which one holonomy implements a Weyl reflection generating a cyclic group $\mathbb{Z}_{m}$ with $m=2,3,4,5$ or 6 .

In this section, we will construct asymmetric orbifolds for special choices of holonomy. The extension to the general case will be delayed until section 2.4. Our choice will be to embed the holonomies for the $\mathbb{Z}_{m}$-triples in "minimal" subgroups of $E_{8}$. These are the smallest simply-laced subgroups that contain a $\mathbb{Z}_{m}$-triple. This choice can be motivated along the lines sketched in [22]. The maximal torus of the group has a subtorus $T$ that commutes with the triple. The centraliser of $T$ is a product of $T$ with a semisimple group $C$. This semisimple group $C$ is the "minimal" subgroup which we require. For $E_{8}$ gauge theory, $C=D_{4}, E_{6}, E_{7}, E_{8}$ or $E_{8}$ for the $\mathbb{Z}_{m}$-triple with $m=2,3,4,5$ or 6 , respectively. For $E_{8} \times E_{8}$ string theory, we find that $C=D_{4} \times D_{4}$, etc. The subtorus $T$ corresponds to surviving moduli, so we can interpret the group $C$ as representing eliminated moduli. Our choice is dictated by the desire to make $C$ manifest in the construction. The entries from the list of possible $C^{\prime}$ 's will reappear later in our paper.

The holonomies are essentially fixed by the decision to embed them in a "minimal" subgroup. The only remaining freedom corresponds to global 
gauge transformations, or equivalently, lattice symmetries of the heterotic string. Triples embedded in these subgroups have a number of convenient properties. One is that all three holonomies are conjugate to each other [24], which implies that they have the same set of eigenvalues in every representation of the gauge group. Further, from the minimality property, it follows that these eigenvalues are of the form $\exp (2 \pi i n / m)$ where $n \in \mathbb{Z}[21,23]$. This is convenient for checking the asymmetric orbifold consistency conditions (12), (13) and (14). Additional properties will emerge in the construction.

Let us now specify values for some of the quantities appearing in the formulae for the momenta, (6) and (7). We work on a 3 -torus so $i=1,2,3$. We will turn on holonomies in the 1 and 2-directions, and use direction 3 for the shift accompanying the orbifold projection. The holonomy in this direction, $\mathbf{a}_{3}$, will be set to zero for the moment. In equation (7), the inner products $\mathbf{a}_{i} \cdot \mathbf{a}_{j}$ appear. Since the holonomies at the relevant point in moduli space are conjugate to each other, we see that

$$
\mathbf{a}_{1}^{2}=\mathbf{a}_{2}^{2}
$$

Let us introduce the notation $\mathbf{a}_{i}=\left(\tilde{a}_{i}^{I}, \tilde{a}_{i}^{I I}\right)$ to display the Wilson lines in the 'first' (I), and 'second' (II) $E_{8}$ factor. It is convenient to set $\mathbf{a}_{1}=\left(\tilde{a}_{1}, \tilde{a}_{1}\right)$ and $\mathbf{a}_{2}=\left(\tilde{a}_{2},-\tilde{a}_{2}\right)$. This eliminates the inner product $\mathbf{a}_{1} \cdot \mathbf{a}_{2}$ from our formulae, leaving only diagonal terms in the spatial momenta. We will use an orbifold projection that is symmetric in both $E_{8}$ factors. Notice that in this way, we implement the prescription that the contributions of each $E_{8}$ factor to the Chern-Simons invariant cancel each other. There are other ways of implementing this constraint; for example, by choosing $\mathbf{a}_{1}$ and $\mathbf{a}_{2}$ symmetric in both factors, and choosing opposite orbifold projections in the two $E_{8}$ 's. This would leave us with off-diagonal terms in the momenta, and we consider this less convenient. Nevertheless, it should provide equivalent results.

The value of $\mathbf{a}_{1}^{2}=\mathbf{a}_{2}^{2}$ can be found in various ways. In the setup we have 
chosen, the holonomy parametrized by $\mathbf{a}_{1}$ eliminates only one node from each $E_{8}$ extended Dynkin diagram, and from the discussion around eq. (9), it follows that $\mathbf{a}_{1}$ is of the form $\left(\omega_{j} h_{j}^{-1}, \omega_{j} h_{j}^{-1}\right)$. Here $\omega_{j}$ is the (co)weight and $h_{j}$ the (co)root integer associated to the node. ${ }^{6}$ We now easily find $\mathbf{a}_{i}^{2}$ by noting that the weight can be expanded in the simple roots,

$$
\omega_{i}=\sum_{k} p_{i}^{k} \alpha_{k}
$$

It is then trivial to show that $\mathbf{a}_{i}^{2}=2 p_{i}^{i} /\left(h_{i}\right)^{2}$ (no summation implied), where $p_{i}^{i}$ is a diagonal element of the inverse Cartan matrix. For the cases under consideration, we find that $\mathbf{a}_{i}^{2}=2(m-1) / m .^{7}$

Combining these conventions and results, we find the momenta for the compactified heterotic string before the orbifold projection:

$$
\begin{aligned}
\mathbf{k} & =\left(\mathbf{q}+\sum_{i=1,2} w_{i} \mathbf{a}_{i}\right) \sqrt{\frac{2}{\alpha^{\prime}}} \\
k_{i L, R} & =\frac{m n_{i}-m \mathbf{q} \cdot \mathbf{a}_{i}-w_{i}(m-1)}{m R_{i}} \pm \frac{w_{i} R_{i}}{\alpha^{\prime}}, \quad i=1,2 \\
k_{3 L, R} & =\frac{n_{3}}{R_{3}} \pm \frac{w_{3} R_{3}}{\alpha^{\prime}} .
\end{aligned}
$$

Because of the eigenvalues of the holonomies, $\mathbf{q} \cdot \mathbf{a}_{i}$ is always a multiple of $1 / m$. Therefore, the combination $m n_{i}-m \mathbf{q} \cdot \mathbf{a}_{i}-w_{i}(m-1)$ is always an integer, and actually can take any integer value.

We have now arrived at the point where we want to perform the orbifold construction. From the gauge theory interpretation of the theory, we should be confident that orbifolding will lead to a consistent theory. Nevertheless, we shall verify that the theory given by equations (15), (16) and (17) has the right symmetries, and that the orbifold operation obeys the consistency conditions (12), (13) and (14).

\footnotetext{
${ }^{6}$ For $m<5$, there seem to be more options but only one corresponds to a minimal triple.

${ }^{7}$ It can be proven that this is the minimal value that $\mathbf{a}_{i}^{2}$ can have for an $m$-triple. This provides another invariant way of characterising these holonomies.
} 
The orbifolding operation consists of a shift $a$, and a transformation $\theta$ acting on the gauge part of the lattice. The transformation $\theta$ is an element of the Weyl group of $E_{8} \times E_{8}$. Since the rank of $E_{8} \times E_{8}$ is 16, the Weyl group is a discrete subgroup of the orthogonal group $O(16)$. As discussed before, the requirement of commuting holonomies does not necessarily mean that $\theta\left(\mathbf{a}_{i}\right)$ is equal to $\mathbf{a}_{i}$, but rather implies the weaker condition (8) where $\mathbf{z}_{i j}$ is some lattice vector. There is some ambiguity in the choice of $\mathbf{z}_{i j}$, but in the cases of non-trivial commuting triples, the lattice vector cannot be set equal zero. The choice $\mathbf{z}_{i j}=0$ corresponds to a trivial triple, which should be equivalent to a conventional Narain compactified theory.

To see that the lattice has the right symmetry, we construct the image of a vector with labels $\left(\mathbf{q}, n_{i}, w_{i}, n_{3}, w_{3}\right)$. There should exist a vector labelled by $\left(\mathbf{q}^{\prime}, n_{i}^{\prime}, w_{i}^{\prime}, n_{3}^{\prime}, w_{3}^{\prime}\right)$ with $\mathbf{q}^{\prime}=\theta\left(\mathbf{q}+w_{1} \mathbf{a}_{1}+w_{2} \mathbf{a}_{2}\right)$. We expect existence for generic radii of the spacial torus which implies $w_{i}=w_{i}^{\prime}, w_{3}=w_{3}^{\prime}$ and $n_{3}=n_{3}^{\prime}$. We are therefore led to the equations,

$$
\begin{aligned}
\mathbf{q}^{\prime} & =\theta(\mathbf{q})+w_{1}\left(\theta\left(\mathbf{a}_{1}\right)-\mathbf{a}_{1}\right)+w_{2}\left(\theta\left(\mathbf{a}_{2}\right)-\mathbf{a}_{2}\right), \\
n_{i}^{\prime}-\mathbf{q}^{\prime} \cdot \mathbf{a}_{i} & =n_{i}-\mathbf{q} \cdot \mathbf{a}_{i} \quad i=1,2 .
\end{aligned}
$$

Equation (18) is consistent by construction since both the left and right hand side contain lattice vectors only. We still have to verify that $\left(\mathbf{q}^{\prime}-\mathbf{q}\right) \cdot \mathbf{a}_{i}$ is an integer for $i=1,2$. We will show that both $(\theta(\mathbf{q})-\mathbf{q}) \cdot \mathbf{a}_{i}$ and $\left(\theta\left(\mathbf{a}_{i}\right)-\mathbf{a}_{i}\right) \cdot \mathbf{a}_{j}$ are integers, and hence (19) always has a solution.

The quantity $\left(\theta\left(\mathbf{a}_{i}\right)-\mathbf{a}_{i}\right) \cdot \mathbf{a}_{j}$ is actually zero for $i \neq j$ because of the specific choice of $\mathbf{a}_{1}, \mathbf{a}_{2}$, and because $\theta$ is symmetric in both $E_{8}$ factors. We remarked previously that $\theta\left(\mathbf{a}_{i}\right)-\mathbf{a}_{i}=\mathbf{z}_{i}$ for some lattice vector $\mathbf{z}_{i}$. Then $\left(\theta\left(\mathbf{a}_{i}\right)\right)^{2}=\mathbf{a}_{i}^{2}=\mathbf{z}_{i}^{2}+2 \mathbf{a}_{i} \cdot \mathbf{z}_{i}+\mathbf{a}_{i}^{2}$, where use was made of the fact that $\theta \in O(16)$. Since $\mathbf{z}_{i}$ is on an even lattice, it immediately follows that $\mathbf{a}_{i} \cdot \mathbf{z}_{i}$ is an integer. Finally, rewriting $(\theta(\mathbf{q})-\mathbf{q}) \cdot \mathbf{a}_{i}$ as $\left(\theta^{-1}\left(\mathbf{a}_{\mathbf{i}}\right)-\mathbf{a}_{\mathbf{i}}\right) \cdot \mathbf{q}$, we notice that this is an inner product between two lattice vectors, and hence also integer. Therefore an image point always exists.

For the orbifold consistency conditions (12) and (13), we need the eigen- 


\begin{tabular}{|c|c|c|c|c|c|c|c|c|c|c|c|c|c|c|c|}
\hline$m$ & 2 & \multicolumn{3}{|c|}{3} & \multicolumn{3}{|c|}{4} & \multicolumn{5}{c|}{5} & \multicolumn{5}{c|}{6} \\
\hline$r_{i}$ & 1 & 1 & 2 & 1 & 2 & 3 & 1 & 2 & 3 & 4 & 1 & 2 & 3 & 4 & 5 \\
\hline multiplicity & 8 & 6 & 6 & 4 & 6 & 4 & 4 & 4 & 4 & 4 & 2 & 4 & 4 & 4 & 2 \\
\hline
\end{tabular}

Table 4: Eigenvalues $r_{i} \neq 0$ for the $\mathbb{Z}_{m}$ orbifolds

values $r_{i}$ of $\theta$. These can be obtained from group theory [21-24] and are listed in table 4 . We set the shift $a$ to a light-like vector so $\left(a^{*}\right)^{2}$ is always zero. In all cases, the orbifold consistency conditions are satisfied. For future use, we remark that the eigenvalues and multiplicities appearing in table 4 are identical to the eigenvalues and multiplicities of automorphisms K3 [37].

The last condition that we need to check is the second condition of eq. (14). It can be shown that this leads to the same condition for $n=2,4,6$ (compare with table 4 ). In all cases $\theta_{B}^{n / 2}$ is a matrix that reflects 8 orthogonal roots. It is then easily checked that (14) is satisfied.

With the table of eigenvalues, it is also easy to calculate the zero-point energies for the twisted sector(s). An eigenvalue $r_{i}$ contributes,

$$
\frac{1}{48}-\frac{1}{16}\left(2 \frac{r_{i}}{m}-1\right)^{2}
$$

to the zero-point energy. A periodic boson has $r_{i}=0$, and hence contributes $-\frac{1}{24}$. Summing all contributions leads to a remarkably simple result: the zero point energies are $-1 / m$ for the twisted sector(s) of the $\mathbb{Z}_{m}$-orbifold. With all the requirements checked, we found-as expected - that the asymmetric orbifolds are consistent.

\subsubsection{Anomaly cancellation and winding states}

In the previous section we barely mentioned the Chern-Simons invariant, which provides another way to decide which orbifolds are consistent. Nevertheless, both orbifold analysis and the Chern-Simons analysis lead to identical results. Let us examine the relation between the approaches in more 
detail.

According to our analysis of the topology of subgroups in string theory, it is the presence of winding states that rules out particular gauge theory compactifications in string theory. Let us consider such a state with $w_{1}=$ 1 and $w_{2}=w_{3}=0, \mathbf{q}=0$. We have seen that such a state carries a gauge group representation vector equal to $\mathbf{a}_{1}$. We denote this state by $\left|\mathbf{a}_{1}\right\rangle$. Consider parallel transport of this state along the following path: we start by going around a closed cycle in the 2 direction then a closed cycle in the 3 direction, around the 2 direction with the opposite orientation, then around the 3 direction with the opposite orientation. Because of the background gauge fields, the state transforms in the following way,

$$
\begin{aligned}
& \left|\mathbf{a}_{1}\right\rangle \quad \rightarrow \quad e^{2 \pi i\left(\mathbf{a}_{1} \cdot \mathbf{a}_{2}\right)}\left|\mathbf{a}_{1}\right\rangle \quad \rightarrow \quad e^{2 \pi i\left(\mathbf{a}_{1} \cdot \mathbf{a}_{2}\right)}\left|\theta\left(\mathbf{a}_{1}\right)\right\rangle \\
& \rightarrow e^{2 \pi i\left(\left(\mathbf{a}_{1}-\theta\left(\mathbf{a}_{1}\right)\right) \cdot \mathbf{a}_{2}\right)}\left|\theta\left(\mathbf{a}_{1}\right)\right\rangle \rightarrow e^{2 \pi i\left(\left(\mathbf{a}_{1}-\theta\left(\mathbf{a}_{1}\right)\right) \cdot \mathbf{a}_{2}\right)}\left|\mathbf{a}_{1}\right\rangle .
\end{aligned}
$$

With the results from [24], the Chern-Simons invariant can be expressed in terms of the gauge fields as $\left(\theta\left(\mathbf{a}_{1}\right)-\mathbf{a}_{1}\right) \cdot \mathbf{a}_{2}$. On the other hand, we transported a state around a contractible curve in a flat background, and consistency requires that the final phase factor appearing in (21) equal unity. The conclusion is that the Chern-Simons invariant must be integer, precisely as was argued in section 2.2. Analogous arguments apply to other winding states.

To complete the connection, we remark that (21) only expresses the change in phase caused by the gauge fields. The state $\left|\mathbf{a}_{1}\right\rangle$ is due to a winding string, and in the transport process sketched above, it sweeps out a two-dimensional world sheet. It therefore also picks up another contribution $\exp \left(2 \pi i \int B\right)$ to the phase, where the integral is over the world sheet area sweeped out. The surface integral can be converted to a volume integral giving the total phase change:

$$
\exp \left(2 \pi i \int\{d B-C S(A)\}\right)=\exp \left(2 \pi i \int H\right) .
$$

The right hand side states that the total phase change should be attributed 
to the gauge field strength to which the string couples. This equation is just a global version of anomaly cancellation (2).

\subsection{Moduli spaces}

Our previous discussion focused on orbifold descriptions appearing at specific points in the orbifold moduli space. In this section, we extend the discussion to cover the whole moduli space of asymmetric orbifold theories.

\subsubsection{Lattices for the orbifolds}

In the standard toroidal or Narain compactification of the heterotic string, a central role is played by the even self-dual lattice $\Gamma_{d+16, d}[32,33]$. The momenta lie on this lattice. In the construction of the moduli space for a Narain compactification, we further divide out by a discrete subgroup corresponding to the symmetries of $\Gamma_{d+16, d}$. In an attempt to set up a similar structure for the CHL string and its compactifications, Mikhailov introduced lattices for these theories [38]. In a somewhat more laborious construction, the same can be done for the asymmetric orbifolds of the previous section.

Recall that for all orbifolds corresponding to triples, we had a transformation $\theta$ which has order $m$. For each $\theta$, we can define a projection $P_{\theta}$ acting on $\mathbb{R}_{3,19}$ by,

$$
P_{\theta}=\frac{1}{m} \sum_{n=0}^{m-1} \theta^{n} .
$$

From $\theta P_{\theta}=P_{\theta} \theta=P_{\theta}$, we see that $P_{\theta}$ projects all lattice vectors in $\Gamma_{3,19}$ onto the space invariant under $\theta$. In particular, for the holonomies introduced in the previous section we have $P_{\theta}\left(\mathbf{a}_{i}\right)=0$.

As our starting point, we return to the momenta (15), (16) and (17) of the heterotic string prior to orbifolding. In the orbifolded theory, the untwisted sector consists of those states that are left invariant under the 
projection. These are of the form

$$
N \sum_{n=0}^{m-1} \exp (2 \pi i n a \cdot p)\left|\theta^{n}(\mathbf{k}), p\right\rangle .
$$

Here, we symbolically denote the spatial momenta by $p$, the group quantum numbers by $\mathbf{k}$, while $a$ and $\theta$ are the shift and rotation of the orbifold symmetry. There is also a normalization constant $N$.

To these states, we associate a lattice in the following way: first we define

$$
\mathbf{q}_{i n v}=\sqrt{m} P_{\theta}(\mathbf{q})
$$

where the reason for the factor of $\sqrt{m}$ will soon become clear. We also set

$$
\tilde{n}_{i}=m n_{i}-m \mathbf{q} \cdot \mathbf{a}_{i}-w_{i}(m-1)
$$

for $i=1,2$. We will rescale the radii for the 1 and 2 directions by defining $R_{i}^{\prime}=m R_{i}$. We also define $\alpha^{\prime \prime}=m \alpha^{\prime}$. Note that the invariant radii $R_{i} / \sqrt{\alpha^{\prime}}$ are only rescaled by a factor $\sqrt{m}$.

Now define a lattice by projecting the Narain lattice (15), (16) and (17) onto the invariant subspace of $\theta$. We call this lattice $\tilde{I}$. With the reparametrisations introduced above, its vectors are given by:

$$
\begin{aligned}
\mathbf{v} & =\left(\mathbf{q}_{i n v}\right) \sqrt{\frac{2}{\alpha^{\prime \prime}}}, \\
v_{i L, R} & =\frac{\tilde{n}_{i}}{R_{i}^{\prime}} \pm \frac{w_{i} R_{i}^{\prime}}{\alpha^{\prime \prime}}, \quad i=1,2 \\
v_{3 L, R} & =\frac{n_{3}}{R_{3}} \pm \frac{m w_{3} R_{3}}{\alpha^{\prime \prime}} .
\end{aligned}
$$

The vectors $v_{i L, R}$ and $v_{3 L, R}$ form a lattice, which when rescaled by $\sqrt{\alpha^{\prime \prime} / 2}$ may be called $\Gamma_{2,2} \oplus \Gamma_{1,1}(m)$. Here we follow the notation of Mikhailov [38], defining the lattice $\Gamma_{1,1}(m)$ to be a lattice of signature $(1,1)$ generated by 2 vectors $e$ and $f$ with scalar product $(e \cdot f)=m$. For a summary of our lattice conventions, see Appendix A. This lattice arises as an intermediate step because we have not yet included the twisted sectors. 
The vectors $\mathbf{v}$ are the vectors of $\Gamma_{8} \oplus \Gamma_{8}$ projected onto the subspace invariant under $\theta$ and suitably rescaled. This defines a different lattice for every $m$, which can be deduced from group theory. The lattices are $D_{4} \oplus D_{4}$, $A_{2} \oplus A_{2}, A_{1} \oplus A_{1}$ for $m=2,3,4$, respectively. The lattice for the cases $m=5$ and $m=6$ is the empty lattice. These lattices are usually defined so their roots are normalized with length $\sqrt{2}$. In the symmetry groups which arise in gauge theory, these form the short roots of non-simply-laced algebras at level 1. For example, at the point in moduli space constructed here the gauge group is $F_{4} \times F_{4}$ for the $m=2$ case, and $G_{2} \times G_{2}$ for the $m=3$ case, with long roots which have length 2 and $\sqrt{6}$, respectively. The gauge group $S U(2)$ in the $m=4$ case has roots of length $\sqrt{8}$ (it is at level 4 ). The vectors with length $\sqrt{2}=\sqrt{8} / 2$ are on the weight lattice of $S U(2)$. Although there is no simple 4-laced algebra, there is a 4-laced affine Dynkin diagram that plays a role in the description of the group theory [24]. Interestingly, the lattices of $F_{4}, G_{2}$ and $A_{1}$ at level 4 all satisfy a 'generalised self-duality' in the sense that their weight lattice is identical to the original lattice. For the simply-laced $E_{8}$ lattice, this notion of 'generalised self-duality' coincides with self-duality. There is an interesting connection between this observation and S-duality of four-dimensional theories, which we shall discuss later.

In the twisted sectors, the momenta lie on the lattice $I^{*}$, which is dual to the lattice $I$ of invariant vectors. As in the case of the untwisted sector, we will treat the parts of the lattices that represent the group quantum numbers, and the part that represents the space quantum numbers separately.

Obviously the lattice $I$ of invariant vectors is a sublattice of the lattice $\tilde{I}$. It can be verified that the group part of the lattice $I$ of invariant vectors is the lattice which we will denote $\sqrt{2}\left(D_{4}^{*} \oplus D_{4}^{*}\right), \sqrt{3}\left(A_{2}^{*} \oplus A_{2}^{*}\right), 2\left(A_{1}^{*} \oplus A_{1}^{*}\right)$ for $m=2,3,4$, respectively. As usual, the star denote the dual lattice which is, of course, the (co)weight lattice. The stars arise because we define the lattices $I$ relative to the lattices $\tilde{I}$, which forces us to keep track of relative orientations. It is now trivial to construct the group part of the lattices $I^{*}$ : these are $\left(D_{4} \oplus D_{4}\right) / \sqrt{2},\left(A_{2} \oplus A_{2}\right) / \sqrt{3},\left(A_{1} \oplus A_{1}\right) / 2$, for $m=2,3,4$. 
We now construct the spatial part of the invariant lattice $I$. First note that for invariant vectors,

$$
P_{\theta}\left(\mathbf{q}+\sum_{i} w_{i} \mathbf{a}_{i}\right)=P_{\theta}(\mathbf{q})=\mathbf{q}+\sum_{i} w_{i} \mathbf{a}_{i}
$$

Since $P_{\theta}(\mathbf{q})$ is a sum of elements of the root lattice, it again lies on the root lattice. It then follows that $w_{i} \mathbf{a}_{i}$ is on the root lattice. Because $\mathbf{a}_{i}$ is on the weight lattice, we deduce that for invariant vectors, $w_{i}$ has to be a multiple of $m$, say $l_{i} m$. Another way to see this is from the value of $\mathbf{a}_{i}^{2}=2(m-1) / m$. Also, if $\mathbf{q}+\sum w_{i} \mathbf{a}_{i}$ is on the invariant lattice then its dot product with either $\mathbf{a}_{j}$ has to vanish:

$$
\left(\mathbf{q}+\sum_{i} w_{i} \mathbf{a}_{i}\right) \cdot \mathbf{a}_{j}=P_{\theta}\left(\mathbf{q}+\sum_{i} w_{i} \mathbf{a}_{i}\right) \cdot \mathbf{a}_{j}=\left(\mathbf{q}+\sum_{i} w_{i} \mathbf{a}_{i}\right) \cdot P_{\theta}\left(\mathbf{a}_{j}\right)=0 .
$$

This leads immediately to $\mathbf{q} \cdot \mathbf{a}_{i}=-2 l_{i}(m-1)$. The spatial momenta on the invariant lattice are thus given by,

$$
\frac{n_{i}+l_{i}(m-1)}{R_{i}} \pm \frac{m l_{i} R_{i}}{\alpha^{\prime}}, \quad i=1,2 \quad \frac{n_{3}}{R_{3}} \pm \frac{w_{3} R_{3}}{\alpha^{\prime}} .
$$

Note that $n_{i}+l_{i}(m-1)$ can take any integer value, while $l_{i} m$ is always a multiple of $m$. The momenta on the dual to the spatial part of the invariant lattice are then given by the vectors,

$$
\frac{n_{i}^{\prime}}{m R_{i}} \pm \frac{w_{i}^{\prime} R_{i}}{\alpha^{\prime}}, \quad i=1,2 \quad \frac{n_{3}^{\prime}}{R_{3}} \pm \frac{w_{3}^{\prime} R_{3}}{\alpha^{\prime}} .
$$

We complete the construction of $I^{*}$ with the same reparametrisations as in the untwisted sector: multiply the group parts of the lattices by $\sqrt{m}$, define $R_{i}^{\prime}=m R_{i}$ for $i=1,2$ and set $\alpha^{\prime \prime}=m \alpha^{\prime}$. Note that in all cases, we have the simple result $\tilde{I}=I^{*}$, confirming a result from Appendix A of [34] for our specific case.

To construct the twisted sectors, we still need the shift $a^{*}$. It is given by multiples of

$$
\Delta v_{3 L, R}= \pm \frac{R_{3}}{m \alpha^{\prime}}= \pm \frac{R_{3}}{\alpha^{\prime \prime}}
$$


We remind the reader that the sign choice between the left and right-moving parts of the shift reflects our choice of fractionalizing the winding numbers. Because the lattices $I^{*}$ are identical to $\tilde{I}$, and because the momenta in the $n^{\text {th }}$ twisted sector are given by $I^{*}+n \Delta v_{3 L, R}$ with $n=1, \ldots,(m-1)$, we can assemble the lattices into a single lattice $\Lambda$. In the process of assembly, the spatial part of the lattice is completed to $\Gamma_{3,3}$.

We only computed the lattices for very specific orbifolds with special values of the holonomies and other background fields. To extend to the general case, first note that the metric and antisymmetric tensor field did not play any role so far, and the moduli corresponding to these fields survive the orbifold projection. For the holonomies, we took special values that had

$$
P_{\theta}^{\perp}\left(\mathbf{a}_{i}\right)=\left(1-P_{\theta}\right)\left(\mathbf{a}_{i}\right)=\mathbf{a}_{i} .
$$

For the general case, we take holonomies parametrized by $\mathbf{a}_{i}^{\prime}$, subject to

$$
P_{\theta}^{\perp}\left(\mathbf{a}_{i}^{\prime}\right)=\mathbf{a}_{i} .
$$

The possible moduli for varying the holonomies are then given by $P_{\theta}\left(\mathbf{a}_{i}^{\prime}\right)$. We may use general formulae from $[32,33,39]$ to show that this results in a moduli space that locally has the form,

$$
O(19-\Delta r, 3) /(O(19-\Delta r) \times O(3)),
$$

where $\Delta r$ is the rank reduction for the $\mathbb{Z}_{m}$ orbifold.

As usual, we should also divide on the left by a discrete group of lattice symmetries. Following Mikhailov [38], we propose that this discrete group is formed by the symmetries of the lattice $\Lambda$ constructed above. This is a non-trivial statement with regard to those symmetries in $\Lambda$ that connect different twisted and untwisted sectors. Mikhailov demonstrates this explicitly for his lattice. For our cases, we will not attempt to prove this. However, we note that from a gauge theory point of view, all holonomies are on equal footing (and in special situations, even conjugate to each other). We stress that the asymmetry in our treatment of the various holonomies is purely 
technical, caused by the fact that we are working at the level of the algebra rather than the group. There is therefore every reason to expect symmetry transformations that connect the various sectors, and lift the apparent asymmetry between the holonomies.

We therefore propose that the moduli space of these asymmetric orbifolds is given by,

$$
O(\Lambda) \backslash O(19-\Delta r, 3) /(O(19-\Delta r) \times O(3)) .
$$

The lattices $\Lambda$ and the rank reduction $\Delta r$ are collected in table 5 , where other relevant results are summarized. We have included the standard Narain compactification, denoted by $\mathbb{Z}_{1}$, which fits perfectly in the picture when we take trivial holonomies and $m=1$. In a separate column, we list the lattices $\Lambda^{\perp}$ which are the lattices of vectors orthogonal to the $\Lambda$ sublattice of $\Gamma_{19,3}$. Not surprisingly, the lattices are those of the 'minimal subgroups' $C$ for $m$-triples.

\begin{tabular}{|c||c|c|c|c|}
\hline & $\Lambda$ & $\Lambda^{\perp}$ & $\Delta r$ & $E_{t}$ \\
\hline \hline $\mathbb{Z}_{1}$ & $\Gamma_{3,3} \oplus E_{8} \oplus E_{8}$ & $\emptyset$ & 0 & -1 \\
$\mathbb{Z}_{2}$ & $\Gamma_{3,3} \oplus D_{4} \oplus D_{4}$ & $D_{4} \oplus D_{4}$ & 8 & $-1 / 2$ \\
$\mathbb{Z}_{3}$ & $\Gamma_{3,3} \oplus A_{2} \oplus A_{2}$ & $E_{6} \oplus E_{6}$ & 12 & $-1 / 3$ \\
$\mathbb{Z}_{4}$ & $\Gamma_{3,3} \oplus A_{1} \oplus A_{1}$ & $E_{7} \oplus E_{7}$ & 14 & $-1 / 4$ \\
$\mathbb{Z}_{5}$ & $\Gamma_{3,3}$ & $E_{8} \oplus E_{8}$ & 16 & $-1 / 5$ \\
$\mathbb{Z}_{6}$ & $\Gamma_{3,3}$ & $E_{8} \oplus E_{8}$ & 16 & $-1 / 6$ \\
\hline
\end{tabular}

Table 5: Lattices $\Lambda$, complements $\Lambda^{\perp}$, rank reduction $\Delta r$ and zero-point energies in the twisted sector $E_{t}$ for the $Z_{m}$ asymmetric orbifolds corresponding to triples.

We note that the entries in the $\mathbb{Z}_{2}$ row are identical to those for the CHL string: the same Mikhailov lattice, the same rank reduction and the same zero-point energy. It can indeed be proven that the $\mathbb{Z}_{2}$-triple and the CHL string are equivalent. We will encounter these and many other dualities 
in the next section. To conclude this discussion of the moduli spaces for these compactifications, let us make a preliminary count of the number of distinct 7-dimensional heterotic compactifications that we have constructed. We begin by noting an obvious discrete symmetry: for example in the $\mathbb{Z}_{3}$ case, we can embed a bundle with $C S=1 / 3$ in one $E_{8}$ factor and a bundle with $C S=2 / 3$ in the other $E_{8}$. Flipping the choice of embedding does not generate a new theory. There is a single theory associated to the $\mathbb{Z}_{3}$ orbifold. This counting gives us a single component for $\mathbb{Z}_{1}, \mathbb{Z}_{2}, \mathbb{Z}_{3}, \mathbb{Z}_{4}$ and $\mathbb{Z}_{6}$. At first sight, the case of $\mathbb{Z}_{5}$ seems to give two distinct embeddings $\left(\frac{1}{5}, \frac{4}{5}\right)$ and $\left(\frac{2}{5}, \frac{3}{5}\right)$. However, the two theories are actually equivalent. This can be argued directly using discrete gauge symmetries, but is more easily seen in the dual description which we shall meet in section 4 . Therefore, we find 6 distinct components in the moduli space.

\subsubsection{Dualities}

It is well known that the heterotic $E_{8} \times E_{8}$ string compactified on a circle is equivalent to the heterotic $\operatorname{Spin}(32) / \mathbb{Z}_{2}$ string compactified on a circle. This may be deduced from the form of their moduli spaces [32], and can be made explicit by constructing a map between the two theories at a particular point in the moduli space. The rest of the moduli space is covered by continuation [39]. This duality can be shown to imply a duality between the CHL string, and a compactification of the $\operatorname{Spin}(32) / \mathbb{Z}_{2}$ string without vector structure $[17,36]$, which are $\mathbb{Z}_{2}$-asymmetric orbifolds of the $E_{8} \times E_{8}$ string and the $\operatorname{Spin}(32) / \mathbb{Z}_{2}$ string, respectively.

We now present a list of dualities between heterotic theories with various bundles. The previously mentioned duality between the CHL string and the $\operatorname{Spin}(32) / \mathbb{Z}_{2}$ compactification without vector structure is included as part of a much larger chain. We also find new dualities for theories with rank reduction bigger than 8 . These dualities should be expected on general grounds, such as the structure of the orbifold groups and the moduli spaces 
of various heterotic asymmetric orbifolds. A more detailed study of T-duality for the heterotic string, performed in appendix B, gives us tools that allow us to make these statements more precise.

\section{The $\mathbb{Z}_{2}$-chain}

Our first example starts with the CHL string and will feature toroidal compactifications down to 5 dimensions. We take the heterotic $E_{8} \times E_{8}$ string compactified on a circle of radius $R_{1}$. We will use standard coordinates for the $E_{8} \times E_{8}$ lattice, giving 16 numbers $u_{i} i=1, \ldots, 16$ of which the first 8 denote the first $E_{8}$ factor, and the second group of 8 denotes the other $E_{8}$ factor. The construction from [2] involves modding out this theory by a shift over $\pi R_{1}$ combined with the following transformation on the group lattice:

$$
\theta\left(u_{1}, \ldots, u_{16}\right)=-\left(u_{16}, \ldots, u_{1}\right)
$$

The transformation $\theta$ interchanges the two $E_{8}$ factors so we end up with a theory with gauge group $\left(E_{8}\right)_{2}$ on a circle with radius $R_{1} / 2$. The extra subscript denotes that the gauge group is at level 2.

We compactify this theory on a second circle with radius $R_{2}$. We may turn on a holonomy provided it is invariant under $\theta$. Therefore, we can smoothly deform the theory and introduce a holonomy parametrised by,

$$
\mathbf{a}_{2}=\left(1,0^{14},-1\right)
$$

The notation $0^{14}$ denotes 14 subsequent entries of zero. Introducing this holonomy breaks the gauge group to $\operatorname{Spin}(16)_{2}$.

This theory is interpreted as the CHL string on a torus with radii

$$
\left(R_{1} / 2, R_{2}\right)
$$

We can now find an element of the T-duality group that inverts $R_{2}$ (for details, see appendix B) to obtain a compactification of the $\operatorname{Spin}(32) / \mathbb{Z}_{2}$ 
theory on a torus with radii $\left(R_{1} / 2, R_{2}^{\prime}=\alpha^{\prime} / 2 R_{2}\right)$ with holonomy given by,

$$
\mathbf{a}_{2}^{\prime}=\left(\left(-\frac{1}{2}\right)^{8}, 0^{8}\right) \text {. }
$$

Of course, the gauge group is still $\operatorname{Spin}(16)_{2}$. The vector $\mathbf{a}_{2}^{\prime}$ is no longer invariant under $\theta$. However, we note that

$$
\theta\left(\mathbf{a}_{2}^{\prime}\right)-\mathbf{a}_{2}^{\prime}=\left(\frac{1}{2}^{16}\right)
$$

which is allowed since $\left(\frac{1}{2}^{16}\right)$ is a lattice vector of the $\operatorname{Spin}(32) / \mathbb{Z}_{2}$ lattice. That it lies on the spin-weight lattice indicates that we are dealing here with a compactification without vector structure. We have rederived the result of [36], which was also discussed in [17].

We compactify this theory on a third circle with radius $R_{3}$, and turn on a holonomy parametrised by

$$
\mathbf{a}_{3}=\left(\frac{1}{2}^{2},\left(-\frac{1}{2}\right)^{2}, 0^{8}, \frac{1}{2}^{2},\left(-\frac{1}{2}\right)^{2}\right) .
$$

This breaks the group to $\left(\operatorname{Spin}(8)_{2}\right)^{2}$. We have chosen $\mathbf{a}_{3}$ to be invariant under $\theta$, and $\mathbf{a}_{2}^{\prime} \cdot \mathbf{a}_{3}=0$. We can dualize again in the 3 direction to obtain an $E_{8} \times E_{8}$ theory on a 3 -torus with radii $\left(R_{1} / 2, R_{2}^{\prime}, R_{3}^{\prime}\right)$ (with $\left.R_{i}^{\prime}=\alpha^{\prime} / 2 R_{i}\right)$, and holonomies given by $\mathbf{a}_{2}^{\prime},(32)$, and

$$
\mathbf{a}_{3}^{\prime}=\left(-1,0^{3}, 1,0^{11}\right) \text {. }
$$

Along the first circle, there is still the action of $\theta$ given by (30). However, in the present $E_{8} \times E_{8}$ theory, the root lattice is organized differently. Here one of the $E_{8}$ root lattices is denoted by the 8 coordinates $u_{i}$ with $i=$ $5, \ldots, 12$, while the second $E_{8}$ resides in the remaining 8 positions. The transformation $\theta$ therefore acts within each $E_{8}$ factor and on both factors simultaneously. This is a particular instance of the $\mathbb{Z}_{2}$-triple construction described previously. Note that $\theta\left(\mathbf{a}_{i}^{\prime}\right)-\mathbf{a}_{i}^{\prime} \neq 0$ for either $i=2$ or $i=3$, but that in both cases the difference is a root vector of $E_{8} \times E_{8}$. 
We see that the CHL string, $\operatorname{Spin}(32) / \mathbb{Z}_{2}$ compactification without vector structure and the $\mathbb{Z}_{2}$-triple are indeed equivalent, as claimed. Our duality chain does not end here, but by proceeding straightforwardly, we would end up with non-standard coordinates on group-lattices for the theories that we encounter. To avoid possible confusion, let us perform a coordinate transformation on the group lattice of $E_{8} \times E_{8}$ so that the first $E_{8}$ ends up in the first 8 positions again, and the second in the second 8 . Furthermore, the coordinates are chosen such that,

$$
\begin{aligned}
\theta\left(u_{i}\right) & =-u_{i}, \quad i=5, \ldots, 12, \quad \theta\left(u_{i}\right)=u_{i}, \quad i \neq 5, \ldots, 12 \\
\mathbf{a}_{2}^{\prime} & =\left(\frac{1^{2}}{2}, 0^{4}, \frac{1^{4}}{2}, 0^{4}, \frac{1^{2}}{2}\right) \\
\mathbf{a}_{3}^{\prime} & =\left(\left(0, \frac{1}{2}\right)^{4},\left(-\frac{1}{2}, 0\right)^{4}\right) .
\end{aligned}
$$

We stress that we have not changed anything in the theory. It is easy to check that the coordinate transformation can be chosen so that it corresponds to a lattice symmetry, or equivalently, a gauge transformation. To emphasize this, we will continue using the symbols $\theta$ and $\mathbf{a}_{i}^{\prime}$ since we are working with the same theory as before.

Let us continue our study of the $\mathbb{Z}_{2}$-triple in $E_{8} \times E_{8}$ on a 3-torus with radii $\left(R_{1} / 2, R_{2}^{\prime}, R_{3}^{\prime}\right)$ and gauge group $\left(\operatorname{Spin}(8)_{2}\right)^{2}$. We compactify the theory on a fourth circle with radius $R_{4}$ and turn on a holonomy parametrised by,

$$
\mathbf{a}_{4}=\left(1,0^{14},-1\right)
$$

This breaks the gauge group to $\left(\operatorname{Spin}(4)_{2}\right)^{4}=\left(S U(2)_{2}\right)^{8}$. We dualise along the 4-direction to a $\operatorname{Spin}(32) / \mathbb{Z}_{2}$ theory on a fourth circle with radius $R_{4}^{\prime}=$ $\alpha^{\prime} / 2 R_{4}$ and holonomy parametrised by,

$$
\mathbf{a}_{4}^{\prime}=\left(\left(0,-\frac{1}{2}\right)^{2},\left(0, \frac{1}{2}\right)^{2},\left(0,-\frac{1}{2}\right)^{2},\left(0, \frac{1}{2}\right)^{2}\right) .
$$

We have discussed the physical interpretation of this theory briefly in the section on gauge bundles over the 4-torus. The transformation $\theta$ does not leave any of the $\mathbf{a}_{i}^{\prime}$ invariant, but now $\theta\left(\mathbf{a}_{i}^{\prime}\right)-\mathbf{a}_{i}^{\prime}$ are roots for all $i$. This theory is 
therefore not a compactification without vector structure. Instead, we should interpret this theory as a particular case of a quadruple of $\operatorname{Spin}(32) / \mathbb{Z}_{2}$ on a 4-torus.

To arrive at the final theory on this chain, we compactify on a fifth circle with radius $R_{5}$, and turn on a holonomy parametrised by,

$$
\mathbf{a}_{5}=\left(\frac{1}{2}^{2},\left(-\frac{1}{2}\right)^{2}, 0^{8}, \frac{1}{2}^{2},\left(-\frac{1}{2}\right)^{2}\right) .
$$

The resulting gauge group is $U(1)^{8}$. We dualize along the 5 direction to obtain an $E_{8} \times E_{8}$ theory where the fifth circle has radius $R_{5}^{\prime}=\alpha^{\prime} / 2 R_{5}$ and holonomy,

$$
\mathbf{a}_{5}^{\prime}=\left(0^{2},-1,0,1,0^{11}\right) .
$$

The first $E_{8}$ factor is in the positions $i=5, \ldots, 12$, while the second occupies the positions $i=1, \ldots, 4,13, \ldots, 16$. None of the $\mathbf{a}_{i}$ are invariant. The transformation $\theta$ inverts all coordinates of one of the $E_{8}$ 's; this compactification should therefore be interpreted as a quintuple embedded in one of the $E_{8}$ gauge factors.

This chain of 5 theories shows again that the list of toroidal compactifications in string theory is much shorter than the list of gauge theory compactifications. Among the gauge theory compactifications that can be implemented consistently in string theory, there are many equivalences. Various choices of bundles just correspond to different limits in the string moduli space. It also shows that the topology of the gauge bundle, although entering crucially in our analysis, is a not a sharp notion to a string. For example, note that according to our preceeding analysis, certain compactifications of $\operatorname{Spin}(32) / \mathbb{Z}_{2}$ without vector structure are connected to compactifications with vector structure and another mechanism of rank reduction. As we will see later, this provides connections between seemingly different dual theories. In particular, between choices of bundles in configurations of D-branes on orientifolds. 
In the 5 dual descriptions obtained above, $\mathbf{a}_{i}$ and $\mathbf{a}_{i}^{\prime}$ are always of length $\sqrt{2}$. This is not the minimum value. As we remarked in the previous section, the minimal value for the $\mathbb{Z}_{2}$-theory is 1 . To reach this minimal value, we can deform the $\mathbf{a}_{i}^{\prime}$ by adding vectors in $\mathbb{R}_{16}$ which are left invariant by $\theta$. By doing so, we can flow to a 'canonical' point in the moduli space. At such a point, there is non-abelian gauge symmetry. We have listed the gauge symmetries appearing at these 'canonical points' in table 6 , where we have also included the Kaluza-Klein gauge bosons. We have also listed the Mikhailov lattices $\Gamma_{(10-n)}$ derived in [38]. The expression for $\Gamma_{(5)}$ is not found in [38], but is easily derived with our methods.

\begin{tabular}{|c|c|c||c|c|}
\hline Theory & bundle & $n$ & Symmetry group & $\begin{array}{c}\text { Mikhailov } \\
\text { lattice }\end{array}$ \\
\hline$E_{8} \times E_{8}$ & CHL & 1 & $\left(E_{8}\right)_{2} \times U(1) \times U(1)$ & $E_{8} \oplus \Gamma_{1,1}$ \\
$\operatorname{Spin}(32) / \mathbb{Z}_{2}$ & NVS & 2 & $\left(S p(8) / \mathbb{Z}_{2}\right) \times U(1)^{2} \times U(1)^{2}$ & $D_{8} \oplus \Gamma_{2,2}$ \\
$E_{8} \times E_{8}$ & triple & 3 & $F_{4} \times F_{4} \times U(1)^{3} \times U(1)^{3}$ & $D_{4} \oplus D_{4} \oplus \Gamma_{3,3}$ \\
$S p i n(32) / \mathbb{Z}_{2}$ & quadruple & 4 & $S p i n(17) \times U(1)^{4} \times U(1)^{4}$ & $D_{8}^{*}(2) \oplus \Gamma_{4,4}$ \\
$E_{8} \times E_{8}$ & quintuple & 5 & $E_{8} \times U(1)^{5} \times U(1)^{5}$ & $E_{8}(2) \oplus \Gamma_{5,5}$ \\
\hline
\end{tabular}

Table 6: Asymmetric $\mathbb{Z}_{2}$-orbifolds of heterotic theories on an $n$-torus.

On the canonical points in the moduli space, the connection between the groups and in particular their lattices, and the lattices of Mikhailov is clear: The lattices are related to the short roots of the symmetry groups. This provide a physical link to aspects of Mikhailov's mathematical constructions. Miklailov argues that his lattices are also useful for understanding aspects of dual theories. This point will be revisited later in our paper.

\section{More quintuples}

Let us now set aside compactifications of the CHL string, and turn to other theories that admit dual realizations. The endpoint of our $\mathbb{Z}_{2}$-chain was given by the $E_{8} \times E_{8}$ theory with a quintuple in one $E_{8}$. Actually, with an $E_{8}$ theory on a 5 -torus and holonomies $\mathbf{a}_{2}^{\prime}, \mathbf{a}_{3}^{\prime}, \mathbf{a}_{4}^{\prime}$ and $\mathbf{a}_{5}^{\prime}$ given by eqs. (36), 
(37), (39) and (41), it is possible to have quintuples in both $E_{8}$ factors. For this purpose, we construct the asymmetric orbifold obtained by shifting over $\pi R_{1}$ combined with the complete reflection on the gauge group lattice:

$$
\theta\left(u_{i}\right)=-u_{i}
$$

Only discrete gauge symmetries survive in this construction. All continuous gauge symmetry is broken.

This $E_{8} \times E_{8}$ theory on a 5 -torus with radii $\left(R_{1} / 2, R_{2}, R_{3}, R_{4}, R_{5}\right)$ with quintuples in each $E_{8}$ may be easily dualized in either the $2,3,4$ or 5 directions, since these are all equivalent. For ease of notation, we will dualize in the 5 direction with,

$$
R_{5} \rightarrow \frac{\alpha^{\prime}}{2 R_{5}} \quad \mathbf{a}_{5}^{\prime} \rightarrow \mathbf{a}_{5}
$$

and where $\mathbf{a}_{5}$ is given in eq. (40). We have obtained a $\operatorname{Spin}(32) / \mathbb{Z}_{2}$ theory on the 5-torus with completely broken gauge group. Checking the action of $\theta$ on the $\mathbf{a}_{i}^{\prime}$ and $\mathbf{a}_{5}$ reveals that this is a case of a quintuple in $\operatorname{Spin}(32) / \mathbb{Z}_{2}$. Notice that this duality connects two intrinsically five dimensional theories: decompactification of dimensions on either side of the duality would restore some gauge symmetry.

\section{The $\mathbb{Z}_{4}$ chain}

Take the heterotic $E_{8} \times E_{8}$ theory with a $\mathbb{Z}_{4}$-triple on a 3 -torus. We use standard coordinates on $E_{8} \times E_{8}$ so that the first $E_{8}$ is in the first 8 positions, and the second $E_{8}$ in the remaining 8. We turn on holonomies $\mathbf{a}_{2}$ and $\mathbf{a}_{3}$. To obtain the $\mathbb{Z}_{4}$-triple, we divide by a shift over $\pi R_{1} / 2$ combined with a $\theta$ 
of order 4. In a special case, the expressions for $\theta$ and $\mathbf{a}_{i}$ can be taken to be,

$$
\begin{aligned}
\theta\left(u_{i}, u_{i+1}, u_{i+2}\right) & =\left(-u_{i+2},-u_{i+1}, u_{i}\right), \quad i=1,9 \\
\theta\left(u_{i}, u_{i+1}, u_{i+2}, u_{i+3}, u_{i+4}\right) & =\left(-u_{i+4},-u_{i+3},-u_{i+2},-u_{i+1}, u_{i}\right), \quad i=4,13 \\
\mathbf{a}_{2} & =\left(\frac{1^{3}}{2}, 0^{5},\left(-\frac{1}{2}\right)^{3}, 0^{5}\right), \\
\mathbf{a}_{3} & =\left(\frac{1}{2}, \frac{1}{4}, 0, \frac{1}{2},\left(\frac{1}{4}\right)^{3}, 0, \frac{1}{2}, \frac{1}{4}, 0, \frac{1}{2},\left(\frac{1}{4}\right)^{3}, 0\right) .
\end{aligned}
$$

To clarify these formulae a little, let us elaborate somewhat on the steps in the triple construction. Starting with a torus without holonomies, turning on $\mathbf{a}_{2}$ will break $E_{8} \times E_{8}$ to $\left(\operatorname{Spin}(6)^{2} \times \operatorname{Spin}(10)^{2}\right) / \mathbb{Z}_{4}$. The $\mathbb{Z}_{4}$ is generated by the products of the generators of the $\mathbb{Z}_{4}$ centers of $\operatorname{Spin}(6)=S U(4)$ and $\operatorname{Spin}(10)$. Therefore, it acts diagonally on all factors. The reader may anticipate that $\left(\operatorname{Spin}(6)^{2} \times \operatorname{Spin}(10)^{2}\right) / \mathbb{Z}_{4}$ is a group that can also be obtained by compactifying $\operatorname{Spin}(32) / \mathbb{Z}_{2}$ with two holonomies. The remaining holonomies embed twisted boundary conditions: $\mathbf{a}_{3}$ breaks $\operatorname{Spin}(6)$ to $U(1)^{3}$ and $\operatorname{Spin}(10)$ to $S U(3) \times U(1)^{3}$. Finally, the $\theta$ quotients eliminate all $U(1)$ 's and mod $S U(3)$ by its outer automorphism to $S U(2)$ which is the surviving gauge group. The holonomies defined above are equivalent to the ones described abstractly in the previous section. The theory is at what we have called a canonical point in the moduli space.

We will not dualize this theory directly, but instead make a slight detour to stress some subtle points. We start by compactifying on a fourth circle, with radius $R_{4}$, without turning on a holonomy on this circle. Instead we will perform an $S L(4, \mathbb{Z})$ transformation on the 4-torus:

$$
x_{4} \rightarrow x_{4}-2 x_{2} \quad x_{i} \rightarrow x_{i} \quad i \neq 4 .
$$

In this way, we obtain a theory with identical spectrum but with holonomies parametrised by $\theta, \mathbf{a}_{2}, \mathbf{a}_{3}$ and a holonomy around the fourth circle given by $\mathbf{a}_{4}=2 \mathbf{a}_{2}$. Although none of the $\mathbf{a}_{i}$ is invariant under $\theta$, this theory on the 
4 -torus can be decompactified to one on the 3-torus by decompactifying in the $x_{4}-2 x_{2}$ direction.

We now turn on a $B$-field, which has as its only non-zero components $B_{24}=-B_{42}=3 / 2$. The purpose of this, as may be verified with the aid of the formulae given in appendix $B$, is so we can use lattice symmetries to re-express this as an equivalent theory with $B_{i j}=0$ and holonomies parametrised by $\theta, \mathbf{a}_{2}, \mathbf{a}_{3}$. The holonomy around the fourth circle is now given by,

$$
\tilde{\mathbf{a}}_{4}=\left(0^{7}, 1,0^{7},-1\right) \text {. }
$$

We have arrived at the theory we wish to dualize. Doing so gives a theory where the fourth radius is $R_{4}^{\prime}=\alpha^{\prime} / 2 R_{4}$. There is a holonomy parametrised by $\mathbf{a}_{4}^{\prime}$ around the 4 direction, and there is a non-zero $B$-field. The non-zero components are given by,

$$
\mathbf{a}_{4}^{\prime}=\left(0^{8},-\frac{1}{2}, \frac{1^{2}}{2},-\frac{1}{2}, \frac{1}{2}^{4}\right) \quad B_{24}=-B_{42}=\frac{1}{4} .
$$

The remaining moduli are given by $\mathbf{a}_{2}$ and $\mathbf{a}_{3}$.

We may ignore the $B$-field, which does play a role in the dualities, but not in the gauge field interpretation. ${ }^{8}$ We have found a $\operatorname{Spin}(32) / \mathbb{Z}_{2}$ theory. It is not too hard to verify that $\theta\left(\mathbf{a}_{3}\right)-\mathbf{a}_{3}$ is on the spin lattice of $\operatorname{Spin}(32) / \mathbb{Z}_{2}$, indicating that we are dealing with a compactification without vector structure. The rank reduction is, however, not equal to 8 but to 14. This is a realization of a quadruple without vector structure. All the Chern-Simons invariants that can be defined over sub-three-tori of the four-torus are integer.

The existence of a $\operatorname{Spin}(32) / \mathbb{Z}_{2}$ description of this theory implies the existence of a type I theory on the 4-torus with the same bundle. By Tdualities, this translates into orientifolds of type II theories to be described in section 3 .

\footnotetext{
${ }^{8}$ Besides, we can always deform the $B$-field away.
} 


\section{$\mathbb{Z}_{2} \times \mathbb{Z}_{2}$ asymmetric orbifolds}

Consider an $E_{8} \times E_{8}$ theory on a 4-torus with holonomies parametrised by,

$$
\begin{aligned}
& \mathbf{a}_{3}=\left(1,0^{14},-1\right), \\
& \mathbf{a}_{4}=\left(0^{4}, \frac{1^{4}}{2},\left(-\frac{1}{2}\right)^{4}, 0^{4}\right) .
\end{aligned}
$$

We construct a $\mathbb{Z}_{2}$-triple in this theory by dividing this theory by a $\pi R_{1}$ shift over the first circle combined with

$$
\theta_{1}: \quad\left(u_{1}, \ldots, u_{8}, u_{9}, \ldots u_{16}\right) \rightarrow-\left(u_{8}, \ldots, u_{1}, u_{16}, \ldots u_{9}\right)
$$

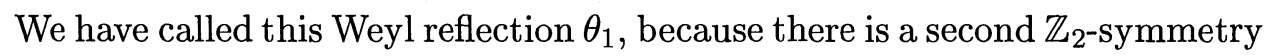
that we wish to use in an orbifold construction. Consider

$$
\theta_{2}: \quad\left(u_{1}, \ldots, u_{16}\right) \rightarrow-\left(u_{16}, \ldots, u_{1}\right)
$$

Note that $\mathbf{a}_{3}$ and $\mathbf{a}_{4}$ are invariant under $\theta_{2}$, which is an outer automorphism. Hence it is consistent to divide this theory by a $\mathbb{Z}_{2}$-shift over $\pi R_{2}$ combined with $\theta_{2}$. The resulting theory combines the CHL construction with the $\mathbb{Z}_{2^{-}}$ triple. The rank of the gauge group is reduced by 12 .

We may dualize, for example, in the 3 direction to obtain a $\operatorname{Spin}(32) / \mathbb{Z}_{2}$ theory with $\mathbf{a}_{3}$ replaced by:

$$
\mathbf{a}_{3}^{\prime}=\left(\left(-\frac{1}{2}\right)^{2}, \frac{1}{2}^{2},\left(-\frac{1}{2}\right)^{2}, \frac{1}{2}^{2}, 0^{8}\right) .
$$

Note that $\mathbf{a}_{3}^{\prime}$ is invariant under $\theta_{1}$, while $\theta_{2}\left(\mathbf{a}_{3}^{\prime}\right)-\mathbf{a}_{3}^{\prime}$ lies on the spin weight lattice. On the other hand $\mathbf{a}_{4}$ is invariant under $\theta_{2}$, while $\theta_{1}\left(\mathbf{a}_{4}\right)-\mathbf{a}_{4}$ lies on the spin weight lattice. The resulting theory is interpreted as a theory without vector structure, which has $\tilde{w}_{2}^{2} \neq 0$. Notice that we have now encountered all three types of bundles for $\operatorname{Spin}(32) / \mathbb{Z}_{2}$ on $T^{4}$ that we met in section 2.1.4. They appear for respectively for $G=\mathbb{Z}_{2}, G=\mathbb{Z}_{4}$, and $G=\mathbb{Z}_{2} \times \mathbb{Z}_{2}$. 


\subsubsection{Degeneration limits: connections to other models}

We have constructed moduli spaces for a number of asymmetric orbifold theories. These moduli space are non-compact and the infinities correspond to decompactification limits. The moduli space of the $D=9 \mathrm{CHL}$ string is [38],

$$
O\left(\Gamma_{1,1} \oplus E_{8}\right) \backslash O(9,1) /(O(9) \times O(1)) .
$$

We have omitted a factor $\mathbb{R}^{+}$corresponding to the expectation value of the dilaton $\phi$. Moving to the end points of $\mathbb{R}^{+}$corresponds to the weak and strong-coupling limits of the theory. Our preceeding discussion has been limited to weak coupling, where $e^{\phi} \rightarrow 0$. There are various strong coupling limits which depend on how the string scale is treated as $e^{\phi} \rightarrow \infty$. At least in certain regions of the moduli space, there are $\mathrm{M}$ and $\mathrm{F}$ theory dual descriptions of the strongly-coupled heterotic string. We shall discuss some of these duals in section four.

The remaining infinities correspond to decompactification limits. In [38], it is shown that there exists only one light-like vector in $\Gamma_{1,1} \oplus E_{8}$, modulo the symmetry group. One of the elements of the symmetry groups inverts this vector. Therefore, there are two directions in which one can decompactifyroughly by taking $R \rightarrow \infty$ or $R \rightarrow 0$. These two limits appear to correspond to physically distinct theories, because the orbifold projection involves a shift over the compactification circle, and therefore explicitly involves $R$.

In the standard picture [2] that we have also used so far, the winding numbers are fractionalized. The masses of excitations from the twisted sector are then offset by a contribution that is linear in $R$ for large $R$. As $R \rightarrow$ $\infty$, the states in the twisted sector become infinitely heavy, and therefore decouple. In this limit, the shift becomes irrelvant and so we expect to recover the conventional ten-dimensional $E_{8} \times E_{8}$ heterotic string.

On the other hand, in the limit $R \rightarrow 0$, there are states in the twisted sector that become massless. These massless states from the twisted sector 
transform in the adjoint of $E_{8}$, and as $R$ goes to zero, these enhance the gauge group to $E_{8} \times E_{8}$ with one $E_{8}$ coming from the twisted sector and one from the untwisted sector. This limit is therefore also smooth.

A perhaps more apropriate way to describe this is by T-dualizing, taking $R \rightarrow \alpha^{\prime} / R$. This also exchanges momenta and winding numbers so that now we have a theory with fractionalized momenta and integer winding numbers. Fractionalized momenta are common in theories with background gauge fields. This may seem unusual for the CHL theory, but is an appropriate way to think about compactifications without vector structure, triples, quadruples and quintuples. Indeed, since the CHL outer automorphism is an orbifold action, it is a discrete gauge symmetry. Associated to this discrete gauge symmetry is a discrete gauge-field modulus which is fractionalizing the momenta.

At least in one limit, the asymmetric orbifold, which is often deemed nongeometric, has a natural interpretation in terms of a gauge bundle, which is a geometric concept. It is an interesting observation that asymmetric orbifolds can be interpreted in terms of bundles. Combining, such bundles with symmetric orbifolds may offer geometric interpretations for at least a particular class of asymmetric orbifolds.

Having settled this subtlety, the rest of our discussion is parallel to the last section of [38]. Large volume limits can be identified from our discussion of dualities. The 8-dimensional CHL string has various decompactification limits in which a two-torus becomes large. One of these corresponds to the $\operatorname{Spin}(32) / \mathbb{Z}_{2}$ compactification without vector structure, and one to the CHL string. The 7-dimensional CHL string has various limits which we have identified as the CHL string, $\operatorname{Spin}(32) / \mathbb{Z}_{2}$ without vector structure and the $E_{8} \times E_{8} \mathbb{Z}_{2}$-triple. This is in disagreement with [38] where two $\operatorname{Spin}(32)$ and one $E_{8} \times E_{8}$ degenerations were found. That analysis, however, is far less concerned with identifying the theories that appear in these limits. The 6-dimensional CHL string brings us one new limit where a 4-torus becomes 
large, which is identified as a quadruple, which has vector structure. Another new limit is found in 5-dimensions in terms of a quintuple in one $E_{8}$. Also, in the cases of $\mathbb{Z}_{4}$ and $\mathbb{Z}_{2} \times \mathbb{Z}_{2}$, we have identified the various limits.

The duality chains of this section connect a diverse set of theories encountered later in this paper. Note in particular the $\operatorname{Spin}(32) / \mathbb{Z}_{2}$ theories in various chains. By $\mathrm{S}$ and $\mathrm{T}$-dualities these will translate into type I theories and type II orientifolds, which make their appearance in section 3.

We will make a brief excursion to four dimensions where it is believed that the heterotic string theory is S-dual. In [38], this translates into a property of the Mikhailov lattice: namely, that it is isomorphic to its dual lattice up to a rescaling by a factor of $\sqrt{2}$. One way to heuristically understand the factor of $\sqrt{2}$ is that it is related to the possible appearance of non-simplylaced groups in CHL theories. For non-simply-laced groups $G$, the gauge group in the S-dual theory is given by the dual group $G_{D}$. The roots of $G_{D}$ are the coroots of $G$ up to a suitable rescaling [40], while the weight lattice of $G_{D}$ is the coweight lattice of $G$, also rescaled. The rescaling is precisely the factor of $\sqrt{2}$ for the 2-laced theories appearing in CHL compactifications.

By analogy the asymmetric $\mathbb{Z}_{m}$-orbifolds with $m=3,4,5,6$ should have lattices for their 4-dimensional theories that are isomorphic to their dual lattices up to rescaling by a factor of $\sqrt{m}$. The reader may verify that using the lattices $\Lambda$ from table 5 , the lattices $\Gamma_{3,3}(m) \oplus \Lambda$ indeed have this property. This is again closely related to the fact that the $F_{4}, G_{2}$ and $A_{1}$ at level 4 are their own dual groups up to rotations [40]. It is amusing to see structure in a theory compactified on a 6 -torus reflected in a similar theory compactified on the 3 -torus.

Also the table 6 suggests that the 7-dimensional theory has special status because the groups and lattices listed there for $(7+d)$ are the duals of those in $(7-d)$. This is somehow related to the $\mathbb{Z}_{2}$ nature of the duality operation, and to the fact that $3=6 / 2$, but a more precise understanding of this relation is in order. 
We also wish to briefly comment on other heterotic theories appearing in the literature. In particular we note that $\mathbb{Z}_{2}, \mathbb{Z}_{3}, \mathbb{Z}_{4} \mathbb{Z}_{5}, \mathbb{Z}_{6}$ and $\mathbb{Z}_{2} \times \mathbb{Z}_{2}$ asymmetric orbifolds in dimensions less than 7 do appear in $[41,42]$. The models in these papers are similar to the construction of [14], and originate in exploiting symmetries of $\mathrm{K} 3$. That heterotic duals should exist is obvious from the constructions, but an explanation for their existence has been lacking. The gauge theory based analysis presented here fills that gap. It also makes clear that these models can be traced back to constructions in higher dimensions. This again presents new challenges for finding dual descriptions which we take up in the following sections.

\subsubsection{A strong coupling description of the $\mathbb{Z}_{2}$ triple}

Let us conclude our discussion of heterotic string theory by pointing out an intriguing relation between the $\mathbb{Z}_{2}$ triple and a discrete 3 -form flux appearing in its strong coupling description.

The type I string on $T^{2}$ without vector structure can be viewed as an orientifold of type IIB with a half-integral NS-NS $B$-field flux through the $T^{2}[43,44]$. It is natural to ask whether the strong coupling Horava-Witten description of the $E_{8} \times E_{8}$ heterotic string might permit a similar discrete flux. Consider $\mathrm{M}$ theory on $S^{1} / \mathbb{Z}_{2} \times T^{3}$. The $\mathbb{Z}_{2}$ action projects out the $\mathrm{M}$ theory 3 -form $C$-field. After all, there are no membranes in heterotic string theory. The component of the $C$-field with a leg on $S^{1} / \mathbb{Z}_{2}$, however, survives projection and couples to the perturbative heterotic string.

It is natural to ask whether we can turn on a half-integral $C$-field on $T^{3}$ and then quotient by $\mathbb{Z}_{2}$. It is not clear that such a compactification is consistent but the following chain of dualities suggests that it exists and is an alternate description of the $\mathbb{Z}_{2}$-triple. Let us perform a $9-11$ flip and reduce from $\mathrm{M}$ theory to string theory on a circle of the $T^{3}$. This gives an orientifold of IIA on $S^{1} / \mathbb{Z}_{2} \times T^{2}$ with a half-integral $B$-field through $T^{2}$. A further T-duality on $S^{1} / \mathbb{Z}_{2}$ turns this compactification into type I on 
$S^{1} \times T^{2}$ with a half-integral $B$-field. As we recalled above, this is just type I with no vector structure which is in the same moduli space as the $\mathbb{Z}_{2}$-triple. This suggests an intimate connection between background 3-form fluxes and non-trivial Chern-Simons invariants for the $E_{8} \times E_{8}$ gauge bundle.

\section{Orientifolds}

\subsection{Background and definitions}

We begin our discussion of orientifolds with some background and some words on our notation. We use $\mathcal{I}_{9-p}$ to denote the sign flip of the last $9-p$ spatial coordinates of $\mathbb{R}^{10}$,

$$
\left(x_{0}, \ldots, x_{p}, x_{p+1}, \ldots, x_{9}\right) \mapsto\left(x_{0}, \ldots, x_{p},-x_{p+1}, \ldots,-x_{9}\right) .
$$

Type II string theory on $\mathbb{R}^{10}$ is invariant under the action of $\mathcal{I}_{9-p}$ when combined with world-sheet orientation reversal $\Omega$, where $p$ is even for type IIA and odd for type IIB. As is standard, we use $(-1)^{F_{L}}$ to denote the symmetry that flips the sign of all R-NS and RR states. It is not hard to check that,

$$
\mathcal{I}_{9-p} \Omega,
$$

is an involution, i.e., squares to the identity, for $p=0,1,4,5,8,9$ while,

$$
\mathcal{I}_{9-p}(-1)^{F_{L}} \Omega
$$

is an involution for $p=7,6,3,2$. We can then consider an orbifold of Type II string theory by the $\mathbb{Z}_{2}$ symmetry group generated by this involution. This is called a Type II orientifold on $\mathbb{R}^{10} / \mathcal{I}_{9-p}$, or just $\mathbb{R}^{10} / \mathbb{Z}_{2}$ when there is no room for confusion. The $\mathbb{Z}_{2}$ fixed plane $x_{p+1}=\cdots=x_{9}=0$ is called an orientifold $p$-plane, or $O p$ plane for short. We can also extend this construction to the case where $\mathbb{R}^{10}$ is replaced by a non-trivial ten-manifold $M^{10}$ with an involution $\mathcal{I}$, as we will do in the case of $T^{9-p} \times \mathbb{R}^{p+1}$ where $\mathcal{I}$ acts by inversion on the $(9-p)$ coordinates of $T^{9-p}$. 
There are two kinds of orientifold planes, $O p^{-}$and $O p^{+}$, which are distinguished by the sign of the closed string $\mathbb{R} \mathbb{P}^{2}$ diagram surrounding the plane $-O p^{+}$has an extra $(-1)$ factor when compared to $O p^{-}$. When $N$ $\mathrm{D} p$-branes are placed on top of $O p^{-}$(resp. $\left.O p^{+}\right)$, they support an $S O(N)$ (resp. $U S p(N)=S p(N / 2)$ ) gauge group, where we count the number of Dbranes on the double cover. An $O p$ plane carries D $p$-brane charge, and the two kinds of $O p$ planes are also distinguished by the sign of this charge$O p^{ \pm}$carries D $p$-brane charge $\pm 2^{p-5}$ when counted on the double cover. The superscript ${ }^{+}$or $^{-}$in the name of the plane has its origin here.

An $O p^{-}$plane together with an even number of $\mathrm{D} p$-branes is quite different in character from an $O p^{-}$plane together with an odd number of $\mathrm{D} p$-branes. We distinguish these two cases by using the notation $O p^{{ }^{\prime}}$ for an $O p^{-}$plane with a single $\mathrm{D} p$-brane stuck to it. It has been shown that $O p^{-^{\prime}}$ has a non-trivial $\mathbb{Z}_{2}$ flux associated with the RR $(6-p)$-form $G_{6-p}$, while the flux is trivial for $\mathrm{Op}^{-}$. It has also been shown that there are two kinds of $\mathrm{Op}^{+}$planes distinguished by the same flux; we denote the trivial one by $\mathrm{Op}^{+}$and the non-trivial one by $\mathrm{Op}^{{ }^{+}}$. In total, there are four kinds of orientifold planes:

$$
O p^{-}, O p^{-^{\prime}}, O p^{+}, O p^{+^{\prime}}
$$

These four kinds of orientifold planes-especially the planes $O p^{-^{\prime}}$ and $O p^{+^{\prime}}$ with non-trivial $\mathbb{Z}_{2}$ flux-have been identified for the cases $p=5,4,3,2,1,0$ $[15,45-50]$. We refer to these references for a fuller discussion of the $\mathbb{Z}_{2}$ flux associated with $G_{6-p}$.

\subsubsection{The closed string perturbation expansion}

As mentioned above, $O p^{+}$and $O p^{+^{\prime}}$ have an extra (-1) sign when compared to $O p^{-}$and $O p^{{ }^{\prime}}$ for the fundamental string $\mathbb{R P}^{2}$ diagram. The meaning of an ' $\mathbb{R P}^{2}$ surrounding the plane' is clear only if the transverse dimension $(9-p)$ is greater or equal to 3 . We can actually make this statement more precise so that it also applies to the cases $(9-p)=1,2$. We note that the phase of the 
closed string diagram is given by the $B$-field, and therefore it is natural to use equivariant cohomology to determine the allowed $B$-field configurations. Let us consider the closed string perturbation expansion for a type II orientifold on $M / \mathcal{I}$ following [17]. The world-sheet is an orientable Riemann surface $\Sigma$ with orientation reversing freely-acting involution $\mathcal{I}_{\Sigma}$. Note that the quotient $\Sigma / \mathcal{I}_{\Sigma}$ is a smooth unorientable surface if $\Sigma$ is connected. However, it is smooth and orientable but not oriented when $\Sigma$ consists of two identical components exchanged by $\mathcal{I}_{\Sigma}$. The world-sheet path-integral is over maps $\phi: \Sigma \rightarrow M$ which commute with the involution,

$$
\mathcal{I} \circ \phi=\phi \circ \mathcal{I}_{\Sigma}
$$

We would like to assign a phase factor in a way that is consistent with all physical consistency conditions, such as factorization etc. This can be accomplished in the following way. Let us fix an element of the equivariant cohomology group,

$$
y \in H_{\mathbb{Z}_{2}}^{2}\left(M, \mathbb{Z}_{2}\right) .
$$

This means choosing an element of $H^{2}\left(M_{\mathbb{Z}_{2}}, \mathbb{Z}_{2}\right)=H^{2}\left(S^{N} \times_{\mathbb{Z}_{2}} M, \mathbb{Z}_{2}\right)$ for sufficiently large $N$, where $\mathbb{Z}_{2}$ acts on $S^{N}$ via the antipodal map and on $M$ by $\mathcal{I}{ }^{9}$ The map $\phi$, obeying the condition (60), induces a map $\hat{\phi}: \Sigma_{\mathbb{Z}_{2}} \rightarrow M_{\mathbb{Z}_{2}}$. Therefore the element $y \in H^{2}\left(M_{\mathbb{Z}_{2}}, \mathbb{Z}_{2}\right)$ can be pulled back to an element $\hat{\phi}^{*} y \in H^{2}\left(\Sigma_{\mathbb{Z}_{2}}, \mathbb{Z}_{2}\right)$. We denote this element simply by

$$
\phi^{*} y \in H_{\mathbb{Z}_{2}}^{2}\left(\Sigma, \mathbb{Z}_{2}\right) .
$$

\footnotetext{
${ }^{9}$ For a discussion of equivariant cohomology, see appendix D, and section 4.4. Here, we provide a brief summary of some basic definitions. The equivariant cohomology $H_{G}^{*}(X, R)$, with $G$ a group acting on a space $X$ and $R$ a coefficient ring, is defined to be the ordinary cohomology $H^{*}\left(M_{G}, R\right)$ where $M_{G}$ is the fibre product

$$
M_{G}=E G \times_{G} M:=(E G \times M) / G .
$$

Here $E G$ is the universal $G$-bundle over the classifying space $B G$. In the context of our orientifold discussion, $G=\mathbb{Z}_{2}=\{1, \mathcal{I}\}$ for $X=M$ and $R=\mathbb{Z}_{2}$.

For $G=\mathbb{Z}_{2}$, we can take $E G=S^{N}$ so that $B G=\mathbb{R P}^{N}$ with $N \rightarrow \infty$ strictly speaking. However, it is enough to take large but finite $N$ for most of the practical purposes. If $G$ acts on $X$ freely (as is the case for $X=\Sigma$ and $G=\left\{1, \mathcal{I}_{\Sigma}\right\}$ ), then $H_{G}^{*}(X, R)=H^{*}(X / G, R)$ since $E G$ is contractible.
} 
Since $\mathcal{I}_{\Sigma}$ acts on $\Sigma$ freely, $\phi^{*} y$ can be viewed as an element of $H^{2}\left(\Sigma / \mathcal{I}_{\Sigma}, \mathbb{Z}_{2}\right)$ which can be integrated over $\Sigma / \mathcal{I}_{\Sigma}$ :

$$
\left\langle\Sigma / \mathcal{I}_{\Sigma}, \phi^{*} y\right\rangle:=\int_{\Sigma / \mathcal{I}_{\Sigma}} \phi^{*} y \in \mathbb{Z}_{2} .
$$

We can then assign the following sign factor to the world-sheet path-integral,

$$
(-1)^{\left\langle\Sigma / \mathcal{I}_{\Sigma}, \phi^{*} y\right\rangle}
$$

Now, we can make precise the meaning of the sign of the $\mathbb{R P}^{2}$ diagram. Suppose $\mathcal{I}$ acting on $M$ has a fixed point $P$. The constant map $\phi_{0}$ from $S^{2} \rightarrow P$ trivially obeys condition (60). The map $\hat{\phi}_{0}: S_{\mathbb{Z}_{2}}^{2} \rightarrow M_{\mathbb{Z}_{2}}$ then collapses to the identity map $\mathbb{R P}^{N} \rightarrow \mathbb{R P}^{N}$ and the integral $\left\langle\mathbb{R P}^{2}, \phi_{0}^{*} y\right\rangle$ is simply evaluating $y$ on the 2 -cycle $S^{2} \times_{\mathbb{Z}_{2}}\{P\} \cong \mathbb{R P}^{2}$ of $M_{\mathbb{Z}_{2}}=S^{N} \times_{\mathbb{Z}_{2}} M$. This definition agrees with the standard one when $\mathcal{I}$ acts around $P$ by the action $\mathcal{I}_{9-p}$ with $(9-p) \geq 3$. To see this, first consider the space $M^{\circ}$ obtained from $M$ by deleting all the $\mathbb{Z}_{2}$ fixed points. By restriction, $y \in H_{\mathbb{Z}_{2}}^{2}\left(M, \mathbb{Z}_{2}\right)$ yields an element $y^{\circ} \in H_{\mathbb{Z}_{2}}^{2}\left(M^{\circ}, \mathbb{Z}_{2}\right)$. Since $\mathbb{Z}_{2}$ acts on $M^{\circ}$ freely, $y^{\circ}$ can be viewed as an element of $H^{2}\left(M^{\circ} / \mathcal{I}, \mathbb{Z}_{2}\right)$. We now note that the map $\hat{\phi}_{0}: S_{\mathbb{Z}_{2}}^{2} \rightarrow M_{\mathbb{Z}_{2}}$ can be continuously deformed to a map $\hat{\phi}_{\epsilon}$ where $\phi_{\epsilon}$ is a map of $S^{2}$ to a small $\mathbb{Z}_{2}$ invariant 2-sphere surrounding $P$ and lying in $M^{\circ}$. Or equivalently, a map from $\mathbb{R}^{2}$ to a small $\mathbb{R}^{2}$ surrounding $P$ and lying in $M^{\circ} / \mathcal{I}$. It is then clear that,

$$
\left\langle\mathbb{R P}^{2}, \phi_{0}^{*} y\right\rangle=\left\langle\mathbb{R P}^{2}, \phi_{\epsilon}^{*} y\right\rangle=\int_{\mathbb{R}^{2}} \phi_{\epsilon}^{*} y^{\circ}
$$

which is the standard meaning of the sign of the $\mathbb{R P}^{2}$ diagram surrounding $P$.

To summarize, the possible configurations of plus $\left(+\right.$ or $\left.+^{\prime}\right)$ versus minus $\left(-\right.$ or $\left.-^{\prime}\right)$ orientifolds can be classified from the closed string perspective by the group $H_{\mathbb{Z}_{2}}^{2}\left(M, \mathbb{Z}_{2}\right)$. As we shall see below, there can be further constraints. 


\subsubsection{Some pathologies}

We note that both $-^{\prime}$ and $+^{\prime}$ orientifolds are inconsistent for $p=9,8,7,6$ if we insist, as we shall, on backgrounds preserving sixteen supersymmetries. There are a number of ways to see this. For example, for $p=6$ we can probe the $O 6^{{ }^{\prime}}$ with a D2-brane. The theory on the probe is an $N=4 S p(1)$ gauge theory with a half-hypermultiplet in the fundamental representation. This three-dimensional gauge theory has a $\mathbb{Z}_{2}$ anomaly, and is therefore inconsistent. The only way to cancel such an anomaly is by including a Chern-Simons term which makes the gauge-field massive. However, ChernSimons terms can only be written down for $N \leq 3$ supersymmetry in $d=3$ [51]; $N=4$ supersymmetry does not allow a short massive vector multiplet. Rather, there are arguments given in [6] which suggest that $O 6^{{ }^{\prime}}$ planes require a non-zero cosmological constant, and so can only be found in massive type IIA supergravity. Similarly, if $\mathrm{O6}^{+^{\prime}}$ is distinguished from $O 6^{+}$by a flux associated with $G_{0}$, it is expected to be in a theory with a cosmological constant.

In our subsequent discussion, we shall classify - and -' configurations by using the fact that we can T-dualize these configurations to type I. The type I requirement that the $O(32)$ gauge bundle lift to a $\operatorname{Spin}(32) / \mathbb{Z}_{2}$ bundle with vanishing Chern-Simons invariant constrains the possible - and $-^{\prime}$ configurations. This actually gives an alternative and stronger argument for excluding $O 6^{{ }^{\prime}}$ in a $T^{3} / \mathbb{Z}_{2}$ orientifold. The details of the derivation are found in appendix C. This anomaly cancellation argument and the D2brane probe brane argument are not completely independent. Suppose that we naively construct the $T^{3} / \mathbb{Z}_{2}$ orientifold with $8 O 6^{-^{\prime}}$ planes. Somewhere, we should encounter an inconsistency. From the chain of dualities connecting this type IIA orientifold to the heterotic string on $T^{3}$, we find a hint of where to look. In the heterotic theory, we argued that obstructions to certain compactifications arise from states associated to winding strings. After S-duality, these correspond to winding D-strings in a type I compactification. The states on these winding strings transform non-trivially under a $\mathbb{Z}_{2}$ 
that should be divided out in the construction of the bundle. As a consequence, the phases of these states have a $\mathbb{Z}_{2}$ ambiguity, and the theory is not well-defined. Nevertheless, proceeding with this inconsistent theory and T-dualizing 3 directions leads to the $T^{3} / \mathbb{Z}_{2}$ orientifold with $8 O 6^{{ }^{\prime}}$ planes. In the process, the D-string becomes a D2-brane, and the $\mathbb{Z}_{2}$ ambiguity in the phases of winding states becomes the $\mathbb{Z}_{2}$ anomaly in the gauge theory on the probe brane. The anomaly therefore has multiple dual realizations.

\subsection{The classification}

Our discussion below focuses on type II orientifolds of $T^{9-p} \times \mathbb{R}^{D=p+1}$. We note that there are $2^{9-p} \mathbb{Z}_{2}$ fixed points, and the type of orientifold plane has to be specified at each fixed point. We use the notation

$$
\left\{-,-^{\prime},+,+^{\prime}\right\}
$$

for $O p^{-}, O p^{-^{\prime}}, O p^{+}$and $O p^{+^{\prime}}$, respectively. For example, the notation $\left(-,+,+,-^{\prime}\right)$ specifies a (possible) configuration in eight dimensions, or equivalently, a particular orientifold of $T^{2}$. For low enough dimensions, ordering is also required to completely specify the configuration.

In what follows we provide the complete list of possible orientifolds in dimensions $D:=p+1=10,9,8,7$. We also give the complete list of orientifolds involving only - and $-^{\prime}$ in dimensions $D=6,5$. In $D=6,5$ we do not attempt to completely classify configurations involving + and $+^{\prime}$ but we will comment on some of the new issues that arise. To see what kind of $\left\{+,+^{\prime}\right\}$ configurations are possible from the closed string point of view, we have included an explicit computation of the equivariant cohomology $H_{\mathbb{Z}_{2}}^{2}\left(T^{n}, \mathbb{Z}_{2}\right)$ for $n=1,2,3$ in appendix $\mathrm{D}$. The results are stated in the main text below.

\subsection{1 $D=10$}

The equivariant cohomology $H_{\mathbb{Z}_{2}}^{2}\left(\mathbb{R}^{10}, \mathbb{Z}_{2}\right)$ is the cohomology of the classifying space $B \mathbb{Z}_{2}=\mathbb{R P}^{\infty}$ which is $H^{2}\left(\mathbb{R P}^{\infty}, \mathbb{Z}_{2}\right)=\mathbb{Z}_{2}$. The two choices corre- 
spond to $\mathrm{O9}^{-}$and $\mathrm{O9}^{+} . \mathrm{O9}^{-}$has D9-brane charge -32 and can be cancelled by introducing $32 \mathrm{D} 9$-branes. Of course, this is the standard type I string on $\mathbb{R}^{10}$ which indeed preserves 16 supersymmetries. However, $\mathrm{O}^{+}$has positive D9-brane charge which is cancelled by introducing anti-D9-branes. Since $\mathrm{O}^{+}$and the anti-D9-branes preserve different supersymmetries, the system is not supersymmetric.

\subsection{2 $D=9$}

The equivariant cohomology has rank $2, H_{\mathbb{Z}_{2}}^{2}\left(T^{1}, \mathbb{Z}_{2}\right)=\mathbb{Z}_{2} \oplus \mathbb{Z}_{2}$. The two generators are non-zero on $\mathbb{R P}^{2}$ at the two fixed points of $S^{1}$ in the sense described above. The four elements correspond to

$$
(-,-),(+,-),(-,+),(+,+) .
$$

The configuration $(+,+)$ is not supersymmetric for the same reason that $\mathrm{O}^{+}$is not supersymmetric. The two configurations $(+,-)$and $(-,+)$are related by a diffeomorphism. Therefore in 9 dimensions, there are essentially 2 possible orientifold configurations. The first, $(-,-)$, has 32 D8-branes and is simply T-dual to type I compactified on a circle. This component also contains the $E_{8} \times E_{8}$ string [39].

The second component, $(-,+)$, has no net D-brane charge. Therefore no branes can be added while preserving supersymmetry so there is no enhanced gauge symmetry. This compactification is T-dual to type IIB on $S^{1} / \delta \Omega$ where $\delta$ is a half-shift along the circle and $\Omega$ is world-sheet parity. For a more detailed discussion, see $[17,52]$. Together with the CHL string, this gives a total of 3 distinct components in the moduli space of perturbative string compactifications.

\subsection{3 $D=8$}

The equivariant cohomology now has rank $4, H_{\mathbb{Z}_{2}}^{2}\left(T^{2}, \mathbb{Z}_{2}\right)=\left(\mathbb{Z}_{2}\right)^{4}$. The four generators are in one-to-one correspondence with the four $\mathbb{Z}_{2}$ fixed points- 
each has a non-trivial value on $\mathbb{R P}^{2}$ at the corrsponding fixed point and is trivial at the other three points. The possible orientifolds up to diffeomorphisms are

$$
(-,-,-,-), \quad(+,-,-,-), \quad(+,+,-,-) .
$$

The last is obtained from the sum of two generators. In addition, there are non-supersymmetric configurations.

The first component $(-,-,-,-)$ has $32 \mathrm{D} 7$-branes and is T-dual to the usual type I string on $T^{2}$. The second component $(-,-,-,+)$ is more interesting. The orientifold planes have total of -16 units of D7-brane charge and therefore require $16 \mathrm{D} 7$-branes. This orientifold is T-dual to type IIB on $T^{2}$ modded out by the world-sheet parity, $\Omega$, in the presence of a halfintegral background NS-NS $B$-field $[43,44]$. Although world-sheet parity sends $B \rightarrow-B$, a half-integral value of $B$ is permitted since $B$ takes values in a torus. This orientifold is equivalent to a compactification without vector structure [44]. By the T-duality argument of section 2.4.2, we know that this component containing type I without vector structure also contains the 8dimensional CHL string.

The third and final component $(-,-,+,+)$ is T-dual to the compactification of the 9-dimensional $(-,+)$ orientifold to eight dimensions. This has no D-branes and therefore no gauge symmetry.

\subsection{4 $D=7$}

The equivariant cohomology has rank $7, H_{\mathbb{Z}_{2}}^{2}\left(T^{3}, \mathbb{Z}_{2}\right)=\left(\mathbb{Z}_{2}\right)^{7}$. The seven generators $y_{1}, \ldots, y_{7}$ having the following property: let us use $P_{1}, \ldots, P_{8}$ to denote the eight $\mathbb{Z}_{2}$ fixed points of $T^{3}$. Then $y_{i}$ has a non-trivial value on both the $\mathbb{R P}^{2}$ surrounding $P_{i}$ and the one surrounding $P_{8}$, while it is trivial at the other six points. This shows that the allowed orientifolds are $(-8),\left(-6,+^{2}\right)$ and its permutations, and $\left(-{ }^{4},+^{4}\right)$ and its permutations. We exclude those cases with more + than - planes since they require antiD6-branes. That an odd number of $\mathrm{O6}^{+}$planes is not allowed can also be 
understood by an elementary argument. On $T^{3} / \mathbb{Z}_{2}$, we can enclose each $O 6$ plane with an $\mathbb{R P}^{2}$. The eight of them together correspond to a trivial cycle, and therefore the product of the eight $\mathbb{R P}^{2}$ diagrams must have sign +1 . Therefore, the number of $\mathrm{O6}^{+}$planes must be even.

The first case, $\left({ }^{8}\right)$, has 32 D6-branes. It is T-dual to the standard compactification of type I on $T^{3}$. The second case, $\left(-{ }^{6},+^{2}\right)$, requires 16 D6branes. All permutations are diffeomorphic to each other so there is essentially one configuration of this type. This is obtained from the $(-,-,-,+)$ orientifold on $T^{3} / \mathbb{Z}_{2}$ by compactification along another circle and T-duality on that circle.

The third case $\left(-{ }^{4},+^{4}\right)$ has no D6-branes and therefore no open strings. In this case, there is an interesting subtlety. The various permutations are not necessarily diffeomorphic to each other. There are essentially 2 distinct ways to place the orientifold planes at the vertices of the cube. The first can be characterized by placing the four - orientifold planes on a single face of the cube. The remaining four + planes are found at the remaining vertices. This is the configuration that follows from a dimensional reduction of the $(-,-,+,+)$ case. It is therefore automatically consistent. However, we could also consider the case where a single adjacent pair of + and - planes are interchanged. This gives a distinct configuration. For a fixed ordering of the $\mathbb{Z}_{2}$ fixed plane, let us denote these 2 cases by $(-,-,-,-,+,+,+,+)$ and by $(-,-,-,+,+,+,+,-)$. Both possibilities are allowed from the closed string point of view. Both are realized as elements of $H_{\mathbb{Z}_{2}}^{2}\left(T^{3}, \mathbb{Z}_{2}\right)$. This is easy to see because the equivariant cohomology is a group. Noting that all permutations of $(-,-,-,-,-,-,+,+)$ are realized as the elements of the group, we see that the first case is simply the sum of $(-,-,-,-,+,+,-,-)$ and $(-,-,-,-,-,-,+,+)$. The second possibility is the sum of $(-,-,-,+,+,-,-,-)$ and $(-,-,-,-,-,+,+,-)$. Thus, both are consistent configurations in perturbative closed string theory and they are distinct. Although the two are distinct configurations perturbatively, it is possible that they are equivalent non-perturbatively (if they 
are both consistent). We shall mention how this possibility can be checked in section 4.6.1 on $\mathrm{M}$ theory compactifications with flux. This subtlety involving the ordering of orientifold planes also appears for lower-dimensional orientifold configurations.

Therefore, we have a total of 4 distinct orientifold compactifications, and an additional 4 components in the $E_{8} \times E_{8}$ string moduli space. The standard $E_{8} \times E_{8}$ compactification together with the CHL string $/ \mathbb{Z}_{2}$-triple are contained in the orientifold moduli spaces.

\subsection{5 $D=6$}

In this dimension, all four kinds of orientifold planes are possible, and indeed each is realized in a particular $T^{4} / \mathbb{Z}_{2}$ orientifold. Let us first restrict our attention to $\mathrm{O5}^{-}$and $\mathrm{O5}^{-1}$ planes only. With this restriction, there are only two possibilities: one is $\left(-^{16}\right)$ and the other is $\left(-^{\prime 16}\right)$. The latter case is quite interesting. If we coalesce the $8 \mathrm{D}$-brane pairs on one of the $O^{-^{\prime}}$ planes, we get an $S O(17)$ maximal gauge group. The rank reduction is therefore 8. As discussed in appendix $\mathrm{C}$, the Chern-Simons invariant for any sub-three-torus is integer as required by anomaly cancellation. However, this compactification clearly has vector structure. The type I dual of this orientifold compactification therefore has a gauge bundle with vector structure which is not connected to the trivial bundle. We met this bundle in section 2.1.4, it is a non-trivial quadruple. Here we have found its orientifold realization.

In addition to these two cases, we have the dimensional reductions of the higher-dimensional cases which are $\left(-{ }^{12},+^{4}\right)$ and $\left(-{ }^{8},+^{8}\right)$. Recall that the first case includes the CHL string, the compactification with no vector structure and the $\mathbb{Z}_{2}$ triple in its moduli space. However, the duality chain of section 2.4.2 showed that the quadruple found above is in the same moduli space as the CHL string on $T^{4}$. Somewhat surprisingly, this implies that the $\left(-{ }^{12},+^{4}\right)$ and $\left(-^{\prime 16}\right)$ orientifolds are in the same moduli space. We can give 
further motivation for this inference in a simple way. Compactify both configurations to four dimensions on $T^{2}$. This gives $\left(-{ }^{48},+{ }^{16}\right)$ and $\left(-{ }^{16},-^{48}\right)$. S-duality maps $O^{-\prime} \leftrightarrow O^{+}$and $O^{-}$to itself. These configurations are therefore S-dual and their moduli spaces must necessarily agree.

Unlike the prior cases, we will not attempt a complete analysis of orientifold configurations in $D=6$. There are two other cases worth mentioning, however. The first is the case of $\left(-{ }^{10},+{ }^{6}\right)$ which made an appearance in [17]. This orientifold corresponds to a gauge bundle with $\widetilde{w}_{2}^{2}$ non-zero. The last case is $\left(+^{4},-^{\prime 12}\right)$. In this case, we need an additional 2 pairs of D-branes. The maximal gauge group is then $S p(2)$ and the rank reduction is 14 . Based on the structure of its moduli space, it is natural to conjecture that this orientifold is dual to the quadruple with no vector structure heterotic/type I compactification described in section 2.1.4. It would certainly be interesting to analyze this case further.

\subsection{6 $D=5$}

In this dimension, all four flavors of orientifold plane can again be realized by $T^{5} / \mathbb{Z}_{2}$ orientifolds. If we restrict our attention to $\mathrm{O4}^{-}$and $\mathrm{O}^{{ }^{-}}{ }^{\prime}$, there are only three possibilities up to diffeomorphisms: $\left(-{ }^{32}\right),\left(-^{\prime 32}\right)$ and $\left(-{ }^{16},-^{16}\right)$. We need a few additional words to actually describe these orientifolds. On a torus of sufficiently high dimension, the number of - and $-{ }^{\prime}$ planes does not in general completely specify the configuration up to diffeomorphisms. The actual pattern of the distribution must be specified. There is no room for such an ambiguity for $\left(-^{32}\right)$ and $\left(-^{\prime 32}\right)$ but there are several possibilities for $\left(-{ }^{16},-{ }^{16}\right)$. In this case, the only allowed configuration corresponds to the one where the $T^{5}$ can be factorized into $S^{1} \times T^{4}$ so that all the $O 4^{-}$planes sit at the 16 fixed points at the "origin" ( $\theta=0$ in natural coordinates) of the $S^{1}$. All the $O 4^{{ }^{\prime}}$ planes reside at the 16 fixed points in the "middle" of the $S^{1}(\theta=\pi)$.

We can find $\mathrm{M}$ theory duals for each of these cases. Recall that a single 
$O 4^{-}$plane at the origin of $\mathbb{R}^{5} / \mathbb{Z}_{2}$ is dual to $\mathrm{M}$ theory on $\mathbb{R}^{5} / \mathbb{Z}_{2} \times S^{1}$. Note that the $\mathbb{Z}_{2}$ action acts on the 3-form $C$ of $\mathrm{M}$ theory by inversion. On the other hand, $O 4^{{ }^{\prime}}$ is dual to $\mathrm{M}$ theory on $\left(\mathbb{R}^{5} \times S^{1}\right) / \mathbb{Z}_{2}$ where the $\mathbb{Z}_{2}$ acts on the last circle by a shift of a half period [46]. The dual of $\left(-{ }^{32}\right)$ is then M theory on $T^{5} / \mathbb{Z}_{2} \times S^{1}$ as we would naturally expect. The dual of $\left(-^{\prime 32}\right)$ is $\mathrm{M}$ theory on $\left(T^{5} \times S^{1}\right) / \mathbb{Z}_{2}$. The dual of $\left(--^{16},-{ }^{16}\right)$ is given by $\mathrm{M}$ theory on $\left(T^{5} \times S^{1}\right) / \mathbb{Z}_{2}$. The $\mathbb{Z}_{2}$ now acts on the coordinates $x_{1}, x_{2}, x_{3}, x_{4}, x_{5}, x_{11}$ of $T^{5} \times S^{1}$, where $x_{i} \equiv x_{i}+2 \pi$, by

$$
\left(x_{1}, x_{2}, x_{3}, x_{4}, x_{5}, x_{11}\right) \longrightarrow\left(-x_{1},-x_{2},-x_{3},-x_{4},-x_{5}, x_{11}+x_{1}\right)
$$

In the neighborhood of the "origin" $x_{1}=0$ of the first circle, the $\mathbb{Z}_{2}$ action is of the type $\mathbb{R}^{5} / \mathbb{Z}_{2} \times S^{1}$ and the O4-planes are all -. In the neighborhood of the "midpoint," $x_{1}=\pi$, the $\mathbb{Z}_{2}$ action is of the type $\left(\mathbb{R}^{5} \times S^{1}\right) / \mathbb{Z}_{2}$ and indeed the O4-planes are all $-^{\prime}$. We note that we would not be able to construct an $\mathrm{M}$ theory dual if other distributions of $16-$ and $16-^{\prime}$ planes were permitted.

As in the $D=6$ case, we shall only discuss select additional examples. From dimensional reduction, we obtain $\left(--^{24},+^{8}\right)$ and $\left(-{ }^{16},-^{16}\right)$. However, because of the duality explained in $D=6$, these should be part of the same moduli space. In addition, we have $\left(+{ }^{16},-{ }^{16}\right)$ and $\left(-{ }^{20},+{ }^{12}\right)$. Note that $\left(-^{\prime 32}\right)$ has no enhanced gauge symmetry so the rank reduction is 16 . Further, it does admit vector particles. This suggests that it is dual to type I with a non-trivial quintuple. This particular bundle made an appearance in section 2.1.5. By the chain of dualities in section 2.4.2, we see that this orientifold is further equivalent to a compactification of the $E_{8}$ string with a quintuple in both $E_{8}$ factors.

At first sight, it also seems plausible that $\left(-^{\prime 32}\right)$ could be identified with $\left(+{ }^{16},-{ }^{16}\right)$. By $\left(+{ }^{16},-{ }^{16}\right)$, we mean the configuration obtained by toroidally compactifying $(+,-)$ in nine dimensions. As support for this conjecture, note that on compactification to four dimensions, we find two configurations $\left(-{ }^{32},-^{32}\right)$ and $\left(-{ }^{32},+^{32}\right)$ which are S-dual. Of course, this alone does not 
demonstrate the equivalence. We can also study the $M$ theory description of $\left(+{ }^{16},{ }^{16}\right)$. Recall that the $D=9(+,-)$ orientifold is described, after T-duality, by IIB on $S^{1} / \delta \Omega$ where $\delta$ is a half-shift along the circle [17,52]. Let us compactify this configuration on a further $T^{4}$. We want to determine the corresponding $\mathrm{M}$ theory description. It is convenient to first compactify on one additional circle $S^{1}$. We can then T-dualize five times on the $T^{5}$ which leaves us in type IIA sending

$$
\delta \Omega \quad \rightarrow \quad \delta \Omega \mathbb{Z}_{2 \cdot}
$$

where the $\mathbb{Z}_{2}$ acts by inversion on the $T^{5}$. The operation $\Omega \mathbb{Z}_{2}$ lifts in $\mathrm{M}$ theory to inversion of the $T^{5}$ and the 3 -form $C$ [52]. This leaves us with $\mathrm{M}$ theory on $\left(T^{5} \times S^{1}\right) / \mathbb{Z}_{2} \times S^{1}$ where the $\mathbb{Z}_{2}$ acts as $\delta$ on the $S^{1}$ factor and by inversion on $T^{5}$. It also inverts the 3 -form $C$. This suggests that the $\mathrm{M}$ theory description of $\left(+{ }^{16},-{ }^{16}\right)$ is the same as the description of $\left(-^{\prime 32}\right)$ which is further evidence in favor of their equivalence.

\section{Compactifications of $M$ and F Theory}

\subsection{Some preliminary comments}

In prior sections, we have discussed aspects of perturbative string compactifications: either heterotic/type I or type II orientifolds. These descriptions are valid when the string coupling constant is small, regardless of the size of the compactification space. It is natural to ask what kind of description is valid when the string coupling constant is large. The answer to this question depends on how we treat the string scale $\alpha^{\prime}$ and the volume of the compactification space as $g_{s} \rightarrow \infty$. As an example, let us take the CHL string in 9 dimensions. If we wish to hold the 11-dimensional Planck scale fixed then $\alpha^{\prime} g_{s}^{2 / 3}$ must be held constant. In this limit, the strong coupling description will involve $\mathrm{M}$ theory compactified on a space which has been argued to be the Möbius strip [53]. This is analogous to the Horava-Witten description of the strongly-coupled $E_{8} \times E_{8}$ heterotic string. 
On the other hand, another strong coupling description of the $E_{8} \times E_{8}$ string on $T^{2}$ is given by $\mathrm{F}$ theory on $K 3$. When is this a valid description? Like $\mathrm{M}$ theory, $\mathrm{F}$ theory generically has no perturbative expansion and the condition for validity is that the base $B$ of the elliptic fibration (in this case $K 3$ ) be large in string units. It is convenient to analyze this relation at the point in the moduli space where the gauge group is broken to $\left(\operatorname{Spin}(8)^{4} \times\right.$ $\left.U(1)^{4}\right) / \mathbb{Z}_{2}^{3}$. Let the torus be square with volume $V$, and let $g_{H}$ denote the heterotic string coupling. T-duality along one cycle of the torus takes us to the $\operatorname{Spin}(32) / \mathbb{Z}_{2}$ heterotic string with ten-dimensional coupling $\left(g_{H}^{\prime}\right)^{2}=$ $g_{H}^{2} \alpha^{\prime} / V$ on a torus of volume $\alpha^{\prime}$. S-duality then takes us to the type I string with,

$$
g_{I}^{2}=\frac{V}{g_{H}^{2} \alpha^{\prime}}, \quad V_{I}=\frac{V}{g_{H}^{2}} .
$$

Two further T-dualities on the resulting torus take us to the type IIB on $T^{2} / \Omega(-1)^{F_{L}} \mathbb{Z}_{2}$, with couplings:

$$
g_{B}^{2}=\frac{\alpha^{\prime} g_{H}^{2}}{V}, \quad V_{B}=\frac{\left(\alpha^{\prime}\right)^{2} g_{H}^{2}}{V} .
$$

This is an orientifold limit of $\mathrm{F}$ theory on $K 3$ [54]. We see that $\mathrm{F}$ theory is a good description when $g_{H}$ becomes large with the volume $V$ fixed in string units. In this regime of the moduli space, we can use $\mathrm{F}$ theory to describe the physics.

A more general statement goes as follows. With sixteen supersymmetries, each component of the moduli space is highly constrained; for example, the moduli space metric does not receive quantum corrections. If the effective theory is formulated in $d$ space-time dimensions, then a given component of the moduli space can be described in terms of an even lattice $L$ of signature

$$
(s+10-d, 10-d) .
$$

(We use the convention in which there are more positive than negative eigenvalues in the lattice.) When $d>4$, this space takes the form

$$
\mathcal{M}_{L}:=O(L) \backslash \mathcal{D}_{L} \times \mathbb{R}^{+}
$$


where $\mathcal{D}_{L}=O(s+10-d, 10-d) /(O(s+10-d) \times O(10-d))$ is the symmetric space associated to the lattice, and $O(L)$ is the orthogonal group.

This must be modified somewhat in low dimension: when $d=4$, the universal cover of the given component of the moduli space is $\mathcal{D}_{L} \times \mathfrak{h}$ where $\mathfrak{h}$ is the upper half plane, and when $d=3$, the universal cover of the given component is $\mathcal{D}_{\widetilde{L}}$, where $\widetilde{L}$ is the direct sum of $L$ and a lattice of signature $(1,1)$.

A given moduli space has boundaries that correspond to the various ways in which a theory can degenerate. A different physical description is typically valid as we approach a boundary of the moduli space. Our goal in this section is to study a class of $\mathrm{M}$ and $\mathrm{F}$ theory compactifications which naturally include dual descriptions for the perturbative compactifications described in earlier sections. These include purely geometric models and also models with background fluxes, as in the case studied by Schwarz and Sen [14].

We now require a more general discussion of the boundaries of moduli spaces than appeared in our initial discussion of section 2.4.3. The possible boundary components of $\mathcal{M}_{L}$ are determined as follows (setting $d \geq 5$ for simplicity). One type of boundary is given by approaching one end or the other of the $\mathbb{R}^{+}$factor. These include non-stringy limits: for example, in a conventional heterotic or type II compactification one of these limits is the zero-coupling limit which yields a conformal field theory rather than a string theory. This is the kind of description that we studied in prior sections. As discussed above, the strong coupling limit will typically have an $\mathrm{M}$ or $\mathrm{F}$ theory description. Let us here instead focus on the other class of limits, given by boundary components of the $O(L) \backslash \mathcal{D}_{L}$ factor. These boundary components typically correspond to a limiting stringy theory whose effective dimension is greater than $d$.

A boundary component of $O(L) \backslash \mathcal{D}_{L}$ is determined by an isotropic sublattice $M \subset L$, that is, every $x \in M$ satisfies $q(x)=0$. The lattice associated to 
the boundary component is then given by $L_{M}:=M^{\perp} / M$, and the boundary component takes the form

$$
O\left(L_{M}\right) \backslash \mathcal{D}_{L_{M}}
$$

where we suppress the $\mathbb{R}^{+}$factor. To determine all boundary components, all isotropic sublattices $M$ must be found, modulo the action of the orthogonal group $O(L)$. If the sublattice $M$ has rank $m$, the limiting theory will have effective dimension $d+m$.

In general, $L_{M}$ only determines part of a component of the moduli space of the limiting theory in effective dimension $d+m$. That is (suppressing the $\mathbb{R}^{+}$factors), the space $O(L) \backslash \mathcal{D}_{L}$ is glued to a space $O\left(L^{\prime}\right) \backslash \mathcal{D}_{L^{\prime}}$ along the boundary component $O\left(L_{M}\right) \backslash \mathcal{D}_{L_{M}}$, where $L^{\prime}$ is a lattice of signature $\left(s^{\prime}+10-(d+m), 10-(d+m)\right)$ which represents the limiting effective theory. The gluing is specified by an inclusion $L_{M} \subset L^{\prime}$ with $L^{\prime} / L_{M}$ a positive definite lattice of rank $s^{\prime}-s \geq 0$. The lattice $L^{\prime}$ must be determined by analyzing the physics of the limiting process; it agrees with $L_{M}$ for some boundary components but is larger than $L_{M}$ for others.

For example, the CHL string in nine dimensions has lattice [38] $L \cong$ $\Gamma_{1,1} \oplus E_{8}$. There is a unique boundary component, corresponding to $L_{x} \cong$ $E_{8}$. However, as we discussed in section 2.4.3, in the decompactification limit we actually obtain the heterotic string in ten dimensions with lattice $L^{\prime} \cong E_{8} \oplus E_{8}$.

Decompactification limits of components corresponding to non-trivial discrete choices of Wilson lines exhibit a similar phenomenon: viewed from the perspective of the higher-dimensional theory, the non-trivial types of Wilson lines can only be turned on for special values of the moduli. Thus, it is along a subspace $O\left(L_{M}\right) \backslash \mathcal{D}_{L_{M}}$ of $O\left(L^{\prime}\right) \backslash \mathcal{D}_{L^{\prime}}$ that the moduli space of the lower dimensional theory "attaches," and one recovers additional degrees of freedom in the decompactification limit.

A similar phenomenon occurs in $\mathrm{F}$ theory [13], where compactification 
along an additional circle gives theories which are dual to $\mathrm{M}$ theory on elliptically fibered manifolds. The discrete Wilson line degrees of freedom correspond to the possibility of compactifying $M$ theory on an elliptically fibered manifold without a section, which typically is only possible for special values of the moduli.

\subsection{Six-dimensional $M$ theory compactifications without fluxes}

Our starting point is $\mathrm{M}$ theory compactified to 6 dimensions, but we will also include remarks about lower-dimensional compactifications. ${ }^{10}$ Let us begin by excluding any background flux so that this is a purely geometric compactification. We shall also restrict to supersymmetric compactifications which take the form of a compact Riemannian manifold times Minkowski space. The metric on the compact part must then admit a covariantly constant spinor, which leads to restrictions on the holonomy. In fact, the list of possibilities can be determined by examining the holonomy classification of Riemannian metrics, which (when formulated carefully [55] ${ }^{11}$ ) implies that every compact Riemannian manifold $Y$ admitting a covariantly constant spinor takes the form

$$
Y=\left(T^{k} \times\left(X_{1} \times \cdots \times X_{m}\right) / \Gamma\right) / G,
$$

where $T^{k}$ is a torus of dimension $k \geq 0$, each $X_{i}$ is a compact simplyconnected Riemannian manifold whose holonomy is either $S U\left(n_{i}\right), S p\left(n_{i}\right)$, $G_{2}$, or $\operatorname{Spin}(7)$, and $\Gamma$ and $G$ are finite groups which act without fixed points. The effective dimension of the physical theory is $d=11-\operatorname{dim} Y$.

In order to guarantee at least 16 supercharges in the effective theory, there must be at least half as many holonomy-invariant spinors on this manifold as there are on flat space. Because each $X_{i}$ in eq. (70) reduces the set of holonomy-invariant spinors by at least a factor of two, and the factor is greater than two except in the case of holonomy $S U(2)=S p(1)$, there are

\footnotetext{
${ }^{10}$ The corresponding type IIA string compactifications were studied in detail in $[41,42]$.

${ }^{11}$ We thank B. McInnes for helpful correspondence on this point.
} 
two cases: either (1) there is a single $X_{i}$ with holonomy $S U(2)=S p(1)$ (i.e., a K3 surface), the group $\Gamma$ is trivial, and the group $G$ preserves all of the spinors on $T^{k} \times X$, or (2) there is no $X_{i}$ at all (and hence no $\Gamma$ ) and the group $G$ preserves one-half of the spinors on $T^{k}$. In the second case, possibly after replacing the torus by a finite cover or a finite quotient, we can assume that [56] $T^{k}=T^{4} \times T^{k-4}$ with the group action preserving the 1-forms on $T^{k-4}$ and the holomorphic 2-form on $T^{4}$, but leaving no invariant 1-forms on $T^{4}$. To get the correct holonomy, the image of $G$ in $S O(k)$ must lie in an $S U(2)$ subgroup corresponding to a complex structure on the $T^{4}$ factor. In both cases, then, we can write $Y=\left(T^{\ell} \times Z\right) / G$ where $Z$ is either a $\mathrm{K} 3$ surface or a complex 2-torus (that is, a real 4-torus on which a complex structure has been specified).

The lattice $L$ can be directly determined from the cohomology of $Y$. When $d>4$, the possible gauge charges for the theory are described by

$$
H^{1}(Y, \mathbb{Z}) \oplus H^{2}(Y, \mathbb{Z}) \oplus H^{5}(Y, \mathbb{Z}),
$$

(with the first factor coming from Kaluza-Klein modes, and the latter two coming from the $\mathrm{M}$ theory three-form and its dual six-form). This cohomology group comes equipped with a natural quadratic form, to be described below. Bearing in mind the sign conventions, we can identify the free part of eq. (71) with the lattice $L(-1)$ if $d>5$. (There is also the possibility of torsion in eq. (71), which we will not explore in any detail.) When $d=5$, the free part of the gauge lattice in eq. (71) takes the form $L(-1) \oplus\langle x\rangle$ with $q(x)=0$; the element $x$ is unique up to \pm 1 , so $L(-1)$ can be recovered by modding out the free part of the gauge lattice by the span of $x$. When $d=4$, the free part of the gauge lattice is simply

$$
H^{1}(Y, \mathbb{Z}) \oplus H^{2}(Y, \mathbb{Z})
$$

due to the self-duality of gauge fields in this dimension, and this coincides with $L(-1)$.

The description of the quadratic form on $L$ depends on the dimension $d$ of the effective theory. If $d=6$, both $H^{1}(Y)$ and $H^{5}(Y)$ are 1-dimensional 


\begin{tabular}{|l|l|c|}
\hline$k$ & $G$ & $\begin{array}{c}\text { Maximum dimension } \\
\text { of effective theory }\end{array}$ \\
\hline 0 & $\{e\}$ & 7 \\
1 & $\mathbb{Z}_{m}, m=2,3,4,5,6,7,8$ & 6 \\
2 & $\mathbb{Z}_{2} \times \mathbb{Z}_{m}, m=2,4,6$ or & \\
& $\mathbb{Z}_{m} \times \mathbb{Z}_{m}, m=3,4$ & 5 \\
3 & $\left(\mathbb{Z}_{2}\right)^{3}$ & 4 \\
4 & $\left(\mathbb{Z}_{2}\right)^{4}$ & 3 \\
\hline
\end{tabular}

Table 7: Automorphisms of K3 surfaces and the resulting M theory vacua.

and this part of the lattice is isomorphic to $\Gamma_{1,1}$. The quadratic form on $H^{2}(Y, \mathbb{Z}) /$ torsion is inherited from the intersection form on the resolution $\widetilde{Z / G}$ of $Z / G$ via the isomorphism

$$
\begin{aligned}
H^{2}(Y, \mathbb{Z}) / \text { torsion } \cong & H^{2}(Z / G, \mathbb{Z}) / \text { torsion } \\
\cong & \text { the orthogonal complement of the } \\
& \quad \text { exceptional divisors in } H^{2}(\widetilde{Z / G}, \mathbb{Z}) .
\end{aligned}
$$

Thus, although the action of $G$ on $S^{1} \times Z$ has no fixed points and a smooth quotient, keeping track of the singular points on $Z / G$ provides a convenient bookkeeping device for analyzing the lattice associated to $\left(S^{1} \times Z\right) / G$. There is one subtlety associated to this, however. If $E \subset H^{2}(\widetilde{Z / G}, \mathbb{Z})$ denotes the lattice spanned by the exceptional divisors, then $\left(E^{\perp}\right)^{\perp}$ will be larger than $E$ : there are $\mathbb{Q}$-linear combinations of exceptional divisors which belong to $H^{2}(\widetilde{Z / G}, \mathbb{Z})$. In fact, the finite group $\left(E^{\perp}\right)^{\perp} / E$ provides another important invariant in this situation.

Consider first the case in which $Y=\left(T^{\ell} \times Z\right) / G$ where $Z$ is a K3 surface. The action of $G$ preserves the two factors $T^{\ell}$ and $Z$. In order to preserve the invariant spinors on $T^{\ell}$ it must act by translations on that factor, and in order to preserve the invariant spinors on $Z$ it must preserve the holomorphic 2-form on $Z$. Abelian group actions which preserve the holomorphic 2-form 


\begin{tabular}{|c|c|c|c|}
\hline$G$ & Lattice $L$ & Singularities on $\mathrm{K} 3 / G$ & $\left(E^{\perp}\right)^{\perp} / E$ \\
\hline $\mathbb{Z}_{1}$ & $\Gamma_{4,4} \oplus E_{8} \oplus E_{8}$ & none & $\{e\}$ \\
$\mathbb{Z}_{2}$ & $\Gamma_{1,1}(2) \oplus \Gamma_{3,3} \oplus D_{4} \oplus D_{4}$ & $8 A_{1}$ & $\mathbb{Z}_{2}$ \\
$\mathbb{Z}_{3}$ & $\Gamma_{1,1}(3) \oplus \Gamma_{3,3} \oplus A_{2} \oplus A_{2}$ & $6 A_{2}$ & $\mathbb{Z}_{3}$ \\
$\mathbb{Z}_{4}$ & $\Gamma_{1,1}(4) \oplus \Gamma_{3,3} \oplus A_{1} \oplus A_{1}$ & $4 A_{3}+2 A_{1}$ & $\mathbb{Z}_{4}$ \\
$\mathbb{Z}_{5}$ & $\Gamma_{1,1}(5) \oplus \Gamma_{3,3}$ & $4 A_{4}$ & $\mathbb{Z}_{5}$ \\
$\mathbb{Z}_{6}$ & $\Gamma_{1,1}(6) \oplus \Gamma_{3,3}$ & $2 A_{5}+2 A_{2}+2 A_{1}$ & $\mathbb{Z}_{6}$ \\
$\mathbb{Z}_{7}$ & $\Gamma_{2,2} \oplus\left(\begin{array}{cc}28 & 7 \\
7 & 2\end{array}\right)$ & $3 A_{6}$ & \\
$\mathbb{Z}_{8}$ & $\Gamma_{2,2} \oplus\left(\begin{array}{cc}4 & 0 \\
0 & 2\end{array}\right)$ & $2 A_{7}+A_{3}+A_{1}$ & $\mathbb{Z}_{7}$ \\
\hline
\end{tabular}

Table 8: Choices for $G$ together with their associated lattices for $Y=\left(S^{1} \times\right.$ $K 3) / G$.

on $Z$ were classified by Nikulin [37]; there are 15 cases, including the trivial group. The resulting vacua are displayed in table 7 , where $k$ denotes the number of generators in the group, and hence the minimal dimension of a torus factor in $Y$.

The lattices $H^{2}(Z / G)$ in these cases are also known [37]. In table 8 we exhibit the lattices $L$ associated to six-dimensional effective theories built from M theory on $Y=\left(S^{1} \times K 3\right) / G$ when $G$ is trivial or cyclic; ${ }^{12}$ we also describe the singularities which are found on $Z / G$ itself, using the $A D E$ notation for rational double points, and the finite group $\left(E^{\perp}\right)^{\perp} / E$ where $E$ is the sublattice of $H^{2}(\widetilde{Z / G}, \mathbb{Z})$ spanned by the exceptional divisors. The corresponding facts about non-cyclic groups $G$ (where the effective theory has lower dimension) are given in table 9. (The "discriminant group" which appears in that table is discussed in appendix A.)

\footnotetext{
${ }^{12}$ The descriptions we give of the lattices can be inferred from the descriptions in [37] using techniques from [57].
} 


\begin{tabular}{|c|c|c|c|}
\hline $\begin{array}{c}\text { Compactification } \\
\text { dimension }\end{array}$ & $G$ & $\begin{array}{c}\text { Rank of } \\
H^{2}(K 3 / G)\end{array}$ & $\begin{array}{c}\text { Discriminant } \\
\text { group }\end{array}$ \\
\hline 5 & $\mathbb{Z}_{2} \times \mathbb{Z}_{2}$ & 10 & $\left(\mathbb{Z}_{2}\right)^{8}$ \\
5 & $\mathbb{Z}_{2} \times \mathbb{Z}_{4}$ & 6 & $\left(\mathbb{Z}_{2}\right)^{2} \times\left(\mathbb{Z}_{4}\right)^{2}$ \\
5 & $\mathbb{Z}_{2} \times \mathbb{Z}_{6}$ & 4 & $\left(\mathbb{Z}_{2}\right) \times\left(\mathbb{Z}_{6}\right)$ \\
5 & $\mathbb{Z}_{3} \times \mathbb{Z}_{3}$ & 6 & $\left(\mathbb{Z}_{3}\right)^{4}$ \\
5 & $\mathbb{Z}_{4} \times \mathbb{Z}_{4}$ & 4 & $\left(\mathbb{Z}_{4}\right)^{2}$ \\
4 & $\mathbb{Z}_{2} \times \mathbb{Z}_{2} \times \mathbb{Z}_{2}$ & 8 & $\left(\mathbb{Z}_{2}\right)^{8}$ \\
3 & $\mathbb{Z}_{2} \times \mathbb{Z}_{2} \times \mathbb{Z}_{2} \times \mathbb{Z}_{2}$ & 7 & $\left(\mathbb{Z}_{2}\right)^{7}$ \\
\hline
\end{tabular}

Table 9: Choices for $G$ for lower-dimensional compactifications.

Turning to the case where $Y=\left(T^{\ell} \times Z\right) / G$ with $Z$ a complex 2-torus, we need an abelian group $G$ acting on $Z$ in such a way that the holomorphic 2 -form is preserved by the $G$-action. Moreover, the group action must not leave any holomorphic 1-form invariant, so $G$ cannot act entirely by translations. Such group actions were classified in modern language by Fujiki [56] (although the classification was essentially done more than ninety years ago by Enriques and Severi [58]). The computations of the corresponding lattices in the case of abelian group actions were made in [59-61] and were applied in the physics literature in [62]. The only possibilities are cyclic groups $G=\mathbb{Z}_{m}$ with $m=2,3,4$, or 6 , and all of these lead to theories of effective dimension six. The associated lattices are described in table $10,{ }^{13}$ where we also give the singularities on $Z / G$ and the finite group $\left(E^{\perp}\right)^{\perp} / E$.

\subsection{F theory compactifications without flux}

It is common to describe an $\mathrm{F}$ theory vacuum in terms of a Ricci-flat manifold $Y$ together with an elliptic fibration $\pi: Y \rightarrow B$. However, to specify an $\mathrm{F}$

\footnotetext{
${ }^{13}$ Again, matching the descriptions in table 10 with those in [59-61] requires techniques from [57].
} 


\begin{tabular}{|c|c|c|c|}
\hline$G$ & Lattice $L$ & Singularities on $T^{4} / G$ & $\left(E^{\perp}\right)^{\perp} / E$ \\
\hline $\mathbb{Z}_{2}$ & $\Gamma_{3,3}(2) \oplus \Gamma_{1,1}$ & $16 A_{1}$ & $\left(\mathbb{Z}_{2}\right)^{5}$ \\
$\mathbb{Z}_{3}{ }^{\prime}$ & $\Gamma_{1,1}(3) \oplus \Gamma_{1,1} \oplus\left(\begin{array}{ll}2 & 1 \\
1 & 2\end{array}\right)$ & $9 A_{2}$ & $\left(\mathbb{Z}_{3}\right)^{3}$ \\
$\mathbb{Z}_{4}$ & $\Gamma_{1,1}(4) \oplus \Gamma_{1,1} \oplus\left(\begin{array}{ll}2 & 0 \\
0 & 2\end{array}\right)$ & $4 A_{3}+6 A_{1}$ & $\mathbb{Z}_{4} \times\left(\mathbb{Z}_{2}\right)^{2}$ \\
$\mathbb{Z}_{6}$ & $\Gamma_{1,1}(6) \oplus \Gamma_{1,1} \oplus\left(\begin{array}{ll}6 & 0 \\
0 & 2\end{array}\right)$ & $A_{5}+4 A_{2}+5 A_{1}$ & $\mathbb{Z}_{6}$ \\
\hline
\end{tabular}

Table 10: Choices for $G$ together with their associated lattices for $Y=$ $\left(S^{1} \times T^{4}\right) / G$.

theory vacuum, we actually only need

1. the manifold $B$ with a subset $\Delta$ of real codimension 2 (where $\Delta$ specifies the location of the singular fibers in the fibration $\pi$ ),

2. a monodromy representation $\pi_{1}(B-\Delta, p) \rightarrow S L(2, \mathbb{Z})$ and a " $j$-function" $j: B \rightarrow \mathbb{C P}^{1}$ compatible with the monodromy (which are specified by the complex structure on the fibers of $\pi$ ), and

3. a metric on $B-\Delta$ whose asymptotics near $\Delta$ are described by the Greene-Shapere-Vafa-Yau ansatz [63] (which can be seen as a limit of metrics on $Y$ as the area of the elliptic fiber approaches zero [64]).

The $\mathrm{F}$ theory vacuum is then described as type IIB string theory compactified on $B$ with the given metric and with branes along $\Delta$, using the S-duality of type IIB theory to compensate for the $S L(2, \mathbb{Z})$ monodromy.

If we begin from $\mathrm{M}$ theory compactified on $Y$, and take the limit as the area of the fibers of $\pi$ approaches zero, then one dimension of the effective theory decompactifies $[42,65]$ and we obtain the $\mathrm{F}$ theory vacuum in the limit. This is sometimes referred to as " $\mathrm{F}$ theory compactified on $Y$ " although the 
full data of $Y$ is not needed. Conversely, if the elliptic fibration on $Y$ has a section, then the standard $\mathrm{M}$ theory/F theory duality [66] asserts that $\mathrm{F}$ theory on $Y \times S^{1}$ (with a trivial Wilson line) is dual to M theory on $Y$.

When the elliptic fibration on $Y$ does not have a section, there is always an associated manifold $\mathcal{J}(Y)$, the Jacobian of the fibration, which has an elliptic fibration with a section that gives rise to the same monodromy and $j$-function data as the elliptic fibration on the original manifold. Thus, F theory cannot distinguish between the compactification on $Y$ and the compactification on $\mathcal{J}(Y)$.

The $\mathrm{M}$ theory/F theory duality can be extended to cover this case [13], where it becomes the assertion that when Wilson line data is included, $\mathrm{F}$ theory on $\mathcal{J}(Y) \times S^{1}$ is dual to the union of the $\mathrm{M}$ theory moduli spaces on $Y_{k}$ for all manifolds $Y_{k}$ with the same Jacobian fibration $\mathcal{J}\left(Y_{k}\right)=\mathcal{J}(Y){ }^{14}$ Thus, discrete choices for Wilson lines in $\mathrm{F}$ theory correspond in $\mathrm{M}$ theory to different elliptic fibrations with the same Jacobian fibration. Typically, such discrete choices are only present for special values of moduli.

An elliptic fibration on a Ricci-flat manifold $Y$ always determines a class $x \in H^{2}(Y, \mathbb{Z})$ with $q(x)=0$. Thus, in the case of 16 supercharges, the boundary lattice associated to taking the $\mathrm{F}$ theory limit is $L_{x}=x^{\perp} /\langle x\rangle$. If the elliptic fibration admits a section, then this lattice can be used to describe the entire component of the $\mathrm{F}$ theory moduli space. On the other hand, if the elliptic fibration does not admit a section, then the lattice $L^{\prime}$ for the component of $\mathrm{F}$ theory moduli space associated to $\mathcal{J}(Y)$ is typically larger than $L_{x}$.

To find these components in detail, we need to examine possible elliptic fibrations on the Ricci-flat manifolds $Y=\left(T^{\ell} \times Z\right) / G$ (with $Z$ either a K3 surface or a $\left.T^{4}\right)$. An elliptic fibration $\pi:\left(T^{\ell} \times Z\right) / G \rightarrow B$ will lift to an elliptic fibration $\tilde{\pi}: T^{\ell} \times Z \rightarrow \widetilde{B}$ which is $G$-invariant. If $\pi$ has a section then its inverse image will be a $G$-invariant section of $\widetilde{\pi}$. The group $G$ acts on the

\footnotetext{
${ }^{14}$ For other comments on $\mathrm{F}$ theory compactifications without section, see [67].
} 
base $\widetilde{B}$ and $B=\widetilde{B} / G$. We let $G_{0}$ be the subgroup of $G$ which acts trivially on the base $\widetilde{B}$. It is easy to see that if $G_{0}$ is non-trivial, there cannot be a section for the fibration $\pi$ : the group $G_{0}$ acts by translations on the fibers, so cannot preserve a section of $\widetilde{\pi}$, but $\widetilde{\pi}$ must have a $G$-invariant section when $\pi$ has a section. Thus, when $\pi$ has a section the action of $G$ on $\widetilde{B}$ must be faithful.

Let us first analyze the cases in which $\pi$ does not have a section. As indicated above, the lack of a section can be attributed to a non-trivial group $G_{0}$ which acts trivially on the base $\widetilde{B}$. Let us begin with the case $Z=K 3$ and return to the case $Z=T^{4}$ in a little while. In fact, it is easy to see that the Jacobian of the elliptic fibration on $\left(T^{\ell} \times Z\right) / G_{0}$ is just $T^{\ell} \times\left(Z / G_{0}\right)$. The manifold $Z / G_{0}$ is a singular $\mathrm{K} 3$ surface, and this is in fact part of a larger family of manifolds of the form $T^{\ell} \times K 3$. The possible $G_{0}$ 's which can occur here can be classified: we need to know which finite abelian groups $G_{0}$ could act as a group of translations on the fibers of an elliptic fibration on a K3 surface. This classification was carried out by Cox [68], who found almost exactly the same list as Nikulin's classification of abelian automorphism groups [37], except that $(\mathbb{Z} / 2 \mathbb{Z})^{3}$ and $(\mathbb{Z} / 2 \mathbb{Z})^{4}$ cannot occur as translations on the fibers. Thus, in all of these cases, there is a limit of the $\mathrm{M}$ theory moduli space in which the limiting $\mathrm{F}$ theory vacua gain additional degrees of freedom which allow them to be part of the "standard component" of the F theory moduli space on $S^{1} \times K 3$. Since the lattice of the component of $\mathrm{M}$ theory moduli space takes the form $L(-1) \cong \Gamma_{1,1} \oplus H^{2}\left(Z / G_{0}, \mathbb{Z}\right)$, we must have $L_{x}(-1)=H^{2}\left(Z / G_{0}, \mathbb{Z}\right)$ in order to allow the lattice $L_{x}$ to be embedded into the "standard lattice" $\Gamma_{3,3} \oplus E_{8} \oplus E_{8}$. This is exactly the type of lattice limit which is found for the standard component.

There can be "mixed" cases as well, in which both $G_{0}$ and $G_{1}:=G / G_{0}$ are non-trivial. In such a case, the Jacobian of the elliptic fibration on $\left(T^{\ell} \times\right.$ $Z) / G$ will be an elliptic fibration on $\left(T^{\ell} \times\left(Z / G_{0}\right)\right) / G_{1}$. The limiting theory "attaches" to the moduli space $\left(T^{\ell} \times Z^{\prime}\right) / G_{1}$, and the lattice decomposition $L \cong M_{1} \oplus L_{x}$ should use the rank two lattice $M_{1}$ associated with $G_{1}$. (We 

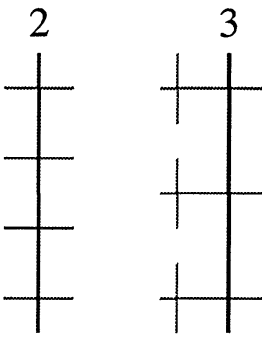

$3 A_{2}$

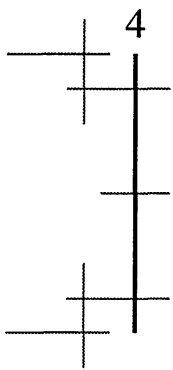

$2 A_{3}+A_{1}$

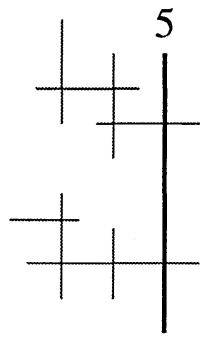

$2 A_{4}$

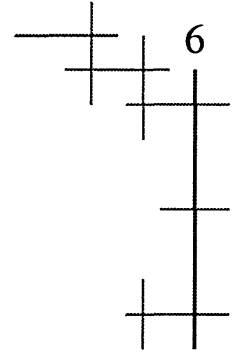

$A_{5}+A_{2}+A_{1}$

Figure 3: The singular fibers on $Z / G$.

will determine those lattices below.)

Turning now to the cases in which $\pi$ has a section, there will be a component of the $\mathrm{F}$ theory moduli space for each case in which $\pi$ has a section. When $Z$ is a $\mathrm{K} 3$ surface, both $B$ and $\widetilde{B}$ are isomorphic to $\mathbb{C P}^{1}$ so $G$ must have a faithful action on $\mathbb{C P}^{1}$, that is, there must be an injective homomorphism $G \rightarrow S O(3)$. Since $G$ is abelian, the only possibilities are that $G$ is trivial, or $G$ is cyclic, or $G \cong\left(\mathbb{Z}_{2}\right)^{2}$.

When $G$ is trivial, we can take $\ell=0$ and we get the "standard" $\mathrm{F}$ theory component in 8 dimensions. This leads to standard components in lower dimensions as well, which can be treated as $\mathrm{F}$ theory on $T^{\ell} \times K 3$.

When $G \cong \mathbb{Z}_{m}$ is cyclic, there will be two fixed points for the action of $G$ on $\widetilde{B}=\mathbb{C P}^{1}$. All of the fixed points for the action of $G$ on $Z$ must lie in one of the two elliptic curves fixed by the $G$-action, and the quotient $Z / G$ will have an elliptic fibration which degenerates to have a irreducible fiber of multiplicty $m$ at each of the two fixed points. Note that $Z / G$ has singularities along these two fibers as well, and that the resolved surface $\widetilde{Z / G}$ will have a more conventional elliptic fibration, with section (since there is a section up on $Z$ by assumption). Since the only multiplicities which can occur within fibers in such a fibration are $m \leq 6$, we learn that the only possible cyclic group actions in this case are $\mathbb{Z}_{m}$ with $2 \leq m \leq 6$. 

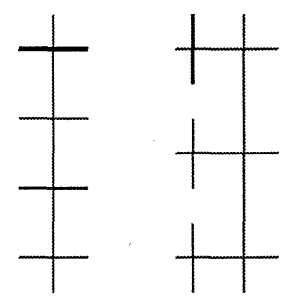

$I_{0}^{*}$

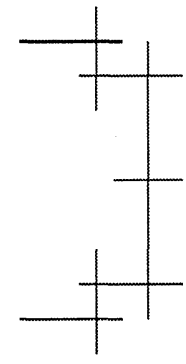

$I I I^{*}$

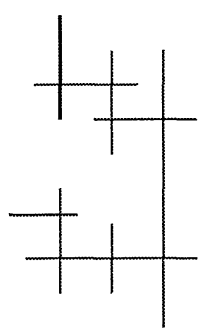

$I I^{*}$

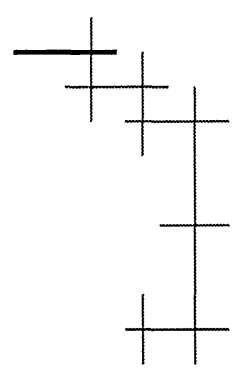

$I I^{*}$

Figure 4: The singular fibers for $\mathrm{F}$ theory on $\widetilde{Z / G}$.

In fact, it is possible to see the geometry of these group actions quite explicitly. We have already enumerated the possible singular points on $Z / G$ in table 8. These singular points are grouped together into elliptic fibers as indicated in figure 3. In each case, the elliptic curves on $Z / G$ degenerate to an irreducible curve passing through 2,3 , or 4 singular points, and the irreducible curve has multiplicity $m$ in the elliptic fibration on $Z / G$. All of the values $2 \leq m \leq 6$ do occur, as shown in figure $3 .^{15}$ On each fiber in the figure, the thick (blue) line represents the irreducible curve (which is labeled by its multiplicity $m$ in the fiber), and the thin (red) lines represent the curves in the resolutions of the various singularities. We have labeled each fiber with the types of singular points that occur on it.

We remind the reader that the singularities of $Z / G$ do not actually occur in our $\mathrm{M}$ theory and $\mathrm{F}$ theory vacua, which are compactified on $\left(S^{1} \times Z\right) / G$; the surface $Z / G$ is just a convenient device for determining the lattice $L$ of the $\mathrm{M}$ theory compactification. To determine the lattice of the $\mathrm{F}$ theory compactification, we should use a different birational model of $Z / G$ : either the nonsingular surface $\widetilde{Z / G}$, or the Weierstrass model obtained from $\widetilde{Z / G}$ by blowing down all components of fibers other than the ones meeting a

\footnotetext{
${ }^{15} \mathrm{~A}$ related geometric structure appears in [69]. It would be interesting to understand how this is related to the frozen singularities that we will later discuss.
} 


\begin{tabular}{|c|c|c|}
\hline$G$ & Lattice $L_{x}$ & Singular fibers on $\widetilde{K 3 / G}$ \\
\hline $\mathbb{Z}_{1}$ & $\Gamma_{3,3} \oplus E_{8} \oplus E_{8}$ & none \\
$\mathbb{Z}_{2}$ & $\Gamma_{3,3} \oplus D_{4} \oplus D_{4}$ & $I_{0}^{*}+I_{0}^{*}$ \\
$\mathbb{Z}_{3}$ & $\Gamma_{3,3} \oplus A_{2} \oplus A_{2}$ & $I V^{*}+I V^{*}$ \\
$\mathbb{Z}_{4}$ & $\Gamma_{3,3} \oplus A_{1} \oplus A_{1}$ & $I I I^{*}+I I I^{*}$ \\
$\mathbb{Z}_{5}$ & $\Gamma_{3,3}$ & $I I^{*}+I I^{*}$ \\
$\mathbb{Z}_{6}$ & $\Gamma_{3,3}$ & $I I^{*}+I I^{*}$ \\
\hline
\end{tabular}

Table 11: $\mathrm{F}$ theory lattices and singular fibers for $Y=\left(S^{1} \times K 3\right) / G$.

section. ${ }^{16}$ Each singular fiber can then be labeled by its Kodaira type; the labels for the fibers for different values of $m$ are shown in figure 4 . The fibers in that figure are in one-to-one correspondence with the fibers in figure 3 , and for each fiber in figure 4 , the thick (blue) line represents the fiber component which meets the section, and the thin (red) lines represent the components which are blown down to give the Weierstrass model.

The configurations of singular points on $Z / G$ from table 8 are thus collected into Kodaira fibers, giving the results in the right hand column of table $11 .{ }^{17}$ From the Kodaira fibers, a lattice can easily be computed, as shown in the middle column of table 11, and we claim that this is the lattice which describes the moduli space for the corresponding $\mathrm{F}$ theory component. In fact, this same lattice can be arrived at in two ways: either directly in terms of the Kodaira fibers for the elliptic fibration on $\widetilde{Z / G}$, or by using the $x^{\perp} /\langle x\rangle$ construction, using the fact that $L=\Gamma_{1,1}(m) \oplus L_{x}$ in the case that $Y=\left(S^{1} \times Z\right) / G$. (This latter result becomes obvious when comparing tables 8 and 11.) This gives six different $F$ theory vacua. These vacua

\footnotetext{
${ }^{16}$ Again, the singularities on the Weierstrass model do not directly show up in our $\mathrm{F}$ theory vacuum, but they will reappear as "frozen singularities" in an M theory limit with 3-form flux described in section 4.6.1.

${ }^{17}$ Note that the free part of the lattices for the $\mathbb{Z}_{5}$ and $\mathbb{Z}_{6}$ cases appearing table 11 are identical. It would be interesting to check whether the full cohomology lattices differ by torsion classes.
} 


\begin{tabular}{|c|c|c|}
\hline$G$ & Lattice for $\widetilde{K 3 / G}$ & Singular fibers on $\widetilde{K 3 / G}$ \\
\hline$\left(\mathbb{Z}_{2}\right)^{2}$ & $\Gamma_{2,2} \oplus \Gamma_{1,1}(2) \oplus D_{4}$ & $I_{0}^{*}+I_{0}^{*}+I_{0}^{*}$ \\
\hline
\end{tabular}

Table 12: The additional $\mathrm{F}$ theory vacuum in dimension 6 .

\begin{tabular}{|c|c|c|}
\hline$G$ & Lattice $L_{x}$ & Singular fibers on $\widehat{T^{4} / G}$ \\
\hline $\mathbb{Z}_{2}$ & $\Gamma_{2,2}(2) \oplus \Gamma_{1,1}$ & $I_{0}^{*}+I_{0}^{*}+I_{0}^{*}+I_{0}^{*}$ \\
$\mathbb{Z}_{3}$ & $\left(\begin{array}{ll}2 & 1 \\
1 & 2\end{array}\right) \oplus \Gamma_{1,1}$ & $I V^{*}+I V^{*}+I V^{*}$ \\
$\mathbb{Z}_{4}$ & $\left(\begin{array}{ll}2 & 0 \\
0 & 2\end{array}\right) \oplus \Gamma_{1,1}$ & $I I I^{*}+I I I^{*}+I_{0}^{*}$ \\
$\mathbb{Z}_{6}$ & $\left(\begin{array}{ll}6 & 0 \\
0 & 2\end{array}\right) \oplus \Gamma_{1,1}$ & $I I^{*}+I V^{*}+I_{0}^{*}$ \\
\hline
\end{tabular}

Table 13: F theory lattices and singular fibers for $Y=\left(S^{1} \times T^{4}\right) / G$.

are dual to the six heterotic asymmetric orbifolds in 7 dimensions that we constructed in section 2 .

Note that in the two remaining cyclic cases for $M$ theory vacua, namely $\mathbb{Z}_{7}$ and $\mathbb{Z}_{8}$, it is not possible to split off a factor of $\Gamma_{1,1}(m)$ from the lattice $L$ as given in table 8 . This gives further confirmation that there are no $\mathrm{F}$ theory vacua associated with these cases.

The one remaining $\mathrm{F}$ theory vacuum with $Z=K 3$ is associated to the group $G=\mathbb{Z}_{2} \times \mathbb{Z}_{2}$; see table 12 . The lattice we list is for $K 3 / G$. To this lattice, we must add a lattice of signature $(2,2)$ for the 5 -dimensional $\mathrm{M}$ theory compactification and a lattice of signature $(1,1)$ for the $\mathrm{F}$ theory compactification. The fixed points for the action of $G$ on $\widetilde{B}=\mathbb{C P}^{1}$ have stabilizer $\mathbb{Z}_{2}$ in each case, so on the quotient $Z / G$ we will find fibers with multiplicity 2 which become $I_{0}^{*}$ Kodaira fibers on $\widetilde{Z / G}$. Once again the lattices satisfy $L=\Gamma_{1,1}(2) \oplus L_{x}$.

Turning to the case in which $Z=T^{4}$, we find a similar story. The base $B$ of the elliptic fibration on $Z / G$ is still $\mathbb{C P}^{1}$, but the base $\widetilde{B}$ of the elliptic 
fibration on $Z$ is now an elliptic curve. The action of $G$ on $\widetilde{B}$ must be faithful, and in fact it suffices to consider the case in which $G$ does not contain any translations (else we could mod out by the translations first). As an action on an elliptic curve with a fixed point, the only possibilities are $G=\mathbb{Z}_{m}$ with $m=2,3,4$, or 6 . In fact, these are exactly the group actions that we have (in table 10)! As in the previous case, the singular points on $Z / G$ can be collected into Kodaira fibers for $\widetilde{Z / G}$; the results of this are displayed in table 13. The lattices once again satisfy $L \cong \Gamma_{1,1}(m) \oplus L_{x}$.

The entries in tables 11, 12, and 13 thus describe the possible $\mathrm{F}$ theory vacua with 16 supercharges. The first entry in table 11 gives the "standard" F theory vacuum in eight dimensions, the remaining five entries in table 11 together with the four entries in table 13 give the new $\mathrm{F}$ theory vacua in seven dimensions, and table 12 shows the one new $\mathrm{F}$ theory vacuum in six dimensions. (Of course the higher dimensional theories can be reduced to lower dimensional ones by compactification on additional circles.)

\subsubsection{From $\mathrm{F}$ theory to type $\mathrm{I}^{\prime}$}

Decompactification limits of the components of the $\mathrm{F}$ theory moduli space which have $D$-dimensional effective theories should lead to $(D+1)$-dimensional effective theories (using an isotropic sublattice of rank 1). It would be desirable to give a description of these directly in terms of type $\mathrm{I}^{\prime}$ theory. However, despite the interesting pictures presented in [70,71], at present we do not have enough control over type $\mathrm{I}^{\prime}$ vacua to be able to do this. So our analysis will be somewhat indirect. In this section, we describe the overall picture leaving a detailed discussion for the following section.

The most common decompactification limit from these components involves a decomposition of the lattice in the form

$$
L \cong \Gamma_{1,1} \oplus L_{x}
$$

We saw in the previous section that when going from six dimensions up 
to seven dimensions, a lattice decomposition of this form corresponded to a boundary component which gained additional degrees of freedom in the limit, and which "attached" to the standard boundary component.

To see this, we will give a geometric construction of these components and their decompactification limits, and note that for the vast majority of components in dimension seven, there is only one decompactification limit, which must therefore be of this type. We thus argue by analogy that all limits of this type must have the lattice decomposition given in eq. (74); this leaves only a few additional components in dimension eight for which we must account.

Our geometric construction-to be described in section 4.3 .2 for $Z=$ $K 3$-is designed for easy comparison with the heterotic duals of these vacua, where this phenomenon is known: the seven-dimensional components we have found are dual to non-trivial triples of Wilson lines on the heterotic side, and their eight-dimensional limits all attach to the standard eightdimensional component. The exceptional case is $G=\mathbb{Z}_{2}$ which contains the CHL string. This case does have a non-trivial 8 and 9-dimensional limit.

A similar discussion applies to the 8-dimensional limits of the $\mathrm{F}$ theory moduli spaces coming from $Y=\left(S^{1} \times T^{4}\right) / G$. Since the lattices for these models also have a $\Gamma_{1,1}$ summand, 8-dimensional limits exist. However, in each case, again with the exception of $G=\mathbb{Z}_{2}$, these boundaries attach to a conventional toroidal type IIB compactification. The case of $G=\mathbb{Z}_{2}$ has non-trivial 8 and 9-dimensional limits as reflected in its lattice given in table 11. This is natural since this model has a dual description as the compactification of the $(+,-)$ orientifold to 7 dimensions. This duality can be seen immediately by studying the geometric description of the compactified $(+,-)$ orientifold obtained in section 3.2.6.

The last case we need to discuss is the 6 -dimensional $\mathrm{F}$ theory vacuum associated to $G=\mathbb{Z}_{2} \times \mathbb{Z}_{2}$. From the lattice for $\widetilde{K 3 / G}$ given in table 12 , we that there are two ways of decompactifying to 8 dimensions. Peeling off 
a $\Gamma_{1,1}$ factor gives us a 7 -dimensional theory that attaches to the standard component as in our preceeding discussion. The other limit involves peeling off a $\Gamma_{1,1}(2)$ factor. This leads to a 7-dimensional theory that attaches to the component of the moduli space containing the CHL string. Note that we also need to worry about the additional signature $(1,1)$ summand in the lattice for $\mathrm{F}$ theory on $\left(K 3 \times T^{2}\right) / G$. The correct relative normalization for this summand is tricky to determine, although preliminary computations suggest that it is either $\Gamma_{1,1}$ or $\Gamma_{1,1}(2)$. This is certainly supported from a study of the $\mathbb{Z}_{2} \times \mathbb{Z}_{2}$ asymmetric orbifold of the heterotic string. From that approach, it seems clear that there is no new 7-dimensional theory to which the 6dimensional orbifold could decompactify. From this 6-dimensional theory, we therefore arrive at no new 7-dimensional theory.

\subsubsection{Automorphisms of del Pezzo surfaces}

Let us begin by explaining why del Pezzo surfaces play a natural role in our $\mathrm{F}$ theory discussion. Consider an elliptically fibered $K 3$ surface $Z$ whose base $B$ is large, and whose complex structure is close to a degenerate one in which the $K 3$ surface degenerates into a pair of del Pezzo surfaces $Z_{1}, Z_{2}$ meeting on an elliptic curve $E$. This is the limit in which comparison with the $E_{8} \times E_{8}$ heterotic string becomes possible [72]. (In fact, it corresponds to both large volume and weak string coupling on the heterotic side of the duality.) It is then natural to expect that automorphisms of del Pezzo surfaces will be classified by the same data used to classify $E_{8}$ gauge bundles on $T^{3}$. Further, the anomaly cancellation condition should have a purely geometric realization as a constraint on whether we can "glue" two del Pezzo surfaces with automorphisms into a $K 3$.

So let us begin by considering a rational elliptic surface $X$ in Weierstrass form. This implies that $X$ has an elliptic fibration $\pi: X \rightarrow \mathbb{C P}^{1}$, and a section $\sigma: \mathbb{C P}^{1} \rightarrow X$ contained in the smooth points of $X$. Since $X$ is rational, $\sigma^{2}=-1$, that is to say $\sigma$ is an exceptional curve. We fix a fiber 
$E \subset X$ of $\pi$, and we wish to study the group of automorphisms of $X$ which are the identity on $E$ and which stabilize $\sigma$ (ie map the image of $\sigma$ to itself). We call these automorphisms of $(X, E, \sigma)$ Equivalently, we could consider a degree one del Pezzo surface $\bar{X}$ obtained by collapsing $\sigma$. The elliptic fibration on $X$ becomes the anti-canonical pencil of elliptic curves (or more generally Weierstrass curves) on this surface. This surface has at worst rational double point singularities. The automorphism group $(X, E, \sigma)$ acts naturally on $\bar{X}$ and is identified with the subgroup of the automorphism group of $X$ fixing $E$ pointwise.

As a first step, let us argue that the automorphism group of $(X, E, \sigma)$ is a finite cyclic group that preserves the elliptic fibration structure of $X$. Along the way, we shall also see that the induced action of the group of automorphisms on the base $\mathbb{C P}^{1}$ of the elliptic fibration is faithful. The group acts on $\mathbb{C P}^{1}$ fixing two points, i.e., the automorphism group of $(X, E, \sigma)$ stabilizes $E$ and exactly one other fiber.

To see this, let $f \subset X$ be a fiber of the elliptic fibration and let $\alpha: X \rightarrow$ $X$ be an automorphism of $(X, E, \sigma)$. Then $\alpha(f)$ is a divisor in $X$ with zero algebraic intersection with $E$. Hence, its projection to $\mathbb{C P}^{1}$ must be a single point. That is to say $\alpha(f)$ is contained in a fiber of the elliptic fibration. By homological considerations, we see that it is exactly a fiber of the elliptic fibration structure. This shows that $\alpha$ preserves the elliptic fibration structure and hence induces an automorphism $\bar{\alpha}$ of the base $\mathbb{C P}^{1}$. If $\bar{\alpha}$ is trivial, then $\alpha$ stablizes each fiber of the elliptic fibration. Since it acts by the identity on $E$, it acts by the identity on the homology of each smooth fiber and hence, it is a translation on each smooth fiber. But, $\alpha$ also stablizes the section $\sigma$, and hence it must be the identity on each smooth fiber. Since the smooth fibers are dense, it follows that $\alpha$ is the identity. This proves that the automorphism group of $(X, E, \sigma)$ acts faithfully on the base $\mathbb{C P}^{1}$.

The elliptic fibration structure on $X$ has at least two singular fibers. This 
means that the automorphism group of $(X, E, \sigma)$ is faithfully represented as a group of automorphisms of $\mathbb{C P}^{1}$ fixing a point of $\mathbb{C P}^{1}$ and permuting a finite set of points of cardinality at least two. All such groups are finite cyclic and fix two points of $\mathbb{C P}^{1}$.

We use $E^{\prime}$ to denote the fiber other than $E$ stabilized by the automorphism group of $(X, E, \sigma)$. Suppose that an automorphism $\alpha$ is the identity on $E^{\prime}$. It is also the identity on $E$. We consider the degree one del Pezzo model $\bar{X}$ where the section has been collapsed. The image of $\sigma$ is a smooth point of this surface, and $\alpha$ descends to an automorphism $\bar{\alpha}$ of $\bar{X}$ fixing the point of intersection $x$ of $E$ and $E^{\prime}$ and acting by the identity on $E$ and on $E^{\prime}$. If follows that the differential of $\bar{\alpha}$ at $x$ is the identity, and hence that the restriction of $\alpha$ to the exceptional curve in $X$ obtained from blowing up $x$ is the identity. It follows that $\alpha$ stabilizes each fiber of the elliptic fibration. But we have already seen that this implies that $\alpha$ is the identity. The action of the automorphism group of $(X, E, \sigma)$ on $E^{\prime}$ is therefore faithful.

Let us examine the automorphism groups of the various types of Weierstrass curves fixing a given smooth point of the curve. The automorphism group of a generic elliptic curve fixing a point is $\mathbb{Z}_{2}$ acting by -1 and fixing 4 points. In the case of special elliptic curves the automorphism group is either $\mathbb{Z}_{4}$ acting with two fixed points and two points with stabilizers $\mathbb{Z}_{2}$, or $\mathbb{Z}_{6}$ acting with one fixed point, 3 points with stabilizer $\mathbb{Z}_{2}$ and two points with stabilizer $\mathbb{Z}_{3}$. The automorphism group of an ordinary double point fiber fixing a smooth point is $\mathbb{Z}_{2}$ fixing the singular point and the other point. The automorphism group of a Weierstrass cusp fixing a smooth point is $\mathbb{C}^{*}$ acting so as to fix only the singular point and the given smooth point.

Let $Y$ be an elliptic surface and let $f \subset Y$ be a fiber. Let $p \in \mathbb{C P}^{1}$ be the image of $f$ under projection mapping. Let $\tilde{Y} \rightarrow Y$ be the minimal resolution of $Y$ and let $\tilde{p}: \tilde{Y} \rightarrow \mathbb{C P}^{1}$ be the induced projection mapping. Let $\tilde{f} \subset \tilde{Y}$ be the full preimage of $f$. Fix a disk $\Delta \subset \mathbb{C P}^{1}$ centered at $p$ sufficiently small so that the preimage of $\Delta-\{p\}$ contains the preimage of 
no singular point of $Y$ or of the projection mapping. We define the local contribution of $f$ to the Euler characteristic of the smooth model of $Y$ to be the Euler characteristic of $\widetilde{p}^{-1}(\Delta)$. It is easy to see that $f$ is a smooth fiber if and only if its local contribution to the Euler characteristic is zero and otherwise that the local contribution to the Euler characteristic is positive. Also, the local contribution to the Euler characteristic is 1 if and only if $f$ misses the singularities of $X$ and contains an ordinary double point. Lastly, if the elliptic surface in question is rational, then the sum over all fibers of the local contributions to the Euler characteristic is 12 .

The quotient of $X$ by the automorphism group is a surface $Y$ with an induced elliptic fibration and with rational double point singularities. In the singular model, the fiber which contains the image of $E^{\prime}$ has multiplicity equal to the order of the automorphism group of $X$. On the other hand, in the minimal resolution, the strict transform of this curve is one of the components of the set of rational curves indexed by the nodes of an extended Dynkin diagram of type $A, D$, or $E$ and which intersect each other as indicated by the bonds of the extended Dynkin diagram. Furthermore, the multiplicities of the various components in the fiber in the smooth model are given by the coroot integers on the corresponding nodes of this diagram. These numbers are all at most 6 , and hence every component has multiplicity at most 6 in the fiber. It follows in the singular model, that the fiber containing the image of $E^{\prime}$ has multiplicity at most 6 , and hence the order of the automorphism group is at most 6 . (Notice that the cases of smooth and nodal fibers $E^{\prime}$ follow directly from the classification of the automorphism groups of these fibers given above.) The automorphism group of $(X, E, \sigma)$ is therefore a cyclic group of order at most 6 .

Now we simply list the possibilities: the automorphism group is $\mathbb{Z}_{2}$ and the fiber $E^{\prime}$ stabilized by the action is smooth. The quotient surface has four $A_{1}$ singularities and the image of $E^{\prime}$ passes through all of these. The local contribution of this fiber to the Euler characteristic of the smooth model is 6. The local contribution to the Euler characteristic of the smooth model 
of $X$ from the singular fibers of the elliptic fibration is 12 , and since the automorphism group acts freely on these fibers, the local contribution of the images of these fibers to the Euler characteristic of the smooth model of the quotient is $12 / 2=6$. In the minimal resolution of the quotient surface, the preimage of this fiber is a tree of rational curves intersecting according to the extended Dynkin diagram of $D_{4}$. The strict transform of $E^{\prime}$ has multiplicity 2 in the fiber, i.e., it corresponds to the central node in the extended Dynkin diagram, the one with coefficient two in the dominant root.

The automorphism group is $\mathbb{Z}_{3}$ and the fiber $E^{\prime}$ stabilized by the action is smooth. The quotient surface has three $A_{2}$ singularities and the image of $E^{\prime}$ passes through all of them. In the minimal resolution of the quotient surface, the preimage of this fiber is a tree of rational curves intersecting according to the extended Dynkin diagram of $E_{6}$ and this fiber contributes 8 to the Euler characteristic of the smooth model. The singular fibers contribute 12 to the Euler characteristic of the smooth model of $X$ and hence their images in the quotient contribute $12 / 3=4$ to the Euler characteristic of its smooth model. The strict transform of $E^{\prime}$ is the curve of multiplicity three in the fiber, i.e., it corresponds to the central node in the extended Dynkin diagram, the one with coefficient three in the dominant root. The local contribution of this fiber to the Euler characteristic of the smooth model is 8 .

The automorphism group is $\mathbb{Z}_{4}$ and the fiber $E^{\prime}$ stabilized by the action is smooth. The quotient surface has two $A_{3}$ singularities and an $A_{1}$ singularity. The image of $E^{\prime}$ passes through all these singularities. In the minimal resolution of the quotient surface the preimage of this fiber is a tree of rational curves intersecting according to the extended Dynkin diagram of $E_{7}$ and the local contribution of this fiber to the Euler characteristic of the smooth model is 9 . The singular fibers contribute 12 to the Euler characteristic of the smooth model of $X$, and hence the images in the quotient of the singular fibers of $X$ contribute $12 / 4=3$ to the Euler characteristic of the smooth model of the quotient. The strict transform of $E^{\prime}$ is the curve of multiplicity 4 in the fiber, i.e., corresponds to the node with coefficient 4 in the dominant 
root.

The automorphism group is $\mathbb{Z}_{5}$ and the fiber $E^{\prime}$ is a Weierstrass cusp. The quotient surface has two $A_{4}$ singularities and the image of $E^{\prime}$ passes through both of them. The preimage of this fiber in the minimal resolution of the quotient is a tree of rational curves intersecting according to the extended Dynkin diagram of $E_{8}$ and contributes 10 to the Euler characteristic of the smooth model of the quotient. The other singular fibers of $X$ contribute 10 to the Euler characteristic of the smooth model of $X$ and their images in the quotient contribute $10 / 5=2$ to the Euler characteristic of its smooth model. The strict transform of $E^{\prime}$ is the curve in this configuration with multiplicity 5 in the divisor representing the fiber. That is to say it corresponds to the node with coefficient 5 in the dominant root. The local contribution of this fiber to the Euler characteristic of the smooth model is 10. Notice that since $E^{\prime}$ is a cusp, the sum of the contributions of the all other fibers to the Euler characteristic of the smooth model of $X$ is 10 . Thus, in the quotient the sum of the local contributions of the fibers besides the image of $E^{\prime}$ to the Euler characteristic of the smooth model is 2 , giving us a total of 12 as required.

The automorphism group is $\mathbb{Z}_{6}$ and the fiber $E^{\prime}$ is smooth. The quotient surface has three singularities-of types $A_{5}, A_{2}$ and $A_{1}$, respectively, reflecting the three singular orbits of the action of this cyclic group on a smooth elliptic curve. The image of $E^{\prime}$ passes through all these singularities and its preimage in the minimal resolution is a tree of rational curves intersecting according to the extended Dynkin diagram of $E_{8}$. The strict transform of $E^{\prime}$ has multiplicity 6 in the fiber and hence corresponds to the trivalent node in the extended Dynkin diagram, the one with coefficient 6 in the dominant root. The local contribution of this fiber to the Euler characteristic of the smooth model is 10 . The singular fibers of $X$ contribute 12 to the Euler characteristic of its smooth model, and the images of these fibers in the quotient contribute $12 / 6=2$ to the Euler characteristic of its smooth model.

This completes the list of possibilities. Notice how the extended Dynkin 
diagram of $E_{8}$ predicts the automorphism groups and the singularities of the quotient surfaces. First of all there will be an automorphism group of order $k$ if and only if one of the coefficients on the extended Dynkin diagram of $E_{8}$ is divisible by $k$. Given an integer $k$ with this property, the singularity in the quotient surface when the automorphism group is cyclic of order $k$ is determined as follows: One takes the extended Dynkin diagram of $E_{8}$ with the usual coefficients and removes all nodes whose coefficients are divisible by $k$. There remains a collection of $A_{n_{i}}$ diagrams. These label the singularities of the quotient surface. Furthermore, we can connect all of these diagrams to a central node and form an extended Dynkin diagram of some subgroup of $E_{8}$. In that new diagram the coefficient of the central node that we added will be exactly $k$. This is the fiber in the minimal resolution of the quotient. The strict transform of the image of $E^{\prime}$ is the component corresponding to the central node that we added.

Note that the list of possibilities matches perfectly with the gauge theory picture of commuting pairs in $E_{8}$ and their centralisers. Of course, this is not an accident: one can prove by abstract methods that equivalence between the group theory and del Pezzo surfaces which goes through the Looijenga space of $E \otimes \Lambda\left(E_{8}\right) / W\left(E_{8}\right)$ is categorical and hence that the automorphism groups of the three classes of objects-commuting pairs in $E_{8}, E \otimes \Lambda\left(E_{8}\right) / W\left(E_{8}\right)$ (here, $\Lambda\left(E_{8}\right)$ is the coroot lattice of $E_{8}$ and $W\left(E_{8}\right)$ is its Weyl group) and del Pezzo surfaces must be the same. ${ }^{18}$

One last point is worth remarking on: given two rational elliptic surfaces with smooth fibers and sections $\left(X_{1}, E_{1}, \sigma_{1}\right)$ and $\left(X_{2}, E_{2}, \sigma_{2}\right)$ and given an isomorphism from $E_{1}$ to $E_{2}$ matching up the intersections with the sections, we can glue $X_{1}$ and $X_{2}$ together to form a singular surface $X$ with a normal crossing along $E=E_{1}=E_{2}$. This surface fibers over $\mathbb{C P}^{1} \cup \mathbb{C P}^{1}$ with a section $\sigma$. Of course, it has a marked fiber $E$. It is a singular model of an elliptically fibered $K 3$ surface, and in fact represents a point in a divisor

\footnotetext{
${ }^{18}$ We wish to thank Bob Friedman for pointing this out to us.
} 
at infinity in a compactification of period space for these surfaces. ${ }^{19}$ Given automorphisms $\alpha_{i}$ of $\left(X_{i}, E_{i}, \sigma_{i}\right)$ we can glue them together to determine an automorphism of $(X, E, \sigma)$. To smooth the singular surface $X$ to an elliptically fibered $K 3$ we need a trivialization of the tensor product of the normal bundles of $E$ in $X_{1}$ and $X_{2}$. To carry along the group action requires then trivializing the action of this tensor product. This is possible if and only if the actions of $\alpha_{i}$ on the disks in the base $\mathbb{C P}^{1}$ 's centered at the image points of $E_{i}$ are inverses of each other. In particular, the orders of $\alpha_{1}$ and $\alpha_{2}$ must be the same and these two automorphisms must be in inverse components. This corresponds in the gauge theory language to the fact that the ChernSimons invariant of the commuting triples must be inverses of each other. As promised, we therefore recover our anomaly matching constraint from this gluing condition.

\subsection{Type IIA compactifications with RR one-form flux}

\subsubsection{Equivariant flat line bundles on $T^{4}$}

We now take a different tack and consider compactifications with flux. This is a quite different class of models from the purely geometric compactifications just discussed. Among compactifications of this kind, we shall find new dual descriptions for the perturbative asymmetric orbifolds of section 2 both in 6 and 7 dimensions.

Let us first consider flat RR 1-form fields in a type IIA string compactification on some (possibly singular) manifold $X$. Such a 1-form field $A$ can be seen as a connection in a principal $U(1)$ bundle $P \rightarrow X$. Of course the interpretation in $\mathrm{M}$ theory of such a RR 1-form field configuration will be as a compactification on the manifold $P$. In our preceeding discussion, $P$ took the form of $\left(X \times S^{1}\right) / G$. As we shall see, it is natural from this purely geometric $\mathrm{M}$ theory picture to treat $A$ via equivariant cohomology. In section 4.5 , we revisit this treatment from the perspective of K-theory where we find

\footnotetext{
${ }^{19}$ On the heterotic side, this represents the infinite-volume, zero-coupling limit.
} 
a group of 1-form fluxes in agreement with the results from equivariant cohomology. K-theory, however, will give us new physics for compactifications with more general combinations of fluxes.

$U(1)$ principal bundles, or equivalently complex Hermitian line bundles, over a smooth manifold $X$ are classified topologically by their first Chern class $c_{1}$, which is an element of the cohomology group $H^{2}(X, \mathbb{Z})$. This Chern class equals $[F / 2 \pi]$ in real cohomology, where $F=d A$ is the curvature twoform. Flat line bundles, i.e., bundles that satisfies $F=0$, are classified by the cohomology group $H^{1}(X, U(1))$. One can think of this group of homomorphisms of $H_{1}(X, \mathbb{Z})$ into $U(1)$ as the holonomies of the flat connection around the non-trivial homology 1-cycles of $X$. Note that because $F$ vanishes, in general such a flat bundle can have only have a torsion first Chern class

$$
c_{1} \in \operatorname{Tor} H^{2}(X, \mathbb{Z})
$$

If there is no torsion in $H^{2}(X)$ then a flat line bundle is necessarily topologically trivial. It can still have non-trivial holonomies. In fact the group $H^{1}(X, U(1))$ of flat line bundles fits in the exact sequence

$$
1 \rightarrow H^{1}(X, \mathbb{R}) / H^{1}(X, \mathbb{Z}) \rightarrow H^{1}(X, U(1)) \rightarrow \text { Tor } H^{2}(X, \mathbb{Z}) \rightarrow 1
$$

where the Jacobian torus

$$
H^{1}(X, \mathbb{R}) / H^{1}(X, \mathbb{Z})
$$

gives the holonomies of flat connections on bundles that are topologically trivial.

As explained in appendix $\mathrm{D}$, for the case of an orbifold $X / G$, we have to consider equivariant line bundles on $X$. These are bundles over $X$ with a compatible $G$ action. Equivariant flat line bundles are classified by the equivariant cohomology group

$$
H_{G}^{1}(X, U(1))
$$


This will be the group of RR 1-form fluxes.

We will be considering the case where $X$ is either a $T^{4}$ or a $K 3$ manifold. In these cases, there is a well-known list of orbifold groups $G$ such that $X / G$ is again Calabi-Yau, i.e., a K3 manifold.

For the case $X=T^{4}$, the groups are listed in [56]

$$
G=\mathbb{Z}_{2}, \mathbb{Z}_{3}, \mathbb{Z}_{4}, \mathbb{Z}_{6}, \widehat{\mathcal{D}}_{4}, \widehat{\mathcal{D}}_{5}, \mathbb{T} .
$$

Here $\widehat{\mathcal{D}}_{N}$ denotes the binary dihedral group of order $4 N$, a double cover of the ordinary dihedral group $\mathcal{D}_{N}$. In the $A D E$-labeling of finite subgroups of $S U(2)$, this group corresponds to the Dynkin diagram $D_{N+2}$, just as the cyclic group $\mathbb{Z}_{N}$ corresponds to $A_{N-1}$. The last case $\mathbb{T}$ is the binary tetrahedral group, which corresponds to $E_{6}$. For a list of the group actions along with the singularities of $T^{4} / G$, see table 18 of appendix D.

To be concrete let us first consider this group for the case of the $\mathbb{Z}_{2}$ action $x \rightarrow-x$ on the torus $T^{4}$. Here the group $H_{\mathbb{Z}_{2}}^{*}\left(T^{4}, U(1)\right)$ can be computed by a spectral sequence technique ${ }^{20}$ which is given in appendix $\mathrm{D}$.

This calculation shows that it essentially suffices to compute the equivariant cohomology with coefficients in the field $\mathbb{Z}_{2}$ which has a rather simple description. Is this case there are no differentials in the $E_{2}$-term of the spectral sequence and no extension problems so that

$$
H_{\mathbb{Z}_{2}}^{k}\left(T^{4}, \mathbb{Z}_{2}\right)=\bigoplus_{p+q=k} H^{p}\left(\mathbb{R} \mathbb{P}^{\infty}, H^{q}\left(T^{4}, \mathbb{Z}_{2}\right)\right)
$$

The cohomology of $T^{4}$ with $\mathbb{Z}_{2}$ coefficients is given by generators $\theta^{i}$ of degree 1 satisfying $\left(\theta^{i}\right)^{2}=0$. The $\theta^{i}$ can be thought of as the mod 2 reductions of the one-forms $d x^{i}$. Because we work with $\mathbb{Z}_{2}$ coefficients, they are invariant under the orbifold group, since the group acts as $\theta^{i} \rightarrow-\theta^{i}=\theta^{i}$. In the equivariant cohomology of $T^{4}$, we have to add an extra generator $\xi$ also of degree one. The element $\xi$ is the generator of the group cohomology $H^{*}\left(B \mathbb{Z}_{2}, \mathbb{Z}_{2}\right)$.

\footnotetext{
${ }^{20}$ We thank D. Freed and G. Segal for help with these calculations.
} 
There is subtle point in that the relation among the generators $\theta^{i}$ is modified to

$$
\theta^{i}\left(\xi-\theta^{i}\right)=0
$$

This relation replaces the relation $\left(\theta^{i}\right)^{2}=0$ in the cohomology of $T^{4}$. It has a simple geometric interpretation that becomes already clear if we consider the case of $S^{1}$. Since we work with $\mathbb{Z}_{2}$ coefficients, let us consider a real line bundle over the orbifold $S^{1} / \mathbb{Z}_{2}$. Upstairs, we have a bundle over $S^{1}$ and this bundle is either trivial or the Möbius bundle. The $\mathbb{Z}_{2}$ action on $S^{1}$ has two fixed points at $x=0$ and $x=\pi$. We consider this bundle locally around one of the fixed point, say $x=0$. Let $y$ be the coordinate in the fiber. The group action will map $x \rightarrow-x$ and for the fiber coordinate we have two possibilities: either the trivial action $y \rightarrow y$ or the non-trivial action $y \rightarrow-y$. Since we have two fixed points this gives a total of $2^{2}=4$ possibilities.

These 4 possibilities have a clear interpretation as equivariant bundles on $S^{1}$. If the group action is trivial at both fixed points we obtain the trivial bundle on $S^{1}$ with the trivial group action. If the group action is only non-trivial at one fixed point, we obtain the Möbius bundle with the two possible $\mathbb{Z}_{2}$ actions. Finally if the group action is non-trivial at both fixed points, the corresponding bundle on $S^{1}$ is trivial, but it has a non-trivial group action that can globally be written as $y \rightarrow-y$. This represents the equivariant bundle that we denoted as $\xi$. So, if $\theta_{0}$ and $\theta_{1}$ denote the classes that represent the non-trivial bundles around the fixed points $x=0$ and $x=\pi$, then we clearly have the relation

$$
\theta_{0}+\theta_{1}=\xi
$$

Now the $\mathbb{Z}_{2}$-equivariant cohomology of $S^{1}$ is very simple. As explained in appendix D, it is the cohomology of the homotopy quotient $S_{\mathbb{Z}_{2}}^{1}$ which is a circle bundle over the classifying space $\mathbb{R} P P^{\infty}$. We can think of $S^{1}$ as the union of two line intervals, glued together at $x=0$ and $x=\pi$. The corresponding bundles of these intervals over $\mathbb{R}^{\infty}$ have contractible fibers 
and so are homotopic to $\mathbb{R P}^{\infty}$ itself. The intersection of the two intervals gives rise to a $\mathbb{Z}_{2}$ bundle over $\mathbb{R P}^{\infty}$ with a total space that, by definition, is contractibe to a point. Therefore $S_{\mathbb{Z}_{2}}^{1}$ is homotopically a wedge $\mathbb{R} \mathbb{P}^{\infty} \vee \mathbb{R}^{\infty}$, i.e., two copies of $\mathbb{R P}^{\infty}$ glued together at a point. The classes $\theta_{0}$ and $\theta_{1}$ are the generators of the two copies of $H^{*}\left(\mathbb{R}^{P^{\infty}}, \mathbb{Z}_{2}\right)$, and clearly we have

$$
\theta_{0} \cup \theta_{1}=0
$$

So the equivariant cohomology of $S^{1}$ is given by the ring

$$
H_{\mathbb{Z}_{2}}^{*}\left(S^{1}, \mathbb{Z}_{2}\right) \cong \mathbb{Z}_{2}\left[\theta_{0}, \theta_{1}\right] /\left(\theta_{0} \theta_{1}\right)
$$

Both the elements $\theta_{0}$ and $\theta_{1}$ map to the invariant element in $H^{1}\left(S^{1}, \mathbb{Z}_{2}\right)$ that represents the Möbius bundle. We can pick as generators $\theta:=\theta_{1}$ and $\xi$. But we should impose the relation

$$
\theta(\xi-\theta)=0
$$

Note that the translation $S^{1}$ over a half-period that interchanges the fixed points is represented by $\theta \rightarrow \theta+\xi$ which indeed interchanges $\theta_{0}$ and $\theta_{1}$.

Generalizing this construction to the 4-torus gives the relation (81) among the $\theta^{i}$ and $\xi$. In that case a translation $x^{i} \rightarrow x^{i}+\pi q^{i}, q^{i} \in \mathbb{Z}_{2}$ acts on the cohomology generators as

$$
\theta^{i} \rightarrow \theta^{i}+q^{i} \xi
$$

A direct computation shows that all classes in $H_{\mathbb{Z}_{2}}^{1}\left(T^{4}, \mathbb{Z}_{2}\right)$ correspond to classes with $U(1)$ coefficients, so that we find that

$$
H_{\mathbb{Z}_{2}}^{1}\left(T^{4}, U(1)\right) \cong \mathbb{Z}_{2}^{5}
$$

We can pick a basis given by $\theta^{1}, \theta^{2}, \theta^{3}, \theta^{4}, \xi$ and write a general equivariant flat bundle as

$$
A=\sum_{i=1}^{4} a_{i} \theta^{i}+a_{0} \xi, \quad a_{i}, a_{0} \in \mathbb{Z}_{2} .
$$


Here the coefficients $a_{i}, a_{0}$ take value in the field of two elements that we simply write as $\mathbb{Z}_{2}$, i.e., the set $\{0,1\}$ with addition and multiplication modulo (which is often written as $\mathbb{F}_{2}$ ). So altogether we have $2^{5}=32$ equivariant flat bundles on $T^{4}$.

We can understand this result directly in terms of bundles on $T^{4}$. We first pick a flat connection $A=A_{i} d x^{i}$ on $T^{4}$. Such a connection has holonomy

$$
\exp \oint_{\gamma_{i}} A=\exp 2 \pi i A_{i}
$$

around the one-cycle $\gamma_{i}$ along the $x^{i}$ axis. The holonomy takes value in the torus

$$
H^{1}\left(T^{4}, U(1)\right) \cong\left(T^{4}\right)^{*} .
$$

The orbifold group acts on the flat gauge field by $A_{i} \rightarrow-A_{i}$, where we identify $A_{i} \cong A_{i}+1$. So the invariant connections are given by

$$
A_{i}=0,1 / 2
$$

which are the points of order two on the Jacobian. This gives a total of $2^{4}=16$ invariant bundles. Now we have to decide how the group $\mathbb{Z}_{2}$ acts on the fiber of the line bundle. Here we can have either the trivial action or the non-trivial action. This multiplies the total number of bundles by two with a total of 32 bundles.

In terms of the equivariant cohomology classes that we described above we have the following interpretation of these 32 bundles. The element $\xi$ clearly represents the trivial flat bundle on $T^{4}$ with the non-trivial group action. It corresponds to the non-trivial element in the group cohomology $H^{1}\left(B \mathbb{Z}_{2}, U(1)\right)$.

Let us consider the corresponding $M$ theory compactifications. First we remark that these 32 cases decompose into orbits of $S L\left(4, \mathbb{Z}_{2}\right)$. In particular the 16 invariant flat connections $A=a_{i} \theta^{i}$ behave very much as spin structures on a two-torus. They form two orbits: a singlet of trivial holonomy $A=0$ (the "odd spin structure") and a remaining orbit of 15 non-trivial 
holonomies $A=a_{i} \theta^{i}$ with not all $a_{i}=0$ (the "even spin structures"). (Here 15 is the adjoint representation of $S L\left(4, \mathbb{Z}_{2}\right)$.) A similar decomposition of $16=1+15$ holds for the bundles of type $A=a_{i} \theta^{i}+\xi$. Both $a_{i} \theta^{i}$ and $a_{i} \theta^{i}+\xi$ map to the flat connection $A_{i}=\frac{1}{2} a_{i}$.

Note that a half shift along the torus can interchange the two $\mathbf{1 5}$ orbits. In fact the full symmetry group is

$$
\left(\mathbb{Z}_{2}\right)^{4} \ltimes S L\left(4, \mathbb{Z}_{2}\right)
$$

which is the affine group acting on $\left(\mathbb{Z}_{2}\right)^{4}$. We can think of the expression $\left(a_{i}, a_{0}\right)$ as defining the affine linear function $a_{i} x^{i}+a_{0}$. If not all $a_{i}$ vanish this defines one of the 30 affine planes. This gives the orbit $\mathbf{3 0}$. The two constant functions are singlets.

This split $\mathbf{3 2}=\mathbf{1}+\mathbf{1}+\mathbf{3 0}$ also manifests itself geometrically in the $\mathrm{M}$ theory interpretations. The singlet $A=0$, of course, corresponds to the trivial compactification $T^{4} \times S^{1} \cong T^{5}$. The other singlet $A=\xi$ gives a compactification manifold

$$
\left(T^{4} \times S^{1}\right) / \mathbb{Z}_{2}
$$

where the $\mathbb{Z}_{2}$ acts as a shift $x^{11} \rightarrow x^{11}+\pi$. The action is fixed point free. This manifold breaks half the supersymmetries. The moduli are simply the NS fields (metric and $B$-field) on $T^{5}$ plus the string coupling constant (radius $S^{1}$ ). There are no continuous RR field moduli. That is, this compactification has exactly the same number of moduli as the CFT on $T^{4}$.

All of the other bundles can also be obtained as quotients of $T^{5}$, now considered as the trivial circle bundle over a double cover $\widehat{T^{4}}$ of the original IIA 4-torus $T^{4}$. First, by an $S L\left(4, \mathbb{Z}_{2}\right)$ transformation we can map $A$ to the form $A=\theta^{1}$ or $A=\theta^{1}+\xi$. In the case $A=\theta^{1}$ we define the cover $\widehat{T^{4}}$ by making the coordinate $x^{1}$ periodic modulo $4 \pi$. We then obtain the bundle by the quotient

$$
\left(\widehat{T^{4}} \times S^{1}\right) / \mathbb{Z}_{2} \times \mathbb{Z}_{2}
$$


Here the first $\mathbb{Z}_{2}$ (the deck transformation of the cover) acts as

$$
g_{1}: x^{1} \rightarrow x^{1}+2 \pi, \quad x^{11} \rightarrow x^{11}+\pi
$$

while the second $\mathbb{Z}_{2}$ (the original orbifold group) acts as

$$
g_{2}: x^{i} \rightarrow-x^{i}, \quad i=1, \ldots, 4 .
$$

If we first divide out $g_{2}$, we obtain a description as the quotient

$$
\left(K 3 \times S^{1}\right) / \mathbb{Z}_{2}
$$

where $K 3$ is the Kummer surfaces $\widehat{T^{4}} / \mathbb{Z}_{2}$ and the $\mathbb{Z}_{2}$ is the involution that exchanges the 16 fixed points $x^{i}=0,2 \pi$ in pairs.

Similar remarks hold for the case $A=\theta^{1}+\xi$. Here we find a $\mathbb{Z}_{2} \times \mathbb{Z}_{2}$ action generated by

$$
g_{1}: x^{1} \rightarrow x^{1}+2 \pi, \quad x^{11} \rightarrow x^{11}+\pi
$$

and

$$
g_{2}: x^{i} \rightarrow-x^{i}, \quad x^{11} \rightarrow x^{11}+\pi .
$$

In this case it makes sense to first quotient by,

$$
g_{1} g_{2}: x^{i} \rightarrow 2 \pi-x^{i}
$$

This again produces a singular $K 3$ manifold, now with fixed points at $x^{i}=$ $\pi, 3 \pi$. This is no surprise because we already remarked that a half shift interchanges these two types of compactification.

In summary, the $\mathrm{M}$ theory geometry of a type IIA orbifold $T^{4} / \mathbb{Z}_{2}$ with possible 1-form flux is one of the following three forms:

$$
T^{5},\left(T^{4} \times S^{1}\right) / \mathbb{Z}_{2},\left(T^{4} / \mathbb{Z}_{2} \times S^{1}\right) / \mathbb{Z}_{2}
$$

reflecting the $\mathbf{3 2}=\mathbf{1}+\mathbf{1}+\mathbf{3 0}$ decomposition of $\left(\mathbb{Z}_{2}\right)^{5}$. The extension to more general quotients is discussed in appendix D.2. 


\subsubsection{Local holonomies}

We now turn to the local description of these bundles on the orbifold $T^{4} / \mathbb{Z}_{2}$ in terms of type IIA string theory. Note that the quotient is a singular $K 3$ manifold. On a smooth $K 3$ manifold there can be no non-trivial RR 1-form fluxes. The fundamental group is trivial so that there can be no non-trivial flat $U(1)$ bundles. So the 1 -form flux is necessarily located at the singularities. This we want to make more precise.

In the case of $T^{4} / \mathbb{Z}_{2}$, the $K 3$ manifold has $16 A_{1}$ singularities that look locally like $\mathbb{C}^{2} / \mathbb{Z}_{2}$. If we cut out a small neighbourhood of the singularities, we obtain a manifold $X_{0}$ with a boundary $Y$ that consists of 16 copies of $S^{3} / \mathbb{Z}_{2} \cong \mathbb{R P}^{3}$. Since $\pi_{1}\left(\mathbb{R P}^{3}\right)=\mathbb{Z}_{2}$ there is a non-contractible curve $\gamma_{p}$ around each fixed point $p$. Since we are given a (smooth) line bundle with a flat connection $A$ over $X_{0}$, we can compute the holonomy of the connection around that curve for every fixed point $p$

$$
\exp i \oint_{\gamma_{p}} A=\exp i \pi \varphi(A ; p), \quad \varphi(A ; p) \in \mathbb{Z}_{2}=\{0,1\} .
$$

The two possibilities $\varphi(A ; p)=0,1$ correspond to the two possible equivariant flat line bundles on $\mathbb{C}^{2}$ with either the trivial or the non-trivial group action.

A clear interpretation of a Type IIA string theory compactification on the orbifold $\mathbb{C}^{2} / \mathbb{Z}_{2}$ with a discrete flux for the RR 1-form gauge field $A$ is given in [15]. This flux can be measured by performing an Aharanov-Bohm scattering process by sending a D0-brane around the loop $\gamma$. Because of this discrete gauge field, the singular orbifold cannot be deformed to a smooth ALE hyperkähler manifold, as in the case without discrete RR flux. Such a deformation would kill the fundamental group and the corresponding nontrivial flat connection $A$. Therefore the singularity is "frozen." String loop corrections make this apparent singular configuration non-singular. In M theory, this compactification is represented by the five-dimensional orbifold

$$
\left(\mathbb{C}^{2} \times S^{1}\right) / \mathbb{Z}_{2}
$$


where the $\mathbb{Z}_{2}$ acts on the circle by $x^{11} \rightarrow x^{11}+\pi$. This action is fixed point free so that we are dealing with a smooth $\mathrm{M}$ theory compactification. In weak coupling, when the radius of the $S^{1}$ shrinks to zero, the string theory becomes free everywhere outside the core of the singularity, but an interacting string theory remains at the singularity, which can be viewed as a dual represention of a Neveu-Schwarz five-brane.

Since there are 16 fixed points, this seems to give a priori $2^{16}$ possibilities for putting discrete flux at the singularity. But in this global situation not all of these possibilities are realized. A constraint comes about because the loops $\gamma_{p}$ are not all independent in the homology group $H_{1}\left(X_{0}, \mathbb{Z}\right)$, where we recall that $X_{0}$ is the orbifold with the singularities cut out. There are relations among the 16 generators. This means that not any arbitrary combination of the 16 fluxes leads to a flat bundle that can be extended over the interior of the $K 3$ manifold.

In fact, it is a classical result in the theory of Kummer surfaces that the first homology group of $X_{0}$ is given by

$$
H_{1}\left(X_{0}, \mathbb{Z}\right) \cong\left(\mathbb{Z}_{2}\right)^{5}
$$

We will explain this result in more detail in the next section where we discuss flat line bundles on singular $K 3$ manifolds in general. For the moment, we just observe that we recover the group $\left(\mathbb{Z}_{2}\right)^{5}$ of the equivariant computation (87).

We now want to connect the local and the global computation. How do we determine the holonomy of a given equivariant bundle on $T^{4}$ around a particular fixed point $p$ ? This is a straightforward computation, see also $[73,74]$.

Note that from an abstract point of view, we are doing the following: consider a neighbourhood $U_{p}$ of the point $p$ on the cover $T^{4}$. This we can identify as a ball in $\mathbb{C}^{2}$ equiped with the non-trivial $\mathbb{Z}_{2}$ action. The inclusion 
$i_{p}: U_{p} \rightarrow T^{4}$ is an equivariant map. It therefore gives rise to a map

$$
i_{p}^{*}: H_{\mathbb{Z}_{2}}^{1}\left(T^{4}, U(1)\right) \rightarrow H_{\mathbb{Z}_{2}}^{1}\left(U_{p}, U(1)\right) \cong \mathbb{Z}_{2}
$$

This map describes the restriction of the bundle on $T^{4}$ to the bundle in the neighbourhood of the fixed point. Locally, there is only the generator $\xi$. So under restriction the equivariant class $A$ will become some multiple of $\xi$ :

$$
i_{p}^{*}(A)=\varphi(A ; p) \xi
$$

and this defines the holonomy $\varphi(A ; p)$ around $p$.

Since the fixed points are the points of order two on $T^{4}$, we can label them by $p=\left(p^{1}, p^{2}, p^{3}, p^{4}\right) \in\left(\mathbb{Z}_{2}\right)^{4}$, so that the fixed point $p$ has coordinates $x^{i}=\pi p^{i}$. We now claim that the holonomy of the bundle $A=a_{i} \theta^{i}+a_{0} \xi$ is given by the linear affine function

$$
\varphi(A ; p)=a_{i} p^{i}+a_{0}
$$

From our abstract description this follows rather directly, because we defined the generator $\theta^{i}$ such that it restricts to zero at $p^{i}=0$ and equals $\theta^{i}=\xi$ at $p^{i}=1$. So we can write the restriction simply as

$$
i_{p}^{*}\left(\theta^{i}\right)=p^{i} \xi
$$

We display this function for the five generators, where we listed the 16 fixed points $p$ lexicographically.

\begin{tabular}{|l|llllllllllllllll|}
\hline$\theta^{1}$ & 1 & 1 & 1 & 1 & 1 & 1 & 1 & 1 & 0 & 0 & 0 & 0 & 0 & 0 & 0 & 0 \\
$\theta^{2}$ & 1 & 1 & 1 & 1 & 0 & 0 & 0 & 0 & 1 & 1 & 1 & 1 & 0 & 0 & 0 & 0 \\
$\theta^{3}$ & 1 & 1 & 0 & 0 & 1 & 1 & 0 & 0 & 1 & 1 & 0 & 0 & 1 & 1 & 0 & 0 \\
$\theta^{4}$ & 1 & 0 & 1 & 0 & 1 & 0 & 1 & 0 & 1 & 0 & 1 & 0 & 1 & 0 & 1 & 0 \\
$\xi$ & 1 & 1 & 1 & 1 & 1 & 1 & 1 & 1 & 1 & 1 & 1 & 1 & 1 & 1 & 1 & 1 \\
\hline
\end{tabular}


Apart from the bundles $A=0$ and $A=\xi$ that have fluxes at none or all of the 16 fixed points, every other bundle has fluxes at 8 of the 16 fixed points. This is in line with the description of the bundle as $\left(T^{4} / \dot{\mathbb{Z}}_{2} \times S^{1}\right) / \mathbb{Z}_{2}$.

We can of course also do a straightforward computation of the holonomy by integrating the gauge field over the closed path $\gamma_{p}$. In terms of the cover $T^{4}$, this loop can be represented as a path that starts at the point $x_{0}=\pi p-\epsilon$ and ends at $x_{1}=\pi p+\epsilon$ with $\epsilon$ a small 4 -vector. This is an open path on $T^{4}$, so to find the holonomy along this path, we have to relate the bundle at the two end points.

The original connection on $T^{4}$ we picked to be the constant one-form $A=\frac{1}{2} a_{i} d x^{i}$. We can gauge this connection away at the cost of introducing twisted boundary conditions. One describes these boundary conditions by accompanying the identification $x^{i} \rightarrow x^{i}+2 \pi n^{i}$, with $n^{i} \in \mathbb{Z}$, by a gauge transformation $\exp i \pi a_{i} n^{i}$. Under the quotient map $x^{i} \rightarrow-x^{i}$, we can have a further gauge transformation $\exp i \pi a_{0}$. With these boundary conditions, we can simply compute the holonomy around the path $\gamma_{p}$. Since the local connection is trivial, the holonomy is complete expressed in the gauge transformation that accompanies the identification of the two end points. Since

$$
x_{1}=-x_{0}+2 \pi p
$$

the gauge transformation gives the phase

$$
\exp i \pi\left(a_{i} p^{i}+a_{0}\right)
$$

which gives our formula (106).

\subsubsection{Singular $K 3$ manifolds with one-form flux}

We will now turn to the case of a general $K 3$ surface. As we explained, smooth $K 3$ 's cannot support 1 -form flux, so the $K 3$ surface is necessarily singular. Locally these singularities will be of type $\mathbb{C}^{2} / G$ with $G$ a finite 
subgroup of $S U(2)$ as given by the $A D E$-classification. This implies that we can consider $X$ to be a local orbifold, i.e., a manifold that can be covered by coordinate patches that are orbifolds. In such a case, we still have welldefined equivariant cohomology groups. However, in the case of 1-form flux, there is no need to use these equivariant classes; instead, we simply excise the singularities and work on the smooth remainder.

This result is formulated as follows using the language of algebraic topology. Let $X_{*}$ be the singuar $K 3, U_{*}$ a neighbourhood of the singularities, and

$$
X_{0}=X_{*}-U_{*}
$$

the smooth manifold obtained by cutting out the singularities. The boundary $\partial X_{0}=Y$ will consist of a union of smooth three-manifolds of the type $S^{3} / G$. We clearly want to compute the group

$$
\mathcal{Z}=H_{1}\left(X_{0}, \mathbb{Z}\right)
$$

Now let $X$ be the resolved $K 3$ surface, and let $U$ be the neighbourhood of the resolved singularities. That is we replace every component of $U_{*}$ by its corresponding smooth ALE-space. We have $X-U=X_{0}$ and $\partial U=Y$. It is a standard result that

$$
H_{1}\left(X_{0}, \mathbb{Z}\right) \cong H^{3}\left(X_{0}, Y, \mathbb{Z}\right) \cong H^{3}(X, U, \mathbb{Z}) .
$$

Here we use the relative cohomology groups $H^{k}(X, U)$. These describe pairs $(a, b) \in C^{k}(X) \times C^{k-1}(U)$ of a $k$-cochain $a$ on $X$ and a $(k-1)$-cochain $b$ on $U$ that satisfy $d a=0, i^{*} a=d b$, modulo the equivalence $(a, b) \sim$ $\left(a+d u, b+i^{*} u-d v\right)$ with $(u, v) \in C^{k-1}(X) \times C^{k-2}(U)$. So the elements of $H^{k}(X, U)$ represent, roughly, cocycles on $X$ that are trivial when restricted to $U$. Lefschetz duality gives

$$
H^{k}(X, U) \cong H_{4-k}(X-U), \quad H^{k}(X-U) \cong H_{4-k}(X, U) .
$$

Relative cohomology groups fit in a long exact sequence

$$
\rightarrow H^{k}(X, U) \rightarrow H^{k}(X) \rightarrow H^{k}(U) \rightarrow H^{k+1}(X, U) \rightarrow
$$


In our case, using the cohomology of $X=K 3$ and $U$, this gives the short exact sequence

$$
0 \rightarrow H^{2}(X, U, \mathbb{Z}) \rightarrow H^{2}(X, \mathbb{Z}) \rightarrow H^{2}(U, \mathbb{Z}) \rightarrow H^{3}(X, U, \mathbb{Z}) \rightarrow 0 .
$$

Here $H^{2}(X, \mathbb{Z}) \cong \Gamma_{3,19}$ is the familiar second cohomology of $K 3$. The group $H^{2}(U, \mathbb{Z})$ is the dual of group of vanishing cycles $Q=H_{2}(U, \mathbb{Z})$. The lattice $Q$ is a sublattice of $H_{2}(X, \mathbb{Z}) \cong \Gamma_{3,19}$. It consist of the 2-cycles that are contracted to a point in the singularity. There is a second sublattice of $H_{2}(X, \mathbb{Z})$, the lattice $P=H_{2}\left(X_{0}, \mathbb{Z}\right)$ of 2-cycles in the smooth part $X_{0}$. Duality gives us $P \cong H^{2}(X, U, \mathbb{Z}) \cong H^{2}\left(X_{0}, Y, \mathbb{Z}\right)$. So $P$ represents the bundles that become trivial on the boundary $Y$. Using these results the exact sequence gives

$$
0 \rightarrow P \rightarrow \Gamma_{3,19} \rightarrow Q^{*} \rightarrow \mathcal{Z} \rightarrow 0 .
$$

This equation has the following interpretation: the singularity gives rise to a sublattice $Q \subset \Gamma_{3,19}$ of vanishing cycles. This gives a dual map $\Gamma_{3,19} \rightarrow Q^{*}$. This map is not necessarily onto. The quotient of $Q^{*}$ by the image of $\Gamma_{3,19}$ gives us the group of 1-form fluxes $\mathcal{Z}$. Equivalently, the lattice $Q \subset \Gamma_{3,19}$ is not necessarily primitively embedded, and this is measured by $\mathcal{Z}$.

There are some other definitions of $\mathcal{Z}$ that are directly equivalent to this by duality. Since we also have

$$
\mathcal{Z}=\operatorname{Tor} H^{2}\left(X_{0}, \mathbb{Z}\right) \cong \operatorname{Tor} H_{2}(X, U, \mathbb{Z}),
$$

we find for example

$$
H^{2}\left(X_{0}, \mathbb{Z}\right)=H_{2}(X, U, \mathbb{Z}) \cong P^{*} \oplus \mathcal{Z} .
$$

Note that the lattices $Q$ and $Q^{*}$ have an interpretation in terms of line bundles over the resolved singularity $U$. The exact sequence

$$
0 \rightarrow H^{2}(U, Y, \mathbb{Z}) \rightarrow H^{2}(U, \mathbb{Z}) \rightarrow H^{2}(Y, \mathbb{Z}) \rightarrow 0
$$

becomes

$$
0 \rightarrow Q \rightarrow Q^{*} \rightarrow \operatorname{disc}(Q) \rightarrow 0
$$


where the discriminant of the lattice $Q$ also equals the first homology of the three-manifold $Y$, which is a disjoint sum of quotients $S^{3} / G$. To be precise, $\operatorname{disc}\left(A_{n}\right)=\mathbb{Z}_{n+1}, \operatorname{disc}\left(D_{n}\right)=\mathbb{Z}_{2} \times \mathbb{Z}_{2}$ or $\mathbb{Z}_{4}$ for $n$ even or odd, respectively, and $\operatorname{disc}\left(E_{n}\right)=\mathbb{Z}_{3}, \mathbb{Z}_{2},\{1\}$ for $n=6,7$ and 8 , respectively. Here $Q^{*}=H^{2}(U, \mathbb{Z})$ are the line bundles on $U, Q=H^{2}(U, Y, \mathbb{Z})$ are the line bundles that become trivial when restricted to $Y$, and $\operatorname{disc}(Q)=H^{2}(Y, \mathbb{Z}) \cong$ $H_{1}(Y, \mathbb{Z})$ represent the (necessarily torsion) line bundles on $Y$.

\subsection{Fluxes and K-theory}

We now turn to the K-theory description of fluxes for the RR 1-form and 3 -form fields in type IIA string theory.

\subsubsection{K-theory description of RR fluxes}

There is now considerable evidence that K-theory should play a role in classifying RR charges in type II string theory $[75,76]$, at least for zero string coupling. There are also arguments suggesting that the RR fields themselves, and in particular their flux quantization, should be formulated in terms of $\mathrm{K}$ theory $[77,78]$. To be more precise, there is a local formulation of K-theory called differential K-theory that provides a precise characterization of the $\mathrm{RR}$ fields in terms of bundles with connections [79]. According to this point of view the flat RR fluxes on a compactification $X$ are given by the groups $K^{i}(X, U(1))$ where $i=1$ for the IIA theory and $i=0$ for the IIB ( $i$ only matters modulo 2 by Bott periodicity). These groups have been studied in detail in [80].

The most important property of the groups $K^{i}(X, U(1))$ is that they fit in the exact sequence

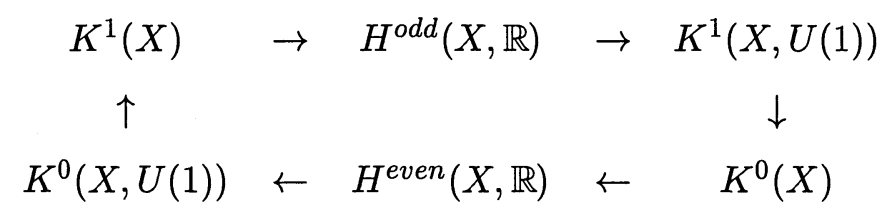


Here the maps from K-theory to cohomology are the usual Chern character maps, that are isomorphisms over the reals.

For a finite-dimensional smooth manifold $X$, we therefore find a description of the RR phases as

$$
0 \rightarrow \frac{H^{\text {odd }}(X, \mathbb{R})}{K^{1}(X) / \text { Tor }} \rightarrow K^{1}(X, U(1)) \rightarrow \text { Tor } K^{0}(X) \rightarrow 0 .
$$

So the component group is given by the torsion classes in $K^{0}(X)$, and the trivial component consists of the torus $H^{\text {odd }}(X, \mathbb{R}) /\left(K^{1}(X) /\right.$ Tor $)$.

$\mathrm{K}$-theory is not graded, so one cannot distinguish in a unique way the contributions of the RR fields of fixed degree. Instead there is a filtration

$$
K^{0}(X) \supseteq K_{1}^{0} \supseteq K_{2}^{0} \supseteq \cdots
$$

where the group $K_{k}^{0}(X)$ is defined in terms of vector bundles that are trivial on the $(k-1)$-skeleton of $X$. This gives a description of $\mathrm{RR}$ fluxes where the field strength is a form of degree $k$ or higher. So, within the $\mathrm{K}$-theory formalism fluxes always have ambiguities up to higher degree terms.

In this paper we want to restrict to fluxes of the 1-forms and 3-forms in the IIA theory as measured by the world-volumes of D0-branes and D2branes. These are given by the quotient

$$
K^{1}(X, U(1)) /\left(K^{1}(X, U(1))_{5} .\right.
$$

We will not give the general interpretation of the elements of the group $K^{1}(X, U(1))$, but restrict our discussion here to the case that $H^{\text {odd }}(X, \mathbb{R})$ vanishes, so that only torsion classes appear. In that case the group of fluxes is simply

$$
K^{1}(X, U(1)) \cong \operatorname{Tor} K^{0}(X) .
$$

The corresponding RR flux can be represented by a pair $\left(E_{0}, E_{1}\right)$ of flat vector bundles with $\operatorname{rk}\left(E_{0}\right)=\operatorname{rk}\left(E_{1}\right)$, i.e., a virtual flat bundle $E_{0}-E_{1}$ of rank zero. 
A torsion RR flux will give an additional phase factor to a D-brane in the string theory path-integral. If we represent a brane by a K-homology class, that is a map an odd spin-manifold $M$ (possibly equipped with an additional Chan-Paton vector bundle $F$ ) into the space-time $X$, the holonomy of the RR-fields over this brane can be represented by the $\eta$-invariant of virtual bundle $E_{0}-E_{1}$ restricted to $M$.

More precisely, consider the Dirac operator $D$ on $M$ coupled to the bundles $E_{0}$ and $E_{1}$. Such a Dirac operator is self-adjoint and has a $\eta$-invariant, which is the regularized sum of the signs of the eigenvalues $\lambda_{i}$ of $D$ [81]

$$
\eta=\eta(0), \quad \eta(s)=\sum_{\lambda_{i} \neq 0} \operatorname{sgn}\left(\lambda_{i}\right)\left|\lambda_{i}\right|^{-s} .
$$

Closely related is the so-called reduced $\eta$-invariant, defined by

$$
\bar{\eta}=(\eta+\operatorname{dim} \operatorname{ker} D) / 2 \quad(\bmod \mathbb{Z}) .
$$

This invariant does not jump under variations of the parameters. It appears in the index theorem on manifold with boundaries. If we can write $M$ as the boundary of a manifold $B$, and extend the bundle $E_{0}$ to a bundle $E$ over $Z$, then with the appropriate APS boundary conditions, the twisted Dirac operator $D_{E}$ on $B$ has index

$$
\operatorname{index} D_{E}=\int_{B} \operatorname{ch}(E) \widehat{A}(B)-\bar{\eta}_{E_{0}}
$$

The phase (modulo $\mathbb{Z}$ ) associated to $M$ is now

$$
\varphi(M)=\bar{\eta}_{E_{0}}-\bar{\eta}_{E_{1}}
$$

In view of the APS index theorem, this can be written as

$$
\varphi(M)=\int_{M \times I} \operatorname{ch}(E) \widehat{A}(M)
$$

where we have picked a bundle $E$ with a (no longer necessarily flat) connection on the manifold $M \times I$ with $I=[0,1]$ that reduces to $E_{0}$ and $E_{1}$ on the two boundaries. 


\subsubsection{K-theory on orbifolds}

For the case of an orbifold $X / G$, it seems reasonable to assume that equivariant K-theory is the appropriate description. For the case of the RR charges the relevance of equivariant $\mathrm{K}$-theory has been demonstrated in great detail [75,82-84]. It leads to an elegant description of fractional branes pinned on the orbifold singularities. The boundary state corresponding to these fractional branes has components in the twisted sectors of the closed string.

Equivariant K-theory $K_{G}(X)$ classifies equivalence classes of equivariant bundles on $X$, that is bundles with an action of $G$. Just as in equivariant cohomology, we can make a model for $K_{G}(X)$ in terms of the ordinary Ktheory on the homotopy quotient $X_{G}$. However, in the equivariant case there is a remarkable difference in the prediction of fluxes coming from a $\mathrm{K}$-theory or a cohomology formulation.

As an example we can study the basic orbifold $\mathbb{C}^{2} / \mathbb{Z}_{2}$ again. In this case we have

$$
K_{\mathbb{Z}_{2}}^{0}\left(\mathbb{C}^{2}\right) \cong K_{\mathbb{Z}_{2}}^{0}(p t) \cong K^{0}\left(\mathbb{R} \mathbb{P}^{\infty}\right)=\mathbb{Z} \oplus \mathbb{Z}
$$

This generalizes as follows. For an arbitrary finite group $G$ the $\operatorname{ring} K_{G}^{0}(p t)$ is given by the (completion of the) representation $\operatorname{ring} R(G)$ of $G$. One has $R(G)=\mathbb{Z}^{r}$ with $r$ the number of irreducible representations (or equivalently the number of conjugacy classes). So as an example for $\mathbb{Z}_{2}$, the representation ring is generated by the trivial and the non-trivial representation.

The equivariant K-group should be contrasted by the equivariant cohomology that is given by

$$
H_{\mathbb{Z}_{2}}^{\text {even }}\left(\mathbb{C}^{2}, \mathbb{Z}\right)=H^{\text {even }}\left(\mathbb{R P}^{\infty}, \mathbb{Z}\right)=\mathbb{Z} \oplus \mathbb{Z}_{2} \oplus \mathbb{Z}_{2} \oplus \cdots
$$

This difference is a consequence of a familiar effect: if we consider a finitedimensional approximation by $\mathbb{R}^{2 N+1}$ then the even cohomology and $\mathrm{K}$ theory have the same associated graded, but differ in the extensions

$$
K^{0}\left(\mathbb{R P}^{2 N+1}\right)=\mathbb{Z} \oplus \mathbb{Z}_{2^{N}}, \quad H^{\text {even }}\left(\mathbb{R}^{2 N+1}, \mathbb{Z}\right)=\mathbb{Z} \oplus\left(\mathbb{Z}_{2}\right)^{N} .
$$


We see how in the large $N$ limit the $\mathrm{K}$-group becomes free because $\mathbb{Z}_{2^{N}}$ tends to $\mathbb{Z}$. This effect has been recently described in terms of fractional branes on the orbifold in [84].

According to the equivariant version of the sequence (121), the IIA RR fluxes for the orbifold $\mathbb{C}^{2} / G$ are given by the kernel of the map

$$
K_{G}^{0}(p t) \rightarrow H^{e v e n}(G, \mathbb{R}) \cong \mathbb{R}
$$

This is the so-called reduced K-theory $\widetilde{K}_{G}^{0}(p t)$ that is represented by virtual bundles of dimension zero. So we have the prediction that the group of fluxes is given by

$$
K_{G}^{1}\left(\mathbb{C}^{2}, U(1)\right) \cong \widetilde{K}_{G}^{0}(p t) \cong \widetilde{R}(G)
$$

Here $\widetilde{R}(G)$ is the reduced representation ring that consists of virtual representations of dimension zero, i.e., pairs of representations

$$
\rho_{0}, \rho_{1}: G \rightarrow U(N)
$$

with $\operatorname{dim} \rho_{0}=\operatorname{dim} \rho_{1}$. Given such a representation, we can construct the corresponding equivariant flat bundles $E_{0}, E_{1}$ on $\mathbb{C}^{2}$, by taking trivial bundles $\mathbb{C}^{N}$ and implementing the action of $G$ through the representations $\rho_{0}$, $\rho_{1}$.

Now this description raises some questions. It seems to predict an infinite set of fluxes at a simple orbifold. If $G$ is of $A D E$-type, then the lattice $\widetilde{K}_{G}^{0}(p t)$ can be identified with the weight lattice $Q^{*}$ of the corresponding simple Lie group. However, as we remarked before, in a K-theory description one cannot distinguish the dimension of the corresponding flux. Since the equivariant fluxes are defined in terms of an infinite-dimensional classifying space, this would imply that the fluxes are of arbitrary high dimension and should be measured by branes of arbitrary high dimension. This is clearly not a physical description. In our case one would like to restrict to 1-form and 3-form fluxes that can be measured by the world-volumes of zero-branes and two-branes. Therefore one would argue that the physical fluxes are 
restricted to the quotient

$$
\widetilde{K}_{G}^{0}(p t) /\left(\widetilde{K}_{G}^{0}(p t)\right)_{5}
$$

where we have taken the quotient by the subgroup that describes bundles that vanish on the 4-skeleton of $B G$.

\subsubsection{One-form fluxes in K-theory}

How do we measure such a flux in the orbifold $\mathbb{C}^{2} / G$ ? As we explained above, a RR flux flux will give an extra phase to an Euclidean D-brane instanton. Let us also restrict to branes with smooth world-volumes that do not pass through the orbifold singularity. So we consider the space $\left(\mathbb{C}^{2}-\{0\}\right) / G$. This can be retracted to the smooth 3-dimensional space $S^{3} / G$.

Let us first consider the RR 1-form field. Fluxes of this field are described by the quotient

$$
\widetilde{K}_{G}^{0}(p t) /\left(\widetilde{K}_{G}^{0}(p t)\right)_{3} .
$$

Given a closed one-manifold $\gamma$ in $S^{3} / G$ the holonomy of the 1-form field is described as follows. The K-theory class is given by the pair of representations $\left(\rho_{0}, \rho_{1}\right)$ and the corresponding flat equivariant bundles $\left(E_{0}, E_{1}\right)$. To such a virtual vector bundle we can associate a flat equivariant line bundle $L$, the determinant bundle

$$
L=\operatorname{det} E_{0} \otimes\left(\operatorname{det} E_{1}\right)^{*}
$$

Now the phase of the RR 1-form field is simply the holonomy of the flat connection $A$ of the line bundle $L$

$$
\exp i \oint_{\gamma} A \text {. }
$$

The first Chern class of this line bundle is represented by an equivariant cohomology class

$$
c_{1}(L)=c_{1}\left(\rho_{0}\right)-c_{1}\left(\rho_{1}\right) \in H^{2}(G, \mathbb{Z})
$$


Here we define the Chern classes of a representation $\rho: G \rightarrow U$ through the associated map of classifying spaces

$$
\rho^{*}: H^{*}(B U) \rightarrow H^{*}(G, \mathbb{Z}) .
$$

The cohomology of the classifying space $B U$ is generated by the Chern classes $c_{1}, c_{2}, \ldots$ and the images $\rho^{*} c_{i}$ we denote by $c_{i}(\rho)$.

Since any element of $H^{2}(G, \mathbb{Z})$ represent an equivariant line bundle, we see that the equivariant $\mathrm{K}$-theory description coincides with the description in terms of equivariant cohomology classes given in section 4.4. The fluxes are given by

$$
H^{2}(G, \mathbb{Z}) \cong H^{1}(G, U(1)) .
$$

\subsubsection{Three-form fluxes in K-theory}

According to the formalism explained in the preceeding discussion, a 3-form flux will associate a phase to a Euclidean D2-brane world-volume $M$ given by the eta-invariant of the virtual bundle $E_{0}-E_{1}$ restricted to $M$

$$
\varphi(M)=\bar{\eta}_{E_{0}}-\bar{\eta}_{E_{1}} .
$$

In our case there is an obvious choice for $M$, namely the manifold $S^{3} / G$ that surrounds the singularity. (Note that any oriented three-manifold is spin, although there could be in principle more than one inequivalent spin structure.) We can express this phase directly in terms of the Chern-Simons invariant, since

$$
\varphi(M)=\int_{M \times I} \operatorname{ch}(E)=C S\left(E_{0}\right)-C S\left(E_{1}\right) .
$$

Here we have defined the Chern-Simons invariant for a $U(N)$ flat gauge field as

$$
C S(E)=\int_{B} c h_{2}(E)
$$


where $B$ is a four-manifold with $\partial B=M$ over which we have extended the bundle $E$ and $c h_{2}$ is the second Chern character

$$
c h_{2}=\frac{1}{2} c_{1}^{2}-c_{2}=\frac{1}{8 \pi^{2}} \operatorname{Tr} F \wedge F .
$$

For the $U(1)$ part, this is half the usual Chern-Simons term. As explained in [85], this is perfectly fine. Because the relevant cobordism groups vanish, for every three-manifold $M$ one can find a four-manifold $B$ and an extension of the $U(N)$ bundle such that $B$ is spin. The fact that the intersection form on $B$ is now even allows one to define the $C S$ invariant using $\frac{1}{2} c_{1}^{2}$.

Given a representation $\rho$ of $G$, we can compute the Chern-Simons invariant in terms of group cohomology as follows. We have the Chern classes

$$
c_{1}(\rho) \in H^{2}(G, \mathbb{Z}), \quad c_{2}(\rho) \in H^{4}(G, \mathbb{Z}) .
$$

Now it is not difficult to see that for a group $G$ of $A D E$-type, we have

$$
H^{4}(G, \mathbb{Z}) \cong \mathbb{Z}_{|G|}
$$

In fact a spectral sequence argument [86] gives $H^{4}(G, \mathbb{Z})$ as the cokernel of the composition

$$
H^{3}(S U(2) / G, \mathbb{Z}) \rightarrow H^{3}(S U(2), \mathbb{Z}) \rightarrow H^{4}(B S U(2), \mathbb{Z}) .
$$

The first map is clearly of order $|G|$ whereas the second (transgression) is an isomorphism. So the generator of $H^{4}(G, \mathbb{Z})$ is the class $c_{2}\left(\rho_{2}\right)$ where $\rho_{2}$ is the defining 2-dimensional representation of $G$.

The $C S$ invariant for the manifold $S^{3} / G$ is defined by the map

$$
C S: H^{3}(G, U(1)) \rightarrow H^{3}\left(S^{3} / G, U(1)\right) \cong U(1)
$$

where we identify $S^{3} \cong S U(2)$. So for the representation $\rho_{2}$, this is simply given by

$$
C S\left(\rho_{2}\right)=\frac{1}{|G|}(\bmod \mathbb{Z})
$$


For a general representation $\rho$, let $c_{1}$ be its first Chern class considered as an element of $H^{2}\left(S^{3} / G, \mathbb{Z}\right) \cong H^{2}(G, \mathbb{Z})$, and let $\alpha$ be the representative in $H^{1}\left(S^{3} / G, U(1)\right)$. Similarly, let $\beta$ be the representative of $c_{2}$ in $H^{3}\left(S^{3} / G, U(1)\right)$. Then the Chern-Simons invariant of $\rho$ is defined by

$$
C S(\rho)=\frac{1}{2} \alpha \cup c_{1}-\beta .
$$

The phase associated to a D2-brane wrapped on $S^{3} / G$ in a background described by the virtual flat bundle $\rho_{0}-\rho_{1}$ is given by

$$
\varphi\left(S^{3} / G\right)=C S\left(\rho_{0}\right)-C S\left(\rho_{1}\right) .
$$

Note that the first term in the Chern-Simons term (coming from $\frac{1}{2} c_{1}^{2}$ ) has a familiar description. There is an isomorphism

$$
H^{2}(G, \mathbb{Z}) \cong Q^{*} / Q
$$

where $Q$ is the root lattice of type $G$. Since $Q$ is an even lattice, there is a quadratic form (essentially the discriminant form of the lattice $Q$-see appendix A),

$$
x \in Q^{*} / Q \mapsto q(x)=\frac{1}{2} x \cdot x \in \mathbb{Q} / \mathbb{Z} \subset U(1) .
$$

This is the $C S$ invariant of the line bundle $x$.

If the 1-form flux is zero, we can define the group of 3 -form fluxes as the quotient

$$
\left(\widetilde{K}_{G}^{0}\right)_{3} /\left(\widetilde{K}_{G}^{0}\right)_{5}
$$

In this case $c_{1}=0$ so the flux is measured by $c_{2} \in H^{3}(G, U(1))$. Since this group is generated by the 2-dimensional representation, we see that this group of pure 3 -form fluxes is simply given by

$$
H^{4}(G, \mathbb{Z})=H^{3}\left(G, U(1)=Z_{|G|} .\right.
$$

Indeed, in this case it is not difficult to compute the groups of fluxes directly in K-theory. For a finite subgroup $G \subset S U(2)$, the non-vanishing 
cohomology occurs in even degree, where for $k \geq 1$

$$
H^{4 k-2}(G, \mathbb{Z})=G_{a b}=G /[G, G], \quad H^{4 k}(G, \mathbb{Z})=\mathbb{Z}_{|G|} \cdot
$$

This implies immediately that the Atiyah-Hirzebruch spectral sequence degenerates. If

$$
K_{p}=\left(\widetilde{K}_{G}^{0}(p t)\right)_{p}
$$

then we have the successive quotients

$$
K_{p} / K_{p+1}=H^{p}(G, \mathbb{Z}), \quad p>0 .
$$

This immediately gives that the group of 1-form fluxes modulo 3 -form fluxes is

$$
K_{0} / K_{3}=H^{2}(G, \mathbb{Z})
$$

as we have seen explicitly. The group of 3 -form fluxes with vanishing 1-form flux is also easily computed to be

$$
K_{3} / K_{5} \cong H^{4}(G, \mathbb{Z})
$$

confirming our computation via the $C S$ invariants. Finally the full group of 1-form and 3-form fluxes

$$
M(G)=K_{0} / K_{5}
$$

is given by an extension

$$
0 \rightarrow H^{4}(G, \mathbb{Z}) \cong \mathbb{Z}_{|G|} \rightarrow M(G) \rightarrow H^{2}(G, \mathbb{Z}) \rightarrow 0 .
$$

The corresponding cocycle of this extension is given by the map

$$
b: H^{2}(G: \mathbb{Z}) \times H^{2}(G, \mathbb{Z}) \rightarrow H^{4}(G, \mathbb{Z}), \quad b\left(x, x^{\prime}\right)=x \cup x^{\prime} .
$$

This cocycle is definitely exact as an extension by $U(1)$ since

$$
b\left(x, x^{\prime}\right)=q\left(x+x^{\prime}\right)-q(x)-q\left(x^{\prime}\right), \quad q(x)=\frac{1}{2} x \cdot x
$$


where we use the identification $H^{2}(G, \mathbb{Z}) \cong Q^{*} / Q$. So the question is simply whether $q(x)$ is of the form $n /|G|$. Since $2 q(x)=x \cdot x$ is always in $H^{4}(G, \mathbb{Z})$, the only question is whether $x \cdot x$ is divisible by two in $H^{4}(G, \mathbb{Z})$.

This extension question changes the additive structure on the group of fluxes. Physically this effect can be described as follows. In the presence of a RR 1-form background $x \in H^{2}(G, \mathbb{Z})$, there is an induced 3-form flux given by the $C S$ invariant

$$
C S(x)=\int_{B} \frac{1}{2} c_{1}^{2}=q(x) .
$$

Now suppose $x$ has order $n$. That is to say, $x$ represents a line bundle $L$ and $n x$ represents the trivial line bundle $L^{\otimes n}$. The 1-form flux of $n x$ as measured by D0-branes vanishes, but the 3 -form flux will be given by $n q(x)$ and this is not necessarily $0 \bmod 1$. As we argued above one can have $n q(x)=\frac{1}{2} \bmod$ 1.

In terms of $\mathrm{K}$-theory, such an element $x$ corresponds to a virtual bundle $L-1$. But this element has infinite order using the group structure in $\mathrm{K}$ theory which is addition of bundles. If we take it $n$ times, we get the class $n L-n$ which is a virtual vector bundle. Of course the determinant line is trivial, but the bundle has higher order secondary invariants, in particular the $C S$ invariant measured by a $\mathrm{D} 2$-brane.

The simplest example is $G=\mathbb{Z}_{2}$. In that case we have

$$
K_{0}=\widetilde{K}_{\mathbb{Z}_{2}}^{0}(p t)=\mathbb{Z}
$$

generated by the class $x=\rho-1$ where $\rho$ is the non-trivial representation of $\mathbb{Z}_{2}$ (or the tautological line bundle over $\mathbb{R} \mathbb{P}^{\infty}$ ). We can now compute the phases for a flux $n x, n \in \mathbb{Z}$. As we explained we are only interested in the 1-form and 3-form fluxes. These are labeled by

$$
M\left(\mathbb{Z}_{2}\right)=K_{0} / K_{5}=\lim _{N \rightarrow \infty} \mathbb{Z}_{2^{N}} / \mathbb{Z}_{2^{N-2}} \cong \mathbb{Z}_{4}
$$

This should be contrasted with the answer obtained from equivariant coho- 
$\operatorname{mology}^{21}$

$$
H^{2}\left(\mathbb{Z}_{2}, \mathbb{Z}\right) \oplus H^{4}\left(\mathbb{Z}_{2}, \mathbb{Z}\right)=\mathbb{Z}_{2} \oplus \mathbb{Z}_{2}
$$

Since $c_{1}(x)$ is the generator of $H^{2}\left(\mathbb{Z}_{2}, \mathbb{Z}\right) \cong \mathbb{Z}_{2}$, the 1 -form flux is simply $n / 2$ $(\bmod 1)$. So the holonomy of the virtual bundle $n x$ is $(-1)^{n}$.

For the 3-form flux, we have to measure the phase of a membrane wrapped over $S^{3} / \mathbb{Z}_{2}$. This equals the Chern-Simons invariant of the bundle $\rho$. Since $c_{2}=0$ we obtain

$$
C S(x)=C S(\rho)=\int_{B} \frac{1}{2} c_{1}^{2}=\frac{1}{4} .
$$

For the bundle $n x$ we have

$$
C S(n x)=\frac{n}{4} \quad(\bmod \mathbb{Z}) .
$$

We see that in the presence of a RR 1-form flux (that is, if $n$ is odd), there an induced 3-form flux. More precisely, the 3-form flux has a shifted quantization, where the phase factor satisfies

$$
\varphi=\frac{1}{4} \quad\left(\bmod \frac{1}{2}\right)
$$

This computation can be repeated for general $G$. We use the same notation for the root lattice $Q$ and the corresponding finite subgroup $G$ of $S U(2)$. For $A_{n}$ we have an extension

$$
0 \rightarrow \mathbb{Z}_{n+1} \rightarrow M \rightarrow \mathbb{Z}_{n+1} \rightarrow 0
$$

Let $x$ be the generator of $H^{2}\left(A_{n}, \mathbb{Z}\right)=\mathbb{Z}_{n+1}$. One computes $q(x)=n / 2(n+$ 1) so that $(n+1) q(x)=n / 2$. For $n$ odd this is $\frac{1}{2} \bmod 1$, so we find a non-trivial extension

$$
M\left(A_{2 k}\right)=\mathbb{Z}_{2 k+1} \times \mathbb{Z}_{2 k+1}, \quad M\left(A_{2 k-1}\right)=\mathbb{Z}_{4 k} \times \mathbb{Z}_{k} .
$$

\footnotetext{
${ }^{21}$ An explanation about how to compute this equivariant cohomology group appears in appendix D.
} 
For $D_{n}(n>3)$ one finds $H^{2}\left(D_{n}, \mathbb{Z}\right)=\mathbb{Z}_{2} \times \mathbb{Z}_{2}$ for $n$ even, $H^{2}\left(D_{n}, \mathbb{Z}\right)=\mathbb{Z}_{4}$ for $n$ odd, $H^{4}\left(D_{n}, \mathbb{Z}\right)=\mathbb{Z}_{4(n-2)}$ and $q(x)=\frac{n}{8} \bmod 1$ for the generator(s). So, again for $n$ odd we have a non-trivial extension since $4 q(x)=n / 2$

$$
M\left(D_{2 k}\right)=\mathbb{Z}_{2} \times \mathbb{Z}_{2} \times \mathbb{Z}_{8(k-1)}, \quad M\left(D_{2 k-1}\right)=\mathbb{Z}_{8} \times \mathbb{Z}_{2(2 k-3)} .
$$

For $E_{6,7,8}$ the induced fluxes $q(x)$ are respectively $\frac{2}{3}, \frac{3}{4}, 0$ and there are no extensions

$$
M\left(E_{6}\right)=\mathbb{Z}_{3} \times \mathbb{Z}_{24}, \quad M\left(E_{7}\right)=\mathbb{Z}_{2} \times \mathbb{Z}_{48}, \quad M\left(E_{8}\right)=\mathbb{Z}_{120} .
$$

Note that $E_{8}$ does not support 1-form flux.

\subsubsection{An alternate method of computation}

In this section, let us make a brief digression and describe a purely algebraic construction of the quotients

$$
K_{G}^{0}(p t) /\left(K_{G}^{0}(p t)\right)_{2 n+1}
$$

which appeared in our preceeding discussion of fluxes. We shall discuss the case $G=\mathbb{Z}_{m}$. Recall that $K_{2 n}^{0}(X)$ involves bundles that are trivial on the $(2 n)$-skeleton of $X$. For the equivariant case, we need $X=B G$, and a convenient set of spaces that approximate $X$ is given by the set $B G^{(n)}$ that appears in Milnor's construction of $B G$, see, for example [87]. Motivated by the results of [87] and the calculations in appendix D, we propose the following characterization of $K_{\mathbb{Z}_{m}}^{0}(p t) /\left(K_{\mathbb{Z}_{m}}^{0}(p t)\right)_{2 n+1}$. Let $\phi$ be the augmentation map

$$
\phi: \quad R[G] \rightarrow \mathbb{Z}
$$

that sends $\sum n_{i} R_{i}$ to $\sum n_{i} d_{i}$. Here $d_{i}$ is the dimension of the representation $R_{i}$. The kernel of $\phi$ is the augmentation ideal $I$ of $R[G]$. Then

$$
K_{\mathbb{Z}_{m}}^{0}(p t) /\left(K_{\mathbb{Z}_{m}}^{0}(p t)\right)_{2 n-1} \cong R\left[\mathbb{Z}_{m}\right] / I^{n}
$$


For example, for $G=\mathbb{Z}_{2}$ the augmentation ideal is generated by $\rho-1$, where $\rho$ is the non-trivial representation of $\mathbb{Z}_{2}$. Since $(\rho-1)^{n}=2^{n-1}(\rho-1), I^{n}$ is generated by $2^{n-1}(\rho-1)$. From this, we see that

$$
K_{\mathbb{Z}_{2}}^{0}(p t) /\left(K_{\mathbb{Z}_{2}}^{0}(p t)\right)_{2 n-1} \cong \mathbb{Z} \oplus \mathbb{Z}_{2^{n-1}}
$$

in agreement with the result that we found before. A few additional calculations are needed to show that

$$
\begin{aligned}
& K_{\mathbb{Z}_{m}}^{0}(p t) /\left(K_{\mathbb{Z}_{m}}^{0}(p t)\right)_{3}=\mathbb{Z} \oplus \mathbb{Z}_{m} \\
& K_{\mathbb{Z}_{m}}^{0}(p t) /\left(K_{\mathbb{Z}_{m}}^{0}(p t)\right)_{5}=\mathbb{Z} \oplus \mathbb{Z}_{d_{1}} \oplus \mathbb{Z}_{m^{2} / d_{1}} \\
& K_{\mathbb{Z}_{m}}^{0}(p t) /\left(K_{\mathbb{Z}_{m}}^{0}(p t)\right)_{7}=\mathbb{Z} \oplus \mathbb{Z}_{d_{1}} \oplus \mathbb{Z}_{d_{2}} \oplus \mathbb{Z}_{m^{3} / d_{1} d_{2}}
\end{aligned}
$$

where

$$
d_{1}=\operatorname{gcd}\left(m, \frac{m(m-1)}{2}\right), \quad d_{2}=\operatorname{gcd}\left(m, \frac{m(m-1)}{2}, \frac{m(m-1)(m-2)}{6}\right) .
$$

These groups agree with the results of our earlier computations using the reduced eta-invariant.

\section{6 $M$ theory compactifications with three-form flux}

We now turn to $M$ theory compactifications with 3-form flux. In studying these compactifications, we face a difficult issue: namely, we do not yet understand how to correctly treat the 3-form of $\mathrm{M}$ theory. There are various suggestions involving gerbes [88], or suggestive relations with $E_{8}$ gauge bundles $[4,89]$. However, it seems likely that the correct way to treat the 3 -form involves a framework that is currently unknown. In the following section, we describe how the data we have obtained point to the existence of new "frozen" singularities which support 3-form flux. We also conjecture the existence of a new class of dualities between compactifications with different singular geometries and fluxes.

In section 4.6.2, we consider the possibility that equivariant cohomology is the right framework for the 3 -form. As an example, we describe the relevant equivariant cohomology groups for the global orbifold $T^{4} / \mathbb{Z}_{2}$. Section 
4.6.3 extends the discussion of 1-form holonomies which appeared in section 4.4.2 to the case of 3 -form fluxes. This leads to some quite beautiful results which are likely to be useful in other contexts. In section 4.6.4, we compute the equivariant cohomology of the global orbifold $T^{4} / \widehat{\mathcal{D}}_{4}$. We point out that there is a choice of flux with the properties we expect for the M theory compactification dual to the CHL string.

In section 4.6.5, we resolve some puzzles in comparing $M$ theory with type IIA. The resolution suggests a natural generalization of the Freed-Witten anomaly. Section 4.6 .6 contains some thoughts on the the geometry of the $\mathrm{M}$ theory 3-form, and a number of related topics. Finally, we wrap up the discussion with a brief mention of $\mathrm{F}$ theory compactifications with flux.

\subsubsection{Frozen singularities and new dualities}

So far, we have related our asymmetric orbifolds in 6 dimensions to $M$ theory compactifications on $\left(Z \times S^{1}\right) / G$ where $Z=K 3$. We have also described cases with $Z=T^{4}$ without heterotic duals. As we have seen, the smooth geometric $\mathrm{M}$ theory compactifications give type IIA compactifications with torsion RR 1-form flux.

Some of these theories are obtained by compactifying a 7-dimensional theory on a circle. We studied three classes of these 7-dimensional theories: namely, type IIA orientifolds, heterotic asymmetric orbifolds and $\mathrm{F}$ theory compactifications. An example like the CHL string can be realized by all three constructions.

However, it is natural to ask whether there are other strong coupling descriptions which involve $M$ theory compactified on a four-manifold. Since the only bosonic fields of $\mathrm{M}$ theory are the metric and the 3-form field, we can only turn on 3-form fluxes on the four-manifold. Compactifying such a theory on a circle gives a 6-dimensional IIA compactification on the same four-manifold with RR 3-form flux. 
Let us begin by recalling what is known about 7-dimensional $M$ theory compactifications. Apart from the standard compactification of $M$ theory on $\mathrm{K} 3$ without flux, only the strong coupling limit of the CHL string has been discussed. The CHL string can be realized by a 7-dimensional orientifold with $2 \mathrm{O6}^{+}$planes and $6 \mathrm{O6}^{-}$planes. The strong coupling limit of $\mathrm{O6}^{-}$ planes is smooth in $\mathrm{M}$ theory [90-92], while that of an $\mathrm{O6}^{+}$plane appears to be a "frozen" $D_{4}$ singularity in M theory $[16,17]$. The $D_{4}$ singularity can be seen to arise in various ways. In type IIA near an $\mathrm{O6}^{+}$plane, the geometry of a three-surface around the orientifold is $S^{2} / \mathbb{Z}_{2}=\mathbb{R P}^{2}$. In addition, the $\mathrm{O}^{+}$orientifold has $\mathrm{RR}$ charge +4 , which is measured by the field strength of the RR 1-form gauge field. This means that the circle on which we reduce from $\mathrm{M}$ theory to type IIA forms a circle bundle with first Chern class +2 on $\mathbb{R P}^{2}$. The total space of that circle bundle over $\mathbb{R P}^{2}$ is then $S^{3} / D_{4}$. This explains why we expect a $D_{4}$ singularity to appear. Alternatively, before modding out by $\mathbb{Z}_{2}$, we have an object with charge +4 which is therefore described by an $A_{3}$ singularity in $\mathrm{M}$ theory. Modding out by $\mathbb{Z}_{2}$ yields a $D_{4}=A_{3} / \mathbb{Z}_{2}$ singularity.

The same $D_{4}$ singularity can also be found in the geometry seen by a D2-brane probe. On the probe, there is an $N=4 d=3$ Yang-Mills theory with gauge group $O(2)$ and a hypermultiplet transforming in the symmetric representation. The Coulomb branch geometry contains a $D_{4}$ singularity. The existence of this $D_{4}$ singularity is also required to reproduce the SeibergWitten curves of $S O$ and $S p$ gauge theories from 5-brane geometry [16].

Since an $\mathrm{O6}^{+}$plane has no moduli, the $\mathrm{D}_{4}$ singularity should also have no moduli. In particular, the standard set of moduli associated with resolving the singularity should be lifted. If the $D_{4}$ singularity were purely geometrical, we could certainly resolve it. So the only possible means by which it could be frozen must involve the 3 -form. In 7 dimensions, the two types of orientifold plane $\mathrm{O6}^{-}$and $\mathrm{O6}^{+}$are distinguished by the sign of the contribution of the $\mathbb{R}^{2}$ world-sheets to the string path integral. This sign is related to a discrete choice of NS-NS $B$-field. This discrete $B$-field should 
have its origin in a discrete 3 -form field in $\mathrm{M}$ theory that gives different signs to various contributions to the membrane path integral. Unfortunately, this path integral is not understood, and we do not have a precise geometrical definition of the discrete allowed values of the 3 -form field for a given singularity. We shall return to a discussion of the geometry of the $\mathrm{M}$ theory 3-form later. For now, the primary message is that $\mathrm{M}$ theory appears to have frozen singularities with discrete 3 -form flux. In particular, the 7-dimensional CHL string is described by $\mathrm{M}$ theory on $K 3$ with two frozen $D_{4}$ singularities with some discrete 3-form flux [17].

As this point, let us return to an issue that appeared in our analysis of 7-dimensional orientifolds of section 3.2.4. We found 2 orientifold compactifications with equal numbers of $\mathrm{O6}^{+}$and $\mathrm{O6}^{-}$planes. These configurations are clearly distinct as pertubative string theories. Are they also distinct non-perturbatively? With the strong coupling description of $\mathrm{O6}^{+}$in hand, it seems worth mentioning that one check on the number of non-perturbative configurations is to count the number of non-isomorphic embeddings of the weight lattice for $\left(D_{4}\right)^{4}$ into the $K 3$ lattice, $\Gamma_{3,19}$. If the orientifold configurations are distinct non-perturbatively then it is possible that the difference will be visible in the number of inequivalent ways of embedding $\left(D_{4}\right)^{4}$. There are analogues of this embedding question for lower-dimensional orientifold compactifications. It might be possible to do this counting using the techniques developed in $[57,93] .^{22}$

Now, in order to find $M$ theory compactifications dual to our new 7dimensional theories, for which no orientifold descriptions exist, the lattices discussed in section 2.4 turn out to be very helpful. Recall that the lattice for the CHL string in $d=7$ took the form,

$$
\Lambda_{\mathrm{CHL}}=\Gamma_{3,3} \oplus D_{4} \oplus D_{4}
$$

The orthogonal complement of $\Lambda_{\mathrm{CHL}}$ in the usual $7-d$ heterotic lattice $\Gamma_{3,19}$

\footnotetext{
${ }^{22} \mathrm{~A}$ preliminary computation indeed suggests that there are 2 non-isomorphic embeddings.
} 


\begin{tabular}{|c|c|c|}
\hline Singularity & Discrete Fluxes & Enhanced Gauge Symmetry \\
\hline$D_{4+n}$ & $\mathbb{Z}_{2}$ & $C_{n}$ \\
\hline$E_{6}$ & $\mathbb{Z}_{2}, \mathbb{Z}_{3}$ & $C_{2},\{e\}$ \\
\hline$E_{7}$ & $\mathbb{Z}_{2}, \mathbb{Z}_{3}, \mathbb{Z}_{4}$ & $B_{3}, A_{1},\{e\}$ \\
\hline$E_{8}$ & $\mathbb{Z}_{2}, \mathbb{Z}_{3}, \mathbb{Z}_{4}, \mathbb{Z}_{5}, \mathbb{Z}_{6}$ & $F_{4}, G_{2}, A_{1},\{e\},\{e\}$ \\
\hline
\end{tabular}

Table 14: Singularities, their allowed 3-form fluxes, and enhanced gauge symmetries.

is

$$
\Lambda_{\mathrm{CHL}}^{\perp}=D_{4} \oplus D_{4}
$$

As was already observed in [38], $\Lambda_{\mathrm{CHL}}^{\perp}$ seems to be directly related to the singularities appearing in the dual $\mathrm{M}$ theory description. Indeed, on the heterotic side there are no moduli for $\Lambda_{\mathrm{CHL}}^{\perp}$, and in M theory, the corresponding singularities are frozen. By looking at table 5 , we see that the generalizations of $\Lambda_{\mathrm{CHL}}^{\perp}$ are $E_{6} \oplus E_{6}$ for $\mathbb{Z}_{3}, E_{7} \oplus E_{7}$ for $\mathbb{Z}_{4}, E_{8} \oplus E_{8}$ for the finite groups $\mathbb{Z}_{5}$ and $\mathbb{Z}_{6}$. This leads us to conjecture that these lattices describe frozen singularities in $\mathrm{M}$ theory with the corresponding discrete fluxes. By tuning moduli, we can enhance the unbroken gauge symmetry in the asymmetric orbifold compactification. In the geometric picture, this means that we can enhance certain singularities with flux. This shows that we can also turn on a $\mathbb{Z}_{3}$ flux in an $E_{8}$ singularity. With such a $\mathbb{Z}_{3}$ flux we can resolve the $E_{8}$ into an $E_{6}$, but no further resolution is possible.

A summary of the $A D E$-singularities with their allowed discrete 3 -form fluxes is given in table 14 . Notice that for the $E_{6}$ singularity, we can turn on either a $\mathbb{Z}_{2}$ or a $\mathbb{Z}_{3}$ discrete flux, but not both. In particular, the set of allowed fluxes do not form a group. This is a first sign that these fluxes are classified by a rather intricate geometrical object. In our heterotic string analysis, we always found that the singularities came in pairs. This is presumably related to the fact that on a compact manifold like $K 3$, the total 


\begin{tabular}{|c|c|}
\hline Frozen Singularities & Dual Description \\
\hline$D_{4} \oplus D_{4}$ & $\mathbb{Z}_{2}$ triple \\
\hline$E_{6} \oplus E_{6}$ & $\mathbb{Z}_{3}$ triple \\
\hline$E_{7} \oplus E_{7}$ & $\mathbb{Z}_{4}$ triple \\
\hline$E_{8} \oplus E_{8}$ & $\mathbb{Z}_{5}$ triple \\
\hline$E_{8} \oplus E_{8}$ & $\mathbb{Z}_{6}$ triple \\
\hline$\left(D_{4}\right)^{4}$ & $\mathbb{Z}_{2} \mathrm{~F}$ \\
\hline$\left(E_{6}\right)^{3}$ & $\mathbb{Z}_{3} \mathrm{~F}$ \\
\hline$D_{4} \oplus E_{7} \oplus E_{7}$ & $\mathbb{Z}_{4} \mathrm{~F}$ \\
\hline$D_{4} \oplus E_{6} \oplus E_{8}$ & $\mathbb{Z}_{6} \mathrm{~F}$ \\
\hline
\end{tabular}

Table 15: A list of frozen singularities for 7-d M theory compactifications on $K 3$ with flux. In labeling the dual theories, $G$ triple refers to the $G$ heterotic asymmetric orbifold, while $G \mathrm{~F}$ refers to $\mathrm{F}$ theory on $\left(T^{4} \times S^{1}\right) / G$.

flux must vanish. We therefore always need two singularities with equal fluxes of opposite sign. This appears to be similar to the cancellation of the two Chern-Simons invariants in the $E_{8} \times E_{8}$ heterotic string, and to the gluing conditions for the two del Pezzo surfaces appearing in $\mathrm{F}$ theory descriptions.

It is also natural to ask whether similar dual descriptions exist for the 7-dimensional $\mathrm{F}$ theory compactifications on $\left(T^{4} \times S^{1}\right) / G$. Indeed, in essentially the same way as described above, we are led to a conjectured dual description in terms of $\mathrm{M}$ theory on a $K 3$ surface with a particular set of frozen singularities. The list of frozen singularities for the $M$ theory models dual to asymmetric orbifolds and to these $\mathrm{F}$ theory compactifications appears in table 15 .

If we further compactify $\mathrm{M}$ theory on a singular $K 3$ with discrete 3 -form flux on a circle, we obtain type IIA on the same $K 3$ with RR 3 -form flux. However, we also had a different description of the same theory in terms 
of another orbifold $K 3$ with RR 1-form flux. This has to be a $K 3$ with different geometry because the $K 3$ surfaces that appear with 3 -form flux are in general not orbifold $K 3$ surfaces: they are not quotients of another $K 3$ surface by some discrete group. This picture can therefore only be consistent if there is a duality between IIA on $K 3$ with discrete RR 1-form flux and IIA on a different $K 3$ surface with discrete 3 -form flux.

To argue that such a duality indeed exists, let us examine the charge lattices of both theories obtained by wrapping D0, D2 and D4-branes on the $K 3$. For an orbifold $K 3$ with discrete RR 1-form flux, the charge lattice is constructed as follows: the vanishing cycles in the orbifold $K 3$ generate a lattice $\Lambda_{\text {vanishing }}^{(1)}$. These lattices have been constructed by Nikulin [37] for the $K 3$ case, and appear in table 8. For the case of $X=T^{4}$, the lattice of vanishing cycles appears in table 10 [62]. We should not wrap D-branes over any of these vanishing cycles. This becomes particularly clear by looking at M2-branes wrapping 2-cycles in the M theory description on $\left(X \times S^{1}\right) / G$ (we will elaborate on the connection between the IIA and M theory description in section 4.6.5) . However, we can wrap D2-branes over any cycle orthogonal to $\Lambda_{\text {vanishing }}^{(1)}$, which yields the lattice $\Lambda_{\text {vanishing }}^{(1) \perp}$, the orthogonal complement of $\Lambda_{\text {vanishing }}$ in $\Gamma_{3,19}$. In addition, we can also consider D0 and D4-branes on the orbifold $K 3$, leading to a total charge lattice

$$
\Lambda_{\text {D-branes }}=\Gamma_{1,1} \oplus \Lambda_{\text {vanishing }}^{(1) \perp} .
$$

For $K 3$ surfaces with discrete RR 3-form flux, the charge lattice is constructed in a similar way. However, a new subtlety arises. Namely, not all brane wrappings are allowed. This conclusion follows by applying dualities to the Freed-Witten anomaly [18], which states that the class of the pullback of $H$, the field strength of the NS-NS 2-form $B$, to the D-brane world-volume must vanish. In particular, if the class $[H]$ is torsion of order $k$, only branes that come in stacks of $k$ are allowed since $k[H]=0$ [94]. If we apply this constraint to a D3-brane and further S and T-dualize, we see that the pullback of the 3-form field strength to the D4-brane world-volume has to vanish. In the cases at hand, the field strength of the RR 3 -form will be torsion, say of 
order $k$, which is directly related to the order of the finite group appearing in the 1-form discussion above. Therefore, we can only wrap $D 4$ branes $n k$ times, with $n$ an integer. The contribution to the charge lattice from D0 and D4-branes will therefore not be $\Gamma_{1,1}$, but $\Gamma_{1,1}(k)$. In the 3 -form case, we therefore conclude that the lattice is given by:

$$
\Lambda_{\mathrm{D} \text {-branes }}=\Gamma_{1,1}(k) \oplus \Lambda_{\text {vanishing }}^{(3) \perp}
$$

If the theories with the 1-form and the theories with the 3-form flux are to be dual to each other, the lattices (188) and (189) should be equivalent to each other. Therefore, $\Lambda_{\text {vanishing }}^{(1) \perp}$ has to be an index $k$ sublattice of $\Lambda_{\text {vanishing }}^{(3) \perp}$. This explains how the geometries of the two $K 3$ surfaces can be so different from one another: the lattices of vanishing cycles differ by an element of order $k$. For example, in the case of the CHL string, the lattice $\Lambda_{\text {vanishing }}^{(1) \perp}$ is generated by eight vanishing cycles, and in addition, half of the sum of these cycles is also an element of integral homology. On the other hand, the lattice $\Lambda_{\text {vanishing }}^{(3) \perp}$ is the lattice of two $D_{4}$ singularities. It is also generated by eight vanishing cycles, but this time half the sum of the first four cycles and half the sum of the second set of four cycles each are in integral homology. This lattice is twice as large as $\Lambda_{\text {vanishing }}^{(1) \perp}$, as we expect from the preceeding discussion.

The duality between $K 3$ surfaces with 1-form and 3-form fluxes differs considerably from the usual T-dualities. Typically, T-duality preserves the nature of singularities (since it preserves enhanced gauge symmetries), while in our case, it changes the geometry of the $K 3$ in a much more dramatic way. We note, however, that the $K 3$ surfaces are still birationally equivalent. That this is true can be seen from the $\mathrm{F}$ theory discussion of the previous section. In the construction involving two del Pezzo surfaces, we found singular fibers of type $I_{0}^{*}, I V^{*}, I I I^{*}$ and $I I^{*}$. The corresponding extended Dynkin diagrams are those of $D_{4}, E_{6}, E_{7}$ and $E_{8}$. Birationally equivalent geometrical models of these elliptically fibered $K 3$ surfaces have singularities described by the extended Dynkin diagram minus one node. The singularities of the $K 3$ with 
1-form flux are obtained by removing nodes with Dynkin label $k$ from the singularities. The singularities of the $K 3$ with 3 -form flux are obtained by removing the extended nodes of the Dynkin diagrams, leading to singularities of type $D_{4}, E_{6}, E_{7}$ and $E_{8}$. One can show that the lattices of vanishing cycles obtained this way indeed differ by an element of order $k$, and by construction the $K 3$ surfaces are birationally equivalent.

The novel duality described here raises all kinds of questions. One puzzle that arises is the following: we already described in section 4.4 that type IIA on $K 3 / \mathbb{Z}_{2}$ with a particular choice of 1 -form flux is dual to the CHL string. Naïvely, this string theory appears to be quotient of IIA on $T^{4}$ with a smooth $\mathbb{Z}_{2}$ invariant 1-form i.e., a $\mathbb{Z}_{2}$ invariant element of $H^{1}\left(T^{4}, U(1)\right)$. It is wellestablished that T-duality maps $T^{4}$ with a constant 1 -form to the dual torus with a constant 3 -form. In addition, if the 1 -form is $\mathbb{Z}_{2}$ invariant then so is the 3 -form which is now an element of $H^{3}\left(T^{4}, U(1)\right)$. If we mod out the theory with the 3 -form by the action of the T-dual $\mathbb{Z}_{2}$, we would expect to arrive at IIA on $T^{4} / \mathbb{Z}_{2}$ with a 3 -form flux. This, however, is not consistent with the picture given above.

The reason for this apparent discrepancy is that the $\mathbb{Z}_{2}$ quotient makes sense as a perturbative orbifold only when the vanishing 2-cycles have a halfintegral $B$-field flux [95]. In cases without this flux like geometric orbifolds, we do not have perturbative control over the theory. Therefore, modding out by $\mathbb{Z}_{2}$ yields a duality between IIA on $T^{4} / \mathbb{Z}_{2}$ with 1 and 2-form flux and IIA on $T^{4} / \mathbb{Z}_{2}$ with 2 and 3 -form flux. Neither of these theories has a smooth 7-dimensional limit. On the other hand, we are interested in a duality between IIA on $T^{4} / \mathbb{Z}_{2}$ with only 1-form flux and IIA on another $K 3$ with only 3-form flux. These theories are not perturbative $\mathbb{Z}_{2}$ orbifolds of string theory, and so we cannot naïvely use T-duality to relate them. As we argued above, the duality between these theories is considerably more involved.

However, this still leaves us with the following question: by starting with 
a $T^{4}$ with $\mathbb{Z}_{2}$ invariant 3-form, we can construct the singular manifold $T^{4} / \mathbb{Z}_{2}$ with a non-trivial discrete 3 -form flux. Does string theory compactified on this space make any sense ${ }^{23}$ ? We have not encountered this string theory anywhere in our prior discussion, and we suspect it is inconsistent. It would be nice to give a direct argument for this, perhaps using supersymmetry or anomaly cancellation. A related situation that is worth mentioning arises for type IIA strings: consider an orientifold 6-plane with RR charge $q$. If there is no $B$-field, this plane is supersymmetric for $q \geq-2$. However, with a half-integral discrete $B$-field, the orientifold plane is an $\mathrm{O6}^{+}$plane and is only supersymmetric if $q \geq+2$. This can be seen from a tadpole calculation in string theory, but it is not clear whether there is a direct low-energy argument giving this conclusion.

To summarize our discussion so far: we found 7-dimensional $M$ theory compactifications on $K 3$ with frozen singularities and discrete 3 -form fluxes. These theories are dual descriptions of either 7-dimensional asymmetric heterotic orbifolds or $\mathrm{F}$ theory compactifications. These 7-dimensional theories give 6-dimensional IIA theories by compactifying on a circle, and the 6 and 7dimensional lattices differ by a factor of $\Gamma_{1,1}(k)$. These models serve as data from which we obtain a list of new frozen, or partially frozen, singularities in $M$ theory.

\subsubsection{Three-form flux as equivariant cohomology}

The most natural geometric object with which to identify the $M$ theory 3 -form is a generalized connection on a "2-gerbe"; see, for example, [98]. Although 2-form $B$-fields and "1-gerbes" have a relatively straightforward interpretation in terms of bundles of algebras of operators on infinite-dimensional Hilbert spaces, this description is less clear for the next case of 3 -form fields. For a smooth manifold $X$ we expect that flat 3 -forms are given by the cohomology group $H^{3}(X, U(1))$. There are some indications that for

\footnotetext{
${ }^{23}$ An example involving discrete zero and four form fluxes is discussed in [96, 97].
} 
an orbifold $X / G$ one should again consider the corresponding equivariant group $H_{G}^{3}(X, U(1))$ [88]. It is therefore natural to extend our discussion of equivariant cohomology to this case.

The equivariant cohomology groups $H_{G}^{3}(X, U(1))$ are computable. As explained in appendix $\mathrm{D}$, in the case of a $T^{4} / \mathbb{Z}_{2}$ orbifold we find

$$
H_{\mathbb{Z}_{2}}^{3}\left(T^{4}, U(1)\right) \cong\left(\mathbb{Z}_{2}\right)^{15} .
$$

In terms of the previously introduced generators $\theta^{i}$ and $\xi$, a general element can be written as

$$
C=\sum_{i<j<k} c_{i j k} \theta^{i} \theta^{j} \theta^{k}+\sum_{i<j} c_{i j} \theta^{i} \theta^{j} \xi+\sum_{i} c_{i} \theta^{i} \xi^{2}+c_{0} \xi^{3},
$$

with

$$
c_{i j k}, c_{i j}, c_{i}, c_{0} \in \mathbb{Z}_{2}
$$

Note that we now have contributions of various type. First of all there are obvious 3-forms that come from invariant fields on $T^{4}$. The flat 3-form field on $T^{4}$ are described by $H^{3}\left(T^{4}, U(1)\right)$ and this group is (up to a determinant) canonically isomorphic with $T^{4}$ itself. The $\mathbb{Z}_{2}$ group acts as $C \rightarrow-C$ on the 3 -form field, so the invariant configurations are

$$
C=\sum_{i<j<k} \frac{1}{8 \pi^{2}} C_{i j k} d x^{i} d x^{j} d x^{k}, \quad C_{i j k}=0,1 .
$$

As we explained, there is a well-defined map of the equivariant forms into the invariant forms, and clearly a class of the form (191) gets mapped to the invariant 3-form $C_{i j k}=c_{i j k}$. So the 16 classes $c_{i j k} \theta^{i} \theta^{j} \theta^{k}$ modulo forms of lower degree in the $\theta^{i}$ can be given this geometric interpretation.

The class $\xi^{3}$ is the image of the group cohomology class in $H^{3}\left(\mathbb{Z}_{2}, U(1)\right)$. It describes a trivial 2-gerbe with a non-trivial group action. This is for example the class that describes a non-zero $C$-field in the neighbourhood of the singularity $\mathbb{C}^{2} / \mathbb{Z}_{2}$. It would be an analogue of discrete torsion for the NS $B$-field. The other classes of type $c_{i j} \theta^{i} \theta^{j} \xi$ and $c_{i} \theta^{i} \xi^{2}$ describe mixed cases that are partially geometric and partly group theoretic. 


\subsubsection{Three-form holonomies}

The local computation of the consistent 3-form fluxes is much easier in this case. If we consider the manifold with boundary $X_{0}$ obtained by cutting the 16 singularities out of the $K 3$ manifold $T^{4} / \mathbb{Z}_{2}$, we have again $2^{16}$ possibilities, because at each singularity we have the non-trivial class $\xi^{3}$ coming from $H^{3}\left(\mathbb{Z}_{2}, U(1)\right)$. Now the global topology gives only one condition. Because the boundary $Y=\partial X_{0}$ bounds a four-manifold, the sum of all classes should add up to zero. This reduces the $\left(\mathbb{Z}_{2}\right)^{16}$ to $\left(\mathbb{Z}_{2}\right)^{15}$.

In the same way that flat connections have well-defined holonomies along closed curves of a given homology class, flat 2-gerbes have $U(1)$-valued holonomies along 3 -cycles. The holonomy of the 3 -form $C$ around a fixed point $p$ is determined as follows. The boundary of a neighbourhood of $p$ is given by a 3 -cycle $\Sigma_{p} \cong \mathbb{R}^{3}$ and the holonomy is defined locally as

$$
\exp i \int_{\Sigma_{p}} C=\exp i \pi \varphi(C ; p) .
$$

We propose the following formula for the holonomy of a class $C$ as represented as in (191) around the fixed point $p \in\left(\mathbb{Z}_{2}\right)^{4}$,

$$
\varphi(C ; p)=\sum_{i<j<k} c_{i j k} p^{i} p^{j} p^{k}+\sum_{i<j} c_{i j} p^{i} p^{j}+\sum_{i} c_{i} p^{i}+c_{0},
$$

This formula follows again trivially from the definition of the holonomy as the restriction to the fixed point

$$
i_{p}^{*}(C)=\varphi(C ; p) \xi^{3}
$$

combined with the localisation formula (107) that $i_{p}^{*}\left(\theta^{i}\right)=p^{i} \xi$.

Note that these formulas have a nice algebraic interpretation. Because our generators $\theta^{i}$ satisfy $\left(\theta^{i}\right)^{2}=\theta^{i} \xi$ we have for the variables $p^{i}$ the relation

$$
\left(p^{i}\right)^{2}=p^{i}
$$

This means that we can consider the holonomy as a function of the variables $p^{i}$ over the field $\mathbb{Z}_{2}$ (with $\left.p^{i}=0,1\right)$. 
So quite generally we have the following algebraic interpretation of the holonomy of an equivariant $k$-cocycle $C$ of the torus $T^{n}$. Consider the affine space $A^{n}=\left(\mathbb{Z}_{2}\right)^{n}$ with coordinates $p^{i}$, and let $F\left(A^{n}\right)=\mathbb{Z}_{2}\left[p^{1}, \ldots, p^{n}\right]$ be the space of functions

$$
\varphi: A^{n} \rightarrow \mathbb{Z}_{2}
$$

Then there is a filtration, where $F_{k}\left(A^{n}\right)$ denotes the functions of at most degree $k$. According to the above localisation formula, we can think of the holonomy $\varphi(C ; p)$ as an element of $F_{k}\left(A^{n}\right)$. We can identify the quotients $F_{k}\left(A^{n}\right) / F_{k-1}\left(A^{n}\right)$ with the exterior products $\Lambda^{k}\left(\mathbb{Z}_{2}\right)^{n}$. Clearly the affine group $\left(\mathbb{Z}_{2}\right)^{n} \ltimes S L\left(n, \mathbb{Z}_{2}\right)$ acts on $F\left(A^{n}\right)$ and the orbits form the different types of equivariant classes.

There is an interesting check of the holonomy formula. We can pick a 3 -cycle that is represented by a 3 -plane in $T^{4}$. This will give a 3-cycle on the orbifold $T^{4} / \mathbb{Z}_{2}$ that will divide the 16 fixed points in 2 groups of 8 . Clearly the holonomy of $C$ around that plane should be equal to the sum of the holonomies around the fixed points "to the right" or "to the left" of the cycle. It is not difficult to check that this property is indeed reflected in the above formula. For example, if we pick the plane say spanned by $x^{1}, x^{2}, x^{3}$ the holonomy will be $c_{123}$ and this equals the sum

$$
c_{123}=\sum_{p, p^{4}=0} \varphi(C ; p)=\sum_{p, p^{4}=1} \varphi(C ; p) .
$$

In order to compute the holonomy directly in terms of the $C$-field, we should make use of the formalism of gerbes. Let us consider in all generality an $n$-gerbe defined on a manifold $M$. A connection on this $n$-gerbe is an $(n+1)$-form $C_{i}$, defined over the patches $U_{i}$ of the manifold. The forms over patches $U_{i}$ and $U_{j}$ are related on the non-empty intersection $U_{i j}=U_{i} \cap U_{j}$ by $C_{i}=C_{j}+d C_{i j}$. The $C_{i j}$ can be regarded as connections on $(n-1)$ gerbes defined at the overlap patches $U_{i j}$. The $C_{i j}$ are only defined up to closed forms (with integer periods). On triple intersections $U_{i} \cap U_{j} \cap U_{k}$ there is the consistency condition $d\left(C_{i j}+C_{j k}+C_{k i}\right)=0$ which is compatible with the 
ambiguity of adding closed forms. On the triple overlap, $C_{i j}+C_{j k}+C_{k i}$ and $C_{i j}+C_{j k}+C_{k i}+d C_{i j k}$ define the same cocycle. The $C_{i j k}$ can be thought of as connections on an $(n-2)$-gerbe, defined over the $U_{i j k}=U_{i} \cap U_{j} \cap U_{k}$. This extends all the way until we arrive at zero-forms (functions), which can be thought of as transition functions for a 0 -gerbe (that is, a line bundle).

For a concrete holonomy formula, we do not consider open patches with finite overlap, but reduce the overlaps to infinitesimal size; that is, we cut up $M$ in pieces $M_{i}$ by a partition of unity. If the pieces $M_{i}$ and $M_{j}$ have a common boundary we denote this $M_{i j}$; a common boundary between $M_{i}$, $M_{j}$ and $M_{k}$ is written as $M_{i j k}$ etc. Define $(n-1)$-forms $C_{i}$ over the pieces $M_{i}$; we think about the $M_{i j}$ as the places where we "jump" from one patch to another. The change in connection is given by $C_{i}=C_{j}+d C_{i j} . C_{i j}$ itself is defined over the $M_{i j}$, and has an intrinsic ambiguity by a closed form. One regards $C_{i j}$ as a connection on an $(n-1)$-gerbe over $M_{i j}$ with a $U(1)$ gauge invariance etc.

The concrete holonomy formula is now (note the alternating sign):

$$
\int_{M} C=\sum_{i} \int_{M_{i}} C_{i}-\sum_{i j} \int_{M_{i j}} C_{i j}+\sum_{i j k} \int_{M_{i j k}} C_{i j k}-\ldots .
$$

This is invariant (for $M$ without boundary) under

$$
\begin{aligned}
C_{i} & \rightarrow C_{i}+d L_{i} \\
C_{i j} & \rightarrow C_{i j}+L_{i}+L_{j}+d L_{i j} \\
C_{i j k} & \rightarrow C_{i j k}+L_{i j}+L_{j k}+L_{k i}+d L_{i j k l}
\end{aligned}
$$

Two extreme cases of this formula are for a globally well-defined form $C$, in which case the sum on the r.h.s. reduces to a single term, and the case when only the last sum of integrals in the expression contributes. These are analogues of the bundle case where physicists are used to either using well-defined connections over large patches, or to "putting the holonomy in the transition functions." For the case of connections on gerbes, there is a much larger freedom to "put" the holonomy somewhere. 
As an example, let us return to the 4 -torus $T^{4} \bmod$ the $\mathbb{Z}_{2}$ reflecting all coordinates, where for convenience we now choose coordinates $x$ in $(\mathbb{R} / 2 \mathbb{Z})^{4}$. We label the 16 fixed points of the $\mathbb{Z}_{2}$ by $x^{i}=p^{i}, p \in\left(\mathbb{Z}_{2}\right)^{4}$.

Pick a fixed point $p$ and define a "hyperbox" around this point by the coordinate ranges

$$
p^{1}<x^{1}<p^{1}+\epsilon, \quad p^{i}-\epsilon<x^{i}<p^{i}+\epsilon
$$

for $i=2,3,4$. The hypersurface of this hyperbox is a 3 -surface; we will compute a 3 -form holonomy over it.

Assume the existence of a constant 3-form field $\sum_{i<j<k} c_{i j k} d x^{i} d x^{j} d x^{k} / 8$ in the bulk. From now on we will always assume that the summed over indices are ordered. The first integral in the holonomy formula is over the 2 -faces of the cube; there are 7 of these, of which 6 pair up as opposite faces. The opposite faces do not contribute to the integral, because their contributions cancel. The only contribution comes from the face at $x^{1}=$ $p^{1}+\epsilon$. This gives

$$
\sum_{i} \int_{M_{i}} C_{i}=c_{234} \epsilon^{3}
$$

We next consider the transition functions: these are defined at the edges of $x^{1}=p^{1}, p^{2}<x^{2}<p^{2}-\epsilon, p^{i}-\epsilon<x^{i}<p^{i}+\epsilon$ for $i=3$, 4. The constant 3 -form jumps upon traversing the plane at $x^{1}=p^{1}$ by an amount $c_{i j k} d x^{i} d x^{j} d x^{k} / 4$, and we should write this as $d$ of something. This is inherently ambiguous, so we choose some conventions and write

$$
c_{i j k} d x^{i} d x^{j} d x^{k}=d\left(\sum_{i<j<k} c_{i j k} x^{i} d x^{j} d x^{k}+c_{i j} d x^{i} d x^{j}\right) .
$$

Here the second term is a closed 2-form that does not contribute to the transition function, but will contribute to the holonomy.

We have to integrate this 2-form over the edge of $x^{1}=p^{1}, p^{2}<x^{2}<$ $p^{2}+\epsilon, p^{i}-\epsilon<x^{i}<p^{i}+\epsilon$ for $i=3,4$. Again only one face contributes (the 
one with $\left.x^{1}=p_{1}, x^{2}=p^{2}+\epsilon\right)$, resulting in

$$
\left(c_{134} p_{1} \epsilon+c_{234}\left(p_{2}+\epsilon\right)+c_{34}\right) \epsilon^{2} .
$$

The next transition functions are defined at the edges of the surface $x^{1}=p^{1}$, $x^{2}=p^{2}, p^{i}<x^{3}<p^{3}+\epsilon, p^{4}-\epsilon<x^{4}<p^{4}+\epsilon$. They are

$$
\left(\sum_{i<j<k} c_{i j k} x^{i} x^{j} d x^{k}+\sum_{i<j} c_{i j} x^{i} d x^{j}+c_{i} d x_{i}\right) / 2 .
$$

The integrals result in

$$
\begin{array}{r}
\left(c_{124} p^{1} p^{2}+c_{134} p^{1}\left(p^{3}+\epsilon\right)+c_{234} p^{2}\left(p^{3}+\epsilon\right)\right. \\
\left.+c_{14} p^{1}+c_{24} p^{2}+c_{34}\left(p^{3}+\epsilon\right)+c_{4}\right) \epsilon
\end{array}
$$

The last contribution comes from the point $x^{1}=p^{1}, x^{2}=p^{2}, x^{3}=p^{3}$, $x^{4}=p^{4}+\epsilon$. The transition function is

$$
\left(\sum_{i<j<k} c_{i j k} x^{i} x^{j} x^{k}+\sum_{i<j} c_{i j} x^{i} x^{j}+c_{i} x^{i}+c_{0}\right) / 2 .
$$

Inserting values for the coordinates gives

$$
\begin{array}{r}
c_{123} p^{1} p^{2} p^{3}+c_{124} p^{1} p^{2}\left(p^{4}+\epsilon\right)+c_{134} p^{1} p^{3}\left(p^{4}+\epsilon\right)+c_{234} p^{2} p^{3}\left(p^{4}+\epsilon\right) \\
+c_{12} p^{1} p^{2}+c_{13} p^{1} p^{3}+c_{14} p^{1}\left(p^{4}+\epsilon\right)+c_{23} p^{2} p^{3}+c_{24} p^{2}\left(p^{4}+\epsilon\right) \\
+c_{34} p^{3}\left(p^{4}+\epsilon\right)+c_{1} p^{1}+c_{2} p^{2}+c_{3} p^{3}+c_{4}\left(p^{4}+\epsilon\right)+c_{0}
\end{array}
$$

Adding the contributions (202), (204), (206) and (208) gives formula (195).

\subsubsection{An M theory dual of the CHL string?}

In this section, let us continue to work under the premise that the 3 -form can be treated as a connection on a 2-gerbe. We still expect that there will be further conditions, from equations of motion or anomalies, constraining us to a subset of the choices predicted by equivariant cohomology. If this is the right approach-and this is far from clear to us-then it is interesting 
to study 3 -forms on the global orbifold $T^{4} / \widehat{\mathcal{D}}_{4}$. By $T^{4} / \widehat{\mathcal{D}}_{4}$, we mean the particular global orbifold specified in appendix D.2 with singularities,

$$
D_{4}^{2} \oplus A_{3}^{3} \oplus A_{1}^{2} .
$$

If there is a 3 -form on $T^{4} / \widehat{\mathcal{D}}_{4}$ with local holonomies at the $D_{4}$ singularities only, it would provide a possible candidate $M$ theory dual to the 7 dimensional CHL string.

There is a simple expression for $H_{G}^{3}\left(T^{4}, U(1)\right)$ that suggests that this is indeed possible. Suppose that $T^{4} / G$ has singularities $\oplus G_{i}$, i.e., $A D E$ singularities of type $G_{i}$. Then

$$
H_{G}^{3}\left(T^{4}, U(1)\right) \cong \frac{\oplus_{i} H^{3}\left(G_{i}, U(1)\right)}{H^{3}(G, U(1))} .
$$

Here we have used $H^{3}\left(G_{i}, U(1)\right) \cong \mathbb{Z}_{\left|G_{i}\right|}$. Therefore, there is a natural inclusion

$$
H^{3}\left(G_{i}, U(1)\right) \rightarrow H^{3}(G, U(1))
$$

Equation (209) has a simple interpretation in terms of holonomies of 2gerbes around $S^{3} / G_{i}$. It simply states that the set of equivariant 3-forms is the same as the set of all inequivalent local holonomies, subject only to one overall flux cancellation condition. Thus, all possible holonomies can appear, as long as their sum, viewed as an element of $H^{3}(G, U(1))$, vanishes. In particular, there is a case of $T^{4} / \widehat{\mathcal{D}}_{4}$ with equal and opposite holonomies at the $D_{4}$ singularities, and no holonomies at any of the other singularities. This is precisely what we want for an M theory dual of the CHL string.

In an attempt to make this more precise, we give a tentative expression for the holonomies associated to 3-forms. Points on $T^{4}$ that are invariant under a non-trivial group element lie either on $\mathbb{F}_{2}^{4}$ or on $\mathbb{F}_{3}^{2}$, where $\mathbb{F}_{2}$ and $\mathbb{F}_{3}$ represent the fields of two and three elements. The group $G$ still acts on $\mathbb{F}_{2}^{4}$ and/or $\mathbb{F}_{3}^{2}$. We propose that the holonomy around points on $\mathbb{F}_{2}^{4}$ is given by $G$-invariant cubic polynomials in the four variables $p_{i} \in\{0,1\}$, $i=1,2,3,4$, with coefficients in $\mathbb{Z}_{|G|}$. In the monomials, the highest power 
of $p_{i}$ that can appear is one. To compute the holonomy, we still have to sum over all preimages of a given point in $T^{4} / G$. This can be compared to our discussion of gerbe holonomies for $G=\mathbb{Z}_{2}$ in section 4.6.3. Similarly, the holonomy around points on $\mathbb{F}_{3}^{2}$ is given by $G$-invariant cubic polynomials in the two variables $p_{i} \in\{0,1,2\}, i=1,2$, with coefficients in $\mathbb{Z}_{|G|}$. The highest power or $p_{i}$ appearing in monomials is two. Again a sum over preimages is required. The final answer is to be viewed as an element of $\mathbb{Z}_{|G|}$ in which all local holonomies can embedded.

For example, for $\widehat{\mathcal{D}}_{4}$, the holonomy is given by by a polynomial,

$$
\begin{aligned}
H= & c_{123} p^{1} p^{2} p^{3}+c_{124} p^{1} p^{2} p^{4}+c_{134} p^{1} p^{3} p^{4}+c_{234} p^{2} p^{3} p^{4} \\
& +b_{12} p^{1} p^{2}+b_{13} p^{1} p^{3}+b_{14} p^{1} p^{4}+b_{23} p^{2} p^{3}+b_{24} p^{2} p^{4}+b_{34} p^{3} p^{4} \\
& +a_{1} p^{1}+a_{2} p^{2}+a_{3} p^{3}+a_{4} p^{4}+a_{0}
\end{aligned}
$$

with all coefficients in $\mathbb{Z}_{8}$, and with the entire expression viewed as an element of $\mathbb{Z}_{8}$. In other words, the holonomy will be $\exp (2 \pi i H / 8)$. The group $\widehat{\mathcal{D}}_{4}$ acts on the $p^{i}$. The generator $\beta$ sends $p^{1} \leftrightarrow p^{2}$ and $p^{3} \leftrightarrow p^{4}$, whereas the generator $\alpha$ sends $p^{1} \leftrightarrow p^{3}$ and $p^{2} \leftrightarrow p^{4}$. Imposing $\widehat{\mathcal{D}}_{4}$-invariance on $H$ restricts it to the form

$$
\begin{aligned}
H= & c\left(p^{1} p^{2} p^{3}+p^{1} p^{2} p^{4}+p^{1} p^{3} p^{4}+p^{2} p^{3} p^{4}\right) \\
& +b_{1}\left(p^{1} p^{2}+p^{3} p^{4}\right)+b_{2}\left(p^{1} p^{3}+p^{2} p^{4}\right)+b_{3}\left(p^{1} p^{4}+p^{2} p^{3}\right) \\
& +a\left(p^{1}+p^{2}+p^{3}+p^{4}\right)+a_{0} .
\end{aligned}
$$

Our prescription for the holonomy states that we should sum over preimages. In other words, if $\pi$ denotes the projection $\pi: T^{4} \rightarrow T^{4} / \widehat{\mathcal{D}}_{4}$, the holonomy around a singularity $x \in T^{4} / \widehat{\mathcal{D}}_{4}$ is given by

$$
H(x) \equiv \sum_{p \in \pi^{-1}(x)} H(p) .
$$

One can verify that when $c$ is divisble by two, it will only contribute a multiple of 8 to the holonomy, and therefore $c$ is naturally restricted to $c \in$ $\mathbb{Z}_{2}$. Similarly we find that $b_{i} \in \mathbb{Z}_{4}, a \in \mathbb{Z}_{2}$, and $a_{0} \in \mathbb{Z}_{8}$. These values are in 


\begin{tabular}{|c|c|}
\hline Singularity & Holonomy $\in \mathbb{Z}_{8}$ \\
\hline$D_{4}^{(1)}$ & $a_{0}$ \\
\hline$D_{4}^{(2)}$ & $4 c+2\left(b_{1}+b_{2}+b_{3}\right)+4 a+a_{0}$ \\
\hline$A_{3}^{(1)}$ & $2 b_{2}+4 a+2 a_{0}$ \\
\hline$A_{3}^{(2)}$ & $2 b_{3}+4 a+2 a_{0}$ \\
\hline$A_{3}^{(3)}$ & $2 b_{1}+4 a+2 a_{0}$ \\
\hline$A_{1}^{(1)}$ & $4 c+4\left(b_{1}+b_{2}+b_{3}\right)+4 a+4 a_{0}$ \\
\hline$A_{1}^{(2)}$ & $4 a+4 a_{0}$ \\
\hline
\end{tabular}

Table 16: Three-form holonomies of $T^{4} / \widehat{\mathcal{D}}_{4}$

precise one-to-one correspondence with the groups $H^{p}\left(\widehat{\mathcal{D}}_{4}, H^{3-p}\left(T^{4}, U(1)\right)\right)$ we compute in (260). We can now evaluate the holonomy around each of the singularities in the quotient $T^{4} / \widehat{\mathcal{D}}_{4}$. The result is given in table 16 . If we take $a_{0}=4$ and all other coefficients equal to zero, we find holonomy -1 around the two $D_{4}$ singularities, and vanishing holonomy around all other singularities. We shall explain in section 4.6 .6 why we believe that this particular holonomy is realized, while other possibilities, like choices with holonomy around $A$-type singularities, are unlikely to be consistent. This then appears to be a concrete proposal for the singular $K 3$ with 2 frozen $D_{4}$ singularities that appears in the 7-dimensional $\mathrm{M}$ theory description of the CHL string.

\subsubsection{Some comments on type IIA versus $M$ theory}

One of the puzzles in the duality between type IIA and M theory is that RR fields in type IIA appear to be classified by K-theory, while the M theory 3form requires a quite different framework-perhaps cohomology. A detailed analysis showed that for partition functions computed on large smooth compact spaces, this is not a contradiction [4]. However, a sum over fluxes in $\mathrm{M}$ theory is needed to reproduce the partition function of type IIA string 
theory. One may wonder whether our analysis sheds any light on the relation between $\mathrm{M}$ theory and type IIA. Specifically, let us consider type IIA on $\mathbb{C}^{2} / G$, with RR 1 and 3-form flux. We expect a relation between,

$$
K\left(\mathbb{C}^{2} / G\right) \leftrightarrow H^{*}\left(\left(\mathbb{C}^{2} \times S^{1}\right) / G\right),
$$

or more precisely, a relation between

$$
K_{G}^{0}(p t) /\left(K_{G}^{0}(p t)\right)_{5} \leftrightarrow H^{3}\left(\left(\mathbb{C}^{2} \times S^{1}\right) / G, U(1)\right) .
$$

The action of $G$ on the right is determined by the 1-form flux on the left, i.e., by an element of $K_{G}^{0}(p t) /\left(K_{G}^{0}(p t)\right)_{3}=H^{1}(G, U(1))$. At first sight, the relation (214) appears very problematic. When the 1-form flux is such that $G$ acts faithfully on the $S^{1}$ factor in $\left(\mathbb{C}^{2} \times S^{1}\right) / G$, the latter quotient is smooth and does not have any non-trivial 3-cohomology. Therefore, we cannot detect any phases using a non-trivial Euclidean M2-brane. On the other hand, in type IIA we can consider Euclidean D2-branes, and according to our previous calculations, these pick up phases $\frac{n}{4} \bmod \mathbb{Z}$.

This discrepancy can be traced back to the fact that not all D2-branes in IIA can be lifted to M2-branes in M theory, at least not when there is non-trivial RR 1-form flux in IIA. For example, consider again the case where $G=\mathbb{Z}_{2}$ with non-trivial 1-form flux. The $\mathrm{M}$ theory description is on $\left(\mathbb{C}^{2} \times S^{1}\right) / \mathbb{Z}_{2}$. It is easy to see that there is no M2-brane in this space that reduces to a single D2-brane wrapping $S^{3} / \mathbb{Z}_{2}$ in type IIA. At most we can get two D2-branes wrapping $S^{3} / \mathbb{Z}_{2}$, and these only see twice the 3 -form flux in type IIA.

This mismatch between $M$ theory and type IIA is removed once we take the Freed-Witten anomaly for D-branes into account. The only nonanomalous brane configurations are those for which the NS-NS 2-form field strength $H$ is cohomologically trivial when restricted to the brane worldvolume. Applying S- and T-dualities in a cavalier manner implies that D2-branes are only consistent if the RR 1-form field strength is cohomologically trivial on the D2-brane world-volume. It would be nice to derive this anomaly from a world-volume approach. 
In particular, the D2-brane that wraps $S^{3} / \mathbb{Z}_{2}$ has a non-trivial RR 1form field strength living on its world-volume, and is therefore inconsistent. The 1-form field strength is the non-trivial element of $H^{2}\left(S^{3} / \mathbb{Z}_{2}, \mathbb{Z}\right)=\mathbb{Z}_{2}$ so we can wrap two D2-branes. Therefore, anomaly cancellation removes those D2-branes from the spectrum that do not appear in M theory. It is pleasing to see that anomaly cancellation for D2-branes has such a simple interpretation in $\mathrm{M}$ theory.

The same argument applies more generally: take any D2-brane wrapping some non-trivial three-manifold $B$, and assume it has RR 1-form flux $\left[F^{R R}\right]$. Then there is a circle bundle $E$ over $B$ which is the M theory lift of $B$. This circle bundle has second Chern class $\left[F^{R R}\right]$. However, taking any M2brane in $\mathrm{M}$ theory and projecting it down to $E$ only gives D2-branes if the pullback of the cohomology class of $\left[F^{R R}\right]$ is zero. This follows from the fact that $\left[\pi^{*} F^{R R}\right]$ is cohomologically trivial on $E$, where $\pi: E \rightarrow B$ is the projection. This in turn can be seen from the Gysin exact sequence for sphere fibrations; see, for example, [99]. M theory therefore implements the condition for anomaly cancellation quite generally.

Ultimately, we would like to generalize all this to K-theory, which is the proper setting both for RR fields and for D-branes at small string coupling. We will very briefly discuss a possible generalization of the Freed-Witten anomaly to K-theory. In the spirit of speculative exploration, we shall use S- and T-dualities without worrying about the deep unresolved incompatibilities between K-theory and string dualities. Our hope is to find a conjecture about which configurations are consistent which is natural in the framework of K-theory.

The Freed-Witten anomaly arises in the string path-integral from open Riemann surfaces that end on the D-brane. In the bosonic case, there is a contribution to the path-integral of the form

$$
\exp \left(i \oint_{\partial \Sigma} A\right) \exp i \int_{\Sigma} B
$$

In [18], this is interpretated in the following way: the first term provides a 
trivialization for the second term, viewed as a section of a line bundle over the space of Riemann surfaces that end on the D-brane. Such a trivialization only exists if $H=d B$ is cohomologically trivial when restricted to the Dbrane.

Using S- and T-dualities, we find a corresponding statement for $\mathrm{D}(p-2)-$ branes ending on $\mathrm{D} p$-branes. Let $M$ denote the space on which the $\mathrm{D}(p-2)$ brane is wrapped which ends on a manifold $Q$ on which the $\mathrm{D} p$-brane is wrapped. In the path-integral for the $\mathrm{D}(p-2)$-branes, there is a phase factor

$$
\exp \left(i \oint_{\partial M} \tilde{A}\right) \exp \left(i \int_{M} C^{R R} e^{F+B} \sqrt{\hat{A}}\right)
$$

where $\tilde{A}$ is the dual $(p-2)$-form of the gauge field on the $\mathrm{D} p$-brane, and the second term is the Wess-Zumino term of the $\mathrm{D}(p-2)$-brane action. By analogy with the argument in [18], we interpret the first term as providing a trivialization of the second term, viewed as a section of a line bundle over the space of $\mathrm{D}(p-2)$ branes ending on $Q$. Such a trivialization only exists if the cohomology class of

$$
d\left(C^{R R} e^{F+B} \sqrt{\hat{A}}\right)
$$

restricted to $Q$ vanishes. Taking $B=\sqrt{\hat{A}}=0$ for simplicity, the class $d\left(C^{R R} e^{F}\right)=G^{R R} e^{F}$ restricted to $Q$ should be trivial. In principle we have to sum over all possible topological sectors described by $F$. These will include, for example, bound states of $\mathrm{D}(p-2)$ and $\mathrm{D}(p-4)$-branes ending on $Q$. For each of these separately, the restriction of $G^{R R} e^{F}$ has to vanish, when restricted to $Q$. This will typically imply that $G^{R R}$ itself, restricted to $Q$ has to vanish. Rewriting all this in K-theory language gives a generalization of the Freed-Witten anomaly cancellation condition which we now describe.

Suppose that (in type IIB) the RR field strength $G$ is represented by an element of $K^{1}(Y)$, where $Y$ denotes space-time, and consider a brane represented by an element $Q \in K^{0}(Y)$. Recall that $\mathrm{K}$-theory is a graded ring, and in particular we can form the product $Q . G \in K^{1}(Y)$. We propose the 
following generalization of the Freed-Witten anomaly cancellation condition,

$$
Q \cdot G=0 \text { in } K^{1}(Y)
$$

A similar result applies to type IIA. The product $Q . G$ represents, roughly speaking, the restriction of $G$ to $Q$, which is how it arose in the preceeding discussion.

For Euclidean branes, we have to be quite careful when applying this. First, Euclidean and Minkowski D-branes are apparently classified by different $\mathrm{K}$-theories. If one is classified by $K^{0}$ then the other is classified by $K^{1}$. Secondly, we have to throw out the form of highest degree in $G^{R R}$, since the argument does not apply to that form. This can be accomplished by projecting the appropriate $\mathrm{K}$-theory classes on those that vanish on the $(p+1)$-skeleton for Euclidean $\mathrm{D} p$-branes.

For IIA on $\mathbb{C}^{2} / \mathbb{Z}_{2}$, this implies the following. The product in (215) becomes the product of two elements of $\mathbb{Z}_{4}$, and the projection to forms that vanish on the 3 -skeleton is multiplication by two on $\mathbb{Z}_{4}$. If the $R R$ flux is $1 / 4$, as discussed in section 4.5 , we pick up a phase of $1 / 4$ around $S^{3} / \mathbb{Z}_{2}$. Once we project this phase down to $\mathbb{Z}_{2}$, it remains non-zero, and the corresponding Euclidean brane is inconsistent. We can however take twice $S^{3} / \mathbb{Z}_{2}$, which has phase $1 / 2$ in $\mathbb{Z}_{4}$, and this vanishes after projection. What is the interpretation of this object in $M$ theory? It has exactly the same phase as seen by a Euclidean D0-brane wrapping the non-trivial cycle in $S^{3} / \mathbb{Z}_{2}$, and in $\mathrm{M}$ theory it is therefore described by a "half-momentum" graviton. The worldline of this graviton is the obvious lift of the non-trivial one-cycle in $S^{3} / \mathbb{Z}_{2}$ to $\left(S^{3} \times S^{1}\right) / \mathbb{Z}_{2}$. For $\mathrm{RR}$ flux $1 / 2$, there is no RR 1form flux, and indeed there is never an anomaly. We can therefore directly match the $\mathrm{M}$ theory membranes on $\mathbb{C}^{2} / \mathbb{Z}_{2} \times S^{1}$ with D2-branes on $\mathbb{C}^{2} / \mathbb{Z}_{2}$. Our proposal (215), albeit preliminary, is therefore consistent with M/IIA duality. It would be interesting to develop it in detail. 


\subsubsection{The geometry of the three-form}

The nature of the geometry of the M theory 3 -form is a deep question which is unlikely to have a simple classical answer. It is therefore worth stopping at this point and pondering what we can learn about the 3-form from our results. Our 'experimental' data follows from the classification of allowed discrete fluxes in $A D E$ singularities given in table 14.

There are two natural questions to ask: first, there is a purely local question. Given an $A D E$-singularity of the form $\mathbb{C}^{2} / G$, what are the possible discrete choices of 3-form flux localized at the singularity? The second question is a global one. Given a compact manifold with assorted singularities, are there any relations between the fluxes at different singularities? In all the examples we have considered, the answer to the latter question is that the total flux has to vanish. This is exactly as we would naïvely expect for a compact manifold.

The answer to the first question is much more difficult. One natural candidate is the equivariant 3 -cohomology of $\mathbb{C}^{2} / G$ with values in $U(1)$, which classifies the flat orbifold 2-gerbes. This cohomology group is equal to $H^{3}(G, U(1))=Z_{|G|}$ with $|G|$ the number of elements of $G$. This certainly contains all the fluxes appearing in table 14 , but it is much too large. Nevertheless, it is still possible the physical fluxes are a subset of the choices given by $H^{3}(G, U(1))$. For this to be the case, there must exist additional anomaly constraints or non-linear equations of motion. Non-linear equations might provide an explanation for the lack of any group structure in table 14. The simplest example of a consistency condition that we know is the shifted quantization of the 3-form field strength on curved spaces [31].

Let us consider a related possibility, again in the spirit of speculative exploration. We shall find a picture that seems to reproduce our "data." Although many questions and puzzles remain, it seems worth describing. The key appears to be the study of wrapped branes. Let us consider a 7dimensional $\mathrm{M}$ theory dual of one our heterotic orbifolds. This is $\mathrm{M}$ theory 
on a singular $K 3$ with flux localized at some combination of the singularities. The heterotic string is constructed in $\mathrm{M}$ theory by wrapping an M5-brane on the $K 3$. However, we cannot wrap a single M5-brane because of a generalization of the Freed-Witten anomaly-computed for a single M5-brane in [100]. Let us assume for the moment that equivariant cohomology classifies the possible fluxes, as in the preceeding discussion. If the flux is $N$ torsion, we should wrap $N$ M5-branes. ${ }^{24}$ Is there a bound state of $N$ wrapped M5branes? If not, then this configuration of singularities and fluxes cannot give rise to a heterotic string, and so cannot have a perturbative heterotic dual.

The appearance of bound states of five-branes in terms of irreducible (or connected) covers also appears in the computation of the partition function on $K 3 \times T^{2}$ [101]. Here the bound state of $N$ M5-branes that produces the $U(N) \mathcal{N}=4$ Yang-Mills theory on $K 3$, is obtained by wrapping the fivebranes by an irreducible cover of $T^{2}$. These five-brane configurations are in complete analogy with the long string discussion of [102]. So we expect a bound state if the $K 3$ is locally a $G$-orbifold and $G$ has an $N$-dimensional irreducible representation.

Turning to the local singularity $\mathbb{C}^{2} / G$, we note that the $A_{n}$ series have only 1-dimensional irreducible representations (irreps), while the $D_{n+4}$ series has $n+5$ inequivalent irreps: 4 of dimension 1 and $(n+1)$ of dimension 2 . For $E_{6}$, there are 3 irreps of dimension 1, 3 irreps of dimension 2, and 1 of dimension 3. For $E_{7}$, there are 2 irreps of dimension 1,3 of dimension 2, 2 of dimension 3, and 1 of dimension 4 . Finally for $E_{8}$, there is 1 irrep of dimension 1, 2 irreps of dimension 2, 2 irreps of dimension 3,2 irreps of dimension 4, 1 irrep of dimension 5, and 1 irrep of dimension 6 . The easiest way to determine the list of irreps is from the Dynkin labels of the extended Dynkin diagram for $G$. It is also worth noting (and hardly coincidental!) that these labels are also the starting point for the analysis of triples appearing in $[22,24]$.

${ }^{24}$ This claim involves an extrapolation of known results, but seems quite reasonable. 
Let us now consider the possibilities: take a singularity with a flux of order $k$ (perhaps glued in a $K 3$ ). The above arguments suggest that a wrapped M5-brane is anomalous unless $G$ has an irrep of dimension $k$. Since all irreps for $A_{n}$ are 1-dimensional, we cannot wrap an M5-brane at all on a singularity with non-zero flux. For $D_{n+4}$, there are 2 possibilities. Of all the possible fluxes in the group of equivariant 3 -form fluxes $\mathbb{Z}_{4 n+8}$, only flux of order 1 or order 2 permit the wrapping of an M5-brane. The group of fluxes for $E_{6}$ is $\mathbb{Z}_{24}$. However, there are irreps of dimension 1,2 and 3 only. Therefore, only fluxes of order 1,2 and 3 are allowed. Note that as we desire, this set does not form a group! For $E_{7}$, we have a priori $\mathbb{Z}_{48}$ as the group of choices. Again, only fluxes of order 1,2,3 and 4 are allowed. Finally, for $E_{8}$, we have the group $\mathbb{Z}_{120}$. However, only fluxes of order $1,2,3,4,5$ and 6 are possible. Note also that we can deform an $E_{6}$ singularity, for example, with order 2 flux to a $D_{4}$ singularity with order 2 flux, because this subgroup also has an irrep of dimension 2 . In this way, we completely reproduce the data of table $14 .^{25}$

Let us check which combinations of singularities with flux satisfy our global constraint that the total flux vanish, and which can be embedded in $\Gamma_{3,19}$. The sum of the ranks of the singularities must then be less than 20. To find the minimal list, we discard any deformable singularities. We therefore need not consider $A_{n}$ singularities. With only $D_{4}$, it is clear that we need an even number for flux conservation. The only possibilities are 2 or 4 and both cases are realized in M theory. Analogously, with only $E_{6}$ singularities, we have 2 possibilities: $2 E_{6}$ singularities with opposite $\mathbb{Z}_{3}$ flux, or 3 with $\mathbb{Z}_{3}$ flux. With only $E_{7}$ singularities, there is a single possibility: 2 $E_{7}$ singularities with opposite $\mathbb{Z}_{4}$ flux. With only $E_{8}$ singularities, there are 2 possibilities: $2 E_{8}$ singularities with opposite $\mathbb{Z}_{5}$ or $\mathbb{Z}_{6}$ flux.

\footnotetext{
${ }^{25}$ What we seem to be requiring for an anomaly-free theory can be interpreted via the McKay correspondence. It appears to be the existence of a globally defined reflexive sheaf of rank $k$ for flux of order $k$. For $\mathbb{C}^{2} / G$, the existence of such a sheaf requires that $G$ have an irrep of dimension $k$. Away from the singularity, the sheaf is constructed by taking the trivial $\mathbb{C}^{k}$ bundle over $\mathbb{C}^{2}$ and quotienting by $G$ using the irreducible representation.
} 
We can now consider the mixed cases: for $D_{4}$ with $\mathbb{Z}_{2}$ flux and $E_{6}$ with $\mathbb{Z}_{3}$ flux, we note that a brane must wrap 6 times. To cancel the flux implies one possibility; namely, adding a single $E_{8}$ with $\mathbb{Z}_{6}$ flux. For $D_{4}$ and $E_{7}$, a similar argument requires an additional $E_{7}$. it is not too hard to check that there are no other consistent combinations. This list reproduces table 15.

This argument is natural in the global context where we can see that the resulting $\mathrm{M}$ theory fails to have a perturbative heterotic dual. What seems critical to show is why models which do not permit the wrapping of M5-branes-both local and global models-are inconsistent.

\subsubsection{F theory compactifications with flux}

On the topic of $\mathrm{F}$ theory compactifications with flux, we find no new models. However for completeness, we recall the known results. Starting with $\mathrm{M}$ theory on $K 3$ with 2 frozen $D_{4}$ singularities, we can see from the lattice or from its various dual realizations that there is a non-trivial 8-dimensional limit. This is an $\mathrm{F}$ theory model in which the $2 D_{4}$ singularities are replaced by a frozen $D_{8}$ singularity. This gives an 8-dimensional dual of the CHL string. The second case starts with $\mathrm{M}$ theory on a $K 3$ with 4 frozen $D_{4}$ singularities. This is the model dual to the compactification of the 9-dimensional $(+,-)$ orientifold. Taking its $\mathrm{F}$ theory limit results in a compactification with 2 frozen $D_{8}$ singularities. Both cases were first considered in [17]. These models have been further studied in $[103,104]$.

Can there be any new $\mathrm{F}$ theory models with flux? The 7-dimensional $\mathrm{M}$ theory duals of our heterotic asymmetric orbifolds only lift non-trivially to 8 dimensions for the $\mathbb{Z}_{2}$-triple. This is the first case recalled above. The 7-dimensional $\mathrm{M}$ theory duals of our 7-dimensional $\mathrm{F}$ theory models again only lift non-trivially in 8 dimensions for the case of $\mathbb{Z}_{2}$. However, the $M$ theory dual of the $\mathbb{Z}_{2} \mathrm{~F}$ theory compactification is the second case recalled above with 4 frozen $D_{4}$ singularities. The only other place we might expect to find a new model is from our 6 -dimensional $\mathbb{Z}_{2} \times \mathbb{Z}_{2} \mathrm{~F}$ theory vacuum. This 
model may well have a 6-dimensional $\mathrm{M}$ theory dual with frozen singularities (a precise determination of its lattice is needed to find a candidate model.). However, since the $\mathrm{F}$ theory model without fluxes has no new 7-dimensional limit, we cannot obtain a new $\mathrm{F}$ theory model with flux this way.

\section{Acknowledgements}

We would like to thank P. Aspinwall, P. van Baal, D. Freed, R. Friedman, A. Mikhailov, R. Plesser, N. Saulina, C. Schweigert, G. Segal and E. Witten for helpful conversations. The work of A. K. was partly supported by EU contract HPRN-CT-2000-00122. K. H. was supported in part by NSF-DMS 9709694. J. M. was supported by NSF grants DMS-9704507 and DMS0074126. D. R. M. was supported by the Institute for Advanced Study and by NSF grants DMS-9401447 and DMS-0074072. S. S. was supported in part by the William Keck Foundation and NSF grant PHY-9513835. This work was also supported in part by NSF grant PHY-9907949 at ITP, Santa Barbara.

R. D., J. M., D. R. M. and S. S. would like to thank the organizers of the 1999 I.H.E.S. Summer Workshop for their hospitality, while J. B. and S. S. would also like to thank the organizers of the 1999 Amsterdam workshop for their support. In addition, J. B., K. H., D. R. M. and S. S. would like to thank the Aspen Center for Physics for hospitality both in 1999 and 2000 as this work matured. R. D. and D. R. M. would also like to thank the Institute for Advanced Study for hospitality at various stages of this project. J. B., K. H. and S. S. are also happy to thank the Institute for Theoretical Physics at UCSB for hospitality during the penultimate phase of this project. Last but not least, S. S. would like to thank the Rutgers theory group for their hospitality during the final stage of this project. 


\section{A Lattice Conventions and Some Useful Defini- tions}

In this appendix, we summarize some useful information about lattices and our conventions. A lattice is a free $\mathbb{Z}$-module $L$ equipped with a $\mathbb{Z}$-valued bilinear form $b: L \times L \rightarrow \mathbb{Z}$. The lattice is even if $b(x, x) \in 2 \mathbb{Z}$ for all $x \in L$. In the even case, there is an associated integer-valued quadratic form $q: L \rightarrow \mathbb{Z}$ defined by

$$
q(x)=\frac{1}{2} b(x, x)
$$

We use lattice conventions which agree with those of Lie algebraists rather than those of algebraic geometers. In particular, $A_{n}, D_{n}$, and $E_{n}$ are positive definite even lattices, the root lattices of the corresponding complex semisimple Lie algebras. We use $\Gamma_{k, k}$ to denote the even lattice of rank $2 k$ whose bilinear form has matrix

$$
\left(\begin{array}{cc}
0 & I_{k} \\
I_{k} & 0
\end{array}\right)
$$

We occasionally describe a lattice by directly writing the matrix of the bilinear form in some basis.

If $L$ is a lattice, then $L(n)$ denotes the same lattice with the bilinear form multiplied by $n$ (this is sometimes denoted by $\sqrt{n} L$ ). Thus, $A_{n}(-1)$, $D_{n}(-1)$ and $E_{n}(-1)$ are the negative-definite lattices which appear in algebraic geometry, and $\Gamma_{k, k}(n)$ is the even lattice of rank $2 k$ whose bilinear form has matrix

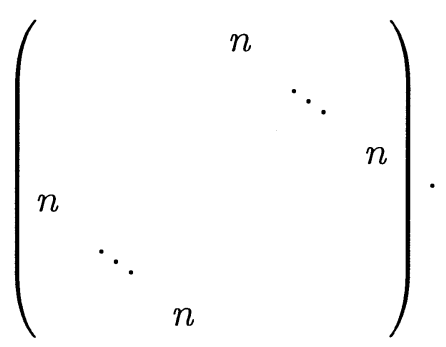

If $x_{1}, \ldots, x_{k} \in L$, then $\left\langle x_{1}, \ldots, x_{k}\right\rangle$ denotes the sublattice of $L$ spanned by $x_{1}, \ldots, x_{k}$. 
Finally, let us review the definition of the discriminant group and discriminant form given by Nikulin [57]. Given any vector $l$ of an integer lattice $L$, we can construct an element of $\operatorname{Hom}(L, \mathbb{Z})=L^{*}$ which acts in the following way: given an $x \in L$, we obtain an integer by evaluating $b(x, l)$. This gives us an injective map from $L \rightarrow L^{*}$. With this canonical embedding of $L$ into $L^{*}$, we can consider the quotient $L^{*} / L$ which is a finite abelian group known as the discriminant group. We can equip this group with a bilinear form $b_{L}$ known as the discriminant form which takes values in $\mathbb{Q} / \mathbb{Z}$,

$$
b_{L}\left(x_{1}+L, x_{2}+L\right)=b\left(x_{1}, x_{2}\right)+\mathbb{Z},
$$

where $x_{1}, x_{2} \in L^{*}$. Under suitable restrictions, this form can determine the isomorphism type of the lattice.

\section{B Heterotic-Heterotic Duality}

\section{B.1 Duality on $S^{1}$}

We will review the argument of [19] and correct a little inaccuracy in that derivation. Consider either of the heterotic string theories compactified on a circle. We write the standard deformation of the heterotic string under the inclusion of a Wilson line a as follows:

$$
W(\mathbf{a})\left(\begin{array}{c}
\mathbf{q} \\
\sqrt{\frac{\alpha^{\prime}}{2}}\left(\frac{n}{r}+\frac{w r}{\alpha^{\prime}}\right) \\
\sqrt{\frac{\alpha^{\prime}}{2}}\left(\frac{n}{r}-\frac{w r}{\alpha^{\prime}}\right)
\end{array}\right)=\left(\begin{array}{c}
\mathbf{q}+w \mathbf{a} \\
\sqrt{\frac{\alpha^{\prime}}{2}}\left(\frac{n-\mathbf{q} \cdot \mathbf{a}-w \mathbf{a}^{2} / 2}{r}+\frac{w r}{\alpha^{\prime}}\right) \\
\sqrt{\frac{\alpha^{\prime}}{2}}\left(\frac{n-\mathbf{q} \cdot \mathbf{a}-w \mathbf{a}^{2} / 2}{r}-\frac{w r}{\alpha^{\prime}}\right)
\end{array}\right) .
$$

The matrix $W(\mathbf{a})$ represents an $S O(17,1)$ rotation acting on the lattice $\Gamma_{17,1}$. To connect the lattice $\Gamma_{17,1}$ to a heterotic string theory, we have to single out a $\Gamma_{1,1}$ sublattice that will be interpreted as the lattice of the spatial momenta $n$ and winding numbers $w$. The complement of $\Gamma_{1,1}$ in $\Gamma_{17,1}$ is then the lattice of vectors $\mathbf{q}$ of the gauge group, which can be $D_{16}^{0, s}$-the composition of the root and spin lattice of $\operatorname{Spin}(32)$-for a $\operatorname{Spin}(32) / \mathbb{Z}_{2}$ theory or $E_{8} \oplus E_{8}$ for the $E_{8} \times E_{8}$ string. Which of these occurs depends 
only on how we choose the $\Gamma_{1,1}$ lattice. Put another way, it depends only on how we choose coordinates for $\Gamma_{17,1}$.

The states of the two heterotic theories are thus connected by a simple coordinate transformation. Consider the vectors $W(\mathbf{a}) \psi$ with $\psi \in \Gamma_{17,1}$, corresponding to the states of the heterotic theory with gauge group $G$ on a circle of radius $R$ with holonomy exp $2 \pi i \mathbf{a}$. Also consider the vectors $W\left(\mathbf{a}^{\prime}\right) \psi^{\prime}$ with $\psi^{\prime} \in \Gamma_{17,1}$ corresponding to the states of the heterotic theory with gauge group $G^{\prime} \neq G$ on a circle of radius $R^{\prime}$ with holonomy $\exp 2 \pi i \mathbf{a}^{\prime}$. We have argued that $W(\mathbf{a}) \psi=U W\left(\mathbf{a}^{\prime}\right) \psi^{\prime}$ with $U$ a coordinate transformation. The mass spectrum of the theories should be preserved by $U$, which therefore should be an element of $O(17) \times O(1)$. The transformations $W(\mathbf{a})$ and $W\left(\mathbf{a}^{\prime}\right)$ allow us to vary all possible continuous parameters of the theories: namely, the holonomies $\mathbf{a}, \mathbf{a}^{\prime}$, and the radii $R, R^{\prime}$. Since the two heterotic theories cannot possibly be continuously connected, the matrix $U$ must correspond to a discrete symmetry. An inversion of all coordinates $n, w$ and $\mathbf{q}_{i}$ corresponds to a parity transformation on the spatial circle, combined with an element of the Weyl group acting on the group lattice. Therefore $U$ is not connected to either the product of the identities of $O(17) \times O(1)$, or to the product of minus the identities. Fixing $U=\operatorname{diag}\left(\mathbf{1}_{16}, 1,-1\right)$, fixes most of the possible choices for $\mathbf{a}$ and $\mathbf{a}^{\prime}$.

A single ansatz fixes most of the remaining freedom. We want to move from a theory with one gauge group to one with another gauge group. This is possible if one of the Kaluza-Klein bosons exchanges roles with one of the 10-dimensional gauge bosons. Explicitly, let a state $\psi$ of the theory with gauge group $G$ with $\mathbf{q}=0$ and non-zero $n, w$ in one theory correspond to a state $\psi^{\prime}$ with non-zero $\mathbf{q}^{\prime}$ and $n^{\prime}=w^{\prime}=0$ in the other theory with gauge group $G^{\prime}$. From the transformation found previously, we can then immediately deduce that $\mathbf{a}^{2}=2$ and that $\mathbf{q}^{\prime}=\mathbf{a}$ : therefore $\mathbf{a}$ is a root of $G^{\prime}$. Furthermore, we find that $\mathbf{a} \cdot \mathbf{a}^{\prime}=-R R^{\prime} / \alpha^{\prime}$. Exchanging the roles of $G$ and $G^{\prime}$ in the ansatz leads to the conclusion that $\mathbf{a}^{\prime}$ is a root of $G$. 
Studying the image of the state $\mathbf{q}=0, n=1, w=0$ tells us that $\alpha^{\prime} / R R^{\prime}$ is an integer, say $k$. Studying the image of the states with $n=w=0$ reveals the consistency condition that $k \mathbf{q} \cdot \mathbf{a}$ be an integer for every $\mathbf{q}$. This implies that $k \mathbf{a}$ is a (co)weight of $G$. In a similar way, we find that $k \mathbf{a}^{\prime}$ is a (co)weight of $G^{\prime}$.

There is no simple way to fix $k$ as there are in fact more solutions. It is clear that $k=0$ will lead to nonsense. Setting $k= \pm 1$ is a possibility, but does not solve our requirements. Both for $\operatorname{Spin}(32)$ and $E_{8} \times E_{8}$, the requirement that $\mathbf{a}$ is a coweight with length $\sqrt{2}$ implies that $\mathbf{a}$ is a root, and therefore inevitably implies that $G=G^{\prime}$, which is not what we desire. It does lead to a duality transformation teaching us that the heterotic theory with group $G$ on a circle of radius $R$ with a trivial holonomy is dual to the same theory on a circle of radius $R^{\prime}=\alpha^{\prime} / R$ with a trivial holonomy, as was already noted in [39].

The next possibility is $k= \pm 2$. This leads to the cases,

- $\mathbf{a}$ is a root of $G^{\prime}$, and $2 \mathbf{a}$ is a coweight of $G$;

- $\mathbf{a}^{\prime}$ is a root of $G$, and $2 \mathbf{a}^{\prime}$ is a coweight of $G^{\prime}$;

- $\mathbf{a} \cdot \mathbf{a}^{\prime}=-\frac{1}{2}$, and $R R^{\prime}=\alpha^{\prime} / 2$.

Solutions to these equations can be found. Given $\mathbf{a}$ and $\mathbf{a}^{\prime}$, the duality transformation is completely fixed and does provide a map from the compactification of one heterotic theory to a compactification of the other. We have not attempted to evaluate the equations for higher $k$.

\section{B.2 Duality on $T^{n}$}

Below 9 dimensions, explicit duality transformations are in general harder to give. In the situations that we will consider, we always choose $g_{i j}=\delta_{i j}$. We will not yet restrict the NS-NS two-form $B_{i j}$. 
The inclusion of a non-zero $B$-field modifies the momenta (6) and (7) in the following way:

$$
\begin{aligned}
\mathbf{k} & =\left(\mathbf{q}+\sum_{i} w_{i} \mathbf{a}_{i}\right) \sqrt{\frac{2}{\alpha^{\prime}}} \\
k_{i L, R} & =\frac{n_{i}-\mathbf{q} \cdot \mathbf{a}_{i}-\sum_{j} \frac{w^{j}}{2}\left(\mathbf{a}_{i} \cdot \mathbf{a}_{j}+2 B_{i j}\right)}{R_{i}} \pm \frac{w_{i} R_{i}}{\alpha^{\prime}} .
\end{aligned}
$$

We will use the following shorthand notation,

$$
\left(\begin{array}{c}
\mathbf{k} \\
k_{i L} \\
k_{i R}
\end{array}\right)=\sqrt{\frac{2}{\alpha^{\prime}}} W\left(\left\{\mathbf{a}_{i}\right\} ; B_{i j}\right)\left(\begin{array}{c}
\mathbf{q} \\
\sqrt{\frac{\alpha^{\prime}}{2}}\left(\frac{n_{i}}{R_{i}}+\frac{w_{i} R_{i}}{\alpha^{\prime}}\right) \\
\sqrt{\frac{\alpha^{\prime}}{2}}\left(\frac{n_{i}}{R_{i}}-\frac{w_{i} R_{i}}{\alpha^{\prime}}\right)
\end{array}\right) .
$$

The explicit expression for $W\left(\left\{\mathbf{a}_{i}\right\} ; B_{i j}\right)$ can be easily deduced or found in [39]. In light of the discussion of the previous subsection, we would like to factorise $W\left(\left\{\mathbf{a}_{i}\right\} ; B_{i j}\right)$ into a number of factors representing contributions that can be traced back to the various directions labelled by $i$. In a gauge theory, this would be possible because of commutativity of the holonomies. In string theory, this seems difficult because the mixing of contributions from various directions by the term quadratic in the $\mathbf{a}_{i}$ and the $B$-field.

However, a little calculation shows that

$$
W\left(\left\{\mathbf{a}_{i}\right\} ; B_{i j}\right) W\left(\left\{\tilde{\mathbf{a}}_{i}\right\} ; \tilde{B}_{i j}\right)=W\left(\left\{\mathbf{a}_{i}+\tilde{\mathbf{a}}_{i}\right\} ; B_{i j}+\tilde{B}_{i j}-\Delta_{i j}\right),
$$

with $\Delta_{i j}$ given by,

$$
\Delta_{i j}=\frac{1}{2}\left(\mathbf{a}_{i} \cdot \tilde{\mathbf{a}}_{j}-\mathbf{a}_{j} \cdot \tilde{\mathbf{a}}_{i}\right) .
$$

Note that the $\Delta_{i j}$ can be appropriately thought of as components of a twoform. These equations are the key to our problem.

Suppose we have a theory with a set of $\mathbf{a}_{i}$, where for one of these, say $\mathbf{a}_{1}$, there exists an $\mathbf{a}_{1}^{\prime}$ such that the pair $\mathbf{a}_{1}, \mathbf{a}_{1}^{\prime}$ has the properties mentioned in the previous subsection. We now want to dualize in the direction labelled by 1 . In the theories under consideration, the $B$-field is a modulus and we can set it to an appropriate value. Set

$$
B_{1 j}=-B_{j 1}=\frac{1}{2}\left(\mathbf{a}_{1} \cdot \mathbf{a}_{j}\right), \quad B_{i j}=0, \quad i, j \neq 1 .
$$


Then the momenta of the theory are given by $W\left(\left\{\mathbf{a}_{i}\right\} ; B_{i j}\right)$ acting on the vectors of the theory with $\mathbf{a}_{i}=B_{i j}=0$. Now with this specific value of the $B$-field, we can write

$$
W\left(\left\{\mathbf{a}_{i}\right\} ; B_{i j}\right)=W\left(\left\{0, \mathbf{a}_{i \neq 1}\right\} ; 0\right) W\left(\left\{\mathbf{a}_{1}, 0\right\} ; 0\right) .
$$

Duality now amounts to replacing $W\left(\left\{\mathbf{a}_{1}, 0\right\} ; 0\right)$ by $U_{1} W\left(\left\{\mathbf{a}_{1}^{\prime}, 0\right\} ; 0\right)$. The matrix $U_{1}$ acts as the identity on all coordinates except for $k_{1 R}$, which is replaced by $-k_{1 R}$. However, $U_{1}$ commutes with $W\left(\left\{0, \mathbf{a}_{i \neq 1}\right\} ; 0\right)$. The holonomies and $B$-field in the dual theory are then summarized as follows,

$$
\begin{aligned}
U_{1} W\left(\left\{\mathbf{a}_{1}^{\prime}, \mathbf{a}_{i \neq 1}\right\} ; B_{i j}^{\prime}\right) \quad \text { with } \quad B_{1 j}^{\prime}=-B_{j 1}^{\prime}=\frac{1}{2}\left(\mathbf{a}_{1}^{\prime} \cdot \mathbf{a}_{j}\right), \\
B_{i j}^{\prime}=0, \quad i, j \neq 1 .
\end{aligned}
$$

The most convenient situation arises when it is possible to choose holonomies so that,

$$
\left(\mathbf{a}_{1} \cdot \mathbf{a}_{j}\right)=\left(\mathbf{a}_{1}^{\prime} \cdot \mathbf{a}_{j}\right)=0 .
$$

Although this is not always the case, we will do this whenever possible.

\section{Classifying Orientifold Configurations}

In this appendix, we classify all possible $T^{n} / \mathbb{Z}_{2}$ orientifolds involving only - and $-^{\prime}$ orientifold planes, for the case $n \leq 5$.

Let $x_{1}, \ldots, x_{n}$ be periodic coordinates for $T^{n}$ with period $2 \pi$ on which the $\mathbb{Z}_{2}$ orientifold group acts by inversion $\left\{x_{\mu}\right\} \mapsto\left\{-x_{\mu}\right\}$. The $2^{n} \mathbb{Z}_{2}$-fixed points are located at $\left\{x_{\mu}\right\}=\left\{a_{\mu} \pi\right\}$ where $a_{\mu}$ are mod 2 integers. We denote this fixed point set by $A_{T^{n}}$ (or $A_{n}$ or just $A$ if there is no chance of confusion). We represent the distribution of $\mathrm{O}^{-{ }^{-}}$planes by a function on $A$ with values in the integers $\bmod 2$,

$$
D: A \rightarrow\{0,1\}
$$

The function simply counts the $(\bmod 2)$ number of D-branes at the fixed points. Namely, $D(a)=0$ if $a$ is $\mathrm{O}^{-}$and $D(a)=1$ if $a$ is an $\mathrm{O}^{{ }^{\prime}}$ plane. The 
consistency conditions on the distribution are stated easily in a T-dual picture, where the locations of $32 \mathrm{D}$-branes are represented by the $O(32)$ Wilson lines on the dual torus $\widehat{T}^{n}$. Let us denote the coordinates of the dual torus by $\widehat{x}^{1}, \ldots, \widehat{x}^{n}$. A single $\mathrm{D}(9-n)$-brane at the fixed point $\left\{x_{\mu}\right\}=\left\{a_{\mu} \pi\right\}$ is represented by the flat real line bundle $\xi(a)$ on $\widehat{T}^{n}$ which has holonomy $(-1)^{a_{\mu}}$ in the $\widehat{x}^{\mu}$-direction. Therefore, a distribution $D$ of $\mathrm{D}$-branes is represented by the flat bundle

$$
\xi(D)=\bigoplus_{a \in A} \xi(a)^{\oplus D(a)}
$$

Since there are exactly $32 \mathrm{D}(9-n)$-branes, the number of fixed points with $D=1$ must be even and also must be less than or equal to 32 . This latter condition is vacuous as long as $n \leq 5$. There is a further condition on the distribution $D$ that

$$
w_{1}(\xi(D))=w_{2}(\xi(D))=C S(\xi(D))=0 .
$$

The vanishing of $w_{1}$ and $w_{2}$ is required since the $O(32)$ bundle must lift to a Spin(32) bundle. The condition $C S(\xi(D))=0$ means the vanishing of the Chern-Simons invariant of the flat Spin(32) bundle. This is required from heterotic world-sheet anomaly cancellation as discussed in section 2.2.

To determine the allowed distributions, it is useful to introduce the notion of reduction of a distribution $D$ along circles. Let $T^{1} \subset T^{n}$ be a circle subgroup where $T^{n}$ is regarded as an abelian group defined by addition in $\left\{x_{\mu}\right\}$. Then $T^{1}$ is invariant under $\mathbb{Z}_{2}$ and contains exactly two $\mathbb{Z}_{2}$ fixed points; the origin 0 and the midpoint $a_{T^{1}}$. The $\mathbb{Z}_{2}$ action on $T^{n}$ descends to a $\mathbb{Z}_{2}$ action on $T^{n} / T^{1} \cong T^{n-1}$. The fixed point set $A_{T^{n}} / T^{1}$ is the quotient of $A_{T^{n}}$ by shifts by $a_{T^{1}}$. Now let us define a function $\bar{D}_{T^{1}}: A_{T^{n}} / T^{1} \rightarrow\{0,1\}$ by the average

$$
\bar{D}_{T^{1}}([a])=D(a)+D\left(a+a_{T^{1}}\right) .
$$

We call this the reduction of $D$ along $T^{1}$. We note that $T^{n} / T^{1}$ is dual to the subtorus $\widehat{T}^{n-1} \subset \widehat{T}^{n}$ orthogonal to $T^{1}$. It is easy to see that the bundle 
corresponding to $\bar{D}_{T^{1}}$ is equal to the restriction of $\xi(D)$ on this subtorus,

$$
\xi\left(\bar{D}_{T^{1}}\right)=\left.\xi(D)\right|_{\widehat{T}^{n-1}} .
$$

Namely, reduction of $D$ along $T^{1}$ corresponds to a restriction of $\xi(D)$ on the subtorus $\widehat{T}^{n-1}$ orthogonal to $T^{1}$. For a sufficiently large $n$, a distribution $D$ satisfies the conditions (224) if and only if its reduction on an arbitrary subgroup $T^{1} \subset T^{n}$ satisfies (224). To be precise, this is true when $w_{1}=0$ for $n \geq 2$, when $w_{2}=0$ for $n \geq 3$, and when $C S=0$ for $n \geq 4$. In what follows, we make use of this fact and inductively determine the allowed distributions starting from small $n$.

\section{C.1 Configurations on $T^{2}$}

We first determine the allowed distributions on $T^{2}$ where there are four $\mathbb{Z}_{2}$ fixed points $\left(a_{1}, a_{2}\right)=(0,0),(0,1),(1,0)$, and $(1,1)$. The condition $C S=0$ is vacuous in this case and we only need require the topological conditions $w_{1}=w_{2}=0$. It is useful to note that the total Stiefel-Whitney class of $\xi(a)$ is $w(\xi(a))=1+a_{1} \theta_{1}+a_{2} \theta_{2}$ where $\theta_{1}$ and $\theta_{2}$ are generators of $H^{1}\left(T^{2}, \mathbb{Z}_{2}\right) \cong \mathbb{Z}_{2} \oplus \mathbb{Z}_{2}$. Using the product formula of the total StiefelWhitney class [105] we find $w(\xi(D))=\prod_{a \in A_{2}}\left(1+a_{1} \theta_{1}+a_{2} \theta_{2}\right)^{D(a)}$. From this, it is easy to see that only $D \equiv 0$ is allowed. For example, if $D \equiv 1$ (all four are $\left.\mathrm{O}^{{ }^{\prime}}\right)$, we have $w=1 \cdot\left(1+\theta_{1}\right)\left(1+\theta_{2}\right)\left(1+\theta_{1}+\theta_{2}\right)=1+\theta_{1} \theta_{2}$ and we find $w_{2} \neq 0$. If $D$ is not constant $D \not \equiv 0,1$ (mixture of $\mathrm{O}^{-}$and $\mathrm{O}^{{ }^{\prime}}$ ), we do not even have $w_{1}=0$. Thus, $D \equiv 0$ (all 4 are $\mathrm{O}^{-}$) is the only allowed distribution.

\section{C.2 Configurations on $T^{3}$}

We move on to $T^{3}$ where there are 8 fixed points. The reduction along a circle must be an allowed distribution in $T^{2}$, which is identically zero as we have seen above. Thus, $\bar{D}_{T^{1}} \equiv 0$ for any $T^{1} \subset T^{3}$. By taking $T^{1}$ as the circle in the first entry, we obtain $D\left(a_{1}, a_{2}, a_{3}\right)+D\left(a_{1}+1, a_{2}, a_{3}\right)=0 \bmod$ 
2. Likewise we obtain similar conditions along the shift in the second and the third entries. We therefore have

$$
\begin{gathered}
D\left(a_{1}+1, a_{2}, a_{3}\right)=D\left(a_{1}, a_{2}+1, a_{3}\right)= \\
D\left(a_{1}, a_{2}, a_{3}+1\right)=D\left(a_{1}, a_{2}, a_{3}\right) \bmod 2 .
\end{gathered}
$$

Namely, $D$ is a constant function on $A_{3}$. There are two possibilities: $D \equiv 0$ and so all 8 are $\mathrm{O}^{-}$, or $D \equiv 1$ and all 8 are $\mathrm{O}^{-{ }^{\prime}}$. Since $n=3<4$, we must still impose the condition $C S=0$. It is obvious that $C S=0$ for $D \equiv 0$ but, as noted before, for $D \equiv 1$ we have $C S \neq 0$. So only $D \equiv 0$ (all 8 are $\mathrm{O}^{-}$) is an allowed distribution.

\section{C.3 Configurations on $T^{4}$}

We next consider $T^{4}$ where there are 16 fixed points. Since the allowed distribution in the $T^{3}$ case is identically zero (all 8 are $\mathrm{O}^{-}$) as we have just seen, the reduction of $D$ along any circle subgroup must be identically zero. As in $T^{3}$ case, this means that $D$ must be a constant function on $A_{4}$. Since $n=4$, this is sufficient for $w_{1}=w_{2}=C S=0$ to be obeyed. Thus, $D \equiv 0$ (all 16 are - ) and $D \equiv 1$ (all 16 are $-^{\prime}$ ) are the allowed distributions.

\section{C.4 Configurations on $T^{5}$}

We finally consider $T^{5}$ where there are 32 fixed points. $D \equiv 0$ (all 32 are $\mathrm{O}^{-}$) and $D \equiv 1$ (all 32 are $\mathrm{O}^{-^{\prime}}$ ) are allowed dsitributions since, for these cases, the reduction along any circle is identically zerQ which is an allowed distribution in $T^{4}$. In addition

$$
\left\{\begin{array} { l } 
{ D ( 0 , a _ { ( 4 ) } ) = 0 } \\
{ D ( 1 , a _ { ( 4 ) } ) = 1 }
\end{array} \quad \forall a _ { ( 4 ) } \in A _ { 4 } , \quad \text { and } \quad \left\{\begin{array}{l}
D\left(0, a_{(4)}\right)=1 \\
D\left(1, a_{(4)}\right)=0
\end{array} \quad \forall a_{(4)} \in A_{4}\right.\right.
$$

are also allowed distributions. To see this, let $T^{1}$ be a circle subgroup of $T^{5}$ and let $a_{T^{1}}$ be the $\mathbb{Z}_{2}$ invariant midpoint of $T^{1}$. Then, it is easy to see that $\bar{D}_{T^{1}} \equiv 0$ if $a_{T^{1}}=(0, \ldots)$ and $\bar{D}_{T^{1}} \equiv 1$ if $a_{T^{1}}=(1, \ldots)$. Thus, the reduction along an arbitrary cricle is an allowed distribution in $T^{3}$ and therefore 
(227) is indeed allowed. We show below that an alloweil distribution, if not identically 0 or 1 , must be of this type up to a coordinate transformation.

Suppose $D$ is an allowed distribution which is not identically 0 or 1 . This means that the reduction along some $x_{\mu}$ is identically 1 . We may assume that this is true in the $x_{5}$ direction. By a change of coordinate, if necessary, we may also assume that the reduction along $x_{4}$ is identically 0 . The fixed point set of $T^{3}$ then has a disjoint partition into two components $\tilde{A}_{3}$ and $\tilde{\tilde{A}}_{3}$, where $A_{3}=\tilde{A}_{3} \cup \tilde{\tilde{A}}_{3}$ and $\tilde{A}_{3} \cap \tilde{\tilde{A}}_{3}=\emptyset$. Then the $\mathrm{O}^{{ }^{\prime}}$ planes, which are located where $D=1$, are found at $\left(\tilde{a}_{(3)}, 0,0\right),\left(\tilde{a}_{(3)}, 1,0\right),\left(\tilde{\tilde{a}}_{(3)}, 0,1\right)$, and $\left(\tilde{\tilde{a}}_{(3)}, 1, \pi\right)$. We let $\tilde{a}_{(3)}$ and $\tilde{\tilde{a}}_{(3)}$ run over the disjoint partition $\tilde{A}_{3}$ and $\tilde{\tilde{A}}_{3}$, respectively.

Now let us redefine coordinates so that $x_{1,2,3}^{\prime}=x_{1,2,3}+x_{4}$ and $x_{4,5}^{\prime}=x_{4,5}$. Ir these new coordinates, the $\mathrm{O}^{{ }^{-}}$planes are at $\left(\tilde{a}_{(3)}, 0,0\right),\left(\tilde{a}_{(3)}+1^{3}, 1,0\right)$, $\left(\tilde{\tilde{a}}_{(3)}, 0,1\right)$, and $\left(\tilde{\tilde{a}}_{(3)}+1^{3}, 1,1\right)$, where $1^{3}=(1,1,1)$. Let $T^{1}$ be the circle in the $x_{4}^{\prime}$ direction. 'The reduction $\bar{D}_{T^{1}}$ must be identicaliy 0 or 1 . It is easy to see that $\tilde{A}_{3}+1^{3}=\tilde{A}_{3}$ and $\tilde{\tilde{A}}_{3}+1^{3}=\tilde{\tilde{A}}_{3}$ if $\bar{D}_{T^{1}} \equiv 0$, whereas $\tilde{A}_{3}+1^{3}=\tilde{\tilde{A}}_{3}$ if $\bar{D}_{T^{2}} \equiv 1$. We discuss these cases separately.

- For $\bar{D}_{T^{1}} \equiv 0$, both $\tilde{A}_{3}$ and $\tilde{\tilde{A}}_{3}$ are invariant under shift by $1^{3}$. There are five possibilities. $\# \widetilde{A}_{3}=0,2,4,6$, or 8. If \# $\hat{A}_{3}=0$, all $16 \mathrm{O}^{-}$ have $x^{5}=1$ arid $D$ is of type (227). Similarly for the case $\# \tilde{A}_{3}=$ 8. If $\# \widetilde{A}_{3}=2$ or 6 , the reduction of $D$ along $x^{1}$ is not a constant function and therefore $D$ is not allowed. Finally let us consider the case \# $\tilde{A}_{3}==4$. The set $\tilde{A}_{3}$ must be of the type $\tilde{A}_{3}=\{(0, a, b),(1, a+$ $\mathrm{I}, b+1),(0, c, d),(1, c+1, d+1)\}$ with $(c, d) \neq(a, b)$. If $(c, d)=(a+1, b)$, $x^{1}+x^{3}+x^{5}=b$ at all $16 \mathrm{O}^{-}$. If $(c, d)=(a, b+1), x^{1}+x^{2}+x^{5}=a$ at all $16 \mathrm{O}^{-^{\prime}}$. If $(c, d)=(a+1, b+1)$, then $x^{2}+x^{3}+x^{5}=a+b$ at all $16 \mathrm{O}^{\mathrm{L}^{\prime}}$. 'Therefore for every case, the distribution $D$ is of type (227) for a suitable choice of coordinates.

- For $\bar{D}_{T^{1}} \equiv 1, \tilde{A}_{3}$ and $\tilde{A}_{3}+1^{3}$ determine a partition of $A_{3}$ and therefore $\tilde{A}_{3}$ consists of four points. In the coordinate system $x_{1,2,3}^{\prime \prime}=x_{1,2,3}+x_{5}$, 


\begin{tabular}{|c|l|}
\hline Dimension & Allowed Configurations \\
\hline$T^{2}$ & $4 \mathrm{O}^{-}$ \\
\hline$T^{3}$ & $8 \mathrm{O}^{-}$ \\
\hline$T^{4}$ & $16 \mathrm{O}^{-}$ \\
& $16 \mathrm{O}^{-\prime}$ \\
\hline$T^{5}$ & $32 \mathrm{O}^{-}$ \\
& $32 \mathrm{O}^{-}$ \\
& $16 \mathrm{O}^{-}+16 \mathrm{O}^{-}{ }^{-}\{$as in $(227)\}$ \\
\hline
\end{tabular}

Table 17: Allowed configurations of $O p^{-}$and $O p^{-1}$ planes.

$x_{4,5}^{\prime \prime}=x_{4,5}$, the $\mathrm{O}^{-^{\prime}}$ planes are at $\left(\tilde{a}_{(3)}, 0,0\right),\left(\tilde{a}_{(3)}, 1,0\right),\left(\tilde{a}_{(3)}, 0,1\right)$, and $\left(\tilde{a}_{(3)}, 1,1\right)$. If the reduction in the $x_{1}^{\prime \prime}$ direction is identically zero, $\widetilde{A}_{3}$ consists of four points $(0, a, b),(1, a, b),(0, c, d)$ and $(1, c, d)$ where $(c, d)=(a, b+1)$ or $(a+1, b)$. In the former case, $x_{2}^{\prime \prime}=a$ for all four points (and therefore at all $16 \mathrm{O}^{{ }^{\prime}}$ in $T^{5}$ ) while $x_{3}^{\prime \prime}=b$ at all 16 $\mathrm{O}^{{ }^{\prime}}$ in the latter case. Thus, in both cases $D$ is of type (227). If the reduction in the $x_{1}^{\prime \prime}$ direction is identically $1, \widetilde{A}_{3}$ consists of $(a, 0,0)$, $(a, 1,1),(b, 0,1)$ and $(b, 1,0)$ where $a=b$ or $a=b+1$. In the case $a=b, x_{1}^{\prime \prime}=a$ at all $16 \mathrm{O}^{{ }^{\prime}}$ while $x_{1}^{\prime \prime}+x_{2}^{\prime \prime}+x_{3}^{\prime \prime}=a$ at all $16 \mathrm{O}^{{ }^{\prime}}$ in the case $a=b+1$. Therefore in both cases $D$ is of type (227).

In summary, we have shown that an allowed distribution $D$ with $D \not \equiv 0,1$ must be of type (227). The results obtained in this appendix are compiled in table 17 which lists the allowed distributions for $T^{n}$ with $2 \leq n \leq 5$. 


\section{Equivariant Bundles and Equivariant Cohomol- ogy}

\section{D.1 Equivariant cohomology via spectral sequences}

Vector bundles over an orbifold $X / G$ are best described in terms of equivariant bundles over the cover $X$. Equivariant bundles are bundles $E \rightarrow X$ where the action of the group $G$ on the base is given a lift to the fibers. Geometrically an equivariant bundle can be seen as a bundle over the 'homotopy quotient' $X_{G}$. This is a smooth space that is homotopy equivalent to $X / G$. It is a fiber bundle over the classifying space $B G$ with fiber $X$. By definition, there is a principal $G$-bundle $E G$ over the classifying space whose total space is contractible, and the homotopy quotient $X_{G}$ is defined as the associated bundle

$$
X_{G}=(E G \times X) / G
$$

Both $B G$ and $X_{G}$ are in general infinite-dimensional spaces. The equivariant cohomology $H_{G}^{*}(X)$ is by definition the cohomology of the space $X_{G}$

$$
H_{G}^{*}(X)=H^{*}\left(X_{G}\right)
$$

Note that as a special case when $X$ is a point (or when $X$ is contractible) we obtain the group cohomology of $G$, which (in the discrete topology) is defined as the cohomology of the classifying space

$$
H^{*}(G)=H^{*}(B G)=H_{G}^{*}(p t)
$$

Since we have a bundle $X_{G} \rightarrow B G$ with fiber $X$, the cohomology of $X_{G}$ can be computed by spectral sequence techniques from the cohomology of $X$ and $B G$. The fibration also gives us some useful maps. In particular, there is a map

$$
H^{*}(B G) \rightarrow H_{G}^{*}(X)
$$

that maps the group cohomology into the equivariant cohomology. This makes the equivariant cohomology a module for $H^{*}(B G)$. There is also an 
inclusion of the fiber $X$ in the space $X_{G}$ and this gives a map $H^{*}\left(X_{G}\right) \rightarrow$ $H^{*}(X)$. Since the image is obviously $G$-invariant, we have in general a map of the equivariant cohomology of $X$ onto the invariant cohomology of $X$

$$
H_{G}^{*}(X) \rightarrow H^{*}(X)^{G}
$$

So, there is a canonical way to associate an invariant form to an equivariant form, but in general there is no opposite map. That is, if we are given an invariant form there is no unique equivariant representative.

We will give two examples of group cohomologies that will be relevant for this paper. First, for $G=\mathbb{Z}_{2}$ the classifying space $B \mathbb{Z}_{2}$ can be chosen to be the infinite real projective space $\mathbb{R}^{\infty}$. This has the non-vanishing cohomology groups

$$
H^{2 k}\left(\mathbb{R P}^{\infty}, \mathbb{Z}\right) \cong \mathbb{Z}_{2}, \quad k>0
$$

We can write this succinctly as

$$
H^{*}\left(\mathbb{R} \mathbb{P}^{\infty}, \mathbb{Z}\right) \cong \mathbb{Z}[y] /(2 y)
$$

where $y$ is a generator of degree two that satisfies $2 y=0$. We will also need below the cohomology with twisted coefficients $\widetilde{\mathbb{Z}}$, where we twist the module $\mathbb{Z}$ with the non-trivial representation of $\mathbb{Z}_{2}$. Then we find

$$
H^{2 k-1}\left(\mathbb{R} \mathbb{P}^{\infty}, \widetilde{\mathbb{Z}}\right) \cong \mathbb{Z}_{2}, \quad k>0 .
$$

or, with $\xi$ a generator of degree 1 ,

$$
H^{*}\left(\mathbb{R} \mathbb{P}^{\infty}, \widetilde{\mathbb{Z}}\right) \cong \xi \mathbb{Z}[y] /(2 y, 2 \xi)
$$

With $\mathbb{Z}_{2}$ coefficients we get a particular simple result

$$
H^{*}\left(\mathbb{R P}^{\infty}, \mathbb{Z}_{2}\right) \cong \mathbb{Z}_{2}[\xi]
$$

where now $\xi$ is a generator of degree one. This results generalizes to cyclic groups. 
Another important generalization of relevance to this paper is the case where $G$ is a finite subgroup of $S U(2)$. These groups are of $A D E$-type. In that case we find

$$
H^{2+4 k}(B G, \mathbb{Z}) \cong \mathbb{Z}_{p}, \quad k \geq 0
$$

where $\mathbb{Z}_{p}=G /[G, G]$ is the abelianization of $G$, i.e., for the binary dihedral group of order $4 n$ denoted $\widehat{\mathcal{D}}_{n}$, there are two cases: $\mathbb{Z}_{2} \times \mathbb{Z}_{2}$ for even $n$ and $\mathbb{Z}_{4}$ for odd $n$. The remaining cases are $\mathbb{Z}_{3}$ for the binary tetrahedral subgroup $\mathbb{T}$ which gives an $E_{6}$ singularity, $\mathbb{Z}_{2}$ for the binary octahedral subgroup $\mathbb{O}$ which gives an $E_{7}$ singularity, and trivial for the icosahedral subgroup $\mathbb{I}$ which gives an $E_{8}$ singularity. The other non-vanishing cohomology groups are

$$
H^{4 k}(B G, \mathbb{Z}) \cong \mathbb{Z}_{|G|}, \quad k>0
$$

As an example we will compute the equivariant cohomology of $T^{4}$ with the $\mathbb{Z}_{2}$ action $x \rightarrow-x$ (thanks to $\mathrm{D}$. Freed). The cohomology of $T^{4}$ is generated by the classes $\eta^{i}$ of degree 1 that satisfy $\left(y^{i}\right)^{2}=0$. We can think of them as the images of the 1-forms $d x^{i}$ in integer cohomology. So we find

$$
H^{*}\left(T^{4}, \mathbb{Z}\right) \cong \mathbb{Z}\left[\eta^{1}, \eta^{2}, \eta^{3}, \eta^{4}\right] /\left(\left(\eta^{i}\right)^{2}\right)
$$

Now the $E_{2}$ tem in the spectral sequence is

$$
E_{2}^{p, q}=H^{p}\left(\mathbb{R} \mathbb{P}^{\infty}, H^{q}\left(T^{4}, \mathbb{Z}\right)\right)
$$

Now we have to decompose the $\mathbb{Z}_{2}$ module $H^{q}\left(T^{4}\right)$ in irreducible representations. This is quite simple because we get the trivial representation $\mathbb{Z}$ for $q$ even, and the non-trivial representation $\widetilde{\mathbb{Z}}$ for $q$ odd. This gives the following 
generators for $E_{2}^{p, q}$ (all free indices are ordered $i<j<k$ etc)

\begin{tabular}{|c|cccccc|}
\hline 4 & $\eta^{1} \eta^{2} \eta^{3} \eta^{4}$ & 0 & & & & \\
3 & 0 & $\xi \eta^{i} \eta^{j} \eta^{k}$ & 0 & & & \\
2 & $\eta^{i} \eta^{j}$ & 0 & $\eta^{i} \eta^{j} y$ & 0 & & \\
1 & 0 & $\xi \eta^{i}$ & 0 & $\xi \eta^{i} y$ & 0 & \\
0 & $\mathbb{Z}$ & 0 & $y$ & 0 & $y^{2}$ & 0 \\
\hline$q / p$ & 0 & 1 & 2 & 3 & 4 & 5 \\
\hline
\end{tabular}

Since there is no cohomology in odd degree the differential is trivial and $E_{2}$ equals the $E_{\infty}$ term. There is a further extension problem that has to be solved to find the actual cohomology groups, but turns out to be trivial too. The result is that the non-trivial groups are

$$
H_{\mathbb{Z}_{2}}^{2}\left(T^{4}, \mathbb{Z}\right) \cong \mathbb{Z}^{6} \oplus\left(\mathbb{Z}_{2}\right)^{5}
$$

and

$$
H_{\mathbb{Z}_{2}}^{4}\left(T^{4}, \mathbb{Z}\right) \cong \mathbb{Z} \oplus\left(\mathbb{Z}_{2}\right)^{15}
$$

Since the odd cohomology vanishes we find that

$$
H_{\mathbb{Z}_{2}}^{1}\left(T^{4}, U(1)\right) \cong\left(\mathbb{Z}_{2}\right)^{5}, \quad \text { and } \quad H_{\mathbb{Z}_{2}}^{3}\left(T^{4}, U(1)\right) \cong\left(\mathbb{Z}_{2}\right)^{15}
$$

\section{D.2 The case of $T^{4} / G$ for more general $G$}

We now briefly describe the generalization of our results for $\dot{T}^{4} / \mathbb{Z}_{2}$ to certain other quotients of four-tori. The possible groups which can act on $T^{4}$ giving a Calabi-Yau are $G=\mathbb{Z}_{3}, \mathbb{Z}_{4}, \mathbb{Z}_{6}, \widehat{\mathcal{D}}_{4}, \widehat{\mathcal{D}}_{5}, \mathbb{T}[56]$. For $\widehat{\mathcal{D}}_{4}$ and $\mathbb{T}$ (the binary tetrahedral group), there are actually multiple possible actions which result in different singularities for $T^{4} / G$. We list these possibilities in table 18 .

We shall restrict to the specific cases of $G=\mathbb{Z}_{3}, \mathbb{Z}_{4}, \mathbb{Z}_{6}, \widehat{\mathcal{D}}_{4}, \widehat{\mathcal{D}}_{5}$. The particular $\widehat{\mathcal{D}}_{4}$ quotient to be described below results in a $T^{4} / G$ with singularities,

$$
D_{4}^{2} \oplus A_{3}^{3} \oplus A_{1}^{2}
$$




\begin{tabular}{|c|c|}
\hline Space & Singularities \\
\hline$T^{4} / \mathbb{Z}_{2}$ & $A_{1}^{16}$ \\
\hline$T^{4} / \mathbb{Z}_{3}$ & $A_{2}^{9}$ \\
\hline$T^{4} / \mathbb{Z}_{4}$ & $A_{3}^{4} \oplus A_{1}^{6}$ \\
\hline$T^{4} / \mathbb{Z}_{6}$ & $A_{5} \oplus A_{2}^{4} \oplus A_{1}^{5}$ \\
\hline$T^{4} / \widehat{\mathcal{D}}_{4}$ & $D_{4}^{2} \oplus A_{3}^{3} \oplus A_{1}^{2}$ \\
\hline$T^{4} / \widehat{\mathcal{D}}_{4}$ & $D_{4}^{4} \oplus A_{1}^{3}$ \\
\hline$T^{4} / \widehat{\mathcal{D}}_{4}$ & $A_{3}^{6} \oplus A_{1}$ \\
\hline$T^{4} / \widehat{\mathcal{D}}_{5}$ & $D_{5} \oplus A_{3}^{3} \oplus A_{2}^{2} \oplus A_{1}$ \\
\hline$T^{4} / \mathbb{T}$ & $E_{6} \oplus D_{4} \oplus A_{2}^{4} \oplus A_{1}$ \\
\hline$T^{4} / \mathbb{T}$ & $A_{5} \oplus A_{3}^{2} \oplus A_{2}^{4}$ \\
\hline
\end{tabular}

Table 18: Singularities of torus quotients.

If we use two complex coordinates $\left(z_{1}, z_{2}\right)$ to describe the four-torus, the group $\mathbb{Z}_{m}$ is generated by

$$
\beta:\left(z_{1}, z_{2}\right) \rightarrow\left(\zeta z_{1}, \zeta^{-1} z_{2}\right)
$$

with $\zeta=e^{2 \pi i / m}$ an $m^{\text {th }}$ root of unity. The binary dihedral groups $\widehat{\mathcal{D}}_{m}$ have an $\mathbb{Z}_{2(m-2)}$ subgroup which acts just as $\mathbb{Z}_{m}$ described above, and in addition they have a generator $\alpha$ that we choose to act by

$$
\alpha:\left(z_{1}, z_{2}\right) \rightarrow\left(z_{2},-z_{1}\right) .
$$

The generators $\alpha$ and $\beta$ of $\widehat{\mathcal{D}}_{m}$ satisfy the relations

$$
\alpha^{2}=\beta^{m-2}, \quad \alpha^{4}=\beta^{2 m-4}=1, \quad \beta \alpha \beta=\alpha .
$$

Notice that we use the rank of the singularity to label the binary dihedral group $\widehat{\mathcal{D}}_{m}$; it is a group of dimension $4(m-2)$, and in the mathematics literature sometimes denoted by $D_{4(m-2)}^{*}$.

We first calculate the equivariant cohomology groups $H_{G}^{*}\left(T^{4}, U(1)\right)$ that will give us the flat equivariant $n$-forms. We can use the same spectral 
sequence described in section D.1, which is the Leray spectral sequence associated to the fibration $X \rightarrow X_{G} \rightarrow B G$. In the present case, the $E_{2}^{p, q}$ terms of this spectral sequence are given by

$$
E_{2}^{p, q}=H^{p}\left(G, H^{q}\left(T^{4}, U(1)\right)\right) .
$$

The right hand side is defined as follows. The action of $G$ on $T^{4}$ makes $H^{q}\left(T^{4}, U(1)\right)$ into a module for the finite group $G$. Then $E_{2}^{p, q}$ is defined as the cohomology of the finite group $G$ with values in this module.

Let us briefly review the standard definition of the cohomology of a finite group with values in a module. Given any $G$-module $M, n$-cochains with values in $M$ are maps

$$
c: G^{n} \rightarrow M,
$$

i.e., $M$-valued functions $c\left(g_{1}, \ldots, g_{n}\right)$ of $n$ group elements. On these cochains, we define a coboundary operator

$$
\begin{aligned}
\delta c\left(g_{1}, \ldots, g_{n+1}\right) & =c\left(g_{2}, \ldots, g_{n}\right)-(-1)^{n} g_{1} c\left(g_{2}, \ldots, g_{n+1}\right) \\
& +\sum_{i=1}^{n}(-1)^{i} c\left(g_{1}, \ldots, g_{i-1}, g_{i} g_{i+1}, g_{i+2}, \ldots, g_{n+1}\right) .
\end{aligned}
$$

The finite group cohomology $H^{*}(G, M)$ is given by $\operatorname{Ker}(\delta) / \operatorname{Im}(\delta)$.

Although this definition is quite simple, it is not very convenient for practical calculations. A more efficient way to compute finite group cohomology is to use a suitable resolution of the finite group $G$. More precisely, we need a right projective resolution of $\mathbb{Z}$ by $\mathbb{Z} G$-modules (see e.g. [106,107]). Such a resolution is an exact sequence

$$
\cdots \longrightarrow P_{k} \longrightarrow P_{k-1} \longrightarrow \cdots P_{0} \longrightarrow \mathbb{Z} \longrightarrow 0
$$

where all $P_{k}$ are projective modules over the group algebra $\mathbb{Z} G$, the maps are homomorphisms of $\mathbb{Z} G$-modules, and $\mathbb{Z}$ is viewed as the trivial $\mathbb{Z} G$-module. Projective modules are modules which are the direct summand of a free module. An exact sequence like (250) induces a sequence

$$
M^{G} \longrightarrow \operatorname{Hom}_{G}\left(P_{0}, M\right) \longrightarrow \operatorname{Hom}_{G}\left(P_{1}, M\right) \longrightarrow \operatorname{Hom}_{G}\left(P_{2}, M\right) \cdots
$$


for any $G$-module $M$. Here, $M^{G}$ denotes the $G$-invariant elements of $M$, and $\operatorname{Hom}_{G}(P, M)$ denotes the homomorphisms of $P$ to $M$ that commute with the action of $G$. It is a standard fact that (250) is no longer exact, but is still a complex. The cohomology of that complex is precisely the finite group cohomology $H^{*}(G, M)$.

A convenient resolution of finite groups is the Gruenberg resolution (see section 11.3 in [106]), which is associated to a presentation of the finite group (i.e., a set generators and relations). For $\mathbb{Z}_{m}$, we can use as generator $\beta$ subject to $\beta^{m}=1$, and for $\widehat{\mathcal{D}}_{k}$ we can use the presentation with generators $\alpha, \beta$ and relations (247). The respective resolutions are given on page 6 and 35 of [107]. Using (251) they yield a simple algorithm to compute the finite group cohomologies $H^{*}(G, M)$. Here we merely summarize the algorithm for $\mathbb{Z}_{m}$ and our particular $\widehat{\mathcal{D}}_{k}$ actions.

For $G=\mathbb{Z}_{m}$, the cohomology groups $H^{i}\left(\mathbb{Z}_{m}, M\right)$ are given by the cohomology of the complex

$$
M \stackrel{d_{0}}{\longrightarrow} M \stackrel{d_{1}}{\longrightarrow} M \stackrel{d_{0}}{\longrightarrow} M \stackrel{d_{1}}{\longrightarrow} \cdots
$$

where

$$
d_{0}(m)=(\beta-1) m, \quad d_{1}(m)=\sum_{g \in \mathbb{Z}_{m}} g m .
$$

Recall that $\beta$ was defined as the generator of $\mathbb{Z}_{m}$. In particular, we see that the cohomology in degrees above zero will be periodic with period 2. As an example, take $M=U(1)$ with the trivial action of $\mathbb{Z}_{m}$. Then $d_{0}=0$, $d_{1}=m$, and we get that $H^{0}\left(\mathbb{Z}_{m}, U(1)\right)=U(1), H^{2 i+1}\left(\mathbb{Z}_{m}, U(1)\right)=\mathbb{Z}_{m}$, and $H^{2 i}\left(\mathbb{Z}_{m}, U(1)\right)=0$ for $i>0$.

For $G=\widehat{\mathcal{D}}_{k}$, the cohomology groups are given by the cohomology of the complex

$$
M \stackrel{d_{0}}{\longrightarrow} M \oplus M \stackrel{d_{1}}{\longrightarrow} M \oplus M \stackrel{d_{2}}{\longrightarrow} M \stackrel{d_{3}}{\longrightarrow} M \stackrel{d_{0}}{\longrightarrow} M \oplus M \stackrel{d_{1}}{\longrightarrow} \cdots
$$


where

$$
\begin{aligned}
d_{0}(m)= & ((\alpha-1) m,(\beta-1) m), \\
d_{1}\left(m_{1}, m_{2}\right)= & \left((\beta-1) m_{1}+(\beta \alpha+1) m_{2},\right. \\
& \left.(-1-\alpha) m_{1}+\left(1+\beta+\ldots+\beta^{k-3}\right) m_{2}\right), \\
d_{2}\left(m_{1}, m_{2}\right)= & (1-\beta \alpha) m_{1}+(\beta-1) m_{2}, \\
d_{3}(m)= & \sum_{g \in \widehat{\mathcal{D}}_{k}} g m .
\end{aligned}
$$

Thus, this cohomology will be periodic with period four.

We are now ready to compute the $E_{2}^{p, q}$ terms defined in (248). First, we determine the $G$-module structure of $H^{q}\left(T^{4}, U(1)\right)$. We then insert this module in either (252) or (254), and work out the cohomology of the corresponding complex. In practice, the maps $d_{i}$ can always be written as matrices with integer coefficients with respect to an integral basis of $H^{q}\left(T^{4}, U(1)\right)$. Using suitable changes of basis by acting with $S L(p, \mathbb{Z})$, these matrices can be brought to a form with only diagonal elements. If the $k^{t h}$ differential $d_{k}$ has diagonal non-zero entries $d_{1}, \ldots, d_{r}$ then $H^{k}$ will be equal to $\mathbb{Z}_{d_{1}} \oplus \ldots \oplus \mathbb{Z}_{d_{r}}$.

The results of the calculation of $E_{2}^{p, q}$ are given below.

$G=\mathbb{Z}_{2}:$\begin{tabular}{|c|cccccc|}
\hline 4 & $U(1)$ & $\mathbb{Z}_{2}$ & 0 & $\mathbb{Z}_{2}$ & 0 & $\mathbb{Z}_{2}$ \\
3 & $\mathbb{Z}_{2}^{4}$ & 0 & $\mathbb{Z}_{2}^{4}$ & 0 & $\mathbb{Z}_{2}^{4}$ & 0 \\
2 & $U(1)^{6}$ & $\mathbb{Z}_{2}^{6}$ & 0 & $\mathbb{Z}_{2}^{6}$ & 0 & $\mathbb{Z}_{2}^{6}$ \\
1 & $\mathbb{Z}_{2}^{4}$ & 0 & $\mathbb{Z}_{2}^{4}$ & 0 & $\mathbb{Z}_{2}^{4}$ & 0 \\
0 & $U(1)$ & $\mathbb{Z}_{2}$ & 0 & $\mathbb{Z}_{2}$ & 0 & $\mathbb{Z}_{2}$ \\
\hline$q / p$ & 0 & 1 & 2 & 3 & 4 & 5 \\
\hline
\end{tabular}




$G=\mathbb{Z}_{3}:$\begin{tabular}{|c|cccccc|}
\hline 4 & $U(1)$ & $\mathbb{Z}_{3}$ & 0 & $\mathbb{Z}_{3}$ & 0 & $\mathbb{Z}_{3}$ \\
3 & $\mathbb{Z}_{3}^{2}$ & 0 & $\mathbb{Z}_{3}^{2}$ & 0 & $\mathbb{Z}_{3}^{2}$ & 0 \\
2 & $U(1)^{4}$ & $\mathbb{Z}_{3}^{3}$ & 0 & $\mathbb{Z}_{3}^{3}$ & 0 & $\mathbb{Z}_{3}^{3}$ \\
1 & $\mathbb{Z}_{3}^{2}$ & 0 & $\mathbb{Z}_{3}^{2}$ & 0 & $\mathbb{Z}_{3}^{2}$ & 0 \\
0 & $U(1)$ & $\mathbb{Z}_{3}$ & 0 & $\mathbb{Z}_{3}$ & 0 & $\mathbb{Z}_{3}$ \\
\hline$q / p$ & 0 & 1 & 2 & 3 & 4 & 5 \\
\hline
\end{tabular}

$G=\mathbb{Z}_{4}:$\begin{tabular}{|c|cccccc|}
\hline 4 & $U(1)$ & $\mathbb{Z}_{4}$ & 0 & $\mathbb{Z}_{4}$ & 0 & $\mathbb{Z}_{4}$ \\
3 & $\mathbb{Z}_{2}^{2}$ & 0 & $\mathbb{Z}_{2}^{2}$ & 0 & $\mathbb{Z}_{2}^{2}$ & 0 \\
2 & $U(1)^{4}$ & $\mathbb{Z}_{4}^{2} \oplus \mathbb{Z}_{2}^{2}$ & 0 & $\mathbb{Z}_{4}^{2} \oplus \mathbb{Z}_{2}^{2}$ & 0 & $\mathbb{Z}_{4}^{2} \oplus \mathbb{Z}_{2}^{2}$ \\
1 & $\mathbb{Z}_{2}^{2}$ & 0 & $\mathbb{Z}_{2}^{2}$ & 0 & $\mathbb{Z}_{2}^{2}$ & 0 \\
0 & $U(1)$ & $\mathbb{Z}_{4}$ & 0 & $\mathbb{Z}_{4}$ & 0 & $\mathbb{Z}_{4}$ \\
\hline$q / p$ & 0 & 1 & 2 & 3 & 4 & 5 \\
\hline
\end{tabular}

$G=\mathbb{Z}_{6}:$\begin{tabular}{|c|cccccc|}
\hline 4 & $U(1)$ & $\mathbb{Z}_{6}$ & 0 & $\mathbb{Z}_{6}$ & 0 & $\mathbb{Z}_{6}$ \\
3 & 0 & 0 & 0 & 0 & 0 & 0 \\
2 & $U(1)^{4}$ & $\mathbb{Z}_{6}^{3} \oplus \mathbb{Z}_{2}$ & 0 & $\mathbb{Z}_{6}^{3} \oplus \mathbb{Z}_{2}$ & 0 & $\mathbb{Z}_{6}^{3} \oplus \mathbb{Z}_{2}$ \\
1 & 0 & 0 & 0 & 0 & 0 & 0 \\
0 & $U(1)$ & $\mathbb{Z}_{6}$ & 0 & $\mathbb{Z}_{6}$ & 0 & $\mathbb{Z}_{6}$ \\
\hline$q / p$ & 0 & 1 & 2 & 3 & 4 & 5 \\
\hline
\end{tabular}

$$
G=\widehat{\mathcal{D}}_{4}: \begin{array}{|c|cccccc|}
\hline 4 & U(1) & \mathbb{Z}_{2}^{2} & 0 & \mathbb{Z}_{8} & 0 & \mathbb{Z}_{2}^{2} \\
3 & \mathbb{Z}_{2} & 0 & \mathbb{Z}_{2} & 0 & \mathbb{Z}_{2} & 0 \\
2 & U(1)^{3} & \mathbb{Z}_{4}^{3} & 0 & \mathbb{Z}_{4}^{3} & 0 & \mathbb{Z}_{4}^{3} \\
1 & \mathbb{Z}_{2} & 0 & \mathbb{Z}_{2} & 0 & \mathbb{Z}_{2} & 0 \\
0 & U(1) & \mathbb{Z}_{2}^{2} & 0 & \mathbb{Z}_{8} & 0 & \mathbb{Z}_{2}^{2} \\
\hline q / p & 0 & 1 & 2 & 3 & 4 & 5 \\
\hline
\end{array}
$$




$G=\widehat{\mathcal{D}}_{5}:$\begin{tabular}{|c|cccccc|}
\hline 4 & $U(1)$ & $\mathbb{Z}_{4}$ & 0 & $\mathbb{Z}_{12}$ & 0 & $\mathbb{Z}_{4}$ \\
3 & 0 & 0 & 0 & 0 & 0 & 0 \\
2 & $U(1)^{3}$ & $\mathbb{Z}_{4}^{2} \oplus \mathbb{Z}_{6}$ & 0 & $\mathbb{Z}_{4} \oplus \mathbb{Z}_{6} \oplus \mathbb{Z}_{12}$ & 0 & $\mathbb{Z}_{4}^{2} \oplus \mathbb{Z}_{6}$ \\
1 & 0 & 0 & 0 & 0 & 0 & 0 \\
0 & $U(1)$ & $\mathbb{Z}_{4}$ & 0 & $\mathbb{Z}_{12}$ & 0 & $\mathbb{Z}_{4}$ \\
\hline$q / p$ & 0 & 1 & 2 & 3 & 4 & 5 \\
\hline
\end{tabular}

For $G=\mathbb{Z}_{m}$, the rows in these tables are periodic with period two, whereas for $G=\widehat{\mathcal{D}}_{k}$ they are periodic with period four. In all cases, the spectral sequence collapses at the $E_{2}^{p, q}$ term. In addition, by redoing the calculation with coeffients in $\mathbb{Z}_{r}$ rather than $U(1)$, we find that the extension problem is trivial. Thus, the equivariant cohomologies $H_{G}^{n}\left(T^{4}, U(1)\right)$ are isomorphic to $\oplus_{i} E_{2}^{i, n-i}$.

As a simple test, we notice that all cohomology above degree four has to be supported purely by the singularities of $T^{4} / G$. This can be seen, for example, from a Mayer-Vietoris argument. Using Table 18 (see also Table 10), one readily verifies that this is indeed the case. Notice that $H^{4 i+1}\left(\widehat{\mathcal{D}}_{2 k}\right)=\mathbb{Z}_{2} \oplus \mathbb{Z}_{2}, H^{4 i+1}\left(\widehat{\mathcal{D}}_{2 k+1}\right)=\mathbb{Z}_{4}$, and $H^{4 i+3}\left(\widehat{\mathcal{D}}_{k}\right)=\mathbb{Z}_{4(k-2)}$.

\section{One-forms}

The equivariant 1-forms receive contributions from two different sources. One is from $H^{0}\left(G, H^{1}\left(T^{4}, U(1)\right)\right)$, which are the $G$-invariant 1-forms on $T^{4}$. They are in one-to-one correspondence with the $G$-fixed points on $T^{4}$. The second contribution is from

$$
H^{1}\left(G, H^{0}\left(T^{4}, U(1)\right)\right)=H^{1}(G, U(1)),
$$

whose elements correspond to the one-dimensional representations of $G$. Both have a clear interpretation in terms of line bundles over $T^{4}$ : the $G$ invariant 1-forms represent a flat connection on $T^{4}$ whereas $H^{1}(G, U(1))$ represents possible actions of $G$ on the fiber of the line bundle. Also note that $H_{G}^{1}\left(T^{4}, U(1)\right)$ matches with the group $\left(E^{\perp}\right)^{\perp} / E$ given in table 10. 
What is the $\mathrm{M}$ theory description of these theories? Let $A \cdot d x \in$ $H^{0}\left(G, H^{1}\left(T^{4}, U(1)\right)\right)$ denote an invariant 1-form, and $\rho \in H^{1}(G, U(1))$ a 1-dimensional representation of $G$. The pair $(A, \rho)$ labels a general element of $H_{G}^{1}\left(T^{4}, U(1)\right)$. The 1-form $A$ defines a five-torus $T^{5}$, which is not of the product form $T^{4} \times S^{1}$. Instead, the extra circle is fibered non-trivially over $T^{4}$. The five-torus has a metric of the form

$$
d s^{2}=d s_{T^{4}}^{2}+R_{5}^{2}(d \phi+A \cdot d x)^{2}
$$

where $\phi$ denotes the coordinate along the extra circle, whose radius we denoted by $R_{5}$. The group $G$ acts on this five-torus by

$$
(x, \phi) \rightarrow(g(x), \phi+\rho(g)+A \cdot(x-g(x))) .
$$

The $\mathrm{M}$ theory description of these theories is a compactification on $T^{5} / G$, with the five-torus and group action given in (262) and (263).

To relate this to compactifications on $\left(M \times S^{1}\right) / G$, we need to find a description where the five-torus is a direct product of a four-torus and a circle. As in section 4.4, this can be accomplished by taking a suitable cover of $T^{5}$. Suppose that the four-torus is described by $\mathbb{R}^{4} / \Lambda$. Then $A \in \mathbb{R}^{4} / \Lambda^{*}$ which is the dual four-torus. Let $p$ be the smallest positive integer such that $p A=0$ in $\mathbb{R}^{4} / \Lambda^{*}$. We define a $p$-fold cover $\hat{T}^{4}$ of $T^{4}$ as $\mathbb{R}^{4} / \Lambda_{A}$, where $\Lambda_{A}$ is the lattice

$$
\Lambda_{A}=\{x \in \Lambda \mid A \cdot x \in \mathbb{Z}\} .
$$

The group action on $T^{4}$ lifts to an action on $\hat{T}^{4}$. To prove this, we need to show that $g\left(\Lambda_{A}\right) \subset \Lambda_{A}$. Thus for $x \in \Lambda_{A}$, then we need to show that $g(x) \cdot A \in$ $\mathbb{Z}$. The statement that $A$ is $G$ invariant implies that $g(x) \cdot A-x \cdot A=x \cdot B$, with $B \in \Lambda^{*}$. Applying this to $x \in \Lambda_{A}$, we immediately get that $g(x) \cdot A \in \mathbb{Z}$. Therefore, $G$ lifts to an action on $\hat{T}^{4}$.

Clearly, $T^{4}=\hat{T}^{4} / G_{p}$, where $G_{p}$ is finite group of translations in $\hat{T}^{4}$. It is not difficult to see that $G_{p}=\mathbb{Z}_{p}$, because we can always use $S L(4, \mathbb{Z})$ to make only one of the components of $A$ non-zero. In particular, $\Lambda / \Lambda_{A}=\mathbb{Z}_{p}$, 
and we can choose an element $x_{0} \in \Lambda$ that generates $\Lambda / \Lambda_{A}$. Now on $\hat{T}^{4} \times S^{1}$, we have both an action of $\mathbb{Z}_{p}$ and of $G$. They act as

$$
G:(x, \phi) \rightarrow(g(x), \phi+\rho(g)), \quad \mathbb{Z}_{p}:(x, \phi) \rightarrow\left(x+x_{0}, \phi+A \cdot x_{0}\right),
$$

and one can easily verify that these two group actions commute. The $\mathrm{M}$ theory description of IIA string theory on $T^{4} / G$ with 1 -form flux $(A, \rho)$ is given by $\mathrm{M}$ theory on

$$
\left(T^{5}\right) / G \equiv\left(\hat{T}^{4} \times S^{1}\right) /\left(G \times \mathbb{Z}_{p}\right)
$$

We argue in section 4 that the only possible $M$ theory compactifications that preserve the right amount of supersymmetry are of the form $(M \times$ $\left.S^{1}\right) / \mathbb{Z}_{r}$, with $M=T^{4}$ or $M=K 3$. Indeed, the space (265) is always of this form. To find $M$, we need to study the action of $G \times \mathbb{Z}_{p}$ on the $S^{1}$ factor.

The image of $\rho$ in $U(1)$ defines a subgroup $\mathbb{Z}_{|\rho|}$ of $U(1)$. We also have a 1-dimensional representation of $G \times \mathbb{Z}_{p}$, acting on the $S^{1}$ factor in $\hat{T}^{4} \times S^{1}$. This representation has as image in $U(1)$ the group $\mathbb{Z}_{\mathrm{lcm}(p,|\rho|)}$, where $\mathrm{lcm}$ denotes the least common multiple. Thus there is an exact sequence

$$
\hat{G} \rightarrow G \times \mathbb{Z}_{p} \rightarrow \mathbb{Z}_{\operatorname{lcm}(p,|\rho|)},
$$

where $\hat{G}$ is a normal subgroup of $G$. Therefore, M theory compactified on (265) is the same as $\mathrm{M}$ theory compactified on

$$
\left(\left(\hat{T}^{4} / \hat{G}\right) \times S^{1}\right) / \mathbb{Z}_{\operatorname{lcm}(p,|\rho|)} .
$$

This is always of the form $\left(M \times S^{1}\right) / \mathbb{Z}_{r}$, with $M=T^{4}$ of $M=K 3$, as expected. We obtain $M=T^{4}$ only if $G$ is cyclic, $\rho$ is a faithful representation and $A=0$. In all other cases, $M=K 3$.

As an example, let us take our favorite non-abelian action $G=\widehat{\mathcal{D}}_{4}$. We showed that $E_{2}^{1,0}=\mathbb{Z}_{2}^{2}$ and that $E_{2}^{0,1}=\mathbb{Z}_{2}$. There is one case (the trivial 1-form) where $|\rho|=1$ and $p=1$. This corresponds to $\mathrm{M}$ theory on $\left(T^{4} / \widehat{\mathcal{D}}_{4}\right) \times S^{1}$, as expected. There are three cases with $|\rho|=2$ and $p=1$. 
These correspond to $\mathrm{M}$ theory on $\left(\left(\hat{T}^{4} / \mathbb{Z}_{4}\right) \times S^{1}\right) / \mathbb{Z}_{2}$. Furthermore, there is one case with $|\rho|=1$ and $p=2$. This corresponds to $\mathrm{M}$ theory on $\left(\left(\hat{T}^{4} / \widehat{\mathcal{D}}_{4}\right) \times S^{1}\right) / \mathbb{Z}_{2}$. Finally, there are three cases with $|\rho|=2$ and $p=1$. These correspond to $\mathrm{M}$ theory on $\left(\left(\hat{T}^{4} / \mathbb{Z}_{4}\right) \times S^{1}\right) / \mathbb{Z}_{2}$.

What about the holonomies of these 1-forms? Consider a point $p \in \mathbb{R}^{4}$ that is fixed under a part $G_{p}$ of the group $G$ when viewed as an element of $\mathbb{R}^{4} / \Lambda$. Given a group element $g$ that fixes $p$, we need to determine the holonomy of the 1-form around the loop associated to $g$ in $S^{3} / G_{p}$. Here, we view $g$ as an element of $\pi_{1}\left(S^{3} / G_{p}\right) \cong G_{p}$. Generalizing the discussion at the end of section 4.4 , we find for the holonomy

$$
\exp [2 \pi i((p-g(p)) \cdot A+\rho(g))]
$$

\section{D.3 Explicit generators for $H_{\mathbb{Z}_{2}}^{2}\left(T^{n}, \mathbb{Z}_{2}\right)$}

To conclude our discussion of equivariant cohomology, we shall explicitly construct generators for $H_{\mathbb{Z}_{2}}^{2}\left(T^{n}, \mathbb{Z}_{2}\right)$ where $n=1,2,3$. This makes the localization of the $B$-field concrete in the orientifold constructions discussed in section 3. We use the cell decomposition of the space $T_{\mathbb{Z}_{2}}^{n}=S^{N} \times_{\mathbb{Z}_{2}} T^{n}$ which is obtained from a $\mathbb{Z}_{2}$-equivariant cell-decomposition of $S^{N} \times T^{n}$. We are interested in the second cohomology so it is sufficient to take $N=3$ here. Throughout our discussion, we will use the standard cell decomposition of $S^{N}$

$$
S^{N}=e_{0}^{+} \cup e_{0}^{-} \cup e_{1}^{+} \cup e_{1}^{-} \cup e_{2}^{+} \cup e_{2}^{-} \cup \cdots
$$

where the $\mathbb{Z}_{2}$ exchanges $e_{m}^{+}$and $e_{m}^{-}$,

$$
e_{m}^{ \pm} \rightarrow e_{m}^{\mp}
$$




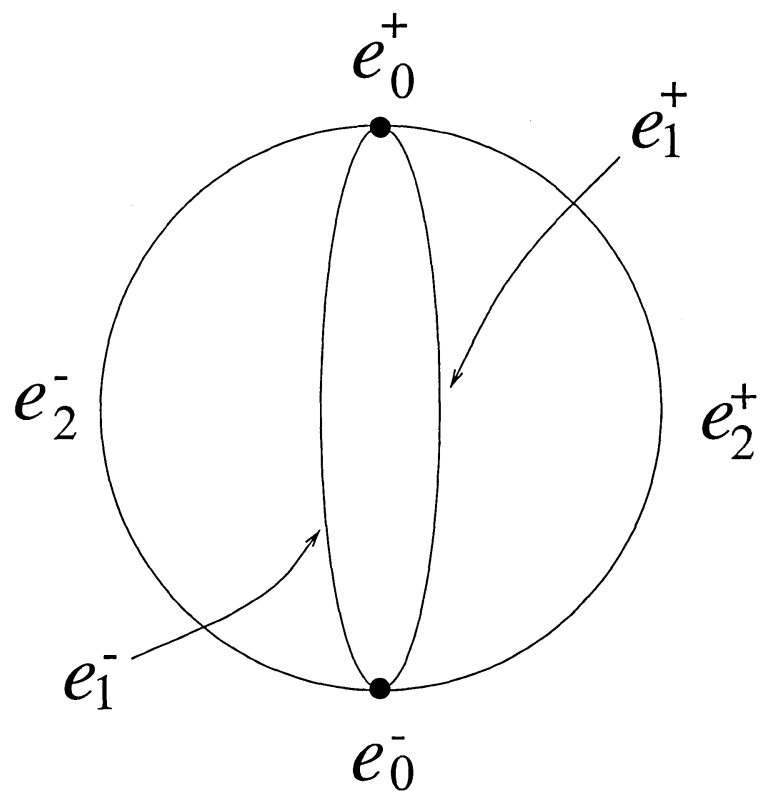

Figure 5: Cell decomposition of $S^{2}$.

This decomposition is illustrated in figure 5. The boundary operator is given by

$$
\begin{aligned}
& \partial e_{0}^{ \pm}=0, \\
& \partial e_{1}^{ \pm}=e_{0}^{+}+e_{0}^{-}, \\
& \partial e_{2}^{ \pm}=e_{1}^{+}+e_{1}^{-},
\end{aligned}
$$

We note that we do not care about the sign since we are considering the $\mathbb{Z}_{2}$ coefficient, $e_{m}^{ \pm}=-e_{m}^{ \pm}$.

$\underline{n=1}$

We consider the cell decomposition of $T^{1}$ as depicted in figure 6 . The $\mathbb{Z}_{2}$ inversion fixes the 0-chains but exchanges the two 1-chains,

$$
e_{0}^{ \pm} \rightarrow e_{0}^{ \pm}, \quad e_{1}^{ \pm} \rightarrow e_{1}^{\mp} .
$$

The boundary operator is just $\partial e_{0}^{ \pm}=0$ and $\partial e_{1}^{ \pm}=e_{0}^{+}+e_{0}^{-}$. 


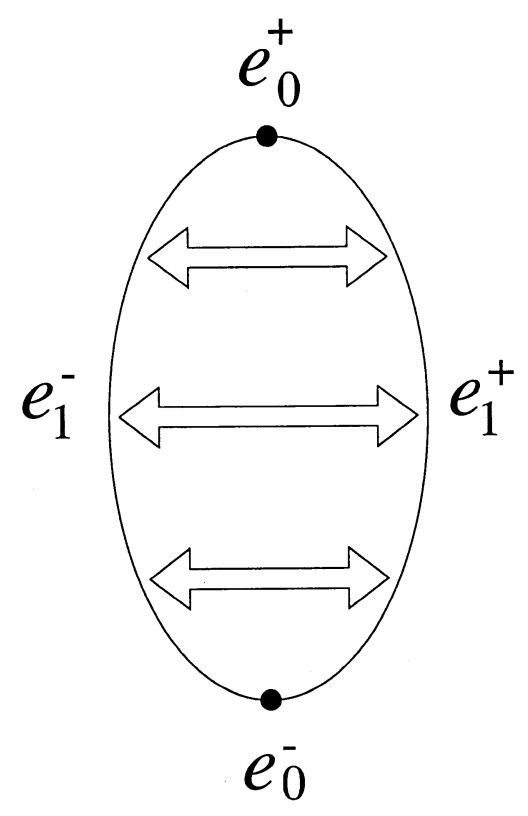

Figure 6: Cell decomposition of $T^{1}$.

The cell decomposition of the space $T_{\mathbb{Z}_{2}}^{1}=\left(S^{N} \times T^{1}\right) / \mathbb{Z}_{2}$ is given as follows:

$$
\left.\begin{array}{l}
e_{00}^{ \pm}:=e_{0}^{+} \times e_{0}^{ \pm} \equiv e_{0}^{-} \times e_{0}^{ \pm}, \\
e_{\ell 0}^{ \pm}:=e_{\ell}^{+} \times e_{0}^{ \pm} \equiv e_{\ell}^{-} \times e_{0}^{ \pm}, \\
e_{(\ell-1) 1}^{ \pm}:=e_{\ell-1}^{+} \times e_{1}^{ \pm} \equiv e_{\ell-1}^{-} \times e_{1}^{\mp},
\end{array}\right\} \quad \ell=1,2,3, \ldots
$$

It is then easy to see that the boundary operator is given by,

$$
\begin{aligned}
& \partial e_{\ell 0}^{ \pm}=0, \quad \ell=0,1,2, \ldots \\
& \partial e_{01}^{ \pm}=e_{00}^{+}+e_{00}^{-}, \\
& \partial e_{\ell 1}^{ \pm}=e_{(\ell-1) 1}^{+}+e_{(\ell-1) 1}^{-}+e_{\ell 0}^{+}+e_{\ell 0}^{-}, \quad \ell=1,2,3, \ldots
\end{aligned}
$$

By dualization we find the following coboundary operator, where the notation for the cochain should be self-evident:

$$
\delta c_{\ell \alpha}^{ \pm}=c_{(\ell+\alpha) 1}^{+}+c_{(\ell+\alpha) 1}^{-}, \quad(\ell=0,1,2, \ldots ; \quad \alpha=0,1) .
$$

From this expression, we see that $B^{2}$ (the space of coboundaries of dimension 2 ) is spanned by $\left(c_{11}^{+}+c_{11}^{-}\right)$while $Z^{2}$ (the space of 2-cocycles) is spanned by 
$\left(c_{20}^{+}+c_{11}^{+}\right),\left(c_{20}^{-}+c_{11}^{+}\right)$, and $\left(c_{11}^{+}+c_{11}^{-}\right)$. The cohomology group $H^{2}=Z^{2} / B^{2}$ is therefore given by

$$
\mathbb{Z}_{2}\left[c_{20}^{+}+c_{11}^{+}\right] \oplus \mathbb{Z}_{2}\left[c_{20}^{-}+c_{11}^{+}\right]
$$

The first generator has value 1 on $e_{20}^{+}$i.e., $\mathbb{R P}^{2}$ at the $\mathbb{Z}_{2}$ fixed point $e_{0}^{+}$, while it vanishes on $e_{20}^{-}$, i.e., $\mathbb{R P}^{2}$ at the other $\mathbb{Z}_{2}$ fixed point $e_{0}^{-}$. The second generator vanishes on $e_{20}^{+}\left(\mathbb{R P}^{2}\right.$ at $\left.e_{0}^{+}\right)$while it has value 1 on $e_{20}^{-}\left(\mathbb{R P}^{2}\right.$ at $\left.e_{0}^{-}\right)$.

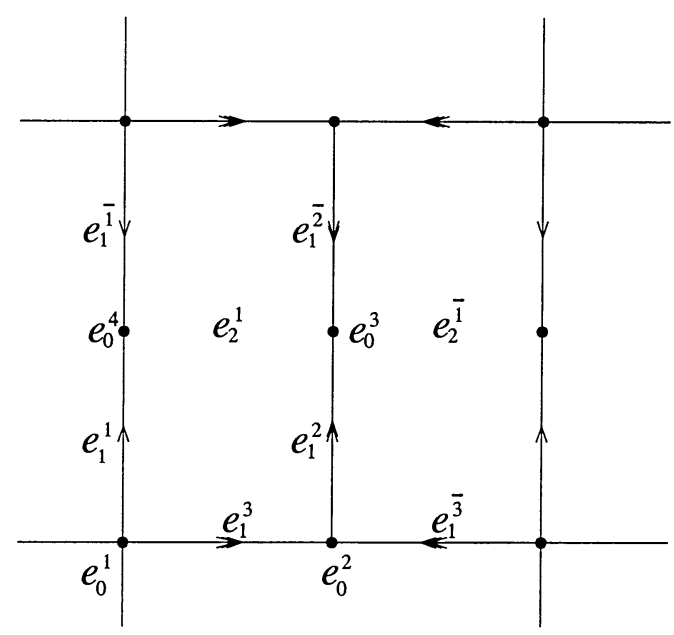

Figure 7: Cell decomposition of $T^{2}$.

$\underline{n=2}$

We consider the $\mathbb{Z}_{2}$-equivariant cell decomposition of $T^{2}$ depicted in figure 7. There are four 0 -chains $e_{0}^{i}$ where $i=1,2,3,4$. In addition, there are six 1 -chains $e_{1}^{\mu}$ with $\mu=1, \overline{1}, 2, \overline{2}, 3, \overline{3}$ and two 2 -chains $e_{2}^{I}$ with $I=1, \overline{1}$. The $\mathbb{Z}_{2}$ acts on them in the following way:

$$
e_{0}^{i} \rightarrow e_{0}^{i}, \quad e_{1}^{\mu} \rightarrow e_{1}^{\bar{\mu}}, \quad e_{2}^{I} \rightarrow e_{2}^{\bar{I}} .
$$


In this expression, $\overline{\overline{2}}=2$ etc. The cell decomposition of $T_{\mathbb{Z}_{2}}^{2}$ is given by

$$
\begin{aligned}
& e_{00}^{i}:=e_{0}^{+} \times e_{0}^{i} \equiv e_{0}^{-} \times e_{0}^{i}, \\
& e_{10}^{i}:=e_{1}^{+} \times e_{0}^{i} \equiv e_{1}^{-} \times e_{0}^{i}, \\
& e_{01}^{\mu}:=e_{0}^{+} \times e_{1}^{\mu} \equiv e_{0}^{-} \times e_{1}^{\bar{\mu}}, \\
& \left.\begin{array}{l}
e_{\ell 0}^{i}:=e_{\ell}^{+} \times e_{0}^{i} \equiv e_{\ell}^{-} \times e_{0}^{i}, \\
e_{(\ell-1) 1}^{\mu}:=e_{\ell-1}^{+} \times e_{1}^{\mu} \equiv e_{\ell-1}^{-} \times e_{1}^{\bar{\mu}}, \\
e_{(\ell-2) 2}^{I}:=e_{\ell-2}^{+} \times e_{2}^{I} \equiv e_{\ell-2}^{-} \times e_{2}^{\bar{I}}
\end{array}\right\} \quad \ell=2,3, \ldots
\end{aligned}
$$

Here, we do not show all the details of the computation but just present the result. The $2^{\text {nd }}$ coboundary group $B^{2}$ is spanned by $c_{02}^{1}+c_{02}^{\overline{1}}$ and $c_{11}^{\mu}+c_{11}^{\bar{\mu}}$ with $\mu=1,2,3$. The cohomology is of rank $4, H_{\mathbb{Z}_{2}}^{2}\left(T^{2}, \mathbb{Z}_{2}\right)=\left(\mathbb{Z}_{2}\right)^{4}$, and the four generators are represented by

$$
\begin{array}{cc}
c_{20}^{1}+c_{11}^{1}+c_{11}^{3}+c_{02}^{1}, & c_{20}^{2}+c_{11}^{2}+c_{11}^{3}+c_{02}^{1} \\
c_{20}^{3}+c_{11}^{2}+c_{02}^{1}, & c_{20}^{4}+c_{11}^{1}+c_{02}^{1} .
\end{array}
$$

Note that the $i^{\text {th }}$ generator has a non-trivial value on the $\mathbb{R P}^{2}$ at the fixed point $e_{0}^{i}$ (i.e., on the cycle $e_{20}^{i}$ ), and is vanishing at the other fixed points $e_{0}^{j}$, $j \neq i$.

$\underline{n=3}$

We consider the $\mathbb{Z}_{2}$-equivariant cell decomposition of $T^{3}$ depicted in figure 8. There are eight 0 -chains $e_{0}^{i}(i=A, B, C, \ldots, H)$; fourteen 1-chains $e_{1}^{\mu}(\mu=a, \bar{a}, b, \bar{b}, \ldots, g, \bar{g})$; eight 2-chains $e_{2}^{I}(I=\alpha, \bar{\alpha}, \ldots, \delta, \bar{\delta})$; and two 3 -chains $e_{3}^{\lambda}(\lambda=1, \overline{1})$. The $\mathbb{Z}_{2}$ acts on them as follows,

$$
e_{0}^{i} \rightarrow e_{0}^{i}, \quad e_{1}^{\mu} \rightarrow e_{1}^{\bar{\mu}}, \quad e_{2}^{I} \rightarrow e_{2}^{\bar{I}}, \quad e_{3}^{\lambda} \rightarrow e_{3}^{\bar{\lambda}} .
$$




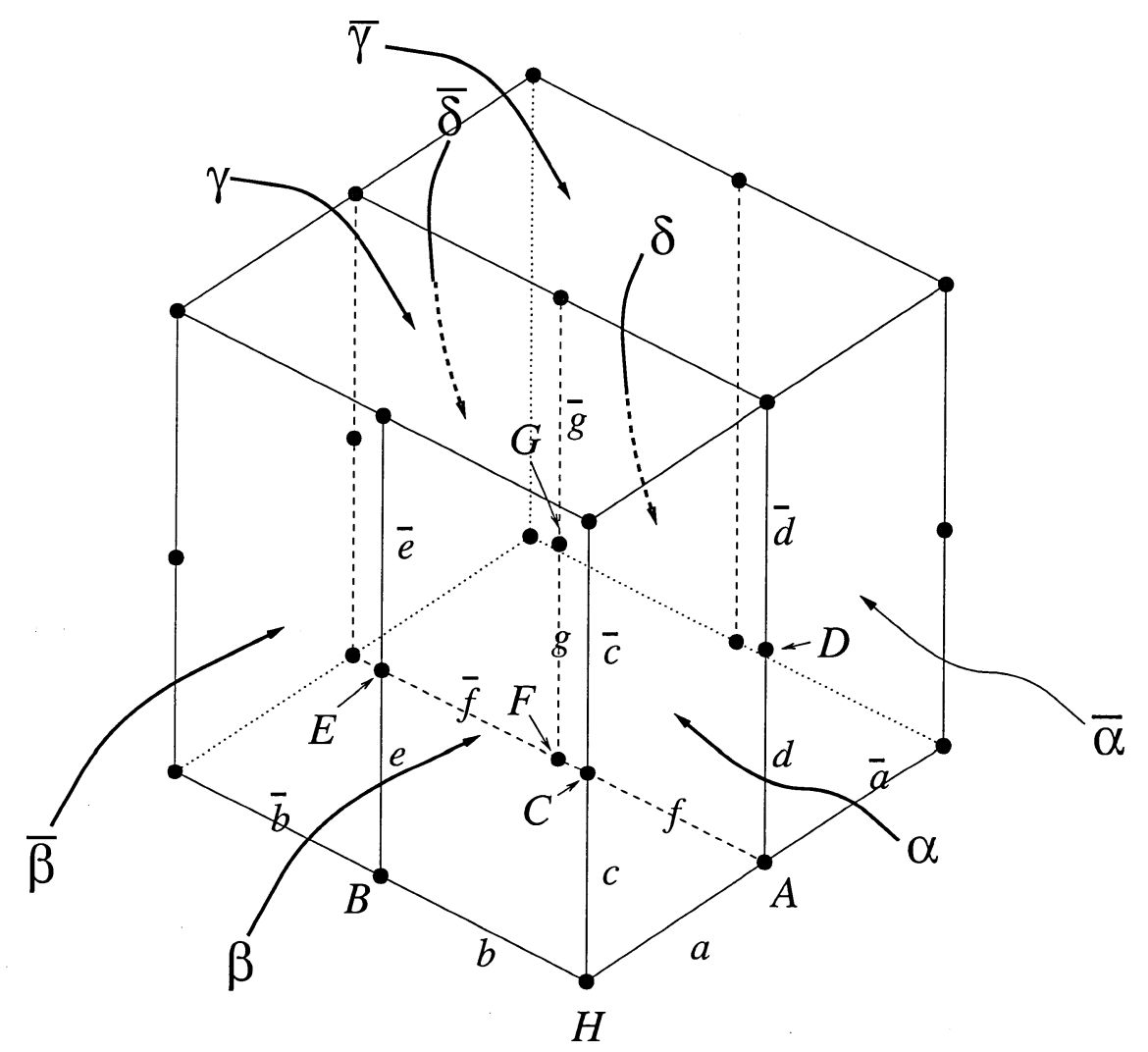

Figure 8: Cell decomposition of $T^{3}$.

The cell decomposition of $T_{\mathbb{Z}_{2}}^{3}$ is given by

$$
\left.\begin{array}{l}
e_{00}^{i}:=e_{0}^{+} \times e_{0}^{i} \equiv e_{0}^{-} \times e_{0}^{i}, \\
e_{10}^{i}:=e_{1}^{+} \times e_{0}^{i} \equiv e_{1}^{-} \times e_{0}^{i}, \\
e_{01}^{\mu}:=e_{0}^{+} \times e_{1}^{\mu} \equiv e_{0}^{-} \times e_{1}^{\mu},
\end{array}\right\}
$$

As in the previous case, we only present the result. The $2^{\text {nd }}$ coboundary group $B^{2}$ is spanned by $c_{02}^{I}+c_{02}^{\bar{I}}$ for all $I$ and $c_{11}^{\mu}+c_{11}^{\bar{\mu}}$ for all $\mu$. The 
cohomology is of rank $7, H_{\mathbb{Z}_{2}}^{2}\left(T^{3}, \mathbb{Z}_{2}\right)=\left(\mathbb{Z}_{2}\right)^{7}$, and the seven generators are represented by

$$
\begin{aligned}
& c_{20}^{A}+c_{20}^{H}+c_{11}^{b}+c_{11}^{c}+c_{11}^{d}+c_{11}^{f}+c_{02}^{\beta}+c_{02}^{\delta}, \\
& c_{20}^{B}+c_{20}^{H}+c_{11}^{a}+c_{11}^{c}+c_{11}^{d}+c_{02}^{\beta}+c_{02}^{\delta}, \\
& c_{20}^{C}+c_{20}^{H}+c_{11}^{a}+c_{11}^{b}+c_{02}^{\gamma}, \\
& c_{20}^{D}+c_{20}^{H}+c_{11}^{a}+c_{11}^{b}+c_{11}^{c}+c_{11}^{d}+c_{02}^{\beta}+c_{02}^{\gamma}+c_{02}^{\delta}, \\
& c_{20}^{E}+c_{20}^{H}+c_{11}^{a}+c_{11}^{b}+c_{11}^{c}+c_{11}^{e}+c_{02}^{\alpha}+c_{02}^{\gamma}, \\
& c_{20}^{F}+c_{20}^{H}+c_{11}^{a}+c_{11}^{b}+c_{11}^{c}+c_{11}^{f}+c_{11}^{g}+c_{02}^{\alpha}+c_{02}^{\beta}+c_{02}^{\delta}, \\
& c_{20}^{G}+c_{20}^{H}+c_{11}^{a}+c_{11}^{b}+c_{11}^{c}+c_{11}^{g}+c_{02}^{\alpha}+c_{02}^{\beta}+c_{02}^{\gamma}+c_{02}^{\delta} .
\end{aligned}
$$

Note that the $i^{\text {th }}$ generator has a non-trivial value on the $\mathbb{R P}^{2}$ at the fixed point $e_{0}^{i}$ (i.e., on the cycle $e_{20}^{i}$ ) and also at the point $e_{0}^{H}$ (i.e., on the cycle $\left.e_{20}^{H}\right)$, and is vanishing at the other fixed points $e_{0}^{j}, j \neq i, H$.

\section{References}

[1] S. Chaudhuri, G. Hockney and J. D. Lykken, "Maximally supersymmetric string theories in D < 10," Phys. Rev. Lett. 75, 2264 (1995) [hep-th/9505054].

[2] S. Chaudhuri and J. Polchinski, "Moduli space of CHL strings," Phys. Rev. D52 (1995) 7168 [hep-th/9506048].

[3] M. Fabinger and P. Horava, "Casimir effect between world-branes in heterotic M-theory," Nucl. Phys. B 580, 243 (2000) [hep-th/0002073].

[4] D. Diaconescu, G. Moore and E. Witten, " $\mathrm{E}(8)$ gauge theory, and a derivation of K-theory from M-theory," hep-th/0005090.

[5] O. Bergman, E. Gimon and S. Sugimoto, "Orientifolds, RR torsion, and K-theory," hep-th/0103183. 
[6] Y. Hyakutake, Y. Imamura and S. Sugimoto, "Orientifold planes, type I Wilson lines and non-BPS D-branes," JHEP 0008, 043 (2000) [hepth/0007012].

[7] O. Bergman and A. Fayyazuddin, "String junctions and BPS states in Seiberg-Witten theory," Nucl. Phys. B 531, 108 (1998) [hepth/9802033].

[8] A. Mikhailov, N. Nekrasov and S. Sethi, "Geometric realizations of BPS states in N=2 theories," Nucl. Phys. B 531, 345 (1998) [hepth/9803142].

[9] O. DeWolfe, T. Hauer, A. Iqbal and B. Zwiebach, "Constraints on the BPS spectrum of $\mathrm{N}=2, \mathrm{D}=4$ theories with A-D-E flavor symmetry," Nucl. Phys. B 534, 261 (1998) [hep-th/9805220].

[10] Y. Imamura, "String junctions and their duals in heterotic string theory," Prog. Theor. Phys. 101, 1155 (1999) [hep-th/9901001].

[11] M. Fukae, Y. Yamada and S. Yang, "Mordell-Weil lattice via string junctions," Nucl. Phys. B 572, 71 (2000) [hep-th/9909122].

[12] J. Hashiba, K. Hosomichi and S. Terashima, "String junctions in B field background," JHEP0009, 008 (2000) [hep-th/0005164].

[13] D. R. Morrison, "Wilson Lines in F-Theory," Lecture at Harvard University, 8 January 1999 (unpublished).

[14] J. H. Schwarz and A. Sen, "Type IIA dual of the six-dimensional CHL compactification," Phys. Lett. B357, 323 (1995) [hep-th/9507027].

[15] E. Witten, "New 'gauge' theories in six dimensions," JHEP 9801 (1998) 001 [hep-th/9710065].

[16] K. Landsteiner and E. Lopez, "New curves from branes," Nucl. Phys. B516, 273 (1998) [hep-th/9708118]. 
[17] E. Witten, "Toroidal compactification without vector structure," JHEP 9802, 006 (1998) [hep-th/9712028].

[18] D. S. Freed and E. Witten, "Anomalies in string theory with D-branes," hep-th/9907189.

[19] J. A. Keurentjes, Ph.D. thesis, "New vacua for Yang-Mills theory on a 3-torus," hep-th/0007196.

[20] A. Keurentjes, A. Rosly and A. V. Smilga, "Isolated vacua in supersymmetric Yang-Mills theories," Phys. Rev. D58, 081701 (1998) [hepth/9805183].

[21] A. Keurentjes, "Non-trivial flat connections on the 3-torus. I: G(2) and the orthogonal groups," JHEP 9905, 001 (1999) [hep-th/9901154].

[22] V. G. Kac and A. V. Smilga, "Vacuum structure in supersymmetric Yang-Mills theories with any gauge group," hep-th/9902029.

[23] A. Keurentjes, "Non-trivial flat connections on the 3-torus. II: The exceptional groups $\mathrm{F}(4)$ and $\mathrm{E}(6,7,8)$," JHEP 9905, 014 (1999) [hepth/9902186].

[24] A. Borel, R. Friedman and J. W. Morgan, "Almost commuting elements in compact Lie groups," math.GR/9907007.

[25] E. Witten, "Supersymmetric index in four-dimensional gauge theories," hep-th/0006010.

[26] G. 't Hooft, "A Property Of Electric And Magnetic Flux In Nonabelian Gauge Theories," Nucl. Phys. B153 (1979) 141.

[27] C. Schweigert, "On moduli spaces of flat connections with non-simply connected structure group," Nucl. Phys. B492, 743 (1997) [hepth/9611092].

[28] M. Berkooz, R. G. Leigh, J. Polchinski, J. H. Schwarz, N. Seiberg and E. Witten, "Anomalies, Dualities, and Topology of $\mathrm{D}=6 \mathrm{~N}=1$ Superstring Vacua," Nucl. Phys. B475, 115 (1996) [hep-th/9605184]. 
[29] A. Keurentjes, "Orientifolds and twisted boundary conditions," Nucl. Phys. B 589 (2000) 440 [hep-th/0004073].

[30] P. Hořava and E. Witten, "Heterotic and type I string dynamics from eleven dimensions," Nucl. Phys. B460, 506 (1996) [hep-th/9510209].

[31] E. Witten, "On flux quantization in M-theory and the effective action," J. Geom. Phys. 22, 1 (1997) [hep-th/9609122].

[32] K. S. Narain, "New Heterotic String Theories In Uncompactified Dimensions < 10," Phys. Lett. B169 (1986) 41.

[33] K. S. Narain, M. H. Sarmadi and E. Witten, "A Note On Toroidal Compactification Of Heterotic String Theory," Nucl. Phys. B279 (1987) 369 .

[34] K. S. Narain, M. H. Sarmadi and C. Vafa, "Asymmetric Orbifolds," Nucl. Phys. B288 (1987) 551.

[35] K. S. Narain, M. H. Sarmadi and C. Vafa, "Asymmetric orbifolds: Path integral and operator formulations," Nucl. Phys. B356 (1991) 163.

[36] W. Lerche, C. Schweigert, R. Minasian and S. Theisen, "A note on the geometry of CHL heterotic strings," Phys. Lett. B424 (1998) 53 [hep-th/9711104].

[37] V. V Nikulin, "Finite automorphism groups of Kähler K3 surfaces," Trans. Moscow. Math. Soc. 38 (1979) 71.

[38] A. Mikhailov, "Momentum lattice for CHL string," Nucl. Phys. B534 (1998) 612 [hep-th/9806030].

[39] P. Ginsparg, "Comment On Toroidal Compactification Of Heterotic Superstrings," Phys. Rev. D35, 648 (1987).

[40] P. Goddard, J. Nuyts and D. Olive, "Gauge Theories And Magnetic Charge," Nucl. Phys. B125 (1977) 1. 
[41] S. Chaudhuri and D. A. Lowe, "Type IIA heterotic duals with maximal supersymmetry," Nucl. Phys. B459, 113 (1996) [hep-th/9508144].

[42] P. S. Aspinwall, "Some relationships between dualities in string theory," Nucl. Phys. Proc. Suppl. 46, 30 (1996) [hep-th/9508154].

[43] M. Bianchi, G. Pradisi and A. Sagnotti, "Toroidal compactification and symmetry breaking in open string theories," Nucl. Phys. B376, 365 (1992).

[44] A. Sen and S. Sethi, "The mirror transform of type I vacua in six dimensions," Nucl. Phys. B499, 45 (1997) [hep-th/9703157].

[45] E. Witten, "Baryons and branes in anti de Sitter space," JHEP 9807 (1998) 006 [hep-th/9805112].

[46] K. Hori, "Consistency condition for fivebrane in M-theory on $R^{5} / Z_{2}$ orbifold," Nucl. Phys. B539 (1999) 35 [hep-th/9805141].

[47] E. G. Gimon, "On the M-theory interpretation of orientifold planes," [hep-th/9806226].

[48] S. Sethi, "A relation between $N=8$ gauge theories in three dimensions," JHEP 9811 (1998) 003 [hep-th/9809162].

[49] M. Berkooz and A. Kapustin, "New IR dualities in supersymmetric gauge theory in three dimensions," JHEP9902, 009 (1999) [hepth/9810257].

[50] A. Hanany, B. Kol and A. Rajaraman, "Orientifold points in M theory," JHEP 9910 (1999) 027 [hep-th/9909028].

[51] H. Kao and K. Lee, "Selfdual Chern-Simons systems with an N=3 extended supersymmetry," Phys. Rev. D46, 4691 (1992) [hep$\operatorname{th} / 9205115]$.

[52] A. Dabholkar and J. Park, "Strings on Orientifolds," Nucl. Phys. B477, 701 (1996) [hep-th/9604178]. 
[53] J. Park, "Orientifold and F-theory duals of CHL strings," Phys. Lett. B418, 91 (1998) [hep-th/9611119].

[54] A. Sen, "F-theory and Orientifolds," Nucl. Phys. B475, 562 (1996) [hep-th/9605150].

[55] A. L. Besse, Einstein manifolds, Springer-Verlag, Berlin, 1987.

[56] A. Fujiki, "Finite automorphism groups of complex tori of dimension two," Publ. Res. Inst. Math. Sci. 24 (1988), no. 1, 1.

[57] V. V Nikulin, "Integer symmetric bilinear forms and some of their geometric applications," Math. USSR Izvestija 14 (1980) 103.

[58] F. Enriques and F. Severi, "Mémoire sur les surfaces hyperelliptiques," Acta Math. 32 (1909), 283-392; 33 (1910), 321-403.

[59] V. V. Nikulin, "Kummer surfaces," Izv. Akad. Nauk SSSR Ser. Mat. 39 (1975), no. 2, 278-293, 471.

[60] D. R. Morrison, "On K3 surfaces with large Picard number," Invent. Math. 75 (1984), 105.

[61] J. Bertin, "Réseaux de Kummer et surfaces K3," Invent. Math. 93 (1988), 267.

[62] K. Wendland, "Consistency of orbifold conformal field theories on K3," hep-th/0010281.

[63] B. R. Greene, A. Shapere, C. Vafa and S. Yau, "Stringy Cosmic Strings And Noncompact Calabi-Yau Manifolds," Nucl. Phys. B 337, 1 (1990).

[64] M. Gross and P. M. H. Wilson, "Large Complex Structure Limits of K3 Surfaces," math.DG/0008018.

[65] J. H. Schwarz, "An SL(2,Z) multiplet of type IIB superstrings," Phys. Lett. B 360, 13 (1995) [hep-th/9508143]. 
[66] C. Vafa, "Evidence for F-Theory," Nucl. Phys. B469, 403 (1996) [hepth/9602022].

[67] P. Berglund, J. Ellis, A. E. Faraggi, D. V. Nanopoulos and Z. Qiu, "Elevating the free-fermion $\mathrm{Z}(2) \times \mathrm{Z}(2)$ orbifold model to a compactification of F-theory," Int. J. Mod. Phys. A 15, 1345 (2000) [hep-th/9812141].

[68] D. A. Cox, "Mordell-Weil groups of elliptic curves over $\mathbb{C}(t)$ with $p_{g}=0$ or 1," Duke Math. J. 49 (1982) 677.

[69] S. Katz and D. R. Morrison, "Gorenstein threefold singularities with small resolutions via invariant theory for Weyl groups," J. Algebraic Geom. 1 (1992), 449 [alg-geom/9202002].

[70] D. R. Morrison and N. Seiberg, "Extremal transitions and fivedimensional supersymmetric field theories," Nucl. Phys. B 483, 229 (1997) [hep-th/9609070].

[71] F. A. Cachazo and C. Vafa, "Type I' and real algebraic geometry," hep-th/0001029.

[72] R. Friedman, J. Morgan and E. Witten, "Vector bundles and F theory," Commun. Math. Phys. 187 (1997) 679 [hep-th/9701162].

[73] P. B. Kronheimer, "Instanton invariants and flat connections on the Kummer surface," Duke Math. J. 64 (1991), no. 2, 229-241.

[74] T. Bridgeland, A. King and M. Reid, "Mukai implies McKay: the McKay correspondence as an equivalence of derived categories," math.AG/9908027.

[75] E. Witten, "D-branes and K-theory," JHEP9812, 019 (1998) [hepth/9810188].

[76] R. Minasian and G. Moore, "K-theory and Ramond-Ramond charge," JHEP9711, 002 (1997) [hep-th/9710230]. 
[77] G. Moore and E. Witten, "Self-duality, Ramond-Ramond fields, and K-theory," JHEP0005, 032 (2000) [hep-th/9912279].

[78] D. S. Freed and M. J. Hopkins, "On Ramond-Ramond fields and Ktheory," JHEP0005 (2000) 044 [hep-th/0002027].

[79] D. S. Freed, "Dirac charge quantization and generalized differential cohomology," hep-th/0011220.

[80] J. Lott, "R/ZZ-Index Theory," Comm. in Anal. and Geom. 2 (1994) 279.

[81] M. F. Atiyah, V. Patodi, and I. M. Singer, "Spectral Asymmetry and Riemannian Geometry," Math. Proc. Camb. Phil. Soc. 77 (1975) 43.

[82] H. Garcia-Compean, "D-branes in orbifold singularities and equivariant K-theory," Nucl. Phys. B 557, 480 (1999) [hep-th/9812226].

[83] D. Diaconescu and J. Gomis, "Fractional branes and boundary states in orbifold theories," JHEP0010, 001 (2000) [hep-th/9906242].

[84] O. Bergman, E. Gimon, and B. Kol, "Strings on Orbifold Lines," hepth/0102095.

[85] R. Dijkgraaf and E. Witten, "Topological Gauge Theories And Group Cohomology," Commun. Math. Phys. 129, 393 (1990).

[86] M. de Wild Propitius, Topological interactions in broken gauge theories, Ph.D. thesis, University of Amsterdam, 1995, hep-th/9511195.

[87] M. F. Atiyah and G. B. Segal, "Equivariant K-Theory and Completion," J. Diff. Geom. 3 (1968).

[88] E. R. Sharpe, "Analogues of discrete torsion for the M-theory threeform," hep-th/0008170.

[89] G. Moore, "Some comments on branes, G-flux, and K-theory," hepth/0012007. 
[90] N. Seiberg, "IR dynamics on branes and space-time geometry," Phys. Lett. B384, 81 (1996) [hep-th/9606017].

[91] N. Seiberg and E. Witten, "Gauge dynamics and compactification to three dimensions," hep-th/9607163.

[92] A. Sen, "A note on enhanced gauge symmetries in M- and string theory," JHEP9709, 001 (1997) [hep-th/9707123].

[93] R. Miranda and D. R. Morrison, "The number of embeddings of integral quadratic forms. I, II," Proc. Japan Academy, Ser. A, 61, (1985) 317; 62, (1986) 29.

[94] A. Kapustin, "D-branes in a topologically nontrivial B-field," Adv. Theor. Math. Phys. 4, 127 (2001) [hep-th/9909089].

[95] P. S. Aspinwall, "Enhanced gauge symmetries and K3 surfaces," Phys. Lett. B357, 329 (1995) [hep-th/9507012].

[96] E. Gava, A. B. Hammou, J. F. Morales and K. S. Narain, "D1/D5 systems in $N=4$ string theories," hep-th/0012118.

[97] E. Gava, A. B. Hammou, J. F. Morales and K. S. Narain, "AdS/CFT correspondence and D1/D5 systems in theories with 16 supercharges," hep-th/0102043.

[98] N. Hitchin, "Lectures on special Lagrangian submanifolds," math.DG/9907034.

[99] R. Bott and L. W. Tu, Differential forms in algebraic topology, SpringerVerlag, New York, 1982.

[100] E. Witten, "Duality relations among topological effects in string theory," JHEP0005, 031 (2000) [hep-th/9912086].

[101] J. A. Minahan, D. Nemeschansky, C. Vafa and N. P. Warner, "Estrings and $\mathrm{N}=4$ topological Yang-Mills theories," Nucl. Phys. B 527, 581 (1998) [hep-th/9802168]. 
[102] R. Dijkgraaf, E. Verlinde and H. Verlinde, "Matrix string theory," Nucl. Phys. B 500, 43 (1997) [hep-th/9703030].

[103] M. Bershadsky, T. Pantev and V. Sadov, "F-theory with quantized fluxes," Adv. Theor. Math. Phys. 3, 727 (1999) [hep-th/9805056].

[104] P. Berglund, A. Klemm, P. Mayr and S. Theisen, "On type IIB vacua with varying coupling constant," Nucl. Phys. B558, 178 (1999) [hepth/9805189].

[105] J. W. Milnor and J. D. Stasheff, Characteristic Classes (Princeton University Press, 1974).

[106] D. J. S. Robinson, A Course in the Theory of Groups, Graduate Texts in Mathematics 80, Springer, New York, 1982.

[107] C. B. Thomas, Characteristic Classes and the Cohomology of Finite Groups, Cambridge Studies in Advanced Mathematics 9, Cambridge University Press, Cambridge, 1986. 